


\title{
A CATALOGUE OF THE DIPTERA OF THE AMERICAS SOUTH OF THE UNITED STATES
}

\section{6}

\section{FAMILY SYRPHIDAE}

\author{
F. Christian Thompson
}

The American Museum of Natural History, New York, New York, U.S.A.

\section{J. R. VOCKEROTH}

Entomology Research Institute, Canada Department of Agriculture, Ottawa, Canada

YALE S. SEDman

Department of Biological Sciences, Western Illinois University Macomb, Illinois, U.S.A.

Adult Syrphidae, commonly called "Flower or Hover Flies", are rather conspicuous flies. Their size ranges from $4 \mathrm{~mm}$ to over $25 \mathrm{~mm}$ and their coloration from bright yellows and oranges to dull drab blacks and grays with a few iridescent forms. Many syrphid flies are Batesian mimics of stinging Hymenoptera and are highly beneficial as pollinators. The larvae of most syrphids are of little direct importance to man. A few are pests of Narcissus bulbs (Eumerus and Merodon), others have occasionally been reported to cause intestinal myiasis in man (Eristalis), and some of the predaceous syrphines are of importance in the control of aphids and other Homoptera.

Syrphid flies are wordwide in distribution, with their greatest diversity of forms in the New World tropics. Despite this interesting diversity and the critical zoogeographic importance of the Neotropical area, the South American forms are still poorly known. The higher classification of the Neotropical Syrphidae has been recently revised in large part by Vockeroth (1969, tribes of Syrphinae, genera of Syrphini) and Thompson (1969, Microdontinae; 1972, Milesiinae), but much still needs to be done on the specific level.

The first catalog of Neotropical Syrphidae was written by Williston (1886; corrections, 1887a). Aldrich's Catalogue of North American Diptera (1905) included many Neotropical species, since his coverage included Central America. Kertész (1910) provided a World catalog but his work on the New World was largely based on Williston and Aldrich. 
Some local catalogs were published (Brèthes, 1907 - Argentina; Stuardo, 1946 - Chile), but Fluke (1956-57) provided the first modern catalog to the Neotropical Syrphidae. However, as is usual with any such first attempts, there were numerous errors of both omission and commission in it. Goot (1946) reviewed Fluke's catalog, corrected some of his omissions, but still missed about a dozen species and caused additional confusion by introducting a few unnecessary names.

The present catalog is hopefully an improvement over Fluke's work but the large part of the credit for this improvement is due to his pioneering work and Goot's corrections. Besides the corrections of earlier errors, the other improvements in the present catalog stem from the use of a new higher classification of the family, as mentioned above. As a result our work, which tends to recognize distinct genera for the Neotropical species rather than to include them in worldwide genera as did earlier workers, contains many new combinations.

This catalog contains about 1,000 new combinations and synonyms. Most of these new changes have been indicated but undoubtedly some have escaped our notice. Many of the synonymies and combinations involving species of the earlier workers are based on the work of Vockeroth, who studied types of their species during trips to Europe in 1969 and 1971. Some of the other changes resulted from a short visit to the British Museum (Natural History) by Thompson and the loan of selected types by various curators to him. For all the gracious cooperation we received during our studies, we are very grateful to those curators listed below by their respective museums.

An attempt is made to list the depository of all the types of Neotropical species. These statements about type depositories start with an indication of the number of types, followed by the sex of the type or types and end with an abbreviation for the depository. The singular, type, is used when the species was described from a single specimen or a holotype or lectotype was or has been designated [in the case of lectotypes, reference to the designation is given]; the plural, types, when the species was described from a series and no lectotype has been designated; and "type(s)" when we are uncertain of the number of types incolved. The sex of the type or types is indicated by a sex symbol or query when the sex is unknown. The types are assumed to be where they were reported to be deposited either in the original publication or in other references (such as Horn \& Kahle, 1935-37; Sachtleben, 1961; Byers et al., 1962; and Zimsen, 1964). In cases of doubt about the depository of a type a quesy is added after the abbreviation for depository and when the depository is unknown only a query is used. All types seen by the authors are marked with an asterisk after the depository abbreviation. In a few cases where the type is missing, lost or destroyed this information is included in parentheses after the abbreviation and with either a reference to the source of the information or initials, which indicates which one of us has noted it.

AMNH The American Museum of Natural History, New York (P. Wygodzinsky*)

ANSP Academy of Natural Sciences, Philadelphia (D. Rentz*)

$\mathrm{BM}(\mathrm{NH}) \quad$ British Museum (Natural History), London (K.G.V. Smith*) CAS California Academy of Sciences, San Francisco (P. H. Arnaud, Jr.*)

CM Carnegie Museum, Pittsburg (G. Wallace*) 
CNC

CNHM

$\mathrm{CU}$

DEI

ENA

Hull

HZM

IML

IOC

Kiel

LSL

MA

MACN

MC

MCSNG

MCSNM

$\mathrm{MCZ}$

MD

MIZUN

ML

MNHN

MNHNS

MRHNB

MUNP

MZM

MZUF

MZUSP

NMB

NMPP

NRS

OhSU

OXF

Seabra

SMF

Thompson

UCD

UCSC

UCVC

Canadian National Collection, Ottawa (J. R. Vockeroth) Chicago National History Museum (Field Museum...), Chicago

Cornell University, Ithaca (L. L. Pechuman*)

Deutsches Entomologisches Institut, Eberswalde

Escola Nacional de Agronomia, Rio de Janeiro

Collection of F. M. Hull (now in the CNC).

Zoologisches Staatsinstitut und Zoologisches Museum, Hamburg

Instituto Miguel Lillo, Tucumán

Instituto Oswaldo Cruz, Rio de Janeiro (H. S. Lopes)

Fabricius Collection of Kiel Museum, now housed at MC

Linnean Society of London, Linnean Collection, now housed at $\mathrm{BM}(\mathrm{NH})$

Zoölogisch Museum, Amsterdam (W. Ellis*)

Museo Argentino de Ciencias Naturales "Bernardino Rivadavia", Buenos Aires

Universitetets Zoologiske Museet, Copenhagen (L. Lyneborg*)

Museu Civico di Storia Naturale "Giacomo Doria", Genova (D. Guiglia*)

Museo Civico di Storia Naturale, Milan (C. Leonardi*)

Museum of Comparative Zoology, Cambridge (H. E. Evans*)

Staatliches Museum für Tierkunde, Dresden

Museo dell' Istituto di Zoologia dell' Università di Napoli, Naples

Rijksmuseum van Natuurlijke Historie, Leiden (P. J. van Helsdingen*)

Muséum National d'Histoire Naturelle, Paris (L. Tsacas*)

Museo Nacional de Historia Natural, Santiago (J. Moroni B.*)

Musée Royal d'Histoire Naturelle de Belgique, Brussels

Museo, Universidad Nacional de La Plata, La Plata

Museum of Zoology, University of Michigan, Ann Arbor

Museo Zoologico dell'Università di Firenze, Florence (S. Carfi*)

Museu de Zoologia da Universidade de São Paulo (formely Departamento de Zoologia, Secretaria da Agricultura) (N. Papavero*)

Naturhistorisches Museum, Basel (F. Keiser*)

Národni Muzeum v Praze - Prírodovéddecké Muzeum, Prague (J. Jezék*)

Naturhistoriska Riksmuseet, Stockholm (P. I. Persson*)

Ohio State University, Columbus

Hope Department of Entomology, Oxford University, Oxford (D. M. Ackland*)

Collection of C. A. Campos Seabra, Rio de Janeiro

Senckenbergisches Museum, Frankfurt (W. Tobias*)

Collection of F. C. Thompson

Uuniversity of California, Davis

Centro de Estudios Entomológicos, Universidad de Chile, Santiago

Instituto de Biologia, Departamento de Ciencias, Universidad de Chile, Valparaiso 
UK

Snow Entomological Museum, University of Kansas, Lawrence (G. Byers*)

UNMB Természettudományi Múzeum, Budapest

USNM United States National Museum, Washington (L. Knutson, USDA, Systematic Entomology Laboratory*)

UTOR Istituto e Museo di Zoologia di Torino, Turin

UZMH Universitetets Zoologiska Museum, Helsinki (W. Hackman*)

VMNH Naturhistorisches Museum, Vienna (A. Kaltenbach*, R. Lichtenberg*)

ZMB Institut für Spezielle Zoologie und Zoologisches Museum der Humboldt-Universität zu Berlin, Berlin

ZML Zoological Museum, Lund (H. Andersson*)

ZSM Zoologische Staatssammlung, Munich

Geographical distribution is compiled only from Fluke's catalog (1956-57) and material seen by us. Other published distribution records not in Fluke or verified by us are not included and where we question the distribution records in Fluke, we have tried to indicate the source of the record. We have attempted to list the states or provinces of the various contries when specific locality data was available except only when the northern and southernmost Chilean province is given.

The arrangement of genera in the various subfamilies and tribes is shown, with an indication of the number of species, and a summary of the total number of species included in the major divisions of the family.

SYRPHINAE

Syrphini

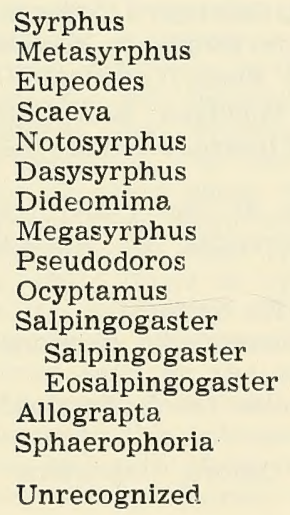

Chrysotoxini

Chrysotoxum

Paragini

Paragus 
Carposcalis

Melanostoma

Rhysops

Xanthandrus

Talaha.

Tuberculanostoma

4

Leucopodella

Unrecognized

Toxomerini

(143)

Toxomerus

Paragodon 2

Paramicrodon

Argentinomyia

Argentinomyia

Eurypterosyrphus

Mixogaster

Microdon

Ceratophya

Rhopalosyrphus

Rhoga

Ceriomicrodon

Masarygus

Ubristes

Microdon

Nothomicrodon

\section{MILESIINAE}

(774)

Pipizini

Pipiza

Trichopsomyia

Cheilosiini

Cheilosia

Cheilosia

Cartosyrphus

Rhingia

Chamaesyrphus

Volucellini

Ornidia

Tachinosyrphus

Copestylum

Callicerini 
Callicera

Notiocheilosia

Sericomyiini

Arctophila

Chrysogasterini

Lepidomyia

Myolepta

Myolepta

Protolepidostola

(6)

Chromocheilosia

3

Orthonevra

10

Cacoceria

Chamaesphegina

Eumerini

\section{Eumerus \\ Alipumilio \\ Nausigaster \\ Cerioidini \\ Sphiximorpha \\ Monoceromyia \\ Polybiomyia}

Unplaced

Eristalini

Helophilus

Polydontomyia

Asemosyrphus

Dolichogyna

Dolichogyna

Nosodepus

Quichuana

Mallota

Habromyia

Eristalis

Eristalis

Eoseristalis

Lycastrirhyncha

Palpada

Meromacrus

Milesiini

Xylota

Neplas

Ceriogaster 
$\begin{array}{lc}\text { Macrometopia } & 1 \\ \text { Sterphus } & 11 \\ \quad \text { Sterphus } & (2) \\ \text { Crepidomyia } & (9) \\ \text { Odyneromyia } & 2 \\ \text { Valdiviomyia } & 6 \\ \text { Aneriophora } & 1 \\ \text { Flukea } & 1 \\ \text { Criorhina } & 3 \\ \text { Philippimyia } & 1 \\ \text { Blera } & 1 \\ \text { Syritta } & 2 \\ \text { Senogaster } & 1 \\ \text { Tropidea } & 6 \\ \text { Milesia } & 5 \\ \text { Spilomyia } & 6 \\ \text { Stilbosoma } & 2 \\ \text { Hemixylota } & 3 \\ \text { unplaced species } & \\ \text { Unrecognized syrphids } & 1 \\ & \end{array}$

The initial manscript and literature survey for this catalog was completed by 15 May 1972, although some later references have been included in part. The last Zoological Record checked was 1968.

New names proposed in this catalog

Valdiviomyia Vockeroth

Ocyptamus isthmus Thompson

Ocyptamus octomaculatus Thompson

Ocyptamus willistoni Thompson

Allograpta rhina Thompson

Toxomerus hulli Sedman

Microdon anax Thompson

Copestylum colombiense Thompson

Copestylum dichopticum Thompson

Copestylum neosplendens Thompson

Copestylum neotropicum Thompson

Copestylum rufitarse Thompson

Copestylum shannoni Thompson

Copestylum trituberculatum Thompson

Copestylm vittatum Thompson

Quichuana nigricans Thompson

Palpada geniculosa Thompson

Palpada rufipedes Thompson

Ceriogaster hinei Thompson 


\title{
Subfamily SYRPHINAE
}

\author{
Tribe SYRPHINI
}

\author{
Ref. - Vockeroth, 1969 (generic rev.).
}

\section{Genus Syrphus Fabricius}

SYRPHUS Fabricius, 1775:762. Type-species, Musca ribesii Linnaeus (see Wirth et al. 1965:558) to preserve established usage. Ref. Fluke, 1942 (rev.).

octomaculatus Walker, 1837:344. Type-locality: “Chile". Distr. - Chile (Curicó-Magallanes), Argentina. Type क $\mathrm{BM}(\mathrm{NH}) *$. Ref. Fluke, 1942:3, Fig. 1 (abdomen).

gayi Macquart, 1842:150 (90), pl. 13, fig. 8 (head). Type-locality: "Chile". Type \& MNHN*.

testaceicornis Macquart, 1850:457 (153), pl. 14, fig. 12 (head). Typelocality: "Chile". Type \& MNHN* N. SYN.

patagonus Lynch Arribálzaga, 1892a:115 (1892c:73). Type-locality: Argentina, Patagonia, Río Gallegos. Type(s) ? MACN.

rALLIPES Bigot, 1884b:93. Type-locality: "Chile". Distr. - Chile, Argentina. Types o OXF*.

flavipes Shannon \& Aubertin, 1933:127, fig. 22a (abdomen), 22b (abdomen) (Scaeva). Type-locality: "Chile". Types o OXF* N. SYN.

PhaEostigma Wiedemann, 1830:130. Type-locality: "Brazil". Distr. (Santa Catarina, Rio de Janeiro), Argentina (Tucumán). Types o VMNH* Ref. - Fluke, 1942:2 (redescription), fig. 3 (abdomen); Fluke, 1950a:117, fig. 15 (male genitalia).

Poecilogaster Philippi, 1865:746. Type-locality: Chile, Valparaíso. Distr. Chile (Malleco-Magallanes). Type(s) ? MNHNS.

REEDI Shannon, 1927a:27. Type-locality: Chile, Valparaíso. Distr. - Chile, Brazil, Argentina. Type o USNM*.

similis Blanchard, 1852:410 (preocc. Fallén, 1817). Type-locality: Chile, Santa Rosa. Type o MNHM*.

pseudoribesii Vimmer \& Soukup, 1938:30. Type-locality: Peru, Puno. Type o NMPP* N. SYN.

SHORAE Fluke, 1950b:440 (nom. nov. for willistoni Fluke). Distr. - Mexico, Guatemala, Honduras, Costa Rica, Panama, Colombia, Ecuador, Chile, Argentina. Refs. - Fluke 1942:22, fig. 2 (abdomen); Fluke, 1950a:117, fig. 14 (male genitalia).

bisinuatus Williston, 1891:17 (preocc. Palma, 1863). Type-locality: Mexico, Guerrero, Omiltemi, 8,000 ft.; Costa Rica, Volcan de Irazu, 6,000-7,000 ft., Río Sucio. Types of $\mathrm{BM}(\mathrm{NH}) *$ \& $\mathrm{AMNH}^{*}$. willistoni Fluke, 1942:3 (nom. nov. for bisinuatus Williston; but preocc. Cockerell, 1909).

VITRIPENNIS Meigen, 1822:308. Type-locality: "Europe". Distr. - Holarctic; ?Mexico, Mexico City (Fluke, 1942:3). Type(s) ? MNHN Ref. - Fluke, 1950a:117, fig. 11 (male genitalia).

ribesii Linnaeus of Williston, 1891:17 and Giglio-Tos, 1893:27. 


\section{Genus Metasyrphus Matsumura}

METASyRPHUS Matsumura, in Matsumura \& Adachí, 1917:147. Type-species, Syrphus corollae Fabricius (orig. des.).

Posthosyrphus Enderlein, 1938:204. Type-species, Syrphus americanus Wiedemann (orig. des.). Ref. - Fluke, 1952 (rev.).

AMERICANUS (Wiedemann), 1830:129 (Syrphus). Type-locality: "North America". Distr. - North America; Middle America, Colombia. Type \& VMNH (not found-JRV). Ref. - Fluke, 1950a:132, figs. 184-186 (male genitalia).

wiedemanni Johnson, 1919:32 (Syrphus; unjunstified nom. nov. for americanus Wiedemann).

var. FlaviPes (Enderlein), 1938:205 (Posthosyrphus). Type-locality: Colombia, Bcgotá. Type o ZMB.

\section{Genus EupEodes Osten Sacken}

EuPEODES Osten Sacken, 1877:328. Type-species, volucris Osten Sacken (mon).

Fosthonia Enderlein, 1938:203. Type-species, longipenis Enderlein (orig. des.).

voluCRIS Osten Sacken, 1877:329. Type-locality: U.S.A., California, Los Angeles, Marin County, Yosemite; Nevada; Utah; Colorado, Denver, Clear Creek, Spanish Peaks. Distr. - North America; Mexico

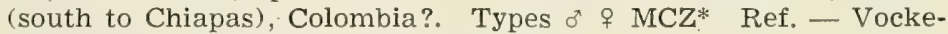
roth, 1969:150, fig. 34 (male genitalia).

?longipenis Enderlein, 1938:203 (Posthonia). Type-locality: Colombia, Bogotá. Types ơ of ZMB.

\section{Genus Scaeva Fabricius}

SCAEva Fabricius, 1805:248. Type-species, Musca pyrastri Linnaeus (Curtis, 1834: pl. 509). Ref. - Shannon \& Aubertin, 1933 (key).

MELANOSTOMA (Macquart), 1842:147 (87) (Syrphus). Type-locality: "Chile". Distr. - Ecuador (Azuay), Peru (Puno), Chile (south to Magallanes), Argentina (Tucumán, Santa Cruz), Type ? MNHN*. Ref. - Shannon, 1927:28 (redescription); Shannon \& Aubertin, 1933:130, fig. 23b (abdomen).

latafascies Macquart, 1850:456 (152) (Syrphus). Type-locality: "Chile". Types o $\sigma^{2}$ i $\mathrm{MNHN}$ *.

latifacies, Fluke, 1956:213, misspelled.

sexguttata Wulp, 1882:135 (Syrphus). Type-locality: "Argentina". Type o + ML?

simillima Vimmer \& Soukup, 1938:32 (Syrphus). Type-locality: Peru, Puno. Type \& NMPP* N. SYN.

pyrastri Linnaeus of Lynch Arribálzaga, 1892:121 (1892c:78), misident. occidentalis Shannon, 1927a:29. Type-locality: Chile, Santiago. Distr. Chile (Santiago-Aysén), Argentina (Tucumán, La Rioja, Mendoza, Neuquén, Río Negro). Type \& USNM* Ref. — Vockeroth, 1969: 150, fig. 36 (male genitalia).

sexmaculata Macquart, 1850:457 (153) (Syrphus; preocc. Palisot de Beauvois, 1819). Type-locality: Chile. Type o MNHN* N. SYN. chilensis Goot, 1964:214 (Syrphus; unjustified nom. nov. for sexmaculata Macquart). N. SYN. 
PUNCTATA Shannon \& Aubertin, 1933:128, fig. 23a (abdomen). Type-locality: Argentina, Bariloche. Distr. - Argentina (Tucumán, Neuquén). Type o* USNM*.

\section{Genus NotosyrpHus Vockroth}

NoTOSYRPHUS Vockeroth, 1969:72. Type-species, Metasyrphus golbachi Fluke (orig. des.).

GOLBACHI (Fluke), 1950b:447, fig. 9 (abdomen), 10 (head) \& 11 (male genitalia) [Metasyrphus (Posthosyrphus)]. Type-locality: Argentina, Tucumán, Villa Padre Monti. Distr. - Brazil (São Paulo, Santa Catarina, Paraná), Argentina (Tucumán, La Rioja). Type o IML. Ref. - Vockeroth, 1969:151, fig. 38 (male genitalia).

\section{Genus DASYSYRPHUS Enderlein}

DASYSYRPHUS Enderlein, 1938:208. Type-species, Scaeva albostriata Fallén (orig. des.).

LoTUS (Williston), 1887:75 (Syrphus). Type-locality: U.S.A., Arizona. Distr. - Western U.S.A.; Mexico (Mexico City), Ecuador? (Fluke, 1942:3). Type \& USNM.

\section{Genus Dideomima Vockeroth}

DIDEOMIMA Vockeroth, 1969:107. Type-species, Didea coquilletti Williston (orig. des.).

COQUILlETTI (Williston), 1891:19, pl. 1:9 (habitus), 9a (head) (Didea). Type-locality: Mexico, Guerrero, Omiltemi, 8,000 ft. Distr. Mexico (Durango, Jalisco, Guerrero, Chiapas). Types $\sigma^{\circ}+\mathrm{BM}$ $(\mathrm{NH}) *$ \& $\mathrm{AMNH}^{*}$. Refs. - Hull, 1925c:280, fig. 2 (abdomen), 6 (wing); Vockeroth, 1969:147, fig. 20 (wing tip), 161, fig. 67 (male genitalia).

\section{Genus Megasyrphus Dusek \& Láska}

MEgaSyrPHus Dusek \& Láska, 1967:363. Type-species, Scaeva annulipes Zetterstedt (orig. des.).

Laxus (Osten Sacken), 1875:66 (Didea). Type-locality: U.S.A., Michigan, Lake Superior; Maine, Norway; New Hampshire, Mt. Washington, Alpine region. Distr. - Alaska to Newfoundland, south to U.S.A. (California \& North Carolina), Mexico. Types of i MCZ*. Ref. Fluke, 1950a:126, figs. 111, 112 (male genitalia).

\section{Genus Pseudodoros Becker}

FSEUdodoros Becker, 1903:92. Type-species, nigricollis Becker (mon.). Eaccha, subg. Dioprosopa Hull, 1949e:99 (also, 1949d:296). Type-species, Syrphus clavatus Fabricius (mon.) N. SYN.

CLAVATUS (Fabricius), 1794:298 (Syrphus). Type-locality: "Americae meridionalis Insulis". Distr. - U.S.A. (California to Wisconsin and 
New Jersey) south to Brazil. Types o $q$ MC*. Ref. - Hull, 1949e:192, fig. 7 (abdomen); Hull, 1949d:288, fig. 9a (head). N. COMB.

scutellaris Walker, 1837:342 (Paragus?). Type-locality: "Brazil, St. Paul". Type ? BM(NH).

varia Walker, 1849:548 (Baccha). Type-locality: ? Types ? BM (NH).

facialis Thomson, 1869:504 (Baccha). Type-locality: Galápagos Islands. Types \& NRS*.

scutellata Williston, 1886:322 (?Mixogaster), misspelling.

\section{Genus Ocyptamus Macquart}

CCYPTAmus Macquart, 1834:554. Type-species, fascipennis Macquart (Coquillett, 1910a:577) = fuscipennis Say.

Baccha, authors.

Calostigma Shannon, 1927a:8. Type-species, elnora Shannon (orig. des.) N. SYN.

Callostigma, Curran, 1930f:8, 1930e:15, 1941:257, misspelled.

Callistigma, Hull, 1944j:41, misspelled.

Baccha, subg. Pelecinobaccha Shannon, 1927a:10. Type-species, peruviana Shannon (orig. des.). N. SYN.

Pipunculosyrphus Hull, 1937:29. Type-species, globiceps Hull (orig. des.). N. SYN.

Baccha, subg. Styxia Hull, 1943j:46. Type-species, eblis Hull (orig. des.). N. SYN.

Baccha, subg. Mimocalla Hull, 1943j:46. Type-species, Baccha capitata Loew (orig. des.). N. SYN.

Buccha, subg. Therantha Hull, 1943j:47. Type-species, Baccha atypica Curran (orig. des.). N. SYN.

Callisyrphus Frey, 1946:154. Type-species, Syrphus rubricosus Wiedemann (orig. des.) (1st reviser, Fluke, 1956:199). N. SYN.

Calliscaeva Frey, 1946:171. Incorrect original spelling.

Crphnabaccha Hull, 1949e:93. Type-species, Baccha coerulea Williston (orig. des.). N. SYN.

Baccha, subg. Atylobaccha Hull, 1949e:94. Type-species, Baccha flukiella Curran (orig. des.). N. SYN.

Buccha, subg. Aulacibaccha Hull, 1949e:96. Type-species, Baccha titan Hull (orig. des.). = arx Fluke. N. SYN.

Ii ercurymyia Fluke, 1950a:140. Type-species, Syrphus caldus Walker (orig. des.). N. SYN.

Hermesomyia Vockeroth, 1969:121. Type-species, bacchiformis Vockeroth (orig. des.) = phobifer Hull. N. SYN.

Pseudoscaevo Vockeroth, 1969:123. Type-species, Syrphus diversifasciatus Knab (orig, des.). N. SYN.

Ref. - Curran, 1941 (key); Hull, 1949e (rev., key).

ABata (Curran), 1938:1 (Baccha). Type-locality: Brazil, São Paulo, Campos do Jordão. Distr. - Brazil (São Paulo, Santa Catarina). Type of AMNH (missing)* Ref. - Hull, 1949e:222, fig. 143 (abdomen); 254, fig. 282 (wing). N. COMB. 
ADA (Curran), 1941:278 (Baccha). Type-locality: Brazil, Santa Catarina, Nova Teutônia. Distr. - same. Type ơ AMNH*. Ref. - Hull, 1949e:135 (redescription), 194, fig. 20 (abdomen); 216, fig. 116 (abdomen). N. COMB.

ADSPERSUS (Fabricius); 1805:200 (Baccha). Type-locality: "America meridionali". Distr. - Mexico (Chiapas), Colombia, Guyana, Panama, Ecuador, Peru, Brazil, Bolivia. Type o MC*. Ref. - Hull, 1943j:65, fig. 2 (alula); 1949e:138 (redescription), 198; fig. 33 (abdomen). N. COMB.

punctata Shannon, 1927a:11 (Baccha). Type-locality Bolivia, Beni, San Antonio. Type $0^{*} \mathrm{USNM}^{*}$.

AFNEUS (Williston), 1891:37, pl. 1:10 (habitus), 10a (head) (Baccha). Type-locality: Mexico, Guerrero, Sierra de las Aguas Escondidas, $9500 \mathrm{ft}$. Distr. - same. Types o $\mathrm{BM}(\mathrm{NH})$. N. COMB.

AEOLUS (Hull), 1943j:69, fig. 40 (abdomen) (Baccha). Type-locality: Ecuador, Machai, Río Pastaza. Distr. - same. Type o AMNH*. Ref. - Hull, 1949e:198, fig. 40 (abdomen). N. COMB.

AEQUILINEATUS (Hull), 1945b:71 (Epistrophe). Type-locality: Cuba, Pico Turquino, $6000 \mathrm{ft} .$, Summit. Distr. - same. Type $\&$ MCZ* N. COMB.

AGILIS (Bigot), 1884b:92 (Syrphus). Type-locality "Mexico". Distr. - same. Type o OXF? N. COMB.

ALICIA (Curran), 1941:278 (Baccha). Type-locality: Brazil, Santa Catarina, Nova Teutônia. Distr. - Brazil (São Paulo, Santa Catarina). Type o AMNH*. Ref. - Hull, 1949e:140 (redescription), 214, fig. 112 (abdomen). N. COMB.

AMABILIS (Hull), 1943k:39 (Baccha). Type-locality Peru, Loreto, Iquitos. Distr. - same. Type o USNM. Ref. - Hull, 1949e:192, fig. 4 (abdomen); 276, fig. 367 (wing). N. COMB.

AMPLUS (Fluke), 1942:6, fig. 7 (abdomen) (Epistrophe). Type-locality: Brazil, Santa Catarina, Nova Teutônia. Distr. - Brazil (São Paulo, Santa Catarina), Paraguay, Argentina (Tucumán). Type o AMNH*. Ref. - Fluke, 1950a:125, fig. 98 (male genitalia); Vockeroth, 1969:164, fig. 76 (male genitalia). N. COMB.

ANERA (Curran), 1939d:5 (Baccha). Type-locality: Brazil, Rondônia, Rio Madeira, Porto Velho. Distr. - same. Type o AMNH*. Ref. Hull, 1949e:246, fig. 257 (abdomen) ; 266, fig. 332 (wing). N. COMB. ANNulatus (Curran), 1941:258 (Callostigma). Type-locality: Brazil, Santa Catarina, Nova Teutônia, Distr. - Brazil (Santa Catarina), Argentina (Tucumán). Type ô AMNH*. Ref. - Hull, 1949e:230, fig. 184 (abdomen); 260, fig. 310 (wing). N. COMB.

ANONA (Hull), 1943j:91, fig. 2 (alula), 8 (abdomen) (Baccha). Typelocality: Ecuador, Oriente, Puyo. Distr. - same. Type o $\mathrm{AMNH}^{*}$. Ref. - Hull, 1949e:192, fig. 8 (abdomen). N. COMB.

ANTHinoNe (Hull), 1949e:179 (Baccha). Type-locality: Peru, Pucallpa. Distr. - same. Type ơ Hull. N. COMB.

ANTHiPHATES (Walker), 1849:589 (Syrphus). Type-locality: "Jamaica". Distr. - same. Type o BM(NH). N. COMB.

Akabella (Hull), 1947a:400 (Baccha). Type-locality: Costa Rica, La Suiza. Distr. - same. Type \& USNM. Ref. - Hull, 1949e:222, fig. 144 (abdomen); 266, fig. 335 (wing). N. COMB. 
ARETHuSA (Hull), 1949e:181 (Baccha). Type-locality: Peru, Pucallpa. Distr. - same. Type \& Hull. N. COMB.

aligentinus (Curran), 1939d:6 (Baccha). Type-locality: "Argentina". Distr. - Brazil (São Paulo, Santa Catarina), Argentina. Type ơ AMNH*. Ref. - Hull, 1949e:248, fig. 265 (abdomen); 266, fig. 328 (wing). N. COMB.

ARIELA (Hull), 1944i:398 (Baccha). Type-locality: "Brazil". Distr. - same. Type o VMNH. Ref. - Hull, 1949e:142 (redescription), 200, fig. 49 (abdomen); 270 , fig. 349 (wing). N. COMB.

IRX (Fluke), 1936:60, fig. 2 (abdomen), 3 (wing) (Baccha). Type-locality: Brazil, "Cois Parma Cos". Distr. - Brazil (Rio de Janeiro, Santa Catarina). Type o AMNH*. Ref. - Hull, 1949e:248, figs. 259-261 (abdomens); 274, fig. 361 (wing). N. COMB.

titan Hull, 1947a:395 (Baccha). Type-locality: Brazil, Santa Catarina, Nova Teutônia. Type đ̛ AMNH*. N. SYN.

ASTER (Curran), 1941:278 (Baccha). Type-locality: Brazil, Santa Catarina, Nova Teutônia. Distr. - same. Type $\sigma^{*} \mathrm{AMNH}^{*}$. Ref. - Hull, 1949e:200, fig. 41 (abdomen); 232, fig. 187 (abdomen); 238, fig. 216 (abdomen). N. COMB.

ATTENuatus (Williston), 1891:35 (Baccha). Type-locality: Mexico, Guerrero, Amula, $6000 \mathrm{ft}$.; Omiltemi, $8000 \mathrm{ft}$. Distr. - same. Types o $q \mathrm{BM}(\mathrm{NH}) *$ Ref. - Hull, 1949e:252, fig. 277 (abdomen). N. COMB.

ATypicus (Curran), 1930a:10. (Baccha). Type-locality: Brazil, Mato Grosso, Chapada. Distr. - Mexico, Surinam, Guyana, Brazil. Type o AMNH*. Ref. - Hull, 1949e:204, fig. 68 (abdomen); Doesburg, 1966:69, fig. 58 (habitus). N. COMB.

AURORA (Hull), 1943:73 (Baccha). Type-locality: Paraguay, Villarica. Distr. - same. Type o AMNH*. Ref. - Hull, 1949e:208, fig. 79 (abdomen). N. COMB.

BANKSI (Hull), 1941g:150 (Baccha). Type-locality: Panama, Bella Vista. Distr. - same. Type or MCZ. Ref. - Hull, 1949e:252, fig. 280 (abdomen). N. COMB.

PASSLERI (Curran), 1939d:8 (Baccha). Type-locality: Peru, Middle Río Ucayali. Distr. - same. Type o AMNH*. Ref. - Hull, 1949e:238, fig. 219 (abdomen); 256, fig. 297 (wing). N. COMB.

BEATRICEA (Hull), 1942c:73 (Baccha). Type-locality: Brazil, São Paulo (Hull, 1949e:144). Distr. - Brazil (São Paulo, Santa Catarina). Type \& Hull. Ref. - Hull, 1949e:144 (redescription), 202, fig. 53 (abdomen o); 220, fig. 137 (abdomen o) ; 272, fig. 354 (wing o ); 284, fig. 391 (wing of). N. COMB.

BlvitTatus (Curran), 1941:276 (Baccha), Type-locality: Brazil, Santa Catarina, Nova Teutônia. Distr. - same. Type o AMNH*. Ref. Hull, 1949e:200, fig. 48 (abdomen); 278, fig. 372 (wing). N. COMB. BONARIENSIS (Brèthes), 1905:340 (Baccha). Type-locality: Argentina, Buenos Aires. Distr. - same. Type o MACN. N. COMB.

BRAZILIENSIS (Curran), 1939d:9 (Baccha). Type-locality: Brazil, Mato Grosso, Poxoréu, Coronel Ponce, Distr. - same. Type \& AMNH*. Ref. - Hull, 1949e:212, fig. 101 (abdomen); 282, fig. 387 (wing). N. COMB.

BREVIPENNIS (Schiner), 1868:341 (Baccha). Type-locality: "South America". Distr. - Peru. Types ơ क VMNH*. Ref. - Hull, 1949e: 204, fig. 64 (abdomen); 268, fig. 343 (wing). N. COMB. 
BROMLEYI (Curran), 1929:490 (Baccha). Type-locality: Cuba, Santiago, Las Vegas. Distr. - same. Type o AMNH*. Ref. - Hull, 1949e:218, fig. 123 (abdomen); 274, fig. 362 (wing). N. COMB. BRUNNIPENNIS (Hull), 1942c:73 (Baccha). Type-locality: Brazil, São Paulo. Distr. - same. Type o Hull. Ref. - Hull, 1949e:206, fig. 76 (abdomen). N. COMB.

CALDUS (Walker), 1852:232 (Syrphus). Type-locality: "Brazil". Distr. Brazil (São Paulo, Santa Catarina), Paraguay, Argentina (Tucumán). Type o BM(NH)*. Ref. - Vockeroth, 1969:163, fig. 75 (male genitalia). N. COMB.

biarcuatus Fluke, 1937:4, fig. 6 (abdomen) (Epistrophe). Type-locality: Brazil, Santa Catarina, Nova Teutônia. Type o AMNH*.

CAllA (Curran), 1941:268 (Baccha). Type-locality: Brazil, Santa Catarina, Nova Teutônia. Distr. - Brazil (Santa Catarina), Argentina (Tucumán). Type o AMNH*. N. COMB.

Callidus (Hine), 1914:335 (Baccha). Type-locality: Guatemala, Puerto Barrios. Distr. - same. Type ơhSU. Ref. - Hull, 1949e:250, fig. 270 (abdomen). N. COMB.

CApitatus (Loew), 1863:14 (1864b:116) (Baccha). Type-locality: "Cuba". Distr. - same. Type o MCZ. Ref. - Hull, 1949e:196, fig. 22 (abdomen); 238, fig. 223 (abdomen); 240, fig. 227 (abdomen); 266, fig. 330 (wing). N. COMB.

CArlota (Curran), 1929:491 (Baccha). Type-locality: Cuba, Trinidad Mts., Mina Carlota. Distr. - same. Type \& $\mathrm{AMNH}^{*}$. Ref. - Hull, 1549e:238, fig. 224 (abdomen); 268, fig. 340 (wing). N. COMB.

CECrops (Hull), 1958:429 (Baccha). Type-locality: Peru, Chanchamayo. Distr. - same. Type o Hull. N. COMB.

celia (Hull), 1949e:214, fig. 108 (abdomen) (Baccha), Type-locality: ? Distr. - ? Type(s) o ? Nomen nudum.

CEREBerus (Hull), 1943j:66 (Baccha). Type-locality: Ecuador, Chicocha. Distr. - same. Type o AMNH*. Ref. - Hull, 1949e:234, fig. 203 (abdomen), N. COMB.

CHAPADENSIS (Curran), 1930a:14 (Baccha). Type-locality: Brazil, Mato Grosso, Chapada. Distr. - same. Type of AMNH*. Ref. - Hull, 1949e:240, fig. 226 (abdomen); 256, fig. 290 (wing). N. COMB.

Clarapex (Wiedemann), 1830:124 (Syrphus). Type-locality: "Brazil". Distr. - Brazil (Mato Grosso, Santa Catarina), Argentina (Misiones, Tucumán, Salta). Type \& VMNH*. Ref. - Hull, 1949e: 194, fig. 14 (abdomen. N. COMB.

COBEoldia (Hull), 1958:431 (Baccha). Type-locality: Peru, Chanchamayo. Distr. - same. Type o Hull. N. COMB.

COCHENILlivorus (Guérin-Méneville), 1848a:lxxxi (1848b:350) (Baccha). Type-locality: "l'Antigua Guatemala". Distr. - same. Type ? MNHN*. N. COMB.

COERUleus (Williston), 1891:38 (Baccha). Type-locality: Mexico, Guerrero; Omiltemi, $8000 \mathrm{ft}$.; Amula, $6000 \mathrm{ft}$.; Xucumanatlán, $7000 \mathrm{ft.;}$ Sierra de las Aguas Escondidas, $9500 \mathrm{ft}$. Distr. - U.S.A. (''exas), Mexico (Durango, Sinaloa, Morelos, Guerrero, Chiapas). 'Гypes o क $\mathrm{BM}(\mathrm{NH}) *$ Ref. - Hull, 1949e:224, fig. 159 (abdornen), 272, fig. 355 (wing); Vockeroth, 1969:163, fig. 74 (male genitalia). N. COMB.

Colombianus (Curran), 1941:282 (Baccha). Type-locality: Colombia, Magdalena, Río Frio, Cerro Patrón. Distr. - same. Type o* AMNH*. 
Ref. - Hull, 1949e:214, fig. 109 (abdomen); 270, fig. 346 (wing). N. COMB.

CoNCINNUS (Williston), 1891:38 (Baccha). Type-locality: Mexico, Chilpancingo, $4600 \mathrm{ft}$; Guerrero, Venta de Zopilote, $2800 \mathrm{ft}$; Tabasco, Teapa. Distr. - same. Types of \& BM(NH). N. COMB.

CONFORMIS Loew, 1866b:38 (1872b:98). Type-locality: "Cuba". Distr. same. Type \& MCZ.

CoNfusus (Goot), 1964:213 (Baccha; nom. nov. for Baccha valdiviana Philippi). Distr. - Chile. N. COMB.

valdiviana Philippi, 1865:750 (Baccha; preocc. Philippi, 1865:748). Type-locality: Chile, Corral. Type ? MNHNS.

CoNJUNCTUS (Wiedemann), 1830:116 (Syrphus). Type-locality: "South America". Distr. - Brazil. Type ơ MC*. N. COMB.

CORA (Curran), 1941:281 (Baccha). Type-locality: Brazil, Mato Grosso, Chapada. Distr. - same. Type o $\mathrm{AMNH}^{*}$. Ref. - Hull, 1949e: 144 (redescription); 194, fig. 16 (abdomen); 278, fig. 374 (wing); 284, fig. 390 (wing). N. COMB.

CORdelia (Hull), 1949e:186 (Baccha). Type-locality: Peru, Pucallpa. Distr. - same. Type o Hull. N. COMB.

COREOPSIS (Hull), 1944j:41 (Callistigma). Type-locality: Peru, Putumayo Distr., El Encanto. Distr. - same. Type o CU. Ref. - Hull, 1949e:206, fig. 75 (abdomen). N. COMB.

costatus (Say), 1829:161 (Baccha). Type-locality: U.S.A., Indiana. Distr. - Canada (Ontario), to U.S.A. (New Hampshire, south to Lousiana); Cuba. Type o lost. Ref. - Hull, 1949e:147 (redescription); 224, fig. 152 (abdomen + ); 228, fig. 169 (abdomen 万) ; 280, fig. 376 (wing). N. COMB.

?CRASSUS (Walker), 1852:222 (Baccha). Type-locality: Brazil. Distr. same. Type o $\mathrm{BM}(\mathrm{NH})$. N. COMB.

CROCATUS (Austen), 1893:155, pl. 5:5 (habitus) (Baccha). Type-locality: Brazil, Region of Amazon. Distr. - Mexico, Panama, Brazil. Type o BM(NH). Ref. - Hull, 1949e:198, fig. 35 (abdomen); 202, fig. 57 (abdomen). N. COMB.

CROCEUS (Austen), 1893:157, p1. 5:6 (habitus) (Baccha). Type-locality: Brazil, Region of Amazon. Distr. - Panama, Brazil. Types o $\mathrm{BM}(\mathrm{NH})$. Ref. - Hull, 1949e:200, fig. 47 (abdomen $0^{\pi}$ ); 210, fig. 88 (abdomen of) N. COMB.

CRYPTICUS (Hull), 1942a:97 (Baccha). Type-locality: Paraguay, Villarica. Distr. - same. Type o MCZ*. Ref. - Hull, 1949e:149 (redescription); 216, fig. 121 (abdomen). N. COMB.

CUBANUS (Hull), 1949j:62, fig. 25 (abdomen) (also, 1944c:30) (Baccha). Type-locality: Cuba, Soledad. Distr. - U.S.A. (Georgia), south to Cuba. Type \& MCZ. Ref. - Hull, 1949e:196, fig. 25 (abdomen). N. COMB.

CUbensis (Macquart), 1850:465 (161) (Baccha). Type-locality: "Cuba". Distr. - same. Type o MNHN*.

Cultratus (Austen), 1893:151, pl. 5:8-9 (habitus) (Baccha). Type-locality: Brazil, Pará, Santarém. Distr. — Guatemala, Guyana, Brazi!. Types o BM(NH). Ref. - Hull, 1949e:210, fig. 91 (abdomen). N. COMB.

var. INCISUS (Hull), 1949e:128 (Baccha). Type-locality: ?? Distr. — ?? Type (s) ? ?

var. PICTUlus (Hull), 1949c:737 (Baccha) [also 1949e:242, fig. 235 (abdomen) ]. Type-locality: Guyana, Essequibo River, Moraballi Creek, Wallaba Forest. Distr. - same. Type of BM(NH). 
Cultrinus (Curran), 1939d:7 (Baccha). Type-locality: Panama, Canal Zone, Barro Colorado Island. Distr. - same. Type o AMNH*. Ref. - Hull, 1949e:210, fig. 92 (abdomen); 242, figs 236 \& 241 (abdomen). N. COMB

currani Hull, 1941b:167 (Baccha). Type-locality: Panama, Canas Zone, Barro Colorado Island. Type o Hull.

CYBBLE (Hull), 1947b:236 (Baccha). Type-locality: Paraguay, Villarica. Distr. - same. Type or USNM. Ref. - Hull, 1949e:150 (redescription), 216, fig. 117 (abdomen). N. COMB.

CyClops (Hull), 1947a:406 (Baccha). Type-locality: Colombia, Muzo. Distr. - same. Type ot Hull. Ref. - Hull, 1949e:234, fig. 196 (abdomen). N. COMB.

CYLINDRICUS (Fabricius), 1781:429 (Syrphus). Type-locality: “Americae meridionalis insulis". Distr. - Jamaica, Haiti, Puerto Rico, Virgin Islands. Type ? Kiel* (only wings). Ref. - Hull, 1949e:218, fig. 130 (abdomen); 252, fig. 279 (abdomen); 280, fig. 380 (wing). N. COMB.

CYMBELlina (Hull), 1944g:63 (Baccha). Type-locality: Ecuador, Santo Domingo. Distr. - same. Type o AMNH*. Ref. - Hull, 1949e: 218, fig. 128 (abdomen). N. COMB.

DANAIDA (Hull), 19431:74 (Baccha). Type-locality: Brazil, Santa Catarina, Nova Teutônia. Distr. - same. Type o $\mathrm{AMNH}^{*}$. Ref. - Hull, 1949e:218, fig. 122 (abdomen). N. COMB.

DEBASA (Curran), 1941:272 (Baccha). Type-locality: Brazil, Santa Catarina, Nova Teutônia. Distr. - Brazil (São Paulo, Santa Catarina). Type of AMNH*. Ref. - Hull, 1949e:218, fig. 129 (abdomen), 280, fig. 378 (wing). N. COMB.

LECEPTOR (Curran), 1930a:14 (Baccha). Type-locality: Virgin Islands, St. Croix Islands. Distr. - Puerto Rico; Virgin Islands. Type c AMNH*. Ref. - Hull, 1949e:234, fig. 197 (abdomen), 254, fig. 287 (wing). N. COMB.

DECIPIENS (Williston), 1891:18 (Syrphus). Type-locality: Mexico, Guerrero, Omiltemi, $8000 \mathrm{ft}$. Distr. - Mexico (Durango, Guerrero, Chiapas), Brazil. Types o $\mathrm{BM}(\mathrm{NH}) *$ \& $\mathrm{AMNH} *$ Ref. - Fluke, 1942:22, fig. 11 (abdomen); Vockeroth, 1969:164, fig. 77 (superior lobe). N. COMB.

LFLiCATissimus (Hull), 1943m:214 (Baccha). Type-locality: Brazil, Santa Catarina, Nova Teutônia. Distr. - Brazil (São Paulo, Santa Catarina). Type o AMNH*. Ref. - Hull, 1949e:202, fig. 52 (abdomen); 252, fig. 278 (abdomen). N. COMB.

DIANA (Hull), 1947a:408 (Baccha). Type-locality: Guyana, Georgetown. Distr. - same. Type o Hull. Ref, - Hull, 1949e:234, fig. 204 (abdomen). N. COMB.

DIFFusus (Curran), 1939d:4 (Baccha). Type-locality: Brazil, São Paulo. Distr. - same. Type o AMNH*. Ref. - Hull, 1949e:242, fig. '238 (abdomen); 256, fig. 295 (wing). N. COMB.

Dimidiatus (Fabricius), 1781:434 (Syrphus). Type-locality "Americae meridionalis insulis". Distr. - Mexico south to Brazil; Dominican Republic, Puerto Rico, Lesser Antilles. Type ? ? Ref. - Hull, 1949e:240, fig. 232 (abdomen). N. COMB.

divisa Walker, 1857:156 (Pipiza). Type-locality: Mexico, Vera Cruz. Type $+\mathrm{BM}(\mathrm{NH})$.

dolosa Walker, 1857:156 (Pipiza). Type-locality: Brazil?, "Valley of Amazon". Type o BM(NH). 
var. RUFIFACIES (Doesburg), 1966:71 (Baccha). Type-locality: Surinam, Paramaribo. Distr. - same. Type of Doesburg.

DISJUNCTUS (Sack), 1921:132, fig. 3 (head), fig. 4 (mesonotum), fig. 5 (larva), fig. 6 (puparium) (Doros). Type-locality: Paraguay, St. Trinidad. Distr. - same. Types of o DEI. N. COMB.

Diversifaciatus (Knab), 1914:151 (Syrphus). Type-locality: U.S.A., California, Walnut Creek. Distr. - Canada (British Columbia) to U.S.A. (Utah, south to California, Arizona); Mexico. Type o USNM*. Ref. - Vockeroth, 1969:164, fig. 83 (male genitalia). N. COMB.

var. MERIDIONALIS (Fluke), 1950b:446, fig, 7-8 (male genitalia) [Stenosyrphus (Episyrphus)]. Type-locality: Argentina, Tucumán, Villa Padre Monti. Distr. - Argentina (Tucumán, Salta). Type o IML.

DIVERSUS (Williston), 1891:16, pl. 1:6 (habitus), 6a (head) (Syrphus). Type-locality: Mexico, Guerrero; Omiltemi, $8000 \mathrm{ft}$, , Xucumanatlán, $7000 \mathrm{ft}$. Distr. - Mexico (Guerrero, Chiapas). Types o $q$ $\mathrm{BM}(\mathrm{NH}) *$. N. COMB.

DoLorosus (Hull), 1950c:225 (Baccha). Type-locality: Peru, Chanchamayo. Distr. - same. Type or Hull. N. COMB.

Dracula (Hull), 1943a:52 (Baccha). Type-locality: Honduras, Puerto Castilla. Distr. - same. Type o Hull. Ref. - Hull, 1949e:152 (redescription), 232, fig. 205 (abdomen). N. COMB.

DFUIDA (Hull), 1947a:398 (Baccha). Type-locality: Brazil, Santa Catarina, Nova Teutônia. Distr. - Peru, Brazil (Santa Catarina, Paraná). Type o AMNH*. Ref. - Hull, 1949e:248, fig. 258 (abdomen). N. COMB.

DIYOPE (Hull), 1958:427 (Baccha). Type-locality: Peru, Chanchamayo. Distr. - same. Type ơ Hull. N. COMB.

DEIDA (Hull), 1947b:238 (Baccha). Type-locality: Venezuela, Mt. Duida. Distr. - Venezuela, Argentina (Tucumán, Salta). Type o AMNH*. Ref. - Hull, 1949e:250, fig. 267 (abdomen); 268, fig. 341 (wing). N. COMB.

EBLIS (Hull), 1943j:65, fig. 85 (abdomen) (Styxia). Type-locality: Ecuador, Bolivar, Hda. Talahua. Distr. - same. Type o $\mathrm{AMNH}^{*}$. Ref. - Hull, 1949e:208, fig. 85 (abdomen). N. CONiB.

FLEGans (Giglio-Tos), 1892d:2 (Melanostoma). Type-locality: Mexico, Orizaba. Types ot $q$ UTOR*. Ref. - Giglio-Tos, 1893:fig. 21 (head). N. COMB.

EuNORA (Shannon), 1927a:9, fig. 5 (wing tip) (Calostigma). Type-locality: Bolivia, Beni, Rurrenabaque. Distr. - Ecuador, Peru (Cuzco), Bolivia. Type o USNM. Ref. - Hull, 1949e:232, fig. 193 (abdomen); 260, fig. 308 (wing). N. COMB.

EREBUS (Hull), 1943h:137 (Baccha). Type-locality: Brazil, Santa Catarina, Nova Teutônia. Distr. - same. Type o" AMNH*. Ref. - Hull, 1949e:240, fig. 228 (abdomen). N. COMB.

Euraticus (Williston), 1888:264 (Syrphus). Type-locality: Brazil, Mato Grosso, Chapada. Distr. - Peru, Brazil (Mato Grosso). 'Types 3 \& AMNH*. Ref. - Fluke, 1942:22, fig. 10 (head); Vockeroth. 1969:164, fig. 78 (superior lobe), N. COMB.

Emprovs (Hull), 1943k:40 (Baccha). Type-locality: Peru, Loreto, Iquitos. Distr. - same. Type of USNM. Ref. - Hull, 1949e:204, fig. 61 (abdomen); 276, fig. 365 (wing). N. COMB. 
EXIGUUS (Williston), 1888:267 (Baccha). Type-locality: Brazil, Mato Grosso, Chapada. Distr. - same. Types ơ 우 AMNH*. Ref. - Hull, 1949e:200, fig. 46 (abdomen); 230, fig. 186 (abdomen). N. COMB. FASciatus Roeder, 1885:342. Type-locality: Porto Rico. Distr. - same. Type $\sigma^{\pi}$ (Zool. Univ. Mus., Halle a. S.; Horn \& Kahle, 1936:228). FERVIDUS (Austen), 1893:158, pl. 5:11 (habitus) (Baccha). Type-locality: Brazil, Pará, Santarém, R. Tapajós. Distr. - same. Type $\sigma^{*}$ $\mathrm{BM}(\mathrm{NH})$. N. COMB.

FIAMETTA (Hull), 1943j:75, fig. 13 (abdomen) (Baccha). Type-locality: Panama, Canal Zone, Barro Colorado. Distr. - same. Type o Hull. Ref. - Hull, 1949e:153 (redescription), 194, fig. 13 (abdomen). N. COMB.

FILII (Doesburg), 1966:73, fig. 59 (habitus, head, abdomen) (Baccha). Type-locality: Surinam, Paramaribo, Marienburg. Distr. - same. Type $0^{*}$ Doesburg. N. COMB.

Filiolus (Shannon), 1927a:30 (Baccha). Type-locality: Chile, Valparaiso. Distr. - Chile (Valparaíso, Santiago). Type o USNM. Ref. Hull, 1949e:230, fig. 180 (abdomen). N. COMB.

FJlissimus (Hull), 1943j:79, fig. 42 (abdomen) (Baccha). Type-locality: Colombia, Muzo. Distr. - same. Type + Hull. Ref. - Hull, 1949e:200, fig. 42 (abdomen). N. COMB.

Flatus (Hull), 1940b:433 (Baccha). Type-locality: Brazil, Rio de Janeiro, Petrópolis. Distr. - same. Type o Hull. Ref. - Hull, 1949e:202, fig. 56 (abdomen). N. COMB.

lopesae Curran, 1941:269 (Baccha). Type-locality: Brazil, Guanabara, Rio de Janeiro, Tijuca. Type o AMNH*. N. SYN.

FlAVENS (Austen), 1893:153, pl. 5:10 (habitus). (Baccha). Type-locality: Brazil, Region of Amazon. Distr. - same. Type o BM(NH). N. COMB.

FLAVIPENNIS (Wiedemann), 1830:123 (Syrphus). Type-locality: "Brazil". Distr. - Colombia, Ecuador, Peru, Brazil (São Paulo, Mato Gros" so). Types o \& ZMB. Ref. - Hull, 1949e:204, fig. 65 (abdomen). N. COMB.

FLUKEI (Curran), 1941:284 (Salpingogaster). Type-locality: Ecuador, Tungurahua, Baños. Distr. - same. Type o AMNH. Ref. - Hull, 1949e:240, fig. 229 (abdomen). N. COMB.

phobia Hull, 1943c:51 [Baccha (Mimocalla)]. Type-locality: Ecuador, Tungurahua, Baños, Chaupi. Type $\sigma^{*} \mathrm{AMNH}^{*}$.

FLUKiella (Curran), 1941:275 (Baccha). Type-locality: Brazil, Santa Catarina, Nova Teutônia. Distr. - same. Type o AMNH*. Ref. Hull, 1949e:198, fig. 39 (abdomen). N. COMB.

FRAGMENTARIUS (Hull), 1947a:405 (Baccha). Type-locality: Mexico, Tapachula Mts. Distr. - same. Type o AMNH*. Ref. - Hull, 1949e:242, fig. 237 (abàomen); 274, fig. 259 (wing). N. COMB.

Fraternus Bigot, 1883b:324. Type-locality: "Mexico". Distr. - same. Type o $\mathrm{BM}(\mathrm{NH})$ ? N. COMB.

FUNeBris Macquart, 1834:554. Type-locality: "Erazil". Distr. - U.S.A. (Texas), south to Brazil (São Paulo, Santa Catarina). Type(s) o MNHN (lost?)*. Ref. - Hull, 1949e:276, figs. 160 \& 162 (abdomens); 278, fig. 370 (wing). N. COMB.

iridipennis Walker, 1837:345 (Syrphus). Type-locality: "South America". Type o $\mathrm{BM}(\mathrm{NH})$.

F'rscicosta Lynch Arribálzaga, 1891:255 (1892c:52). Type-locality: Argentina, Buenos Aires, Chacabuco; La Plata. Distr. - same. Types? MACN \& MUNP. 
FUISCIPENnis (Say), 1823:100 (Baccha). Type-locality: U.S.A., Pennsylvania. Distr. - Canada (Quebec), west to U.S.A. (Wisconsin and Colorado), south to Mexico (Vera Cruz); Guadaloupe, Cuba. Type ? lost. Ref. - Hull, 1949e:222, fig. 145 (abdomen); 226, fig. 167 (abdomen); 232, fig. 192 (abdomen); 280, fig. 379 (wing). N. COMB.

fenestratus Bigot, 1885a:251. Type-locality: "Mexico". Type o OXF. N. SYN.

fenestratus Hull, 1949e:280, fig. 381 (wing) (Baccha; as a variety of fuscipennis; preocc. Bigot, 1885). Nomen nudum.

GASTROSTACTUS (Wiedemann), 1830:123 (Syrphus). Type-locality: "Brazil". Distr. - U.S.A. (Texas \& Florida), south to Brazil (Santa Catarina). Types o VMNH* \& AMNH*. Ref. - Hull, 1943j:65, fig. 2 (alula); 1949e:226, figs. 163-164 (abdomens); 258, figs. 299-300 (wings). N. COMB.

trigonus Wiedemann, 1830:126 (Syrphus). Type-locality: "Brazil". Types $\sigma^{*} \mathrm{ZMB} \& \mathrm{VMNH}$ (missing)*.

costalis Walker, 1837:342 (Pipiza, preocc. Wiedemann, 1830). Typelocality: South America. Type o $\mathrm{BM}(\mathrm{NH})$.

pica Walker, 1857:156 (Pipiza). Type-locality: "Valley of Amazon". Type o $\mathrm{BM}(\mathrm{NH})$.

notatus Coquillett, 1902:195 (preocc. Loew, 1866). Type-locality: Mexico, Tabasco, Frontera. Types o USNM.

GEIJSKESI (Doesburg), 1966:75, fig. 60 (habitus) (Baccha). Type-locality: Surinam, Paramaribo, Botanical Garden (Cultuurtuin). Distr. same. Type $\sigma^{7}$ Doesburg. N. COMB.

Grganteus (Schiner), 1868:340 (Baccha). Type-locality: "South America". Distr. - same. Types o \& VMNH*. N. COMB.

GILvUS (Austen), 1893:154, pl. 5:7 (habitus) (Baccha). Type-locality: Brazil, Region of Amazon. Distr. - same, Type ơ $\mathrm{BM}(\mathrm{NH}) *$. N. COMB.

GLOBICEPS (Hull), 1937b:30 (Pipunculosyrphus). Type-locality: Paraguay, San Bernardino. Distr. - Brazil (Santa Catarina), Paraguay. Type o VMNH. Ref. - Hull 1949e:192, fig. 3 (abdomen); 266, fig. 334 (wing). N. COMB.

tiara Curran, 1941:271 (Baccha). Type-locality: Brazil, Santa Catarina, Nova Teutônia. Type \& AMNH*.

GoLBACHI (Fluke), 1950b:441, fig. 1 (abdomen) [Stenosyrphus (Episyrphus)]. Type-locality: Argentina, Tucumán, Villa Padre Monti. Distr. same. Type o IML. N. COMB.

Gratus (Curran), 1941:270 (Baccha). Type-locality: Brazil, Mato Grosso, Chapada. Distr. - same. Type o $\mathrm{AMNH}^{*}$. Ref. - Hull, 1949e: 236, fig. 214 (abdomen), 276, fig. 366 (wing). N. COMB.

fi.llcyone (Hull), 1949b:244 (Baccha). Type-locality: Peru, Chanchamayo, 1100 m. Distr. - same. Type o Hull. N. COMB.

IJARLEQUiNUS (Hull), 1948a:10 (Baccha). Type-locality: Brazil, Rio Grande do Norte, Natal. Distr. - Brazil (Natal), Argentina. Type $\sigma^{*}$ AMNH*. Ref. - Hull, 1949e:250, fig. 268 (abdomen), 254, tig. 289 (wing). N. COMB.

Hrantha (Hull), 1943j:74 (Baccha). Type-locality: Brazil, Santa Catarina, Nova Teutônia. Distr. - same. Type q AMNH*. Ref. - Hull, 1949 e:154 (redescription), 212, fig. 96 (abdomen), 272, fig. 356 (wing). N. COMB.

HIPPolyte (Hull), 1957:187 (Baccha). Type-locality: Peru, Chanchamayo. Distr. - same. Type o Hull. N. COMB. 
HiRTUS (Shannon), 1927a:11 (Baccha). Type-locality: Bolivia, Beni, Ivon. Distr. - same. Type or USNM, Ref. - Hull, 1949e:154 (redescription), 250, fig. 271 (abdomen). N. COMB.

HIRUNDELlA (Hull), 1944g:59 (Baccha). Type-locality: Brazil, Santa Catarina, Nova Teutônia, Distr. - same. Type o AMNH. Ref. Hull, 1949e:155 (redescription), 212, fig. 99 (abdomen). N. COMB. riYacinthia (Hull), 1947a:409 (Baccha). Type-locality: Cuba, Soledad. Distr. - same. Type \& Hull. Ref, - Hull, 1949e:246, fig. 252 (abdomen). N, COMB.

hraltPennis (Curran), 1930e:15 (Callostigma). Type-locality: Honduras, Tegucigalpa. Distr. - Honduras, Argentina (Tucumán). Type $\Varangle$ AMNH*. Ref. - Hull, 1949e:230, fig. 183 (abdomen), 260, fig. 311 (wing). N. COMB.

IDA (Curran), 1941:279 (Baccha). Type-locality: Brazil, Santa Catarina, Nova Teutônia. Distr. - Brazil (Santa Catarina), Paraguay, Argentina (Jujuy). Type o AMNH*. Ref. - Hull, 1949e:156 (redescription), 194, figs. 12, 18 (abdomen), 262, fig. 319 (wing), 274, fig. 358 (wing). N. COMB.

var. IDELla (Hull), 1949e:159, fig. 107 (abdomen) (Baccha). Typelocality: Paraguay, Villarica. Type o USNM.

IDANus (Curran), 1941:276 (Baccha). Type-locality: Peru, Rio Putumayo. Distr. - same. Type of AMNH*. Ref. - Hull, 1949e:240, fig. 230 (abdomen), 278, fig. 373 (wing). N. COMB.

IMMACULATUS (Macquart), 1842:158 (98), tab. 17, figs, 1 (head), 1a (wing) (Syrphus). Type-locality: "Chile or Brazil". Distr. - same.

Type o MNHN*. N. COMB.

INCA (Curran), 1939d:4 (Baccha). Type-locality: Peru. Distr. - same. Type o $\mathrm{AMNH}^{*}$. Ref. - Hull, 1949e:200, fig. 50 (abdomen). N. COMB.

INFANTA (Hull), 1943j:74 (Baccha). Type-locality: Colombia, Muzo. Distr. - same. Type o Hull, 1949e:159 (redescription), 224, fig. 151 (abdomen). N. COMB.

INFuscatus Bigot, 1883b:324. Type-locality: Mexico. Distr. - Mexico, Jamaica, Puerto Rico. Types o BM(NH). N. COMB.

INORNATUS (Walker), 1837:342 (Baccha). Type-locality: "South America". Distr. - same. Type o BM(NH). N. COMB.

INSUlaris (Bigot), 1883b:330 (Doros). Type-locality: "Cuba". Distr, same. Type of $\mathrm{BM}(\mathrm{NH})^{*}$ N. COMB.

In (Hull), 1944g:61 (Baccha). Type-locality: Brazil, São Paulo, Campos do Jordão. Distr. - same. Type o AMNH*, Ref. - Hull, 1949e: 236, fig. 208 (abdomen), 268, fig. 342 (wing). N. COMB.

IONA (Curran), 1941:265 (Baccha). Type-locality: Brazil, Mato Grosso, Chapada. Distr. - same. Type o AMNH*. Ref, - Hull, 1949e: 242, fig. 234 (abdomen), 278, fig. 371 (wing).

IRis Austen, 1893:133, pl. 4:1 (habitus). Type-locality: Jamaica, Cinchona. Distr. - same. Type \& $\mathrm{BM}(\mathrm{NH})$. N. COMB.

ISTHMUS Thompson (for Callostigma panamensis Curran). Distr. - Panama. Ref. - Hull, 1949e:212, fig. 103 (abdomen). NOM. NOV.

panamensis Curran, 1930f:8 (Callostigma; preocc. Curran, 1930). Typelocality: Panama, Canal Zone, Barro Colorado Island. Type $q$ $\mathrm{AMNH} *$.

JACTATOR (Loew), 1861:40 (Syrphus). Type-locality: Cuba. Distr. - U.S.A. (Florida), Cuba. Type o MCZ*. Ref. - Fluke, 1942:23, fig. 33 (abdomen); 1950a:127, fig. 133 (male genitalia). N. COMB.

JoHnsoni (Curran), 1934b:392 (Baccha). Type-locality: Guyana, Bartica. 
Distr. - same. Type \& MCZ. Ref. - Hull, 1949e:159 (redescription), 252, fig. 281 (abdomen). N. COMB.

LANEI (Fluke), 1950b:444, fig. 4 (male genitalia) [Stenosyrphus (Episyrphus) ]. Type-locality: Brazil, São Paulo, Campos do Jordão. Distr. - Brazil (São Paulo, Rio de Janeiro). Type o MZUSP. N. COMB.

LATICAUdA (Curran), 1941:2]7 (Baccha). Type-locality: Brazil, São Paulo. Distr. - same. Type o AMNH*. Ref. - Hull, 1949e:224, fig. 158 (abdomen), 266, fig. 333 (wing). N. COMB.

Latrusculus Loew, 1866b:39 (1872b:99). Type-locality: Cuba. Distr. U.S.A. (Florida); Cuba, Jamaica. Type o MCZ.

LATIVENTRIS (Curran), 1941:277 (Baccha). Type-locality: Brazil, Santa Catarina, Nova Teutônia. Distr. - same. Type of AMNH*. Ret. - Hull, 1949e:244, fig. 246 (abdomen). N. COMB.

LAUdABILIS (Williston), 1891:36 (Baccha). Type-locality: Mexico, Guerrero, Sierra de las Aguas Escondidas, $7000 \mathrm{ft}$. Distr. - same. Type $q$ $\operatorname{BM}(\mathrm{NH}) * \quad$ N. COMB.

LAUTUS (Giglio-Tos), 1892d:2 (Syrphus). Type-locality: Mexico, Tuxpango. Distr. - same. Type o UTOR*. Ref. - Giglio-Tos, 1893:figs. 4 (abdomen), 4a (head). N. COMB.

LEPIDUS (Macquart), 1842:169 (109), tab. 19, fig. 1 (habitus) (Baccha). Type-locality: ??? Distr. - Mexico, Panama. Type(s) o MNHN. Ref. - Hull, 1949e:192, fig. 5 (abdomen), 202, fig. 58 (abdomen). N. COMB.

Leucopodus (Hull), 1948a:5 (Baccha). Type-locality: Brazil, Goiás. Distr. - Brazil (Goiás, São Paulo). Type \& Hull. Ref. - Hull, 1949e: 160 (redescription), 212, fig. 100 (abdomen). N. COMB.

LEVISSIMUS (Austen), 1893:146, pl. 4:15 (habitus) (Baccha). Type-locality: Brazil, Region of Amazon. Distr. - Peru, Brazil. Types o $\mathrm{BM}(\mathrm{NH})$. Ref. - Hull, 1949e:238, fig. 220 (abdomen), 268, tig. 337 (wing). N. COMB.

LIMPIDAPEX (Curran), 1941:282 (Baccha). Type-locality: Brazil, Mato Grosso, Chapada. Distr. - same. Type o AMNH*. Ref. - Hull, 1949e:212, fig. 104 (abdomen), 282, fig. 385 (wing), N. СОМB.

LINEATUS (Macquart), 1846:267 (139), tab. 20, fig. 5 (habitus) (Baccha). Type-locality: Mexico, Yucatån. Distr. U.S.A. (Florida, 'l'exas), Mexico (Yucatán). Type o MNHN? Ref. - Hull, 1949e: 236, fig. 206 (abdomen). N. COMB.

var. connexus (Hull), 1949e:236, fig. 207 (abdomen) (Baccha). Typelocality: ? Type ? ? Nomen nudum.

LIvidus (Schiner), 1868:343 (Baccha). Type-locality: "South America". Distr. - Mexico, Honduras, Panama, Colombia, Guyana, Trinidad, Peru, Brazil. Types o \& VMNH*. Ref. - Hull, 1949e:196, fig. 26 (abdomen), 281, fig. 124 (abdomen), 268, fig. 336 (wing). N. COMB.

LitCretta (Hull), 1949i:230 (Baccha). Type-locality: Peru, Chanchamajo. Distr. - same. Type of Hull. N. COMB.

Luctuosus (Bigot), 1883b:334 (Baccha). Type-locality: "Mexico". Distr. Mexico, ?Costa Rica (Fluke, 1956:252). Type o o $^{\star} \mathrm{BM}(\mathrm{NH})$. Ref. Hull, 1949e:242, fig. 239 (abdomen). N. COMB.

LUGUBRIS (Philippi), 1865:749 (Baccha). Type-locality: Chile, Corral. Distr. - Chile (Aysén). Type(s) ? MNHNS. N. COMB.

nigrifrons Bigot, 1883b:335 (Baccha). Type-locality: Chile. Type o $\mathrm{BM}(\mathrm{NH})$. 
MACER (Curran), 1930a:13 (Baccha). Type-locality: Paraguay, San Bernadino. Distr. - same. Type o $\mathrm{AMNH}^{*}$. Ref. - Hull, 1949e:246, fig. 251, (abdomen), 260, fig. 309 (wing). N. COMB.

MACROPYGA (Curran), 1941:267 (Baccha). Type-locality: Brasil, Santa Catarina, Nova Teutônia. Distr. - same. Type $\sigma^{*} \mathrm{AMNH}$ *. Ref. Hull, 1949e:196, fig. 24 (abdomen), 244, figs, 242-243 (abdomens), 260, fig. 313 (wing). N. COMB.

MARA (Curran), 1941:274 (Bacch $\alpha$ ). Type-locality: Brazil, Santa Catarina, Nova Teutônia. Distr. - Brazil (Santa Catarina), Argentina (Tucumán). Type o AMNH*. Ref. - Hull, 1949e:246, fig. '253 (abdomen), 256, fig. 293 (wing).

MELANORRHiNus (Philippi), 1865:749 (Baccha). Type-locality: Chile, Valdivia. Distr. - Chile (Curicó-Aysén). Type? MNHNS. Ref. Hull, 1949e:224, fig. 155 (abdomen), 258, fig. 301 (wing). N. COMB.

felix Shannon, 1927a:30 (Baccha). Type-locality: Chile, Valparaiso. Type o USNM.

MENToR (Curran), 1930a:12 (Baccha). Type-locality: Paraguay, San Ber-

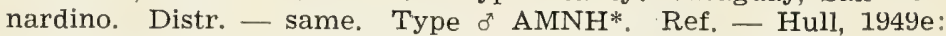
248, fig. 263 (abdomen), 254, fig. 286 (wing). N. COMB.

MEXICANUS (Curran), 1930a:6 (Baccha; nom. nov, for lugubris Williston). Distr. - Mexico (Vera Cruz, Guerrero, Tabasco). Ref. - Hull, 1949e:216, fig. 114 (abdomen); 274, fig. 360 (wing). N. СОМB.

lugubris Williston, 1891:37 (Baccha; preocc. Philippi, 1865). Typelocality: Mexico, Tabasco, Venta de Zopilote, $2800 \mathrm{ft}$., Teapa; Guerrero, Chilpancingo, $4600 \mathrm{ft}$, , Vera Cruz, Atoyac. Types $o^{\pi} q$ $\mathrm{BM}(\mathrm{NH})$ \& AMNH*.

batesi Curran, 1939d:10 (Baccha). Type-locality: Guatemala, San Sebastian, Reu. Type \& AMNH*. [See Hull, 1949e:220, fig. 136 (abdomen); 282, fig. 382 (wing) ].

MirCROPELECINUS (Shannon), 1927a:10 (Baccha). Type-locality: Bolivia Tumupasa. Distr. - same. Type o USNM. Ref. - Hull, 1949e. 250, fig. 269 (abdomen). N. COMB.

MICROPYGA (Curran), 1941:271 (Baccha). Type-locality: Brazil, Santa Catarina, Nova Teutônia. Distr. - same. Type o AMNH. N. COMB.

var. iolanthe Hull, 1949e:252, fig. 276 (abdomen) (Baccha). Locality: "Brazil". Type ? ? Nomen Nudum.

mimus (Hull), 1949e:188 (Baccha). Type-locality: Peru, Pucallpa. Distr. - same. Type \& Hull. N. COMB.

MrNImus (Hull), 1943m:214 (Baccha). Type-locality: Brazil, Santa Catarina, Nova Teutônia. Distr. - same. Type o AMNH*. Ref. Hull, 1949e:208, fig. 78 (abdomen). N. COMB.

mimima, Hull, 1949e:208, misspelled.

MURINUS (Curran), 1930a:12 (Baccha). Type-locality: Brazil, Mato Grosso, Chapada. Distr. - same. Type o AMNH*. Ref. - Hull, 1949e: 230, fig. 181 (abdomen); 268, fig. 338 (wing). N. COMB.

MYrtella (Hull), 1960:70 (Baccha). Type-locality: Peru, Chanchamayo. Distr. - same. Type o Hull. N. COMB.

NASUTUS (Williston), 1891:35 (Baccha). Type-locality: "Mexico". Distr. same. Types ơ \& BM(NH). Ref. - Giglio-Tos, 1893:fig. 8 (head). N. COMB.

Nectarinus (Hull), 1942a:94 (Baccha). Type-locality: Paraguay, Villarica. Distr. - same. Type or MCZ. Ref. - Hull, 1949e:196, fig. 30 (abdomen). N. COMB.

NEPTUNUS (Hull), 1943h:138 (Baccha). Type-locality: Brazil, Rio de Ja- 
neiro, Japuiba, Angra dos Reis. Distr. - same. Type o AMNH* . Ref. - Hull, 1949e:192, fig. 9 (abdomen). N. COMB.

NERISSA (Hull), 1943m:215 (Baccha). Type-locality: Ecuador, Pinas. Distr. - same. Type o AMNH*. Ref. - Hull, 1949e:162 (redescription), 212, fig. 97 (abdomen). N. COMB.

NERo (Curran), 1939d:8 (Baccha). Type-locality: Guyana, Kaieteur. Distr. - same. Type o BM(NH). N. COMB.

Nruralis (Curran), 1934b:395 (Callostigma). Type-locality: Guyana, Kartabo. Distr. - same. Type o AMNH*. Ref. - Hull, 1949e:232, fig. 195 (abdomen); 260, fig. 312 (wing), N. COMB.

Nigrocilia (Hull), 1943m:215 (Baccha). Type-locality: Brazil, São Paulo, Ilha Seca. Distr. - Brazil (São Paulo), Colombia. Type of AMNH*. Ref. - Hull, 1949e:162 (redescription), 244, fig. 248 (abdomen). N. COMB.

var. HIRTIPES (Hull), 1943m:215 (Baccha), Type-locality: "Colombia". Type ? AMNH (missing - FCT). Ref, - Hull, 1949e:220, fig. 139 (abdomen).

var. INCLUSA (Hull), 1943m:215 (Baccha). Type-locality: "Colombia". Type ? AMNH (missing - FCT). Ref, - Hull, 1949e:224, tig. 156 (abdomen).

NIOBE (Hull), 19431:73 (Baccha). Type-locality: Ecuador, Palmar, Manabj 200 m. Distr. - same. Type o AMNH*. Ref. - Hull, 1949e:IU8, fig. 37 (abdomen). N. COMB.

Nitidulus (Curran), 1930a:9 (Baccha). Type-locality: Panama, Canal Zone, Barro Colorado Island. Distr. - Panama, Brazil (Rondônia). Type o AMNH*, Ref. - Hull, 1949e:214, fig. 105 (abdomen). N. COMB.

Nodosus (Hull), 1930:141, fig. 8 (abdomen) (Baccha). Type-locality: Mexico, Vera Cruz, Jalapa. Distr. - same. Type o ANSP. Ref. Hull, 1949e:192, fig. 6 (abdomen). N. COMB.

NoRA (Curran), 1941:269 (Baccha). Type-locality: Brazil, Santa Catarina, Nova Teutônia. Distr. - same. Type o AMNH*. N. COMB.

NORINA (Curran), 1941:267 (Baccha). Type-locality: Brazil, Santa Catarina, Nova Teutônia. Distr. - Brazil (São Paulo, Santa Catarina), Bolivia, Argentina (Misiones, Formosa, Tucumán, Jujuy, Salta). Type o AMNH*. Ref. Hull, 1949e:196, fig. 23 (abdomen), 218, fig. 131 (abdomen), 270, fig. 347 (wing). N. COMB.

Notatus (Loew), 1866b:37 (1872b:97) (Baccha). Type-locality: "Cuba". Distr. - same. Type ot MCZ*. Ref. - Hull, 1949e:236, fig. 211 (abdomen), 240, fig. 233 (abdomen), 256, fig. 294 (wing). N. COMB. NYMPHAEA (Hull), 1943k:40 (Baccha). Type-locality: Brazil, São Paulo, Campinas, Distr. - same. Type o USNM. Ref. - Hull, 1949e: 238, fig. 221 (abdomen). N. COMB.

OßzlquUs (Curran), 1941:258 (Callostigma). Type-locality: Panama, Canal Zone, Chiva Chiva Trail. Distr. - same. Type \& AMNH*. Ref. - Hull, 1949e:230, fig. 185 (abdomen), 260, fig. 307 (wing). N. COMB.

oBLongus (Walker), 1852:221 (Baccha). Type-locality: Brazil. Distr, same. Type of $\mathrm{BM}(\mathrm{NH})$. N. COMB.

OBSOLETUS (Curran), 1941:266 (Baccha). Type-locality: Paraguay, San Bernadino. Distr. - same. Type o AMNH*. Ref. - Hull, 1949e: 222, fig. 150 (abdomen), 282, fig. 383 (wing). N, COMB.

oulireolinea (Hull), 1944c:31 (Baccha) [also, 1943j:98, fig. 89 (abdomen) ]. Type-locality: Panama, Canal Zone, Barro Colorado Island. Distr. 
— same. Type of MCZ*. Ref. - Hull, 1949e:210, fig. 89 (abdomen). N. COMB.

octomaculatus Thompson (nom. nov. for flukei Hull). Distr. - Brazil (Santa Catarina). Ref. - Hull, 1949e:198, fig. 36 (abdomen). NOM. NOV.

flukei Hull, 1943j:67, fig. 36 (abdomen) (Baccha; preocc. Curran, 1941). Type-locality: Brazil, Santa Catarina, Nova Teutônia. Type + AMNH* .

OENONE (Hull), 1949e:109, fig. 198 (abdomen) (Baccha). Type-locality: "Jamaica". Distr. - same. Type $\sigma^{*}$ ? N. COMB.

opacus (Fluke), 1950b:443, fig. 2 (abdomen), fig. 3 (male genitalia) [Stenosyrphus (Episyrphus)]. Type-locality: Ecuador, Tungurahua, Mt. Tungurahua, $2800 \mathrm{ft}$. Distr. - same. Type of $\mathrm{AMNH}^{*}$. Ref. - Vockeroth, 1969:164, fig. 80 (superior lobe). N. COMB.

ORIEL (Hull), 1942a:96 (Baccha). Type-locality: Dominican Republic, Loma Rucilla and Mts N., 5000-8000 ft. Distr. - same. Type o MCZ. Ref. - Hull, 1949e:196, fig. 29 (abdomen), 218, fig. 126 (abdomen). N. COMB.

ORNATIPES (Curran), 1927b:3 (Baccha). Type-locality: Puerto Rico, Cayey. Distr. - same. Type ơ AMNH*. Ref. - Hull, 1949e:232, fig. 194 (abdomen), 260, fig. 306 (wing). N. COMB.

ovipHORUS (Hull), 1943j:73 (Baccha). Type-locality: Colombia, Muzo. Distr. - same. Type of Hull. Ref. - Hull, 1949e:232, fig. 191 (abdomen). N. COMB.

CVIPOSITORIUS (Hull), 1943j:76 (Baccha), Type-locality: Colombia. Distr. - same. Type \& Hull. Ref. - Hull, 1949e:230, fig. 178 (abdomen). N. COMB.

PANAMENSIS (Curran), 1930a:9 (Baccha). Type-locality: Panama, Canal Zone, Barro Colorado Island. Distr. - same. Type of $\mathrm{AMNH}^{*}$. Ref. - Hull, 1949e:212, fig. 103 (abdomen). N. COMB.

PANDORA (Hull), 1942i:102 (Baccha). Type-locality: Panama, Coclé prov., El Valle. Distr. - same. Type o MCZ. Ref. - Hull, 1949e:198, fig. 38 (abdomen). N. COMB.

PAPILIO (Hull), 1942d:45 (Baocha). Type-locality: Brazil, São José (?). Distr. - Brazil (Santa Catarina). Ref. - Hull, 1949e:198, fig. 38 (abdomen). N. COMB.

PAPILIONARIUS (Hull), 1943j:71 (Baccha). Type-locality: Brazil, Santa Catarina, Nova Teutônia. Distr. - same. Type \& $\mathrm{AM}(\mathrm{NH}) *$. Ref. Hull, 1949e:220, fig. 133 (abdomen). N. COMB.

PARA (Curran), 1941:280 (Baccha). Type-locality: Brazil, Santa Catarina, Nova Teutônia. Distr. - same. Type q AMNH*. Ref. - Hulı, 1949e:163 (redescription), 194, fig. 11 (abdomen), 214, fig. 106 (abdomen), 216, fig. 115 (abdomen), 360, figs. $314 \& 317$ (wings). N. COMB.

PARVICORNIS (Loew), 1861:41 (Baccha), Type-locality: Cuba. Distr. U.S.A. (Florida); Cuba, Jamaica, Puerto Rico. Type o MCZ. Ref. - Hull, 1949e:250, fig. 266 (abdomen). N. COMB.

PENNAtus (Hull), 1943j:75 (Baccha). Type-locality: Panama, Canal Zone, Barro Colorado. Distr. - same. Type \& Hull. Ref. - Hull, 1949e:246, figs. 255-256 (abdomens), 266, fig. 329 (wing). N. COMB.

PERI (Hull), 1943j:75 (Baccha). Type-locality: Brazil, Santa Catarina, Nova Teutônia. Distr. - same. Type ơ AMNH*. Ref. - Hull, 1949e:228, fig. 168 (abdomen). N. COMB.

PERSIMILIS (Curran), 1930a:2 (Baccha). Type-locality: Brazil, Mato Grosso, Chapada. Distr. - Brazil (Santa Catarina, Mato Grosso). 
Type \& AMNH*. Ref. - Hull, 1949e:210, fig. 93 (abdomen). N. COMB.

PERUVIANUS (Shannon), 1927a:10 [Baccha (Pelecinobaccha)]. Type-locality: Peru, Chanchamayo. Distr. - Peru (Junin, Cuzco). Type $q$ USNM. Ref. - Hull, 1949e:206, figs. 69-70 (abdomens). N. COMB. PIIAEOPTERUS (Schiner), 1868:342 (Baccha). Type-locality: "South America". Distr. - Mexico (Guerrero, Tabasco, Vera Cruz), Guatemala, Peru, Brazil (Mato Grosso). Types o $q$ VMNH. Ref. Hull, 1949e:196, fig. 28 (abdomen). N. COMB.

PHILIPPIANUS (Enderlein), 1938:235 (Baccha; nom. nov. for flavicornis Philippi). Distr. - Chile (Curicó-Valdivia). N. COMB.

flavicornis Philippi, 1865:749 (Baccha; preoc. Loew, 1863). Typelocality: Chile, Valdivia. Types ? MNHNS.

PFillodice (Hull), 1950c:227 (Baccha). Type-locality: Peru, Chanchamayo. Distr. - same. Type of Hull. N. COMB.

P'̇OBIFER (Hull), 1943c:50 (Baccha). Type-locality: Ecuador, Oriente, Puyo, $1250 \mathrm{~m}$. Distr. - Ecuador. Type o AMNH. Ref. - Hull, 1949e:208, fig. 83 (abdomen). N. COMB.

bacchiformis Vockeroth, 1969:122, fig. 13 (wing), fig. 17 (abdomen) (Hermesomyia). Type-locality: Ecuador, Pichincha, Tandapi, $40 \mathrm{~km}$ SW Quito, 1300-1500 m. Distr. - same. Type ${ }^{*} \mathrm{CNC}^{*}$. N. SYN.

PILIPES (Schiner), 1868:342 (Bacha). Type-locality: "South America". Distr. - Brazil (Mato Grosso), Peru (Junín). Types o. o VMNH*. Ref. - Hull, 1949e:210, fig. 94 (abdomen), 272, fig. 350 (wing). N. COMB.

PINKUSI (Curran), 1939d:5 (Baccha). Type-locality: Trinidad, Tabaquite. Distr. - same. Type o AMNH*. Ref. - Hull, 1949e:242, fig. 240 (abdomen), 284, fig. 388 (wing). N. COMB.

PIRATA (Curran), 1939d:7 (Baccha). Type-locality: Brazil, São Paulo. Distr. - same. Type o AMNH*. Ref. - Hull, 1949e:218, fig. 125 (abdomen), 284, fig. 364 (wing). N. COMB.

M.Acivus (Wlliston), 1888:269 (Baccha). Type-locality: Brazil, Mato Gros-

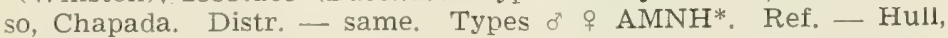
1949 e:210, fig. 90 (abdomen). N. COMB.

Pl Utonia (Hull), 1948a:2 (Baccha). Type-locality: Bolivia, Covendo. Distr. - same. Type o. USNM. Ref. - Hull, 1949e:166 (redeseription), 194, fig. 17 (abdomen). N. COMB.

Pola (Curran), 1939d:11 (Baccha). Type-locality: Brazil, Mato Grosso, Chapada. Distr. - same. Type ơ AMNH*. Ref, - Hull, 1949e: 244, fig. 249 (abdomen), 270, fig. 348 (wing). N. COMB.

POLISTA (Hull), 1943g:89 [Baccha (Mimocalla)]. Type-locality: Brazil, Santa Catarina, Nova Teutônia. Distr. - Brazil (Santa Catarina), Argentina (Buenos Aires, Tucumán). Type o AMNH*. Ref. - Hull, 1943j:65, fig. 2 (alula); 1949e:238, fig. 222 (abdomen); 236, fig. 212 (abdomen). N. COMB.

nigriventris, Bruch, 1923:1 (Baccha), misidentification.

Potentila (Hull), 1942a:100 (Baccha). Type-locality: Paraguay, Villarica. Distr. - same. Type o MCZ. Ref. - Hull, 1949e:167 (redescription), 212, fig. 102 (abdomen). N. COMB.

I'RENES (Curran), 1930a:11 (Baccha). Type-locality: Brazil, Rio de Janeiro. Distr. - same. Type o AMNH*. Ref, - Hull, 1949e:196, fig. 21 (abdomen). N. COMB. 
PinINCEPS (Hull), 1944g:56 (Baccha). Type-locality: Ecuador, Oriente, Puyo 1000 m. Distr. - Ecuador, Peru. Type o AMNH*. Ref. - Hull, 1949e:220, figs. 132, 134 (abdomen). N. COMB.

PETSCILla (Hull) 1943j:79 (Baccha). Type-locality: Argentina, Mendoza, Potrerillos. Distr. - same. Type o CU. Ref. - Hull, 1949e:168 (redescription), fig. 118 (abdomen). N. COMB.

PRcrocans (Curran), 1939d:6 (Baccha). Type-locality: Mexico, Yucatan, Chichén Itzá. Distr. - same. Type \& AMNH*. Ref. - Hull, 1949e:246, 268; figs. 254 (wing), 339 (abdomen). N. COMB.

PRUDENS (Curran), 1934b:394 (Baccha). Type-locality: Guyana, Kartabo. Distr. - same. Type of AMNH*. Ref. - Hull, 1949e:236, 254, 280 ; figs. $209 \& 210$ (abdomen), $285 \& 377$ (wing). N. COMB.

prunella (Hull), 1943j:72 (Baccha). Type-locality: Brazil, Santa Catarina, Nova Teutônia. Distr. - same. Type o AMNH*. N. COMB.

PTERoNis (Fluke), 1942:5 (Epistrophe). Type-locality: Ecuador, Tungurahua, Volcan Tungurahua, Minza Chica. Distr. - Ecuador. Type o AMNH*. Ref. - Fluke, 1950a:125, fig. 108 (male genitalia); Vockeroth, 1969:164, fig. 79 (superior lobe). N. COMB.

Pusllus (Sack), 1921:130, fig. 2 (head) (Baccha). Type-locality: Paraguay, Aregua \& St. Trinidad. Distr. - same. Types o 9 DEI \& SMF*. N. COMB

Pumilus (Austen), 1893:148, pl. 5:12 (habitus) (Baccha). Type-locality: Brazil, Region of Amazon. Distr. - Panama, Brazil. Types o $q$ $\mathrm{BM}(\mathrm{NH})$. Ref. - Hull, 1949e:206, fig. 74 (abdomen). N. COMB. PùnCTIFrons (Williston), 1891:36 (Baccha). Type-locality: Mexico, Guerrero; Omiltemi, $8000 \mathrm{ft}$; Amula, $6000 \mathrm{ft}$.; Xucumanatlán, 7000 ft.; Sierra de las Aguas Escondidas, $9500 \mathrm{ft}$. Distr. — same. Types o $\% \mathrm{BM}(\mathrm{NH}) * \& \mathrm{AMNH}^{*}$. N. COMB.

PyXIA (Hull), 1943j:68, fig. 81 (abdomen) (Baccha). Type-locality: Brazil, Santa Catarina, Nova Teutônia. Distr. - same. Type $q$ AMNH*. Ref. - Hull, 1949e:208, fig. 81 (abdomen). N. COMB.

RICUS (Curran), 1939d:6 (Baccha). Type-locality: Puerto Rico, Mayaguez. Distr. - same. Type o AMNH*. Ref. - Hull, 1949e:244, 27'2; figs. 247 (abdomen), 351 (wing). N. COMB.

RT BRICOSUS (Wiedemann), 1830:137 (Syrphus). Type-locality: "Brazil". Distr. - same. Types \& ZMB \& VMNH. N. COMB.

RUfiventris Bigot, 1883:325. Type-locality: Cuba. Distr. - same. Type $千$ $\mathrm{BM}(\mathrm{NH})$ ?

RUGOSIFRONS (Schiner), 1868:341 (Baccha). Type-locality: "South America”. Distr. - Brazil. Types o VMNH*. Ref. - Hull, 1949e:238, fig. 215 (abdomen). N. COMB.

RYL (Hull), 1943j:73, fig. 34 (abdomen) (Baccha). Type-locality: Paraguay, Villarica. Distr. - same. Type of AMNH*. Ref. - Hull, 1949e:198, fig. 34 (abdomen). N. COMB.

SAFFroNA (Hull), 19431:74 (Baccha). Type-locality: Ecuador, Manabi, Palmar. Distr. - same. Type o AMNH*. Ref. - Hull, 1949e: 208, fig. 86 (abdomen). N. COMB.

SAGITTIFERUS (Austen), 1893:144, pl 4:14 (habitus) (Baccha). Type-locality: Jamaica, Cinchona. Distr. - same. Type \& $\operatorname{BM}(\mathrm{NH})$. N. COMB.

SALPA (Hull), 1944j:40 (Baccha). Type-locality: Peru, Campamiento. Distr. - same. Type o CU. Ref. - Hull, 1949e:169 (redescription), 224, fig. 154 (abdomen). N. COMB.

SAPPHo (Hull), 1943j:69 (Baccha). Type-locality: Brazil, Santa Catarina, Nova Teutônia. Distr. - same. Type o $\mathrm{AMNH}^{*}$. Ref. - Hull, 
1949e:170 (redescription), 224, 262; figs. 157 (abdomen), 316 (wing). N. COMB.

SARGOIDES (Macquart), 1850:455 (151), pl. 14, fig. 9 (habitus), 9a (head) (Syrphus). Type-locality: "Brazil". Distr. - same. Type o OXF*. N. COMB.

SAtivus (Curran), 1941:275 (Baccha). Type-locality: Brazil, Santa Catarina, Nova Teutônia. Distr. - Colombia, Brazil (São Paulo, Santa Catarina). Type o AMNH*. Ref. - Hull, 1949e:248, 254; figs. 264 (abdomen), 283 (wing). N. COMB.

var. ARSINOE (Hull), 1949e:135, fig. 250 (abdomen) (Baccha). 'Typelocality: "Colombia". Distr. - same. Type ơ ? N. COMB.

arsenoe, Fluke, 1956:262, misspelling.

SATYRUS (Hull), 1943j:77, figs. 2 (alula), 84 (abdomen) (Baccha). Typelocality: Ecuador, Manabi, Palmar. Distr. - same. Type o AMNH*. Ref. - Hull, 1949e:208, fig. 84 (abdomen). N. COMB.

SCHWARzI (Curran), 1939d:9 (Baccha). Type-locality: Colombia, Western Cordillera, Cali Dist. Distr. - same. Type o $\mathrm{AMNH}^{*}$. Ref. Hull, 1949e:214, 278; figs. 111 (abdomen), 375 (wing). N. COMB. Scintillans (Hull), 1943h:136 (Baccha). Type-locality: Ecuador, Morro Morro. Distr. - same. Type of AMNH*. Ref. - Hull, 1949e:208, fig: 87 (abdomen). N. COMB.

SCUTELlatus Loew, 1866:39 (1872b:99). Type-locality: "Cuba". Distr. U.S.A. (Florida), Cuba, Puerto Rico, Panama, Brazil (São Paulo, Santa Catarina), Argentina (Misiones, Tucumán). Type q MCZ.

proximus Schiner, 1868:346. Type-locality: "Brazil". Type o VMNH*. N. SYN.

anthracoides Bigot, 1884b:82 (?Melanostoma). Type-locality: "Panama". Type \& OXF*. N. SYN.

loewi Sedman, in Wirth, et al,, 1965:573 (Baccha; nom. nov. for scutellatus Loew). N. SYN.

SElene (Hull), 1949e:183 (Baccha). Type-locality: Peru, Pucallpa. Distr. - same. Type ơ Hull. N. COMB.

SFPIA (Hull), 1941d:161 (Baccha). Type-locality: Brazil, São Paulo, Distr. - Brazil (São Paulo, Santa Catarina). Type o $0^{*}$ Hull. Ref. Hull, 1949e:192, fig. 10 (abdomen). N. COMB.

SERICEUS (Walker), 1837:343 (Pipiza). Type-locality: "South America". Distr. - Brazil (São Paulo), Type ơ BM(NH)*. N. COMB.

SHropshireI (Curran), 1930a:7 (Baccha). Type-locality: Panama, Canal Zone, Corozal. Distr. - same. Type of AMNH*. Ref. - Hull, 1949e:240, 282; figs. 231 (abdomen), 386 (wing). N. COMB.

SIGNIFERUS (Austen), 1893:145, pl, 4:8 (habitus) (Baccha). Type-locality: Brazil, Amazonas, Tefé (as Ega). Distr. - Guyana, Brazil. Type $\sigma^{*} \mathrm{BM}(\mathrm{NH})$. N. COMB.

SIlACEUS (Austen), 1893:149, pl. 5:13 (habitus) (Baccha). Type-locality: Brazil, Region of Amazon. Distr. - same. Type o $B M(N H)$. N. COMB.

Simulatus (Curran), 1939d:10 (Baccha). Type-locality: Peru, Lower Río Tapiche. Distr. - same. Type o AMNH*. Ref. - Hull, 1949e: 192, 272; figs. 2 (abdomen), 352 (wing). N. COMB.

SMARTI (Curran), 1939d:10 (Baccha). Type-locality: Guyana, Mazaruni. Distr. - same. Type o $\mathrm{BM}(\mathrm{NH})$. N. COMB.

Spatulatus (Giglio-Tos), 1892d:4 (Baccha). Type-locality: Mexico, Orizaba. Distr. - same. Type \& UTOR*. N. COMB.

STENOGASTER (Williston), 1888:266 (Baccha). Type-locality: Brazil, Mato Grosso, Chapada, Distr. - Mexico (Guerrero), Brazil (Mato 
Grosso), Argentina (Salta, Tucumán). Type ơ AMNH*. Ref. Hull, 1949e:232, 266, 272; figs. 189 \& 190 (abdomen), 331 \& 357 (wing). N. COMB.

STIPA (Hull), 1949e:110 (Baccha) [also, 1943j:94, fig. 71 (abdomen)]. Type-locality: "Colombia". Distr. - same, Type ? ? N. COMB.

STOLO (Walker), 1852:241 (Syrphus). Type-locality: "Brazil". Distr. same. Type $\sigma^{*} \mathrm{BM}(\mathrm{NH})$. N. COMB.

StRIATUS (Walker), 1852:221 (Ascia). Type-locality: "Brazil". Distr. same. Type of BM(NH). Ref. - Hull, 1949e:198, fig. 32 (abdomen). N. COMB.

SUBCHALYBeus (Walker), 1857:157 (Syrphus). Type-locality: "Valley of Amazon". Distr. - same. Type ? BM(NH). N. COMB.

Summus (Fluke), 1936:59, fig. 1 (abdomen) (Baccha). Type-locality: Brazil, Guanabara, Rio de Janeiro, Jardim Botânico. Distr. same. Type ${ }^{*}$ AMNH*. Ref. - Hull, 1949e:214, fig. 13 (abdomen), 276, fig. 368 (wing), N. COMB.

Susio (Hull), 1941c:61 (Baccha). Type-locality: Brazil, São Paulo, Avanhandava. Distr. - same. Type o Hull. Ref. - Hull, 1949e:171 (redescription), 220, fig. 138 (abdomen). N. COMB.

TARSALIS (Walker), 1837:345 (Syrphus). Type-locality: "South America". Distr. - same. Type ? BM(NH). N. COMB.

meridionalis Goot, 1964:215 (Syrphus, unjustified nom. nov. for tarsalis Walker; preocc. Fluke, 1950). N. SYN.

TELESCOPICUS (Curran), 1930a:4 (Baccha). Type-locality: Panama, Canal Zone, Barro Colorado Island. Distr. - same. Type of AMNH*. Ref. - Hull, 1949e:204, 206, 276; figs. 60 \& 71 (abdomen), 369 (wing); Hull, 1943j:44, fig. 1 (alula). N. COMB.

TENUIS (Walker), 1852:222 (Baccha). Type-locality: "Brazil". Distr. same. Type \& $\mathrm{BM}(\mathrm{NH})$. N. COMB.

THECLA (Hull), 1943j:67 (Baccha). Type-locality: Brazil, Santa Catarina, Nova Teutônia. Distr. - same; Type o AMNH*. N. COMB.

TIARELla (Hull), 1944g:57 [Baccha (Pipunculosyrphus)]. Type-locality: Brazil, Santa Catarina, Nova Teutônia. Distr. - same. Type q AMNH*. Ref. - Hull, 1949e:226, 256; figs. 165 \& 166 (abdomen), 296 (wing). N. COMB.

TITANIA (Hull), 1943j:70, fig. 51 (abdomen) (Baccha). Type-locality: Brazil, Santa Catarina, Nova Teutônia. Distr. - Brazil (Santa Catarina), Argentina (Salta, Tucumán). Type o AMNH*. Ref. Hull, 1949e:202, fig. 51 (abdomen). N. COMB.

TRABIS (Fluke), $1942: 5$, fig. 6 (abdomen) (Epistrophe). Type-locality: Ecuador, Tungurahua, Volcan Tungurahua, Runtun. Distr. Ecuador, Argentina (Tucumán). Type o $\mathrm{AMNH}^{*}$. Ref. - Fluke, 1950a:125, fig. 109 (male genitalia); Vockeroth, 1969:165, fig. 81 (superior lobe). N. COMB.

TRANSATlanticus (Schiner), 1868:343 (Baccha). Type-locality: "Brazil \& Colombia". Distr. - "Colombia", Peru, Brazil. Types ot o VMNH*. Ref. - Hull, 1949e:216, 284; figs. 119 \& 120 (abdomen), 392 \& 393 (wing). N. COMB.

tricinctus (Bigot), 1883b:333 (Baccha). Not neotropical; Fluke, 1956: 265 , error for tricinctus (Wulp).

TRILOBUS (Hull), 1944b:11 (Baccha). Type-locality: "Amazon". Distr. same. Type \& $\mathrm{BM}(\mathrm{NH})$. Ref. - Hull, 1949e:172 (redescription), 244, 258; figs. 153 (abdomen), 198 (wing). N. COMB.

TRINIDADENSIS (Curran), 1939d:11 (Baccha). Type-locality: Trinidad, Ta- 
baquite. Distr. - same. Type \& AMNH*. Ref. - Hull, 1949e:212, 254; figs. 98 (abdomen), 284 (wing). N. COMB.

TRISTIS (Hull), 1930:140, fig. 7 (abdomen) (Baccha). Type-locality: Mexico, Vera Cruz, Jalapa, $4400 \mathrm{ft}$. Distr. - same. Type o ANSP. Ref. - Hull, 1949e:173 (redescription), 194, fig. 19 (abdomen). N. COMB.

ULPICA (Hull), 1957:188 (Baccha). Type-locality: Peru, Chanchamayo. Distr. - same. Type $q$ Hull. N. COMB.

URANIA (Hull), 1949e:185 (Baccha). Type-locality: Peru, Pucallpa. Distr. - same. Type o Hull. N.COMB.

URSula (Hull), 1947b:234 (Baccha). Type-locality: Paraguay, Villarica. Distr. - same. Type ot Hull. Ref. - Hull, 1949e:220, 226, 284; figs. 140 \& 161 (abdomen), 389 (wing). N. COMB.

VAMPYRUS (Hull), 1943j:98, fig. 95 (abdomen); 1948a:7 (Baccha). Typelocality: Brazil, São Paulo. Distr. - same. Type o Hull. Ref. Hull, 1949e:210, fig. 95 (abdomen). N. COMB.

VANDA (Hull), 1943h:136 (Baccha). Type-locality: Brazil, Santa Catarina, Nova Teutônia. Distr. - same. Type ơ AMNH*. Ref. - HulI, 1949e:174 (redescription), 214, 222, 262; figs. 110 \& 148 (abdomen), 315 \& 318 (wing). N. COMB.

vanessa (Hull), 1949e:190 (Baccha). Type-locality: Peru, Pucallpa. Distr. - same. Type o Hull. N. COMB.

VARIEGATUS (Macquart), 1842:168 (108), pl. 18, fig. 4 (habitus) 4a (head) $($ Bacch $a)$. Type-locality: Brazil, Paraná, Guaratuba. Distr. -

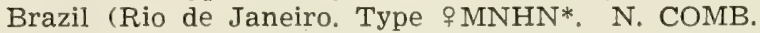

VERA (Hull), 1943j:50, fig. 43 (abdomen) (Baccha) [also, 1944b:10]. Typelocality: "Amazon" [1944b:10]. Distr. - same. Type o BM(NH). Ref. - Hull, 1949e:200, fig. 43 (abdomen), 272, fig. 353 (wing). N. COMB.

VERONA (Curran), 1941:272 (Baccha). Type-locality: Colombia, Vista Nieve. Distr. - same. Type \& AMNH*. Ref. - Hull, 1949e:240, 254; figs. 225 (abdomen), 288 (wing). N. COMB.

vespuccius (Hull), 19431:73 (Baccha). Type-locality: Brazil, Santa Catarina, Nova Teutônia. Distr. - same. Type o AMNH*. Ref. Hull, 1949e:192, fig. 1 (abdomen). N. COMB.

victoria (Hull), 1941j:181 (Baccha). Type-locality: Brazil, São Paulo. Distr. - same. Type o Hull, MZUSP. Ref. - Hull, 1949e:196. fig. 27 (abdomen), N. COMB.

VIERECKI (Curran, 1930a:6 (Baccha). Type-locality: Panama, Canal Zone, Barro Colorado Island. Distr. - Panama, Colombia (Santa Maria). Type o $\mathrm{AMNH}^{*}$, Ref. - Hull, 1949e:236, fig. 213 (abdomen). N. COMB.

violaceus (Hull), 1943j:71 (Baccha). Type-locality: Brazil, Santa Catarina, Nova Teutônia. Distr. - same. Type o AMNH*. Ref. - Hull, 1949 e:220, fig. 135 (abdomen). N. COMB.

virga (Fluke), 1942:7, fig. 9 (abdomen) (Epistrophe). Type-locality: Ecuador, Imbabura, Cuicocha, 3200 m. Distr. - same. Type o AMNH*. Ref. - Vockeroth, 1969:164, fig. 82 (superior lobe). N. COMB.

virgilio (Hull), 1942d:46 (Baccha). Type-locality Brazil, Paraná. Distr. - same. Type o Hull. Ref. - Hull, 1949e:204, fig. 63 (abdomen). N. COMB.

virginio (Hull), 1941j:182 (Baccha). Type-locality: Brazil São Paulo, Osasco. Distr. - Brazil. Type or Hull. Ref. - Hull, 1949e:244, fig. 244 (abdomen). N. COMB. 
VitTiger (Hull), 1949c:735 [also, 1949e:123, figs. 245 (abdomen), 384 (wing)] (Baccha). Type-locality: Guyana, Essequibo River, Moraballi Creek. Dark Forest. Distr. - Guyana, Brazil (São Paulo). Type \& $\mathrm{BM}(\mathrm{NH})$. N. COMB.

volCaNus (Fluke), 1942:6, fig. 8 (abdomen) (Epistrophe). Type-locality: Panama, Chiriquí, El Volcan. Distr. - Panama, Ecuador. Type ơ $\mathrm{AMNH}^{*}$. N. COMB.

Wiedemanni Enderlein, 1938:234. Type-locality: "Brazil". Distr. - same. Type \& ZMB. Ref. - Wiedemann, 1830:124 [p.p. flavipennis Wiedemann].

WIL.tistonI Thompson (nom. nov. for dolosa Williston). Distr. - "Mexico", Ref. - Hull, 1949e:234, fig. 202 (abdomen). NOM. NOV.

dolosa Williston, 1891:37, pl. 1:7 (habitus), $7 \mathrm{a}+\mathrm{b}$ (head) (Baccha; preocc. Walker, 1857). Type-locality: Mexico, Guerrero: Omiltemi, $8000 \mathrm{ft}$.; Xucumanatlan, $7000 \mathrm{ft}$; Amula, $6000 \mathrm{ft}$; Guatemala, Cerro Zunil, 4000-5000ft. Types o o (BM(NH) \& AMNH*.

Wilhelmina (Doesburg), 1963:27, fig. 5 (habitus), 5a+b (head) (Baccha). Type-locality: Surinam, Paramaribo. Distr. - same. Type o Doesburg. N. COMB.

WULPIANUS (Lynch Arribálzaga), 1891:250 (1892c:47) (Baccha, nom. nov. for tricinctus Wulp). Distr. - Argentina. N. COMB.

tricinctus Wulp, 1888: 376, pl. 10, fig. 8 (habitus). (Baccha; preocc. Bigot, 1883). Type-locality: Argentina, Prov. 'Tucumán. Types ơ lost?.

XANTHOPTERUS (Wiedemann), 1830:120 (Syrphus). Type-locality: Brazil. Distr. - same. Type of SMF*. N. COMB.

xantipPe (Hull), 1949e:177 (Baccha). Type-locality: Peru, Pucallpa. Distr. - same. Type of Hull. N. COMB.

zabulon (Hull), 1949b:244 (Baccha). Type-locality: Peru, Chanchamayo. Distr. - same. Type o Hull. N. COMB.

ZENIA (Curran), 1941:266 (Baccha). Type-locality: Brazil, Santa Catarina Nova Teutônia. Distr. - same. Type o AMNH*. Ref. Hull, 1949e: 250, fig. 273 (abdomen). N. COMB.

ZENillia (Curran), 1941:274 (Baccha). Type-locality: Brazil, Santa Catarina, Nova Teutônia. Distr. - same. Type ơ AMNH*. Ref. Hull, 1949e:250, 258; figs. 83, 272 (abdomen), 305 (wing).

zinnia Hull, 1943j:78, fig. 2 (alula), 82 (abdomen) (Baccha). Typelocality: Brazil, Santa Catarina, Nova Teutônia. Type q AMNH*. N. SYN.

ZEPHYREUS (Hul1), 1947b:232 (Baccha). Type-locality: Paraguay, Villarica. Distr. - same. Type o USNM. Ref. - Hull, 1949e: 232, 274; figs. 188 (abdomen), 363 (wing). N. COMB.

ZERENE (Hull), 1949b:245 (Baccha). Type-locality: Peru, Chanchamayo. Distr. - same. Type o Hull. N. COMB.

ZETEKI (Curran), 1930a:8 (Baccha). Type-locality: Panama, Canal Zone, Barro Colorado Island. Distr. - Honduras, Panama, Brazil (Mato Grosso). Type o* AMNH*. Ref. - Hull, 1949e:175 (redescription), 204, fig. 66 (abdomen). N. COMB.

zILlA (Hull), 1943m:214 (Baccha). Type-locality: Brazil, Santa Catarina, Nova Teutônia. Distr. - Ecuador, Brazil (Santa Catarina). Type o AMNH*. Ref. - Hull, 1949e:248, fig. 262 (abdomen). N. COMB.

zITA (Curran), 1941:273 (Baccha). Type-locality: Brasil, Santa Catarina, Nova Teutônia. Distr. - Brazil (Santa Catarina), Argentina 
(Tucumán). Tvpe o AMNH*. Ref. - Hull, 1943j:65, fig. 2 (alula); Hull, 1949e:200, 252; figs. 45 (abdomen), 275 (wing). N. COMB. var. fuscus Hull, 1949e:252, fig. 274 (abdomen) (Baccha). Nomen nudum [q $\left.\mathrm{AMNH}^{*}\right]$.

zOBEIDE (Hull), 1943g:90 (Baccha). Type-locality: Brazil, Santa Catarina, Nova Teutônia. Distr. - same. Type ơ AMNH*. Ref. - Hull, 1949e:230, fig. 182 (abdomen), N. COMB.

ZOROASTER (Hull), 1943i:76, fig. 2 (alula), 80 (abdomen) (Baccha). Typelocality: Brazil, Santa Catarina, Nova Teutônia. Distr. - Brazil (Santa Catarina), Argentina (Tucumán). Type o AMNH*. Ref. Hull, 1949e:208, fig. 80 (abdomen). N. COMB.

\section{Genus Salpingogaster Schiner}

Amathia Walker, 1852:223 (preocc. Lamouroux, 1812; Rour, 1828; Duponchel, 1829; Rathke, 1837). Type-species, costalis Walker (mon.). SALPINGOGASTER Schiner, 1868:344. Type-species, pygophora Schiner (orig. des.).

Flexineura Bezzi, 1908:294, nomen nudum.

Ref. - Sack, 1920 (rev. key); Curran, 1941 (key); Knutson, 1971 (biol.).

\section{Subgenus Salpingogaster Schiner}

ABDominalis Sack, 1920:242, fig. D (tip of abdomen). Type-locality: "Bolivia". Distr. - same. Type o MD.

Bequaerti Curran, 1933:2. Type-locality: Guatemala, Sa. Emilia, Pochuta. Distr. - same. Type o AMNH*.

BICOLOR Sack, 1920:250, fig. m (abdomen). Type-locality: Bolivia, La Paz, Mapiri, 2000 m. Distr. - same. Type o MD.

BIPUNCTIFRoNS Curran,, 1934b:397. Type-locality: Guyana, Kartabo. Distr. - same. Type o AMNH*.

BRownI Curran, 1941:286. Type-locality: Ecuador, Tungurahua, Volcan Tungurahua, Minza Chica. Distr. - Ecuador. Type o AMNH*. Ref. - Hull.1949d:297, fig. 11L (wing).

maculipennis Hull, 1944a:166. Type-locality: “?South America". Type १VMNH*. N. SYN.

BRUNerr Curran, 1932a:6. Type-locality: Cuba, Sierra Maestra. Distr. same. Type of AMNH*.

CORNutA Hull, 1944a:168. Type-locality: "Venezuela". Distr. - same. Type o VMNH.

costalis (Walker), 1852:223 (Amathia). Type-locality: Brazil. Distr. same. Type o BM(NH). Ref. - Sack, 1920:253 (redescription).

COTHurnata Bigot, 1883:329. Type-locality: "Mexico". Distr. - same. Type o BM(NH) ? Ref. - Sack, 1920:246 (redescription).

DianA Hull, 1943f:33. Type-locality: "Brazil". Distr. - same. Type \& Hull. FRCNTALIS Sack, 1920:243, fig. e (male abdominal tip), f (female abdomen). Type-locality: Bolivia, Coroico, $1000 \mathrm{~m}$. Distr. - same. Types of MD.

GRACILIS Sack, 1920:251, figs. $\mathrm{n}+\mathrm{o}$ (head), $\mathrm{p}$ (mesonotum), $\mathrm{q}$ (tip of male abdomen). Type-locality: Peru, Vilcanota. Distr. - Peru, Brazil (Santa Catarina). Types of o UNMB.

Halcyon Hull, 1940b:434. Type-locality: Panama, Canal Zone, Barro Colorado Island. Distr. - Panama, Brazil (Santa Catarina), Argentina (Formosa). Type o Hull. 
compressa Curran, 1941:284. Type-locality: Brazil, Santa Catarina, Nova Teutônia. Type ơ AMNH*. N. SYN.

IMPURA Curran, 1941:285. Type-locality: Brazil, São Paulo. Distr. - same. Type o AMNH*.

LIMBIPENNIS Williston, 1891:29. Type-locality: Mexico, Guerrero, Amula, $6000 \mathrm{ft}$. Distr. - same. Types o BM(NH). Ref. — Sack, 1920:256 (redescription).

limbippennis, Sack, 1920:256, misspelling.

LineATA Sack, 1920:253, figs. $r+s$ (male abdomen). Type-locality: Bolivia, La Paz, Mapiri. Distr. - same. Type o MD.

MiNor Austen, 1893:161, pl. 5:4 (habitus). Type-locality: Brazil, Pará. Distr. - same. Type o BM(NH). Ref. - Sack, 1920:247 (redescription).

NIGRA Schiner, 1868:344, pl. 4:4 (habitus), 4a (wing). Type-locality: "South America". Distr. - Mexico, Guatemala, Panama, Brazil, Peru, Argentina (Jujuy, Tucumán). Types o o VMNH*. Ref. - Sack, 1920:254 (redescription), figs. a (wing), t, u, \& v (abdomen).

anchoratus Bigot, 1883:328. Type-locality: “Mexico". Type o BM(NH)? NIGRICAUDA Sack, 1920:248, fig. h (head). Type-locality: Bolivia. Distr. same. Types $\sigma^{*}$ क UNMB.

Nova Giglio-Tos, 1892c:7. Type-locality: Mexico. Distr. - same. Type $q$ UTOR*.

Panamana Hull, 1944c:33. Type-locality: Panama, Bella Vista. Distr. same. Type o MCZ.

pannamana, Fluke, 1957:6, misspelling.

PESSUlagyna Hull, 1941f:48. Type-locality: Panama, Canal Zone, Barro Colorado Island. Distr. - same. Type ơ Hull.

Petiolata Hull, 1944a:165. Type-locality: Bolivia, Rio Songo. Distr. same. Type o $\mathrm{VMNH}^{*}$.

PUNCTIFRons Curran, 1929:493. Type-locality: "Cuba". Distr. - U.S.A. (Florida). Type $\sigma^{*}$ Stanford Univ., Calif.

PYGOPHORA Schiner, 1868:344. Type-locality: "South America". Distr. Cuba, Panama, Mexico, Brazil. Types o VMNH*. Ref. — Sack, 1920:239 (redescription), fig. b (tip of male abdomen); Hull, 1949d:264, fig. 3e (head).

Relrcta Curran, 1941:285. Type-locality: Cuba, Vinales, Distr. - Cuba. Type o" AMNH*.

STIGMATIPEN Nis Hull, 1940a:250. Type-locality: "Venezuela". Distr. - same. Types o VMNH \& Hull.

UNCINATA Hull, 1945a:216. Type-locality: Costa Rica, La Suiza. Distr. same. Type $\sigma$ USNM.

urania Hull, 1949i:232. Type-locality: Peru, Chanchamayo. Distr. - same. Type $\sigma^{*}$ Hull.

VERA Hull, 1944a:169. Type-locality: Bolivia, Río Songo. Distr. - same. Type o VMNH.

virgata Austen, 1893:159, pl. 5:4 (habitus). Type-locality: Brazil, Region of Amazon. Distr. - Brazil, Bolivia. Types of $q \mathrm{BM}(\mathrm{NH})$. Ref, Sack, 1920:240 (redescription), fig. c (head).

\section{Subgenus Eosalpingogaster Hull}

EOSALPINGCGAStER Hull, 1949d:299 (as genus). Type-species, Baccha conopida Philippi (orig. des.).

CONOPIDA (Philippi), 1865:750 (Baccha). Type-locality: Chile, Santiago. Distr. - Brazil (São Paulo), Chile, Argentina. Types MNHNS. 
Ref. - Sack, 1920:244 (redescription), fig. g (head) [as macula Schiner]; Knutson, 1971:34 (description of puparium), figs. $3+4$ (puparium).

?nigriventris Bigot, 1883:329. Type-locality: Uruguay, Montevideo.

Type o $\mathrm{BM}(\mathrm{NH})$. Ref. - Sack, 1920:249 (redescription), figs. j

(head), $\mathrm{k}$ (mesonotum); Sack, 1921:134, figs. 7 (mesonotum), 8 (puparium).

macula Schiner, 1868:345. Tpe-locality: "Chile". Types o + VMNH*. nigroventris, Williston, 1886:315, misspelling:

mimetica Brèthes [not Lahille], 1907:292 (Baccha). Nomen nudum.

DACTYlopianA Blanchard, 1938:348, figs. 2a (puparium), b (head), c (mesonotum), d (abdomen). Type-locality: Argentina, Los Armadores, Catamarca \& Entre Ríos. Distr. - same. Types o [author's coll.]. LIPOSETA Fluke, 1937:10, figs. 12 (wing), 13 (thorax). Type-locality: Ecuador, Puerto Grande, Isla Puná. Distr. - Ecuador, Argentina (Tucumán, La Rioja, Río Negro). Type \& AMNH*.

\section{Genus Allograpta Osten Sacken}

ALlograpta Osten Sacken, 1875:49. Type-species, Scaeva obliqua Say (mon.). Claraplumula Shannon, 1927a:8. Type-species, latifacies Shannon (orig. des.).

Fazia Shannon, 1927a:25. Type-species, bullaephora Shannon (orig. des.).

Oligorhina Hull, 1937b:30 (preocc. Fairmaire \& Germain, 1863). Typespecies, aenea Hull (orig. des.).

Chasmia Enderlein, 1938:213 (preocc. Enderlein, 1922). Type-species, hians Enderlein (orig. des.).

Rhinoprosopa Hull, 1942f:24 (nom. nov, for Oligorhina Hull). Type-species. Oligorhina aenea Hull (aut.). N SYN.

Microsphaerophoria Frey, 1946:168. Type-species, plaumanni Frey (orig. des.).

Nieoscaeva Frey, 1946:170. Type-species, Syrphus aeruginosifrons Schiner (orig. des.). N. SYN.

Epistrophe, subg. Metallograpta Hull, 1949d:293. Type-species, Allograpta colombia Curran (orig. des.).

Epistrophe, subg. Metepistrophe Hull, 1949d:293. Type-species, remigis Fluke (orig. des.) [see Fluke, 1951c:218; Vockeroth, 1969:126].

Antillus Vockeroth, 1969:130. Type-species, ascitus Vockeroth (orig. des.). N. SYN.

AENEA (Hull), 1937b:31 (Oligorhina). Type-locality: Haiti, Mt. La Hotte, Desbarrière. Distr. - same. Type o MCZ. Ref. - Hull, 1949e:222, figs. $142 \& 147$ (abdomen); Hull, 1949d:288, fig. 9d (head). N. COMB.

AERUGINOSIFRONS (Schiner), 1868:352 (Syrphus). Type-locality: "South America". Distr. - Brazil. Types or \& VMNH*. N. COMB.

ALtA Curran, 1936b:15. Type-locality: Colombia, Popayán. Distr. - Colombia; Ecuador. Type o AMNH*. Ref. - Fluke, 1942:23, fig. 31 (abdomen); Fluke, 1950a:129, fig. 134 (male genitalia).

flavomaculata Hull, 1937a:169. Type-locality: Colombia, Bogotá. Type on USNM.

ALtissima (Fluke), 1942:10, fig. 17 (abdomen) (Epistrophe). Type-locality: Ecuador. Type o AMNH*. Ref. - Fluke, 1950a:127, figs. 124 \& 130 (male genitalia, as remigis).

ANNULipes (Macquart), 1850:464 (160), pl. 14, fig. 18 (head) (Paragus). 
Type-locality: Brazil, Bahia. Distr. - Colombia, Ecuador, Peru, Brazil (Bahia, São Paulo), Bolivia. Type o MNHN*. N. COMB. scutellata Sack, 1941:99 (1951:95). Type-locality: Peru, Pichis; Bolivia, Mapiri. Type $0^{*}+$ MD?. N. SYN.

geminata Fluke, 1942:16, fig. 34 (abdomen). Type-locality: Ecuador, Río Pastaza, Santa Inés. Type o AMNH*.

APERTA Fluke, 1942:19, fig. 48 (abdomen). Type-locality: Guyana, Kaieteur, Savannah. Distr. - Surinam, Guyana. Type $\sigma^{*} \mathrm{AMNH}^{*}$.

var. NOTATA Doesburg, 1966:64. Type-locality: Surinam, Wilhelmina Mts., Camp 3. Distr. - same. Types o Doesburg.

ARgENTIPIla (Fluke), 1942:13, fig. 30 (abdomen) (Epistrophe). Typelocality: Ecuador, Oriente, Puyo, 1000 m. Distr. - Ecuador, Peru, Argentina (La Rioja). Type $\sigma^{*} \mathrm{AMNH}^{*}$. Ref. - Fluke, 1950a:127, fig. 131 (male genitalia).

ARMillata (Fluke), 1942:12, fig. 23 (abdomen), 24 (head) (Epistrophe). Type-locality: Ecuador, Bolivar, Hda. Talahua. Distr. - same.

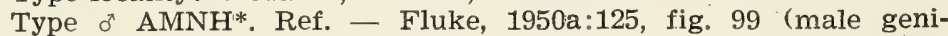
talia).

ASCITA (Vockeroth), 1969:130, fig. 18 (abdomen) (Antillus). Type-locality: Haiti, Desbarrière, Mt. La Hotte, nr, 4000 ft. Distr. - same. Type o $\mathrm{MCZ}^{*}$. N. COMB.

BICINCTA (Bigot), 1884b:112 (Mesograpta?). Type-locality: Mexico. Distr. - same. Type o OXF*. N. COMB.

BILINEAta Enderlein, 1938:220. Type-locality: Argentina, Prov. Buenos Aires. Distr. - same. Type o ZMB?

BILINEella Enderlein, 1938:219. Type-locality: Colombia, "Cordilliere, terra caliente". Distr. - same. Type o ZMB.

BISINTERRPTA (Enderlein), 1938:210 ( $F a z i a)$. Type-locality: Colombia, "Cordilliere, terra fria". Distr. - same. Type o. ZMB. N. COMB.

BRownI Fluke, 1942:17, figs. $41 \& 42$ (head), 43 (abdomen). Type-locality: Ecuador, Imbabura, Cuicocha, $3200 \mathrm{~m}$. Distr. - same. Type ot AMNH*.

BULlAEPHORA (Shannon), 1927a:25, figs. 8 (head), 10 (wing tip) (Fazia). Type-locality: Chile. Distr. - Ecuador, Chile (Coquimbo - Valdivia). Type o USNM*. Ref. - Fluke, 1942:23, fig. 22 (abdomen).

Colombia, Curran, 1925c:349. Type-locality: Colombia, Sierra Quemada. Distr. - Colombia, Ecuador, Brazil, Argentina (Tucumán, Salta). Type o CU*. Ref. - Fluke, 1942:12 (redescription), figs. 25 (head), 26 (abdomen); 1950a:129, fig. 135 (male genitalia).

CUBANA Curran, 1932a:3. Type-locality: "E.E.A. [Estación Experimental Agricola] de Cuba". Distr. U.S.A. (Florida); Bahamas, Cuba. Type o AMNH*. Ref. - Fluke, 1942:24, fig. 39 (abdomen).

DELimbata Enderlein, 1938:224. Type-locality: Colombia, Bogotá. Distr. same. Types o ZMMB.

DUPlofasciata Enderlein, 1938:217. Type-locality: Mexico, Mexico State. Distr. - same. Type o ZMB or DEI.

Eupeltata (Bigot), 1884b:91 (Syrphus). Type-locality: "Mexico". Distr. Mexico (Chiapas). Types ox OXF*.

EXотіCA (Wiedemann), 1830:136 (Srphus). Type-locality: "Brazil". Distr. U.S.A. (Oregon to Nebraska), south to Chile (Antofagasta) and Argentina (Catamarca). Types $\sigma^{*}+\mathrm{VMNH}^{*}$ \& ZMB. Ref. Fluke, 1942:24, fig. 47 (abdomen); 1950a:129, figs. 136 \& 138 (male genitalia). 
quadrigemina (Thomson), 1869:500 (Syrphus). Type-locality: Argentina, Buenos Aires. Type of NRS*.

fracta Osten Sacken, 1877:331. Type-locality: U.S.A., California, Santa Monica. Type ot MCZ.

skottsbergi Enderlein, 1940:663. Type-locality: Chile, Juan Fernández Islands, Masatierra \& Masafuera. Types $\sigma^{*}$ \& NRS.

FAlCATA Fluke, 1942:16, fig. 36 (abdomen). Type-locality: Brazil, Santa Catarina, Nova Teutônia. Distr. - Colombia (Putamayo River), Venezuela (Amazonas), Ecuador, Peru (Cuzco), Brazil (Santa Catarina), Argentina (Tucumán). Type o $\mathrm{AMNH}^{*}$.

FASciata Curran, 1932a:4. Type-locality: "Peru". Distr. - Ecuador, Peru. Type \& AMNH*. Ref. - Fluke, 1942:23, figs. 28 (head), 29 (abdomen); 1950a:129, fig. 137 (male genitalia).

FASCIFRoNS (Macquart), 1846:265 (137), pl. 11, fig. 11 (wing) (Syrphus). Type-locality: "Nouvelle Grenade". Distr. - same. Type ơ OXF*. N. COMB.

FLAvibuCA Enderlein, 1938:216. Type-locality: "Paraguay". Distr. - Colombia, Brazil, Paraguay, Uruguay, Argentina. (Buenos Aires, Salta). Types of o ZMB.

var. INTERRUPtA Enderlein, 1938:216. Type-locality: Uruguay, Montevideo. Types $\sigma^{*}$ 우 ZMB.

FLANOPHYLLA (Hull), 1943h:319 (Rhinoprosopa). Type-locality: Peru, Perené Valley. El Campamiento. Distr. - same. Type o CU. Ref. Hull, 1949d:269, fig. 6b (head); 1949e:222, fig. 146 (abdomen). N. COMB.

FLUker Curran, 1936b:13. Type-locality: Cuba, Oriente, Sierra del Cobre, Loma del Gato. Distr. - same. Type o* AMNH*.

FORRERI (Giglio-Tos), 1893:32 (Sphaerophoria). Type-locality: Mexico, Durango, La Ciudad; Solco, Cuernavaca. Distr. - same. Types $\sigma^{\pi}$ UTOR*. N. COMB.

FUNERALIA (Hull), 1944c:27 (Epistrophe). Type-locality: Jamaica, New Castle. Distr. - same. Type o MCZ.

Fuscisquama Curran, 1927b:4. Type-locality: Puerto Rico. Ensenada. Distr. - Puerto Rico, Virgin Islands. Type o $\mathrm{AMNH}^{*}$. Ref. - Fluke, 1942:24, fig. 35 (abdomen).

HARLEQUiNA Hull, 1949a:76. Type-locality: Chile, Santiago. Distr. - same. Type or Hull.

Fistata Fluke, 1942:17, fig. 37 (abdomen). Type-locality: Brazil, São Paulo. Distr. - Peru (Cuzco), Brazil (São Paulo). Type ơ AMNH*.

HERMOSA (Hull), 1941f:48 (Fazia). Type-locality: Brazil, São Paulo. Distr. - Brazil (Rio de Janeiro, São Paulo, Santa Catarina). Type o Hull. Ref. - Fluke, 1942:8 (redescription), fig. 14 (abdomen); 1950a:125, fig. 110 (male geintalia).

brunneola Frey, 1946:170 (Neoscaeva; as aeruginosifrons var.) Typelocality: Brazil, Rio de Janeiro, Niterói. Type of UZMH*. N. SYN.

HIANS (Enderlein), 1938:213 (Chasmia). Type-locality: Mexico, Guadalajara. Distr. - same. Type o ZMB. N. COMB.

HORTENSIS (Philippi), 1865:746 (Syrphus). Type-locality: Chile, Santiago, Valdivia. Distr. - Peru, Chile (Coquimbo - Magallanes), Argentina (Neuquén). Type(s) ? MNHNS. Ref. - Shannon, 1927a:24 (redescription); Shannon \& Aubertin, 1933:131, figs. 24a \& b (heads), 25a (abdomen); Fluke, 1942:24, fig. 50 (abdomen). 
var. BIMACUlata Enderlein, 1938:223. Type-locality: Chile, Comudes. Type $0^{*}$ ZMB.

var. QUADRIMACULATA Enderlein, 1938:223. Type-locality: Chile, Comudes. Types $\sigma^{\pi} \mathrm{ZMB}$.

IMITATOR (Curran), 1925c:351 (Epistrophe). Type-locality: Colombia, Vista Nieve, San Lorenzo Mt. Distr. - Colombia, Ecuador, Bolivia. Type o CU*. Ref. - Fluke, 1942:23, figs. 20 (head), 21 (abdomen).

LATIFACIES (Shannon), 1927a:8 (Claraplumula). Type-locality: Peru, Tincochaca, $7000 \mathrm{ft}$. Distr. - Ecuador, Peru. Type $\delta^{*}$ USNM*. Ref. Fluke, 1942:22, figs. 4 (head), 5 (abdomen); 1950a:127, figs. 127 \& 125 (male genitalia); Vockeroth, 1969:165, fig. 86 (male genitalia).

LiMBatA (Fabricius), 1805:251 (Scaeva). Type-locality: "Americae Insulis". Distr. - West Indies, Brazil (São Paulo, Paraná). Type o MC.

LUCIFERA (Hull), 1943m:216 (Rhinoprosopa). Type-locality: Ecuador, Pinas, $1600 \mathrm{ft}$. Distr. - same. Type o* AMNH*. Ref. - Hull, 1949e:222, fig. 141 (abdomen). N. COMB.

LUNA (Fluke), 1942:8, figs. 12 (abdomen), 13 (head) (Epistrophe), Typelocality: Ecuador, Tungurahua,. Volcan Tungurahua, Minza Chica. Distr. - Ecuador. Type o" AMNH*. Ref. - Fluke, 1950a:129, fig. 139 (male genitalia).

MACQUARTI (Blanchard), 1852:411 (Syrphus). Type-locality: Chile, Serena. Distr. - Chile (Santiago - Malleco), Argentina (Tucumán, La Rioja, Mendoza). Type(s) ? MNHN.

australis Shannon, 1927a:26, fig. 7 (head) (Fazia). Type-locality: "Chile". Type o" USNM.

MEXiCANA (Enderlein), 1938:212 (Fazia), Type-locality: "Mexico". Distr. same. Type o ZMB.

MICRURA (Osten Sacken), 1877:330 (Sphaerophoria). Type-locality: U.S.A., California, The Geysers, Sonoma County; San Rafael; Brooklyn, near San Francisco. Distr. - U.S.A. (California, Washington, Texas); Mexico (Durango, Mexico City), Guatemala, Honduras, Ecuador. Types o MCZ. Ref. - Fluke, 1942:23, fig. 32 (abdomen); 1950a:129, fig. 140 (male genitalia).

picticauda Bigot, 1884b:102 (Sphaerophoria). Type-Iocality: Mexico. Types $\sigma^{*}$ \& $\mathrm{OXF}^{*}$.

MU (Bigot), 1884b:105 (Mesograpta?). Type-locality: "Mexico". Distr. same. Type \& OXF*.

MunozI Enderlein, 1938:222. Type-locality: Colombia, Bogotá. Distr. same. Type o ZMB.

NASIGERA (Enderlein), 1938:212 (Fazia). Type-locality: "Colombia". Distr. - Colombia. Types or o ZMB. N. COMB.

NASUTA (Bigot), 1884b:103 (Sphaerophoria). Type-locality: "Mexico". Distr. - Mexico (Durango, Chiapas). Type $0^{*} \mathrm{OXF}^{*}$. N. COMB.

nasuta Bigot, 1888:253 (Sphaerophoria; preocc. Bigot. 1884). Typelocality: "Mexico". Type ot OXF*. N. SYN.

NEOTROPICA Curran, 1936b:14. Type-locality: Colombia, Popayán. Distr. Colombia, Ecuador, Brazil (São Paulo, Santa Catarina), Argentina (Tucumán). Type o AMNH*. Ref. - Fluke, 1942:24, fig. 49 (abdomen); 1950a:128, fig. 41 (male genitalia).

OBLIQUA (Say), 1823:89 (Sca€va). Type-locality: "United States". Distr. Canada (Quebec) west to U.S.A. (Oregon), south to Brazil (Santa Catarina). Type(s) ? lost. Ref, - Fluke, 1942:24, fig. 46 (abdomen); Vockeroth, 1969:165, fig. 85 (male genitalia). 
var. DEJoNGi Doesburg, 1958:44. Type-locality: "Curaçao". Distr. same. Type ot Doesburg.

PIURANA Shannon, 1927a:9, fig. 4 (abdomen). Type-locality: Peru, Dept. Piura. Distr. - Peru, Chile. Type \& USNM*. Ref. - Fluke, 1950a:129, fig. 143 (male genitalia).

chilensis Sack, 1941:98 (1951:94), fig. 1 (abdomen). Type-localities: Peru, Ica, Laguna Victoria near Ica, Yaucatal near Ica, Huayuri; Chile. Types $0^{*}$ o $\mathrm{MD}$ ? N. SYN.

PlaUmanNi (Frey), 1946:168 (Microsphaerophoria). Type-locality: Brazil, Santa Catarina, Nova Teutônia. Distr. - same. Type ơ UZMH*. PUlchra Shannon, 1927a:25. Type-locality: Chile, Angol. Distr. - Chile (Coquimbo - Malleco). Type of USNM*. Ref. - Shannon \& Aubertin, 1933:132, figs. 24a' \& 24b' (head), 25b (abdomen); Fluke, 1942:24, fig. 51 (abdomen).

var. BIFASCIATA Enderlein, 1938:222. Type-locality: Chile, "Rango-Tal in den Cordilleren, Parral-Tal". Types o o ZMB.

var. NIGRIBUCCA Enderlein, 1938:222. Type-locality: Chile. "Rango-Tal, Bäder von Longavi, Parral, Cauquenes." Types of ZMB.

var. SHANNONI Enderlein, 1938:222. Type-locality: Chile, "Rango-Tal in den Cordilleren." Type $\$ \mathrm{ZMB}$.

QUADRICINCTA Enderlein, 1938:214. Type-locality: Colombia, Bogotá. Distr. - same. Types o ZMB.

REMIGIS (Fluke), 1942:9, fig. 15 (abdomen) (Epistrophe). Type-locality: Ecuador, Volcan Tungurahua, Minza Chica. Distr. - same. Type o AMNH*. Ref. - Fluke, 1950a:124, fig. 97 (male genitalia as altissimus).

RHINA Thompson (nom. nov, for nasuta Enderlein). Distr. - Paraguay. NOM. NOV.

nasuta Enderlein, 1938:214 (Chasmia; preocc. Bigot, 1884, 1888). Type-locality: Paraguay. Type $\%$ ZMB or DEI.

ROBINSONIANA Enderlein, 1940:664. Type-locality: Chile, Juan Fernández Islands, Masatierra. Distr. - same. Types ot o NRS.

ROBURORIS (Fluke), 1942:11, figs. 18 (abdomen), 19 (head) [Epistrophe (Fazia) ]. Type-locality: Ecuador, Bolivar, Hda. Talahua. Distr. Ecuador, Argentina (Tucumán). Type o AMNH*.

ROSTRATA (Bigot), 1884b:102 (Sphaerophoria). Type-locality: Mexico. Distr. - same. Types o OXF*. N. COMB.

Saussurir (Giglio-Tos), 1892d:2 (Syrphus). Type-locality: Mexico, Orizaba. Distr. - same. Type o UTOR*. N. COMB.

Schoenemanni Enderlein, 1938:225. Type-locality: Chile, Cauquenes. Distr. - same. Type o ZMB.

similis Curran, 1925c:350. Type-locality: Colombia, Vista Nieve. Distr. Colombia, Brazil (São Paulo, Santa Catarina), Argentina (Tucumán). Type \& CU*. Ref. - Fluke, 1942:23, fig. 27 (abdomen); 1950a:129, fig. 144 (male genitalia).

SPLENDENS (Thomson), 1869:501 (Syrphus). Type-locality: Galápagos. Distr. - same. Types on o NRS*.

Strigifacies (Enderlein), 1938:211 (Fazia). Type-locality: "Brazil". Distr. - same. Types or + ZMB. N. COMB.

SYCORAX (Hull), 1947b:239 (Rhinoprosopa). Type-locality: Costa Rica, La Suiza. Distr. - Costa Rica, Colombia, Venezuela. Type o USNM. SYRPHICA (Giglio-Tos), 1892d:2 (Sphuerophoria). Type-locality: Mexico, Orizaba. Distr. - same. Type o UTOR*. N. COMB, Ref. - GiglioTos, 1893:fig. 5 (abdomen), 5a (head).

surphica, Fluke, 1956:216, misspelling. 
Ref. - Hull 1949e:222, 270, figs. 149 (abdomen), 344 (wing). N. COMB.

TECTIFORMA Fluke, 1942:18, fig, 45 (abdomen). Type-locality: Ecuador, Imbabura Cuicocha, $3200 \mathrm{ft}$. Distr. - Ecuador. Type o AMNH*. Ref. - Fluke, 1950a:129, fig. 145 (male genitalia).

TELIGERA Fluke, 1942:18, fig. 44 (abdomen). Type-locality: Brazil, Santa Catarina, Nove Teutônia. Distr. - Ecuador, Brazil (Santa Catarina). Type o AMNH*.

TRANSVERSA (Hull), 1943f:32 (Sphaerophoria). Type-locality: U.S.A., Oregon, near Portland. Distr. - U.S.A. (Oregon south to Texas); Mexico. Type o. Hull.

TRIBINICINCTA Enderlein, 1938:224. Type-locality: Paraguay, San Bernardino. Distr. - same. Type $\sigma^{*} \mathrm{ZMB}$ or DEI.

TRIFASCIATA Enderlein, 1938:218. Type-locality: "Mexico". Distr. - same. Type o $\mathrm{ZMB}$.

TRILImbata (Bigot), 1888:253 (Sphaerophoria). Type-locality: Mexico. Distr. - same. Type o $\mathrm{OXF}^{*}$. N. COMB.

Tucumana Enderlein, 1938:220. Type-locality: Argentina, Prov. Tucumán. Distr. - same. Type o ZMB.

venusta Curran, 1927b:5. Type-locality: Virgin Islands, St. Croix, Blessing Estate, near Krause Lagoon in south central part of island. Distr. - U.S.A. (Florida); Puerto Rico, Virgin Islands: Type $\sigma^{*}$ AMNH*. Ref. - Fluke; 1942:24, fig. 38 (abdomen).

WILISToNI (Giglio-Tos), 1893:31, figs. 6 (head), 6a (abdomen) (Sphaerophoria). Type-locality: Mexico, Orizaba. Distr. - same. Types ơ ㅇ UTOR*. N. COMB.

\section{Genus SpHAEROPHORIA Lepeletier \& Serville}

SPHAEROPHORIA Lepeletier \& Serville, in Latreille et al., 1828:513. Typespecies, Musca scripta Linnaeus (Rondani, 1844:458).

Melithreptus Loew, 1840:577 (unjustified nom. nov.-for Sphaerophoria; preocc. Vieillot, 1816). Type-species, Musca scripta Linnaeus (aut.).

Melitropus Haliday, 1856:xxi (nom. nov. for Melithreptus Loew). Typespecies, Musca scripta Linnaeus (aut.).

CONTIGUA Macquart, 1847:78 (62). Type-locality: U.S.A., Pennsylvania, Philadelphia. Distr. - Canada (British Columbia to New Brunswick), south to U.S.A. (Florida) and Mexico. Type o OXF*.

cylindrica Say, 1824:pl. 11 (habitus) (Syrphus; preocc. Fabricius, 1781). Type-locality: U.S.A., Pennsilvania, Philadelphia, Type(s) ? lost.

fulvicauda Bigot, 1884b:104. Type-locality: Mexico. Type o OXF*.

PYRRHINA Bigot, : 1884b:101. Type-locality: U.S.A., California. Distr. U.S.A. (Idaho, California, Utah); Mexico [Wirth et al., 1965:570]. Type o OXF.

guttulata Hull, 1942h:20. Type-locality: U.S.A., Idaho, Black Foot. Type o Hull.

SULPHURIPES (Thomson), 1869:500 (Syrphus). Type-locality: California. Distr. - U.S.A. (Alaska, southeast to Nebraska), south to Mexico, Ecuador? (specimen in BM(NH)*. Type ơ $f$ NRS*.

pachypyga Bigot, 1884b:104. Type-locality: Mexico. Type o OXF*. 


\section{UNRECOGNIZED SYRPHINI}

\section{Genus Allograptina Enderlein}

ALLOGRAPTINA Enderlein, 1938:226. Type-species, octomaculata Enderlein (orig. des.).

octomaculata Enderlein, 1938:226. Type-locality: "Mexico". Distr. - same. Type o ZMB.

\section{Unplaced species}

AUROPUlveratus Macquart, 1842:159 (99) (Syrphus). Type-locality: "Chile". Type o MNHN (lost?) [label but no specimen in Macquart Collection at Paris - JRV].

BASALIS Walker, 1837:345 (Syrphus). Type-locality: Brazil, St. Paul Island. Type ? $\mathrm{BM}(\mathrm{NH})$.

basilaris, Williston, 1886:328, misspelling.

CHIQUITENSIS Macquart, 1850:458 (154) (Syrphus). Type-locality: Bolivia, Chiquitos. Type \& MNHN (lost?) [label but no specimen in Macquart Collection at Paris - JRV].

Colludens Walker, 1860:293 (Syrphus). Type-locality: "Mexico". Type of $\mathrm{BM}(\mathrm{NH})$.

Columbianus Rondani, 1863:11 (Syrphus). Type-locality: "Colombia". Type \& MIZUN?

DECEMMACUlatus Rondani, 1863:12 (Syrphus). Type-locality: "Chile". Type o MIZUN?

DELINEATUS Macquart, 1846:267 (139) (Syrphus). Type-locality: "Mexico". Type + MRHNB.

DISPAR Fabricius, 1794:309 (Syrphus). Type-locality: "America meridionalis". Types o $0^{*}$ KIEL (Destroyed - Zimsen, 1964:481).

ExCAvatus Rondani, 1850:359 (Syrphus). Type-locality: South America, Río Napo, [Ecuador]. Type o MCSNH?

FASCIVENTRIS Rondani, 1850:360 (Syrphus). Type-locality: South America, Río Napo, [Ecuador]. Type of MCSNM?

FLAVIGASTER Hull, 1944d:51 (Syrphus). Type-locality: "South America, Amazons". Type o $\mathrm{BM}(\mathrm{NH})$.

FUSCANIPENNIS Macquart, 1846:264 (136) (Syrphus). Type-locality: "Brazil". Type o OXF?

INANIS Fabricius, 1775:763 (Syrphus). Type-locality: "America". Type † KIEL.

exinanita Gmelin, 1790:2867 (Musca; nom. nov. for inanis Fabricius). NIGRITUS Fabricius, 1781:427 (Syrphus). Type-locality: "Jamaica". Type ? ?

jamaicensis Gmelin, 1790:2871 (Musca; nom. nov. for nigritus Fabricius).

PLURIMACUlatus Rondani, 1863:12 (Syrphus). Type-locality: "Amer. Meridion.". Type o MIZUN?

PUNCTATUS Macquart, 1842:152 (92) (Syrphus). Type-locality: "Chile". Type o MNHN [not found - JRV].

Radiatus Bigot, 1857:805 (Syrphus). Type-locality: Cuba. Type ? BM(NH)? [not at OXF - JRV].

RONDANII Goot, 1964:215 (Syrphus; nom. nov. for albitarsis Rondani). Distr. - Brazil, São Paulo, Ilha de São Sebastião. (S. Sebastiani Island).

albitarsis Rondani, 1850a:166, fig. 3 (wing) (Syrphus; preocc. Meigen, 
1822). Type-locality: Brazil, São Paulo, Ilha de São Sebastião (S. Sebastiani Island). Type UTOR.

vertebratus Rondani, 1863:10 (Syrphus). Type-locality: "Chile". Type o MIZUN?

\section{Tribe CHRYSOTOXINI}

\section{Genus Chrysotoxum Meigen}

Antiopa Meigen, 1800:32. Type-species, Musca bicincta Linnaeus (Coquillett, 1910a:508). Suppressed by I.C.Z.N., 1963:339.

CHRysotoxum Meigen, 1803:275. Type-species, Musca bicincta Linnaeus (Latreille,, 1810:443). Ref. - Shannon, 1926b (rev.).

\section{Subgenus PRIMOchrYsotoxum Shannon}

Chrysotoxum, subg. PRIMOCHRYSOTOXUM Shannon, 1926b:5. Type-species, Chrysotoxum ypsilon Williston (orig. des.).

A ZTEC Shannon, 1926b:8. Type-locality: Mexico, Chihuahua, Sierra Madre, $7300 \mathrm{ft}$. Distr. - same. Type or USNM.

INTEGRE Williston, 1887:16. Type-locality: U.S.A., Arizona. Distr. - Canada (British Columbia) to U.S.A. (Idaho), south to Mexico (N. Sonora). Type $q$ USNM. Ref. - Shannon, 1926b:11 (descriptive notes).

\section{Tribe PARAGINI}

Genus Paragus Latreille

PARAGUS Latreille, 1804:194. Type-species, Syrphus bicolor Fabricius (mon.).

\section{Subgenus Pandasyopthalmus Stuckenberg}

PANDASYOPTHALMUS Stuckenberg, 1954:100. Type-species, Paragus longiventris Loew (orig. des.).

Tibialis (Fallén), 1817:60 (Pipiza). Type-locality: "Vestrogothia; Scaniae". Distr. - Holaretic, ?Africa, ?Australia, ?Oriental; Mexico. Type ? ?

\section{Tribe MELANOSTOMATINI}

Ref. - Fluke, 1945 (rev., keys); Fluke, 1958 (male genitalia).

Genus Carposcalis Enderlein

CARPOSCALIS Enderlein, 1938:199. Type-species, Syrphus stegnus Say (orig. des.).

BERTRANDI (Austen), 1913:498 (Melanostoma). Type-locality: East Falkland Island. Distr. - Falkland Islands. Types ot of BM(NH)*. N. COMB. CHAETOPODA (Davidson), 1922:35 (Melanostoma). Type-locality: U.S.A., California, Alhambra. Distr. - U.S.A. (California, Colorado); Mexico (Mexico City). Type o* USNM. Ref. - Fluke, 1945:27, figs. 19 (head), 56 (front tibia of male); $1958: 273$, figs. $49 \& 50$ (male genitalia).

Chalconota (Philippi), 1865:747 (Syrphus). Type-locality: Chile, Santiago. 
Distr. - Colombia (Bogotá), Ecuador, Chile, Argentina (Río Negro, Tierra del Fuego). Type(s) ? MNHNS. Ref. - Fluke, 1945:15 (redescription), figs. 22 (head), 23 (antenna), 38 (abdomen); 1958:273, figs. 51 \& 52 (male genitalia).

ECUADORIENSIS (Fluke), 1945:16, figs. 25 (head), 59 (front tibia of male) [Melanostoma (Carposcalis)]. Type-locality: Ecuador, Imbabura, Cuicocha. Distr. — same. Type o $\mathrm{AMNH}^{*}$. Ref. - Fluke, 1958: 273, figs. $59 \& 60$ (male genitalia); Hull, 1949d:289, fig. 10c (head). ElWARDSI (Shannon \& Aubertin), 1933:133 (Melanostoma). Type-locality: Argentina, Bariloche. Distr. - same. Type o BM(NH)*. N. COMB.

FENESTRATA (Macquart), 1842:163 (103), tab. 17, fig. 6 (habitus), 6a (head) (Syrphus). Type-locality: "Chile". Distr. - Mexico (Durango, Guerrero), Brazil (Rio de Janeiro), Chile (Santiago to Magallanes), Paraguay, Argentina (Tucumán, Buenos Aires, Mendoza, Neuquén, Río Negro, Chubut, Tierra del Fuego). Types \& MNHN*. Ref. - Fluke, 1945:15 (descriptive notes), figs. 21 (head), 57 (male front tibia); Fluke, 1958:273, figs. $56 \& 64$ (male genitalia).

?producta Macquart, 1850:458 (154), tab. 14, fig. 13 (head) (Syrphus). Type-locality: Chile. Types o MNHN*.

INFLATifrons (Fluke), 1945:21, fig. 31 (head) (Melanostoma). Type-locality: Ecuador, Bolivar, Hda. Talahua. Distr. - same. Type of AMNH*. Ref. - Fluke, 1958:273, figs. 55 \& 63 (male genitalia). LUNDBladi Enderlein, 1940:665. Type-locality: Chile, Juan Fernández Islands, Masatierra: Distr. - same. Type o NRS. Ref. - Fluke. 1958:273, figs. $61 \& 62$ (male genitalia).

MEYERI (Fluke), 1945:17, fig. 24 (head) (Melanostoma). Type-locality: Mexico, Mexico City. Distr. - same. Type of AMNH*. N. COMB.

PUNCTUlata (Wulp), 1888:375 (Melanostoma). Type-locality: Argentina, Córdoba. Distr. - Ecuador, Peru, Brazil (São Paulo), Bolivia, Chile, Argentina (Tucumán, Catamarca, La Rioja, Jujuy, Mendoza, Buenos Aires, Río Negro, Neuquén). Types o $\sigma^{7}$ lost? Ref. - Fluke, 1945:14 (redescription), figs. 20 (head), 58 (front tibia of male); $1958: 275$, figs. $67 \& 68$ (male genitalia).

REYNOLDSI (Shannon \& Aubertin), 1933:134 (Melanostoma). Type-locality: Tierra del Fuego, Río Grande, Estancia Viamonte. Distr. - same. Types o o $\mathrm{BM}(\mathrm{NH}) *$. N. COMB.

SALtaNA Enderlein, 1938:200. Type-locality: Argentina, Salta, $2500 \mathrm{~m}$. Distr. - Ecuador, Argentina (Salta, Buenos Aires, Río Negro). Type o ZMB.

SCUTIGERA (Fluke), 1945:20, fig. 30 (head) (Melanostoma). Type-locality: Ecuador, Pichincha, Uyumbicho. Distr. - same. Type o AMNH*. N. COMB.

Stegna (Say), 1829:163 (Syrphus), Type-locality: "Mexico". Distr. Canada (British Columbia) to U.S.A. (Montana), south to Mexico (Durango, Guerrero, Vera Cruz). Type ? lost. Ref. - Fluke, $1945: 14$, figs. 18 (head), 55 (front tibia of male); 1958:275, figs. $73 \& 74$ (male genitalia).

WAL KERI (Lynch Arribálzaga), 1892b:117 (1892c:75) (Syrphus, nom. nov. for unicolor Walker). N. COMB.

unicolor Walker, 1837:344 (Syr)hus; preocc. Macquart, 1829). Typelocality: Argentina, Port Famine, Straits of Magellan. Type ? $\mathrm{BM}(\mathrm{NH}) *$. N. COMB. 


\section{Genus Melanostoma Schiner}

Melanostoma Schiner, 1860:213. Type-species, Musca mellina Linnaeus orig. des.).

BeUlum Giglio-Tos, 1892d:3. Type-locality: "Mexico". Distr. - same. Types o UTOR*. Ref. - Giglio-Tos, 1893: fig. 7 (abdomen), 7a (head).

Cruciatum Bigot, 1884b:81. Type-locality: "Mexico". Distr. - same. Type $\cdot \sigma^{\pi} \mathrm{OXF}^{*}$.

Dubiom (Zetterstedt), 1838:609 (Scaeva). Type-locality: Sweden, Torne Lappmark, Juckasjärvi. Distr. - Europe; U.S.A. (Utah, Colorado); Mexico? (Wirth, et al., 1965:575). Type 우 ZML (Andersson, 1970:238, lectotype designation).

MELlinum (Linnaeus), 1758:594 (Musca). Type-locality: "Europe". Distr. - Holarctic; Mexico? [Williston, 1891:11], Argentina? [Macquart, 1850:456 (152)]. Types \& LSL*. Ref. - Williston, 1887b:49 (redescription), pl. 3, fig. 9 (head).

PICTIPES Bigot, 1884b:78. Type-locality: U.S.A., California. Distr. - Canada (British Columbia east to Nova Scotia), south to Mexico (Mexico City, Guerrera). Types o $\mathrm{OXF}^{*}$.

pruinosa Bigot, 1884b:79. Type-locality: U.S.A., California. Type $q$ OXF.

\section{Genus RHYSOPS Williston}

RHYsops Williston, 1907:2. Type-species, Melanostoma rugosonasus Williston (Coquillett, 1910a:601, as rugonasus Williston).

Braziliana Curran, 1925b:252. Type-species, Melanostoma longicornis Williston (orig. des.) = longicornis Walker.

AGONIS (Walker), 1849:588 (Syrphus). Type-locality: Galápagos Islands. Distr. - same. Type o $\mathrm{BM}(\mathrm{NH}) *$ N. COMB.

ALTISSIMUS (Fluke), 1945:20, figs. 48 \& 49 (abdomen) (Melanostoma). Type-locality: Ecuador, Imbabura, Cuicocha. Distr. - Ecuador, Colombia. Type o AMNH*. Ref. — Fluke, 1958:277, figs. 95 \& 96 (male genitalia).

BERTHAE Lima, 1946:155, figs. 1 (head), 2 (abdomen), 3 (wing). Typelocality: Brazil, Rio de Janeiro. Distr. - same. Type o ENA.

BOLIVARIENSIS (Fluke), 1945:19, figs. 29 (head), 45 (abdomen) (Melanostoma). Type-locality: Ecuador, Bolivar, Hda. Talahua. Distr. same. Type o AMNH*. Ref. - Fluke, 1958:277, figs. 97 \& 98 (male genitalia).

BRowNI (Fluke), 1945:18, figs. 27 (antenna), 28 (head), 44 (abdomen) (Melanostoma). Type-locality: Ecuador, Bolivar, Hda. Talahua. Distr. - same. Type o AMNH*. Ref. - Fluke, 1958:277, figs. $107 \& 108$ (male genitalia).

CATABomba (Williston), 1891:12 (Melanostoma). Type-locality: Mexico, Guerrero, Omiltemi, $8000 \mathrm{ft}$.; Amula, $6000 \mathrm{ft}$. Distr. - same. Types $0^{*} \mathrm{BM}(\mathrm{NH}) *$ \& AMNH*. Ref. - Fluke, 1945:5 (redescription), fig. 2 (head).

COLUMBianUS (Enderlein), 1938:202 (Braziliana). Type-locality: Colombia, "Cordillera, tierra templada". Distr. - same. Types o" o ZMB.

CRENULATUS (Williston), 1891:12, pl. 1:5 (habitus), 5a $+\mathrm{b}$ (head) (Melanostoma). Type-locality: Mexico, Guerrero, Xucumanatlán, 7000 ft.; Omiltemi, 8000 ft. Distr. — same. Types $\sigma^{7}$ o $\mathrm{BM}(\mathrm{NH}) *$ \& AMNH*. Ref. - Fluke, 1945:5, fig. 3 (head). 
CURRANI Fluke, 1937:8, fig. 10 (abdomen). Type-locality: Brazil, Santa Catarina, Nova Teutônia. Distr. - same. Type ơ AMNH*. Ref. Fluke, 1945:9 (redescription), fig. 10 (head); 1958:277, figs. 101 \& 102 (male genitalia).

FASTIGATUS Fluke, 1945:4, figs. 4 (head), 37 (abdomen). Type-locality: Brazil, Santa Catarina, Nova Teutônia. Distr. - Brazil (Santa Catarina), Argentina (Tucumán). Type ơ AMNH*. Ref. — Fluke, 1958:277, figs. $99 \& 100$ (male genitalia).

FESTIVUS Fluke, 1845:10, figs. 13 (head), 42 (abdomen). Type-locality: Ecuador, Tungurahua, Baños, $1800 \mathrm{ft}$. Distr. - same. Type o AMNH*.

FUNEREUS Hull, 1949a:74. Type-locality: Brazil, Santa Catarina, Nova Teutônia. Distr. - same. Type ơ Hull.

LANEI (Fluke), 1936:61, figs. 4 (abdomen), 5 (head) (Melanostoma). Type-locality: Brazil, São Paulo. Distr. - Brazil (São Paulo, Santa Catarina), Argentina (Formosa, Salta, Tucumán). Type $\sigma^{7}$ AMNH*. Ref. - Fluke, 1945:26, fig. 14 (head); 1958:277, figs. $103 \& 104$ (male genitalia).

lachrymellea Hull, 1942b:10. Type-locality: Brazil, São Paulo. Type ơ Hull.

LINEATUS (Fluke), 1937:9, fig. 11 (abdomen) (Melanostoma). Type-locality: Brazil, Santa Catarina, Nova Teutônia. Distr. - same. Type AMNH*. Ref. - Fluke, 1945:7, fig. 7 (head); 1958:277, figs. 105 \& 106 (male genitalia).

LONGICORNIS (Walker), 1837:343 (Pipiza). Type-locality: "South America". Distr. - Guatemala, Colombia, Ecuador, Brazil (Mato Grosso, Santa Catarina), Paraguay. Type o $\operatorname{BM}(\mathrm{NH}) *$. Ref. - Fluke, 1945:3, fig. 1 (head); 1958:269, 279, figs. 4, 14, 133 (male genitalia). N. COMB.

longicornis Williston, 1888:263 (Melanostoma; preocc. Walker, 1837). Type-locality: Brazil, Mato Grosso, Chapada. Types o* + AMNH*. N. SYN.

vittithorax Hull, 1937a:176, fig. 9 (head) (Braziliana). Type-locality: Guatemala, Antigua. Type o USNM.

LOPESI Fluke, 1945:6, fig. 5 (head). Type-locality: Brazil, São Paulo, São José dos Campos. Distr. - same. Type o AMNH*.

LUCUlentus (Fluke), 1945:17, figs. 26 (head), 46 (abdomen) (Melanostoma). Type-locality: Ecuador, Tungurahua, Baños. Distr. - same. Type o* AMNH*. N. COMB.

Maculatus (Walker), 1852:220 (Paragus). Type-locality: "Brazil". Distr. Brazil (Santa Catarina), Argentina (Tucumán). Type o $\mathrm{BM}(\mathrm{NH}) *$. Ref. - Fluke, 1958:279, figs. $109 \& 110$ (male genitalia). N. COMB.

minuscula Fluke, 1945:11, figs. 16 (head), 17 (antenna), 47 (abdomen). Type-locality: Brazil, Santa Catarina; Nova Teutônia. Type o AMNH*. N. SYN.

discretum Frey, 1946:157 (Melanostoma). Type-locality: Brazil, Santa Catarina, Nova Teutônia. Type o UZMH*. N. SYN.

MELANocera (Williston), 1891:13 (Melanostoma). Type-locality: Costa Rica, Río Sucio. Distr. - same. Types $q \mathrm{BM}(\mathrm{NH})$.

NEOTRopicus (Curran), 1937:4 (Melanostoma). Type-locality: Brazil, São Paulo. Distr. - Brazil. (São Paulo, Santa Catarina), Argentina (Misiones). Type o AMNH*. Ref. - Fluke, 1958:279, figs. 117 \& 118 (male genitalia).

columella Fluke, 1945:8, figs. 9 (head), 39 \& 40 (abdomen). Typelocality: Brazil, Santa Catarina, Nova Teutônia. Type ơ AMNH* 
NIGRANS Fluke, 1945:8, fig. 8 (head). Type-locality: Brazil, Santa Catarina, Nova Teutônia. Distr." - Brazil (São Paulo, Santa Catarina). Type \& AMNH*. Ref. - Fluke, 1958:279, figs. 111 \& 112 (male genitalia).

OPACUS Fluke, 1945:10, fig. 15 (head). Type-locality: Ecuador, Chimborazo, Urbina, Cerro Chimborazo. Distr. - same. Type o AMNH*. Ref. - Fluke, 1958:279, figs. $115 \& 116$ (male genitalia).

PERUVIANus (Shannon), 1927a:7 (Braziliana). Type-locality: Peru, Río Charape. Distr. - same. Type o USNM.

Pollinosus Hull, 1942b:10. Type-locality: Brazil, São Paulo. Distr. -Brazil (São Paulo), Argentina (Tucumán). Type q Hull. Ref. Fluke, 1945:26, fig. 11 (head); 1958:279, figs. 113 \& 114 (male genitalia).

PRAEUSTUS (Loew), 1866a:155 (1872b:29) (Syrphus). Type-locality: Cuba. Distr. - same. Type of MCZ*. N. COMB.

QUadrimaculatus Hull, 1944c:28. Type-locality: Cuba, Wright. Distr. Cuba, Dominican Republic. Type o MCZ*. Ref. - Fluke, 1945:9 (redescription), figs. 12 (head), 41 (abdomen).

REX (Fluke), 1945:21, figs. 32 (head), 50 (abdomen) (Melanostoma). Type-locality: Ecuador, Bolivar, Hda. Talahua. Distr. - same. Type ot AMNH*. Ref. - Fluke, 1958:277, figs. 93 \& 94 (male genitalia).

RUgosonasus (Williston), 1891:13 (Melanostoma). Type-locality: Mexico, Guerrero, Omiltemi, $8000 \mathrm{ft}$. Distr. - Mexico (Mexico City, Guerrero). Type o $\mathrm{BM}(\mathrm{NH}) *$. Ref. - Fluke, 1945:7 (redescription), fig. 6 (head); 1958:269, 279, figs. $6,15 \& 130$ (male genitalia).

rugonasus, Aldrich, 1905:361; Williston, 1907:2, Coquillett, 1910a:601, misspellings.

SCITULUS (Williston), 1888:264 (Melanostoma). Type-locality: Brazil, Mato Grosso, Chapada. Distr. - same. Types o AMNH*. Ref. - Fluke, 1945:7 (redescription), figs. 43 (abdomen), 54 (wing).

THIEMEI (Enderlein), 1938:201 (Braziliana). Type-locality: Colombia. "Cordillera, tierra templada". Types o $o^{*}$ ZMB.

TROPICUS (Curran), 1937:3 (Melanostoma). Type-locality: Brazil, São Pau Campos do Jordão. Distr. - Ecuador, Brazil (São Paulo), Argentỉna (Tucumán). Type ơ AMNH. N. COMB.

\section{Genus XanthandRus Verrall}

XANTHANDRUS Verrall, 1901:316. Type-species, Musca comptus Harris (Coquillett, 1910a:620). Ref. - Fluke, 1937 (key).

Biguttatus Hull, 1945b:73. Type-locality: Paraguay, Molino-Cué. Distr. same. Type o MCZ*.

BUCEPHALUS (Wiedemann), 1830:126 (Syrphus), Type-locality: "Brazil". Distr. - Peru, Brazil (São Paulo). Type ơ ZMB. Ref. - Fluke, 1958:279, figs. $119 \& 120$ (male genitalia).

cubanus Fluke, 1936:63, figs. 6 (abdomen), 7 (head). Type-locality: Cuba, Sierra del Cobra, Loma del Gato, 2600-3325 ft. Distr. - Cuba, Puerto Rico. Type o AMNH*.

nigripes Loew, 1866a:155 (1872b:29). (Syrphus, preocc. Meigen, 1822). Type-locality: "Cuba". Type o MCZ*. N. SYN.

loewi Gott, 1964:215 (Syrphus; unjustified nom. nov. for nigripes Loew). N. SYN.

Flavomaculatus Shannon, 1927a:6. Type-locality: Bolivia, Beni, Rurrena- 
baque. Distr. - same. Type or USNM. Ref. - Fluke, 1937:5, fig. $?$ (abdomen).

MELLINOIDES (Macquart), 1846:265 (137) (Syrphus). Type-locality: "Colombia". Distr. - Mexico, Colombia, Venezuela. Type ơ OXF* N. COMB.

euceratus Bigot, 1884b:82 (Melanostoma?). Type-locality: Colombia, Bogotá. Type ơ OXF*. N. SYN.

Mexicanus Curran, 1930f:9. Type-locality: Mexico, Yucatán, Chichén Itzá. Distr. - U.S.A. (Texas); Mexico (Chiapas, Yucatán), Costa Rica. Type o AMNH*.

NITIDULuS Fluke, 1937:7, fig, 8 (abdomen). Type-locality: Brazil, Santa Catarina, Nova Teutônia. Distr. - Brazil (Santa Catarina), Argentina (Jujuy, Salta). Type o AMNH*. Ref. - Fluke, 1958:279, figs. $121 \& 122$ (male genitalia).

PALliatus (Fluke), $1945: 22$, figs. 33 (head), 35 (antenna), 51 \& 52 (abdomen) (Melanostoma). Type-locality: Ecuador, Tungurahua, Minza Chica. Distr. - same. Type o AMNH*. N. COMB.

PlaUmannI Fluke, 1937:8, fig. 4 (abdomen). Type-locality: Brazil, Santa Catarina, Nova Teutônia. Distr. - same. Type q AMNH*.

QUADRINotatus (Bigot), 1884b:77 (Melanostoma?). Type-locailty: "Mexico". Distr. - same. Type o OXF*. N. COMB.

SIMPleX (Loew), 1861:40 (Syrphus). Type-locality: "Cuba". Distr. same. Type o MCZ*. N. COMB.

SMITHI (Goot), 1964:215 (Syrphus; nom. nov. for albomaculatus Smith). Distr. - Galápagos Islands.

albomaculatus Smith, 1877:84 (Syrphus; preocc. Macquart, 1842). Type-locality: Ecuador, Galápagos Islands, Charles Island. Type ? $\mathrm{BM}(\mathrm{NH})$ [not found - JRV].

\section{Genus Talahua Fluke}

Melanostoma, subg. TALAHUA Fluke, 1945:22. Type-species, fervidum Fluke (orig. des.).

FERvidA (Fluke), 1945:23, figs. 34 (head), 35 (male genitalia), 53 (abdomen) (Melanostoma). Type-locality: Ecuador, Bolivar, Hda. Talahua. Distr. - same. Type o AMNH*. Ref. - Fluke, 1958:279, fig. 123 (male genitalia).

\section{Genus Tuberculanostoma Fluke}

TUberculanostoma Fluke, 1943:425. Type-species, antennatum Fluke (orig. des.). Ref. - Fluke, 1943 (key).

ANTENNATUM Fluke, 1943:426, figs, 1 \& 2 (head), 3 (antenna). Typelocality: Ecuador, Bolivar, Hda. Talahua, $3100 \mathrm{ft}$. Distr. - Ecuador. Type o AMNH*. Ref. - Fluke, 1958:279, figs. 124 \& 125 (male genitalia).

BRowNI Fluke, 1943:429, figs. 7 (head), 8 (front femur and tibia). Typelocality: Ecuador, Chimborazo, Cerro Chimborazo, Urbina, $3650 \mathrm{ft}$. Distr. - Ecuador. Type o AMNH*.

CILIUM Fluke, 1943:428, figs. 4 \& 5 (head). Type-locality: Ecuador, Tungurahua, Volcán Tungurahua, Minza Chica, $3200 \mathrm{ft}$. Distr. - same. Type of AMNH*.

PECTINIS Fluke, 1943:430, figs. 6 (head), 9 (front femur). Type-locality: Ecuador, Bolivar, Hda. Talahua, $3100 \mathrm{ft}$. Distr. - same. Type of AMNH*. 


\section{Genus LeUcopodella Hull}

LeUCOPODELla Hull, 1949e:94. Typespecies, Baccha lanei Curran (orig. des.).

Xestoprosopa Hull, 1949e:94. Type-species, Baccha delicatula Hull (orig. des.). N. SYN.

ASthenia (Hull), 1948a:4 (Baccha). Type-locality: Brazil Santa Catarina, Nova Teutônia. Distr. - Ecuador, Brazil (São Paulo, Santa Catarina). Type o AMNH*. Ref. - Hull, 1943j:65, fig. 2 (alula); 1949e:228, figs. $170 \& 175$ (abdomen).

BALBOA (Hull), 1947b:230 (Baccha). Type-locality: Costa Rica, La Suiza. Distr. - same. Type o USNM. Ref. - Hull, 1949e:228, figs. 172 \& 174 (abdomen).

Bella (Hull), 1947a:401 (Baccha). Type-locality: Costa Rica, La Suiza. Distr. - same. Type o USNM. Ref. - Hull, 1949e:228, fig. 173 (abdomen).

BIGOTI (Austen), 1893:147 (Baccha; nom. nov. for apicalis Bigot). Distr. Panama, Brazil. Ref. - Hull, 1949e:228, 264, figs. 171 (abdomen), 322 (wing).

apicalis Bigot, 1883b:334 (Baccha; preocc. Loew, 1858). Type-locality: "Brazil". Type o BM(NH).

bigotii, Hull, 1949e:104, 287, misspelling.

BIPUNCTIPENNIS (Hull), 1942a:99 (Baccha). Type-locality: Paraguay, Bella Vista. Distr. - same. Type o. MCZ. Ref. - Hull, 1949e:204, 264, figs. 62 (abdomen), 324 (wing).

BOABDilla (Hull), 1947a:403 (Baccha). Type-locality: Paraguay, Alto Paraná, Hohenau. Distr. - same. Type o USNM. Ref. - Hull, 1949e:228, 264, figs. 177 (abdomen), 320 (wing).

BOAdICEA (Hull), 19431:72 (Baccha). Type-locality: Ecuador, Pinas, $1506 \mathrm{~m}$. Distr. - same. Type o AMNH*. Ref. - Hull, 1949e:202, fig. 55 (abdomen).

CARMElita (HuIl), 1948a:9 (Baccha). Type-locality: Haiti, La Visite; vic. La Selle Range, 5-7000 ft. Distr. - same. Type o MCZ.

DELICATUla (Full), 1943j:78, fig. 72 (abdomen) (Baccha). Type-locality: Ecuador, Tungurahua, Baños. Distr. - same. Type $0^{*}$ Hull. Ref. - Hull, 1949e:206, 264, figs. 72 (abdomen), 327 (wing). N. COMB.

ESTRELITA (Hull), 1948a:1 (Baccha). Type-locality: Cuba, Trinidad Mts, Buenos Aires. Distr. - Cuba; (Argentina record in Fluke, 1956: 245, is an error). Type o $\mathrm{MCZ}$.

GOWDEYI (Curran), 1926c:102 (Baccha). Type-locality: Jamaica, Cinchona. Distr. - same. Type o BM(NH). Ref. - Hull, 1949e:206, 256, figs. 73 (abdomen), 292 (wing).

GRACILIS (Williston), 1891:34 (Baccha). Type-locality: Mexico, Vera Cruz, Orizaioa; Guerrero, Omiltemi, 8000 ft. Distr. - Mexico (Sinaloa, Durango, Vera Cruz, Guerrero, Chiapas). 'Types ơ $\$ \mathrm{BM}(\mathrm{NH}) *$. Ref. - Hull, 1949e:228, fig. 176 (abdomen).

INCOMPTA (Austen), 1893:147, pl. 4:13 (habitus) (Baccha). Type-locality: Brazil, Region of Amazon. Distr. - Puerto Rico, Honduras, Guyana, Argentina (Jujuy, Tucumán). Types o $\sigma^{*} \mathrm{BM}(\mathrm{NH})$. Ref. Hull, 1949e:264, fig. 323 (wing).

LANEI (Curran), 1936b:16 (Baccha). Type-locality: Brazil, São Paulo, Campos do Jordão. Distr. - Trinidad, Brazil (São Paulo, Santa Catarina). Type o AMNH*. Ref. - Carrera, 1940:66 (description of female), fig. 1 (habitus); Hull, 1949e:198, 264, figs. 31 (abdomen), 321 (wing); 1949d:289, fig. 10e (head). 
MARMORATA (Bigot), 1883d:333 (Baccha). Type-locality: "Mexico". Distr. U.S.A. (Arizona), Mexico (Durango). Type o BM(NH). Ref. Giglio-'Tos, 1893:fig. 19 (head).

OLGA (Hull), 1942a:95 (Baccha). Type-locality: Panama, Chilibre, Distr. same. Type o MCZ. Ref. - Hull, 1949e:204, 264, figs. 67 (abdomen), 325 (wing).

RUBIDA (Williston), 1891:34, pl. 1:8 (habitus), 8a+b (heads) (Baccha). Type-locality: Mexico, Guerrero, Omiltemi, 8000 ft.; Sierra de las Aguas Escondidas, $9500 \mathrm{ft}$. Distr. - same. Types o $+\mathrm{BM}(\mathrm{NH})$ \& AMNH*. Ref. - Hull, 1949e:200, 238, 264, figs. 44 (abdomen), 317 (abdomen), 326 (wing).

ZENILlA (Hull), 1943j:77 (Baccha). Type-locality: Ecuador, Bolivar, Hda. Talahua, 3100 m. Distr. - same . Type o AMNH*. Ref. - Hull, 1949e:238, 256, figs. 218 (abdomen), 291 (wing).

\section{Unplaced species of MeLANOSTOMINI}

COLUMBIANUM Enderlein, 1938:198 (Melanostoma). Type-locality: Colombia, "Cordilleren, tierra fria". Type of ZMB?

CRASSICALX Enderlein, 1938:197 (Pachysphyria). Type-locality: Brazil, Santa Catarina, Hochland. Type $\sigma^{*}$ o ZMB?

Craneocincta (Bigot), 1885a:251 (Melanostoma). Type-locality: "Mexico". Type $\sigma^{\circ}$ OXF?

LIMBATum Enderlein, 1938:198 (Melanostoma). Type-locality: Argentina, Prov. Tucumán. Types o $\sigma^{\pi}$ ZMB?

LONGIGENA Enderlein, 1912:102 (Melanostoma). Type-locality: Tierra del Fuego, Ushuaia. Type o ZMB?

\section{Tribe TOXOMERINI}

\section{Genus Toxomerus Macquart}

TOXomerus Macquart, 1855:112 (1855:92). Type-species, notatus Macquart (orig. des.) = geminatus (Say).

Mesogramma Loew, 1866a:157 (1872b:31) (preocc. Stephens, 1850). Typespecies, parvula Loew (Williston, 1887b:98).

Mesograpta Loew, 1872:114 (1872b:290) (nom. nov. for Mesogramma Loew). Type-species, parvula Loew (aut.).

Mitrosphen Enderlein, 1938:227. Type-species, Syrphus tibicen Wiedemann (orig. des.).

Antiops Enderlein, 1938:228. Type-species, Mesograpta ? saphiridiceps Bigot (orig. des.).

Hybobathus Enderlein, 1938:233. Type-species, quadrilineatus Enderlein (orig. des.).

Hypobathus, Fluke, 1956:217, misspelling.

Ref. - Hull, 1943i (key).

AEolus (Hull), 1942d:47 (M€sogramma). Type-locality: Cuba, Soledad, Atkins Institute, Harvard Gardens. Distr. - same. Type o Hull. N. COMB.

ALPHABETICUS (Hull), 1942a:105 (Mesogramma). Type-locality: Mexico, Vera Cruz, Jalapa. Distr. - same. Type o AMNH*. Ref. - Hull, 1943i:35, fig. 50 (abdomen). N. COMB.

var. Flsiropocula (Hull), 1948b:9 (Mesogramma). Type-locality: Venezuela, San Esteban. Distr. - same. Type o Hull. N. COMB. 
ANNULIFER. (Bigot), 1884b:84 (Melanostoma?). Type-locality: "Mexico". Distr. - same. Type o OXF*. N. COMB.

ANTHRAX (Schiner), 1868:350 (Mesogramma). Type-locality: "South America". Distr. - Colombia, Guyana, Ecuador, Peru. Types ơ $q$ VMNH*.

var. FlaMmARIUS (Hull), 1943i:27 (Mesogramma). Type-locality: Honduras, Puerto Castilla. Distr. - same. Type o Hull. N. COMB.

ANTIOPA (Hull), 1951a:3 (Mesogramma). Type-locality: Ecuador, Chimborazo, Urbina. Distr. - Ecuador. Type o AMNH*. N. COMB.

Aquilinus Sack, 1941:100 (1951:96), fig. 2 (abdomen). Type-locality: Peru, Bolivia. Distr. - Panama, Peru, Brazil, Bolivia, Argentina. Types o $q$ MD? Ref. - Hull, 1943i:35, fig. 41 (abdomen).

anchoratus, auctorum.

IRCIFER (Loew), 1866a:160 (1872b:34) (Mesograpta). Type-locality: "Cuba" Distr. - Cuba, Jamaica, Haiti, Puerto Rico, Dominica, Ecuador, Peru. Types o + MCZ*. Ref. - Hull, 1943i:33, figs. 67, 68 (abdo$m \in n$ ).

ARCTURus (Hull), 1943i:18 (Mesogramma). Type-locality: Brazil, Santa Catarina, Nova Teutônia. Distr. - same. Type o AMNH*. N. COMB.

AURUlentus (Williston), 1887b:102 (Mesograpta). Type-locality: Cuba, San Domingo. Distr. - Cuba, Costa Rica. Types o \& USNM*. Ref. Hull, 1943i:29, fig. 12 (abdomen).

AZURLiNeUS (Hull), 1941i:433 (Mesogramma). Type-locality: British Honduras, El Cayo. Distr. - British Honduras. Type o USNM*. Ref. - Hull, 1943i:29, fig. 10 (abdomen). N. COMB.

BARBULUS (Walker), 1852:238 (Syrphus). Type-locality: "Brazil". Distr same. Types o $\mathrm{BMMH}^{*}$. N. COMB.

BASILARIS (Wiedemann), 1830:143 (Syrphus). Type-locality: "Brazil". Distr. - U.S.A. (Texas), West Indies, Greater Antilles, Lesser Antilles, Mexico, south to Chile and Argentina. Type o VMNH*. soror Schiner, 1868:350 (Mesogramma). Type-locality: "America". Types o $0^{\pi}$ VMNH.

variabilis Wulp, 1883:6, pl. 1, fig. 9 (abdomen) (Mesograpta). Typelocality: "Guadeloupe". Types o MRHNB*. \& MA*.

flavocuneus Hull, 1940b:433 (Mesogramma; as basilaris var.). Typelocality: Honduras, Salada, nr. Ceiba: Type o Hull. Ref. - Hull, 1943:37, fig. 53 (abdomen). N. SYN.

neotropicus Hull, 1943i:36, fig. 54 (abdomen) (also p. 14, as neotropica, in key) (Mesogramma). N. SYN.

var. BIFIDUS (Hull), 1943i:26 (Mesogramma). Type-locality: Ecuador, Baños. Distr. - same. Type or Hull? N. COMB.

BIDENTATUS (Giglio-Tos), 1893:49, pl. II, fig. 12 (abdomen) (Mesogramma). Type-locality: Mexico, Acaguizotla, Chilpancingo, Tepetlapa, Medellin nr. Vera Cruz, Teapa, Orizaba and Tampico. Distr. - Mexico, Guatemala, Costa Rica, Panama. Types o $o^{*}$ UTOR*.

BIPUNCTATUS (Hull), 1943i:23 (Mesogramma). Type-locality: Brazil, Santa Catarina, Nova Teutônia. Distr. - same. Type o AMNH*. N. COMB.

BISTRIGA (Bigot), 1884b:110 (Mesograpta?). Type-locality: "Mexico". Distr. - Mexico, Guatemala, El Salvador, Nicaragua, Panama. Type $q$ OXF*. Ref. - Hull, 1943i:39, fig. 71 (abdomen).

bistrigata Aldrich, 1905:370, Fluke, 1956:219; misspellings. 
BOSCII (Macquart), 1842:160 (100), pl. 17, fig. 2 (Syrphus). Type-locality: "Carolina". Distr. - Southeastern U.S.A.; Mexico, Greater and Lesser Antilles, Type + MNHN*. Ref. - Hull, 1943i:38, fig. 65 $(a b d o m \in n)$.

BREvifacies (Hull), 1943i:20 (Mesogramma). Type-locality: Ecuador, Baños, Runtum Trail. Distr. - Ecuador, Colombia (Popayán). Type o AMNH*. N. COMB.

brevifacia Hull, 1943i:14 (Mesogramma). Incorrect original spelling. CALCEOLATUS (Macquart), 1842:151 (91), pl. 16, fig. 1 (head) (Syrphus). Type-locality: "Chile". Distr. - Chile (Coquimbo - Malleco), Argentina (Neuquén). Types ơ q MNHIN*. Ref. - Shannon \& Aubertin, 1933:138, fig. 26a (abdomen). N. COMB.

Camilla (Hull), 1951a:20 (Mesogramma). Type-locality: Brazil, Santa Catarina, Nova Teutônia. Distr. - same. Type o* AMNH*. N. COMB.

Centaureus (Hull), 1951a:18 (Mesogramma). Type-locality: Colombia, Cali Dist., W. Cordillera. Distr. - same. Type o AMNH*. N. COMB.

crliatus (Giglio-Tos), 1892d:3 (Mesogramma). Type-locality: Mexico, Tuxpango. Distr. - Mexico, Peru. Types ơ o UTOR*. Ref. - GiglioTos, 1893:60, pl. II, fig. 16 (abdomen), 16a (head), 16b (leg).

Circumdatus (Bigot), 1884b:107 (Mesograpta?). Type-locality: "Mexico". Distr. - same. Types of o OXF*. Ref. - Hull, 1943i:41, fig. 74 (abdomen).

ClarACUNEus (Hull), 1942a:106 (Mesogramma). Type-locality: Écuador, Río Margajitas, Río Pastaza. Distr. - same. Type o AMNH*. Ref. - Hull, 1943i:37, fig. 49 (abdomen). N. COMB.

CLARUS (Curran), 1930b:11 (Mesogramma). Type-locality: Panama, Canal Zone, New Culebra, France Field, Barro Colorado Island. Distr. Panama (Canal Zone), Venezuela (San Esteban). Type ơ AMNH*. Ref. - Hull, 1943i:39, fig. 62 (abdomen). N. COMB.

comma (Giglio-Tos), 1892d:4 (Mesogramma). Type-locality: "Mexico". Distr. - same. Types o UTOR*.

CONFUSUS (Schiner), 1868:349 (Mesogramma). Type-locality: "South America" - Venezuela; Brazil, Rio de Janeiro. Distr. - Mexico, Guatemala, Nicaragua, Costa Rica, Panama, Trinidad, Tobago, Ecuador, Venezuela Brazil, Argentina. Types o + VMNH*. Ref. Hull, 1943i:31, fig. 20 (abdomen).

maculipes Bigot, 1884b:113 (Mesograpta?). Type-locality: "Brazil". Type o OXF*.

Costalis (Wiedemann), 1830:140 (Syrphus). Type-locality: "Surinam". Distr. - Surinam. Type ơ SMF*. N. COMB.

CRCCKERI (Curran), 1934c:154 (Mesogramma). Type-locality: Galápagos Islands, Charles I. Distr. - Galápagos Islands. Type o CAS*. Ref. - Hull, 1943i:33, fig. 36 (abdomen). N. COMB.

cocheri Fluke, 1956:220, misspelled.

CRoEsus (Hull), 1940a:248 (Mesogramma). 'Type-locality: Brazil, São Paulo. Distr. - same. Type o Hull. Ref, - Hull, 1943i:31, fig. 14 (abdomen). N. COMB.

CYRILluS (Hul1), 1951a:9 (Mesogramma). Type-locality: Ecuador, Macas, Río Upano. Distr. - same. Type o AMNH*, N. COMB.

DTFFicilis (Curran), 1930b:6, fig. 2 (male genitalia) (Mesogramma). Typelocality: Puerto Rico, Coamo Springs. Distr. - Puerto Rico, Guya- 
na. Type o AMNH*. Ref. - Hull, 1943i:28, fig. 11 (abdomen). N. COMB.

Diversus (Giglio-Tos), 1892d:3 (Mesogramma). Type-locality: "Mexico". Distr. - same. Types \& UTOR*. Ref. - Giglio-Tos, 1893:49, pl. II, fig. 13 (abdomen).

Duplicatus (Wiedemann), 1830:142 (Syrphus). Type-locality: Uruguay, Montevideo. Distr. - Greater Antilles, Lesser Antilles, Mexico, south to Chile and Argentina. Types of o VMNH*. Ref. - Hull, 1943i:33, fig. 33 (abdomen).

ochrogaster Thomson, 1869:494 (Syrphus). Type-locality: Argentina, Buenos Aires, Type o* NRS*.

ECTyPus (Say), 1829:165 (Syrphus). Type-locality: "Mexico". Distr. same. Types ? lost.

ECUAdoreus (Hull), 1943i:19 (Mesogramma). Type-locality: Ecuador, Pichincha. Distr. - Ecuador. Type o AMNH*. N. COMB.

ELISA (Hull), 1951a:5 (Mesogramma). Type-locality: Brazil, São Paulo. Distr. :- same. Type of AMNH*. N. COMB.

ELoNGatus (Hull), 1941f:46 (Mesogramma). Type-locality: Colombia, Muzo, Dept. Boyaca. Distr. - Mexico, Colombia, Ecuador. Types o Hull, MCZ*. Ref. - Hull, 1943i:35, fig. 42 (abdomen). N. COMB.

interruptus Enderlein, 1938:227 (Mitrosphen; preocc. Philippi, 1865). Type-locality: Colombia, Cordillera; Mexico, Jacuapam. Types ơ $q$ ZMB. N. SYN.

EURydice (Hull), 1951a:17 (Mesogramma). Type-locality: Ecuador, San Francisco, Rio Pastaza. Distr. - same. Type o AMNH*. N. COMB.

eXtrapolatus (Hull), 1943i:22, fig. 24 (abdomen) (Mesogramma). Typelocality: Cuba, Soledad. Distr. - same. Type ơ Hull*. N. COMB.

FLAVIPLURUS (Hall), 1927:239, fig. (abdomen) (Mesogramma). Type-locality: Guatemala, Puerto Barrios. Distr. - Guatemala; Brazil (Belém); Trinidad. Type o OhSU. N. COMB.

flavipleura Hull, 1943i:15, 28, misspelled.

FLAVuS (Hull), 1941i:432 (Mesogramma). Type-locality: Colombia, Restrepo. Distr. - Colombia, Ecuador, Peru, Bolivia. Type of USNM. Ref, - Hull, 1943i:35, fig. 44 (abdomen). N. COMB.

FLoRALIS (Fabricius), 1798:563 (Scaeva). Type-locality: "Cajenne". Distr. U.S.A., Florida; Greater Antilles; Lesser Antilles; Mexico, south to Chile and Argentina. Type ? Kiel (lost ? Zimsen, 1964:481). Ref. - Hull, 1943i:35, figs. 37-40 (abdomen). 'N. COMB.

flammineus Hull, 1941h:312 (IMesogramma). Type-locality: Brazil, São Páulo, Severínia. Type ơ Hull. Ref. - Hull, 1943i:6, 34 (as var.).

F̂̀veŚTus (Doesburg), 1966:65, fig. 56 (habitus) (Mesograpta). Typelocality: Surinam, Zanderij. Distr. - Surinam, Brazil (Pará). Type o Doesburg. N. COMB.

GEMINI (Hull), 1941h:312 (Mesogramma). Type-locality: Brazil, São Paulo. Distr. - same. Type o Hull, MZUSP. Ref. - Hull, 1943i:33, fig. 25 (abdomen). N. COMB.

GUTTIFER (Hull), 1943k:39 (Mesogramma). Type-locality: Guatemala, Guatemala City. Distr. - Guatemala, Guyana. Type $q$ USNM*. Ref. Hull, 1943i:45, fig. 61 (abdomen). N. COMB.

HARLEQUINUS (Hull), 1951b:69 (Mesogramma). Type-locality: Brazil, São Paulo. Distr. - same. Type \& MZUSP. N. COMB.

HaRAldicus (Bigot), 1884b:109 (Mesograpta?). Type-locality: "Mexico". 
Distr. - same. Types ㅇ OXF*. Ref. - Hull, 1943i:47, fig. 73 (abdomen).

HIEROGLYPHICUS (Schiner), 1868:348 (Mesogramma). Type-locality: "South America". Distr. - Colombia (Magdalena, Valle del Cauca, Cauca), Ecuador. Type of VMNH*. Ref. - Hull, 1943i:31, fig. 19 (abdomen).

HUlli Sedman, (nom. nov. for ornatus Hull). Distr. - Paraguay. NOM. NOV.

ornatus Hull, 1943f:36 (MEsogramma, preocc. Sack 1941). Type-locality: Paraguay, Villarica. Type $\sigma^{*} \operatorname{AM}(\mathrm{NH})$.

IDALIUS (Hull), 1951a:11 (Mesograma). Type-locality: Ecuador, Oriente, Puyo. Distr. - same. Type o AMNH*. N. COMB.

subsp. LEDE Hull, 1951a:12 (Mesogramma). Type-locality: Ecuador, Oriente, Puyo. Type o AMNH*. N. COMB.

IMPERIALIS (Curran), 1926c:103 (Mesogramma). Type-locality: Jamaica, Blue Castle. Distr. - same. Type $+\mathrm{BM}(\mathrm{NH})$. Ref. - Hull, 1943i: 35, fig. 45 (abdomen). IN. COMB.

INCAICuS Sack, 1941:101 [1951:97, fig. 3 (abdomen)]. Type-locality: Peru, Querobamba, Lago Titicaca; Bolivia. Distr. - same. Types of $q$ MD?

INSIGNIS (Schiner), 1868:347, fig. 7 (habitus), 7 a-b (head) (Mesogramma). Type-locality: "South America". Distr. - same. Types $\sigma^{\pi}+$ VMNH*.

INTERMEDIUS (Hull), 1949a:77 (Mesogramma). Type-locality: Peru, Pucallpa. Distr. - same. Type o Hull. N、 COMB.

JAGUARINUS (Bigot), 1884b:112 (Mesograpta?). Type-locality: "Brazil". Distr. - same. Type o OXF*.

LACINIOSUS (Loew), 1866a:159 (1872b:33) (Mesograpta). Type-locality: "Cuba". Distr. - Greater Antilles, Lesser Antilles, Mexico south to Brazil and Argentina. Type o MCZ*. Ref. - Hull, 1943i:35, fig. 47 (abdomen).

LACRYMOSUS (Bigot), 1884b:107 (Mesograpta?). Type-locality: "Brazil; Mexico". Distr. - same. Types ơ \& OXF*. Ref. — Hull, 1943i:31, fig. 23 (abdomen).

lacrimosus Kertész, 1910:147, emendation.

lachrymosus, many authors, emendation.

LAENUS (Walker), 1852:241. (Syrphus). Type-locality: "Brazil". Distr. same. Type o BMNH. Ref. - Hull, 1943i:41, fig. 79 (abdomen). N. COMB.

laenas Hull, 1943i:16, 40, misspelled.

LANEI (Hull), 1942c:74 (Mesogramma). Type-locality: Brazil, Rio de Janeiro, Tinguá. Distr. - same. Type \& Hull. N. COMB.

LIMAE (Brèthes), 1920:30 (1937:VII), (Mesograpta). Type-locality: Peru. Lima. Distr. - same. Type ? MACN. N. COMB.

LimBuS (Enderlein), 1938:229 (Antiops). Type-locality: Uruguay, Montevideo. Distr. - same. Type o ZMB. N. COMB.

LINEARIS (Wulp), 1883:5 (Mesograpta). Type-locality: "Mexico". Distr. same. Type ??

LUNUS (Hull), 1943i:21, fig. 80 (abdomen), (Mesogramma). Type-locality: Puerto Rico, Mayaguez. Distr. - Puerto Rico, Guatemala. Type o USNM*. N. COMB.

LUTZI (Curran), 1930b:7 (Mesogramma). Type-locality: Panama, Canal 
Zone, Grijoles, Ft. Davis. Distr. - same. Type $+\mathrm{AMNH}^{*}$. Ref. Hull, 1943i:37, fig. 56 (abdomen). N. COMB.

var. FASciatus (Hull), 1948b:8 (Mesogramma). Type-locality: Venezuela, San Esteban. Distr. - same. Type o USNM*. N. COMB.

LYRATUS (Hull), 1940b:432 (Mesogramma). Type-locality: Panama, Canal Zone, Barro Colorado Island. Distr. - same. Type on Hull. Ref. Hull, 1943i:33, fig. 26 (abdomen). N. COMB.

MACQUARTI (Goot), 1964:214 (Mesograpta, nom. nov. for amoenus Macquart). Distr. - Venezuela, Peru, Paraguay, Bolivia. N. COMB.

amoenus Macquart, 1846:266 (138), pl. 13, fig. 1 (abdomen) (Syrphus; preocc. Loew, 1840). Type-locality: "Venezuela". Type \& OXF*.

Maculatus (Bigot), 1884b:111 (Mesograpta?). Type-locality: "Cuba, Mexico, Brazil". Distr. - Greater Antilles; Mexico south to Brazil and Argentina. Types $q$ OXF*. Ref. - Hull, 1943i:31, fig. 16 (abdomen).

MARGinatus (Say), 1823:92 (Scaeva). Type-locality: "U.S.A." Distr. Canada, U.S.A.; Grand Bahama Island, Mexico south to Peru. Type?? Ref. - Hull, 1943i:33, fig. 28 (abdomen).

circumcinctus Enderlein, 1938:232 (Mesogramma). Type-locality: Peru, Lima. Type ơ ZMB. N. SYN.

MELANOGASTER (Thomson), 1869:495 (Syrphus). Type-locality: Brazil, Rio de Janeiro. Distr. - same. Type o NRS*. N. COMB.

MINUTUS (Wiedemann), 1830:146 (Syrphus). Type-locality: "Brazil". Distr. - Cuba, Brazil. Type o SMF*.

MITIS (Curran), 1930b:13 (Mesogramma). Type-locality: Jamaica, Montego Bay. Distr. - same. Type o AMNH. Ref. - Hull, 1943i:47, fig. 75 (abdomen). N. COMB.

Mulio (Hull), 1941h:311 (Mesogramma). Type-locality: Brazil, São Paulo, Juquiá. Distr. - Colombia, Ecuador, Peru, Brazil, Argentina. Type $o^{*}$ Hull. Ref. - Hull, 1943i:29, fig. 2 (abdomen). N. COMB. MULTIPUNCTATUS (Wulp), 1883:6, pl. I, fig. 7 (abdomen) (Mesograpta). Type-locality: "Guadeloupe". Distr. - Guadeloupe, St. Vincent, Grenada. Type o ? Ref. - Hull, 1943i:38; fig. 66 (abdomen).

ferroxida Hull, 1942f:21 (Mesogramma). Type-locality: St. Vincent, British West Indies. Type o MCZ*.

MUSicus (Fabricius), 1805:253 (Scaeva). Type-locality: "South America". Distr. - Cuba, Jamaica, Puerto Rico, Virgin Islands, Trinidad; Mexico south to Brazil and Argentina. Types \& MC*. Ref. Hull, 1943i:31, fig. 21 (abdomen).

Mutuus (Say), 1829:164 (Syrphus). Type-locality: "Mexico". Distr. Mexico (Durango, Michoacán, Mexico City, Morelos, Vera Cruz, Chiapas), Guatemala. Type ?? Ref. - Hull, 1943i:43, fig. 60 (abdomen).

NASICUS (Hull), 1941i:432 (Mesogramma). Type-locality: Mexico, Oaxaca,

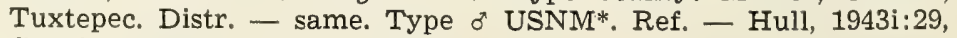
fig. 6 (abdomen). N. COMB.

NASUTUS Sack, 1941:102 (1951:98), fig. 4 (head), 5 (abdomen). Type-locality: Peru, Paso de Lares. Distr. - Ecuador, Peru, Bolivia. Types o MD? Ref. - Hull, 1943i:40, fig. 81 (abdomen).

sylphus Hull, 1943f:35 (Mesogramma). Type-locality: Ecuador, Baños. Type of AMNH*. N. SYN.

NIGRIPUNCTUS (Enderlein), 1938:231 (Mesogramma). Type-locality: Colombia, Cordillera. Distr: - same. Type $\sigma^{2}$ ZMB. N. COMB. 
NITIDIVENTRIS (Jurran), 1930b:9 (Mesogramma). Type-locality: Brazil, [?Espirito Santo], Vitória. Distr. - Colombia, Venezuela, Ecuador, Brazil. Type ơ AMNH*. N. COMB.

NITIDUS (Schiner), 1868:349 (Mesograma). Type-locality: "South America". Distr. - Panama. Types o* VMNH*.

NORMA (Curran), 1930b:10 (Mesogramma). Type-locality, Panama, Canal Zone, Corozal. Distr. - same. Type o AMNH*. Ref. - Hull, 1943i:29, fig. 7 (abdomen). N. COMB.

NYMPHALIUS (Hull), 1942a:106 (Mesogramma). Type-locality: Ecuador, Oriente, Río Margajitas, $1250 \mathrm{~m}$. Distr. - Ecuador, Surinam. Type o AMNH*. Ref. - Hull, 1943i:37, fig. 51 (abdomen). N. COMB.

OCHRACEUS (Hull), 1942f:19 (Mesogramma). Type-locality: Colombia, Bogotá. Distr. - same. Type o MCZ*. N. COMB.

octogutratus (Jaennicke), 1867:398 (Syrphus). Type-locality: "Chile". Distr. - Peru, Chile (Coquimbo-Chiloé), Argentina. Type ơ SMF*. Ref. - Shannon \& Aubertin, 1933:138, fig. 26b (abdomen); Hull, 1943i:37, fig. 57 (abdomen). N. COMB.

interruptus Philippi, 1865:747 (Syrphus; preocc. Gmelin, 1792). Typelocality: Chile, Valdivia, Illapel. Types $\delta^{\pi}$ \& MNHS.

philippii Shannon, 1927a:24 (Mesogramma; nom. nov. for interruptus Philippi).

OPHIOLINEA (Hull), 1943j:68, fig. 59 (abdomen) (Baccha). Type-locality: Colombia, Villavicencio. Distr. - same. Type o Hull*. Ref. Hull, 1949e: 202, fig. 59 (abdomen). N. COMB.

ORNATUS Sack, 1941:103 (1951:99), fig. 6 (abdomen). Type-locality: Peru; Bolivia; Brazil, Espirito Santo. Distr. - same. Types of $q$ MD ? and UNMB.

ORNITHOGLYPHUS (Hull), 1942f:17 (Mesogramma). Type-locality: Dominican Republic, Mount Diego de Ocampo, 3,000-4,000 ft. Distr. Cuba, Dominican Republic. Type o MCZ*. Ref. - Hull, 1943i:39, fig. 69 (abdomen). N. COMB.

ovatus (Hull), 1942f:19 (Mesogramma). Type-locality: Panama, Yape, Tuirar. Distr. - same. Type o MCZ*. Ref. - Hull 1943i:29, fig. 5 (abdomen), N. COMB.

PANAMENSIS (Curran), 1930b:12 (Mesogramma). Type-locality: Panama, Panama City. Distr. - same. Type o* AMNH*. N. COMB.

PARAGRAMmUS (Schiner), 1868:349 (Mesogramma). Type-locality: "South America". Distr. — same. Types o \% VMNH*.

PICTUS (Macquart), 1842:159 (99) pl. 16 fig. 11 (habitus) (Syrphus). Type-locality: "Guyane". Distr. - Greater Antilles, Lesser Antilles, Mexico south to Argentina. Type o MNHN*. Ref. - Hull, 1943i :30, fig. 13 (abdomen).

poecilogastrus Loew, 1865:158 (1872b:33) (Mesogramma). Type-locality: "Cuba". Type $+\mathrm{MCZ}$ *.

cuprinus Bigot, 1884b:114 (Mesograpta?). Type-locality: "Cuba". Type $\sigma^{*}$ OXF*. N. SYN.

var. MELleogutTatus (Hull), 1941f:45 (Mesogramma). Type-locality: Panama, Canal Zone, Barro Colorado Island. Distr. - same. Type o" Hull. Ref. - Hull, 1943i:31, fig. 15 (abdomen). N. COMB.

Politus (Say), 1823:88 (Scaeva). Type-locality: "United States". Distr. Canada, U.S.A.; Mexico south to Peru and Brazil. Type ? (lost). Ref. - Hull, 1943i:29, fig. 3 (abdomen). 
anchoratus Macquart, 1842:97 (132), pl. 16:8 (abdomen) (Syrphus). Type-locality: "Brazil". Types o $\sigma^{*}$ MNHN*. N. SYN.

POLYGRAPHICUS (Hull), 1940a:249 (Mesogramma). Type-locality: Brazil, São Paulo, Batéia. Distr. - Peru and Brazil. Type i Hull, 1943i:31, fig. 22 (abdomen). N. COMB.

PORTICola (Thomson), 1869:495 (Syrphus). Type-locality: Peru, Callao; Ecuador, Puna. Distr. - same. Type of NRS*. N. COMB.

PORTIUS (Walker), 1852:239 (Syrphus). Type-locality: "Brazil". Distr. Brazil (São Paulo). Type o" BMNH*. Ref. - Hull, 1943i:31, fig. 17 (abdomen), N. COMB.

PRoductus (Curran), 1930b:5 (Mesogramma). Type-locality: Ecuador. Distr. - Ecuador south to Argentina. Type o AMNH*. Ref. Hull, 1943i:32, fig. 30 (abdomen). N. COMB.

PUEllus (Hull), 1942f:22 (Mesogramma). Type-locality: Cuba, Santa Clara Province, Soledad. Distr. - same. Type o MCZ*. Ref. - Hull, 1943i:39, fig. 64 (abdomen). N. COMB.

PULCHELluS (Macquart), 1846:266 (138), pl. 11, fig. 12 (abdomen) (Syrphus). Type-locality: Guadeloupe, San Domingo. Distr. - Guadeloupe, Cuba. Types o OXF*. Ref: - Hull, 1943i:34, fig. 46 (abdomen).

PUNCTATUS Sack, 1921:128, fig. 1a (abdomen). Type-locality: Paraguay, St. Trinidad. Distr. - same. Types ot $f$ MD?

PuRus (Curran), 1930b:10 (Mesogramma). Type-locality: Panama, Corozal. Distr. - same. Type o AMNH. Ref. - Hull, 1943i:39, fig. 63 (abdomen). N. COMB.

QUADRILINEATUS (Enderlein), 1938:233 (Hybobathus). Type-locality: Colombia, "Cordillera, tierra templada". Distr. - same. Types ๆ ZMB. N. COMB.

QUINQUECINCTUS (Bigot), 1888a:254 (Mesograpta). Type-locality: Mexico. Distr. - same. Type $+\mathrm{OXF}^{*}$.

QUINQUEMACUlatus (Bigot), 1888a:254 (Mesograpta). Type-locality: "Mexico". Distr. - same. Types of o OXF*.

RHEA (Hull), 1949i:228 (Mesogramma). Type-locality: Brazil, Santa Catarina, Nova Teutônia. Distr. - same. Types $\sigma^{*}$ AMNH*, MZUSP. N. COMB.

RHIODOPE (Hull), 1951a:13 (Mesogramma). Type-locality: Jamaica, Dunrobin District, Mandeville, Manchester. Distr. - same. Type $\%$ AMNH*. N. COMB.

Rombicus (Giglio-Tos), 1892d:3 (Mesogramma). Type-locality: Mexico, Orizaba. Distr. - same. Types of of UTOR*. Ref. - Giglio-Tos, 1893:pl. 2, fig. 15 (abdomen); Hull, 1943i:40, fig. 78 (abdomen).

rhombicus, various authors, emend.

RUfiCAUdATU (Bigot), 1884a:541 (Paragus). Type-locality: "Brazil". Distr. - same. Type o $\mathrm{BM}(\mathrm{NH}) *$. N. COMB.

RUfocinctus (Curran), 1927b:7 (Mesogramma). Type-locality: Puerto Rico, Mayaguez. Distr. - same. Type o $\mathrm{AMNH}^{*}$. Ref. - Hull, 1943i:41, fig. 83 (abdomen). N. COMB.

SAPHIRIDICEPS (Bigot), 1884b:105 (Mesograpta?). Type-locality: "Mexico". Distr. - Mexico, Colombia, Ecuador, Peru. Types + OXF*.

SElva (Hull), 1943i:13 (Mesogramma). Type-locality: ? Distr. - ? Type ?? N. COMB. 
SERPENTINUS (Hull), 1950c:228 (Calostigma). Type-locality: Peru, Chanchamayo. Distr. - same. Type of Hull*. N. COMB.

SINUATINERViS (Macquart), 1850:455 (151), tab. 14, fig. 10 (wing) (Syrphus). Type-locality: Brazil, "Minas Gerais". Distr. - same. Type o MNHN. N. COMB.

SLOSSONAE (Curran), 1930b:8 (Mesogramma). Type-locality: U.S.A., Florida, Biscayne Bay. Distr. - U.S.A. (Florida), Ecuador. Type o" AMNH*. N. COMB.

STEATOGASTER (Iull), 1941c:62 (Mesogramma). Type-locality: Brazil, Amazonas, Rio Branco. Distr. - Ecuador, Brazil. Type o Hull. Ref. Hull, 1943i:29, fig. 1 (abdomen). N. COMB.

STEATORNIS (Hull), 1943i:20 (Mesogramma). Type-locality: Ecuador, Santiago-Zamora, Sucua, Río Blanco and Río Upano, $950 \mathrm{~m}$. Distr. same. Type of AMNH*. N. COMB.

SUBANNULATUS (Loew), 1865:157 (1872b:31) (Mesograpta). Type-locality: "Cuba". Distr. - Cuba, Dominican Republic, Jamaica, Puerto Rico, Costa Rica south to Brazil. Types of o MCZ*. Ref. - Hull, 1943i:33, figs. 34,35 (abdomen).

Sylvaticus (Hull), 1943f:34 (Mesogramma). Type-locality: Ecuador, Baños. Distr. - same. Type o AMNH*. Ref. - Hull, 1943i:41, fig. 81 (abdomen). N. COMB.

TAENIUS (Curran), 1930b:7, fig. 3 (ventral view of male genitalia) (Mesogramma). Type-locality: Panama, Canal Zone, Corozal. Distr. Panama, Surinam, Argentina. Type $0^{x} \mathrm{AMNH}^{*}$. Ref. - Hull, 1943i:29, fig. 4 (abdomen). N. COMB.

TIBICEN (Wiedemann), 1830:127 (Syrphus). Type-locality: "Brazil". Distr. - Panama (Canal Zone) south to Brazil and Argentina. Types $q$ ZMB-VM(NH)*. Ref. - Hull, 1943i:35, fig. 43 (abdomen).

TRIANGUlatus (Hull), 1942a:104 (Mesograma). Type-locality: Paraguay, Villarica. Distr. - same. Type o AMNH*. Ref. - Hull, 1943i:37, fig. 55 (abdomen). N. COMB.

TRIDENTATUS (Rondani), 1868:24 (Syrphus). Type-locality: "Patagonia". Distr. - Mexico, "Patagonia". Type o" ? Ref. - Giglio-Tos, 1893: pl. 2, fig. 17 (abdomen). N. COMB.

pallipes Bigot, 1884b:106 (Mesograpta?). Type-locality: "Mexico". Type $+\mathrm{OXF}^{*}$.

"RILINEATUS (Enderlein), 1938:232 (Mesogramma). Type-locality: "St. Jean". Distr. - same. Type o ZMB. N. COMB.

Trilobatus (Bigot), 1884b:109 (Mesograpta?). Type-locality: "Mexico". Distr. - same. Types o OXF*.

TRIRAdiatus (Hull), 1942d:48 (Mesogramma). Type-locality: Panama, Canal Zone, Barro Colorado Island. Distr. - same. Type $\sigma^{7}$ Hull. Ref. - Hull, 1943i:33, fig. 27 (abdomen). N. COMB.

TUBUlarius (Hull), 1942a:103 (Mesogramma). Type-locality: Ecuador, Baños. Distr. - same. Type o" AMNH*. Ref. - Hull, 1943i:35, fig. 48 (abdomen). N. COMB.

ultamus (Hull), 1951a:7 (Mesogramma). Type-locality: Ecuador, Pichincha, Uyumbicho. Distr. - same. Type \& AMNH*. N. COMB.

UNA (Hull), 1943i:23, fig. 84 (abdomen) (Mesogramma). Type-locality: "Haiti". Distr. - same. Type o MCZ*. N. COMB.

UNDECIMPUNCTATUS (Enderlein), 1938:228 (Mitrosphen). Type-locality: Uruguay, Montevideo; Paraguay, San Bernardino. Distr. - same. Types of $\&$ ZMB. N. COMB. 
URANIUS (Hull), 1951a:1 (Mesogramma). Type-locality: Peru, middle Río Ucayali. Distr. - same. Type o AMNH*. N. COMB.

VALDESI (Fluke), 1950b:488, fig. 12 (abdomen), fig. 13 (metathoracic leg) (Mesogramma). Type-locality: Cuba, Pico Joaquin to Turquino. Distr. - same. Type o AMNH*. N. COMB.

VANESSA (Hull), 1951a:15 (Mesogramma). Type-locality: Brazil, Santa Catarina, Nova Teutônia. Distr. - same. Type, q AMNH*. N. COMB.

VATIUS (Walker), 1852:240 (Syrphus). Type-locality: Brazil. Distr. - same. Type o $\mathrm{BM}(\mathrm{NH}) *$. Ref. - Hull, 1943i:29, fig. 8 (abdomen). N. COMB.

VERTICALIS (Curran), 1927b:6 (Mesogramma). Type-locality: Puerto Rico, Gayay. Distr. - same. Type o AMNH*. Ref. - Hull, 1943i:41, fig, 76 (abdomen). N. COMB.

verticallis, Hull 1943i, misspelled.

VEVE (Hull), 1942f:23 (Mesogramma). Type-locality: Haiti, La Visite and Vic., La Salle Range 5,000-6,000 ft. Distr. - same. Type o* MCZ*. N. COMB.

viciNuS (Macquart), 1846:264 (136) (Syrphus). Type-locality: "Brazil". Distr. - same. Type o $\mathrm{OXF}$. N. COMB.

vIERECKI (Curran), 1930b:13 Mesogramma). Type-locality: Colombia, Sero Quenado. Distr. - same. Type \& AMNH*. Ref. - Hull, 1943i:37, fig. 58 (abdomen). N. COMB.

violaCeus (Curran), 1926c:103 (Mesogramma). Type-locality: Jamaica, Chinchona. Distr. - Jamaica, Puerto Rico, Dominican Republic. Type $o^{*}$ BM(NH). Ref. - Curran, 1928:39, fig. 5 (head): 40, fig. 6 (abdomen). N. COMB.

virgulatus (Macquart), 1850:456 (152), pl. 14, fig. 11 (abdomen) (Syrphus). Type-locality: "Patrie inconnue, probablement le Brésil". Distr. - same. Type o $\mathrm{OXF}^{*}$. N. COMB.

vitrecens (Hull), 1930:142 (Mesogramma). Type-locality: Colombia, Aracataca, Magdalena. Distr. - same. Type o ANSP. N. COMB.

vitreus (Hull), 1941f:45 (Mesogramma). Type-locality: Brazil, São Paulo, Juquiá. Distr. - same. Type o Hull. N. COMB.

vulturus (Hull), 1942f:20 (Mesogramma). Type-locality: Honduras, Lancetilla, near Tela. Distr. - same. Type o MCZ*. Ref. - Hull, 1943i:39, fig. 70 (abdomen). N. COMB.

WATSONI (Curran), 1930b:5, fig. 1 (ventral view of male genitalia) (Mesogramma). Type-locality: Haiti, Aux Cayes. Distr. - Haiti, Cuba, Jamaica, Venezuela, Colombia, Ecuador, Peru, Brazil. Type ot AMNH*. Ref. - Hull, 1943i:33, fig. 32 (abdomen). N. COMB.

WILlistoniI (Lynch Arribálzaga), 1892a:54 (1892c:64) (Mesogramma). Type-locality: Argentina, Misiones. Distr. - same. Type ? MACN.

\section{Subfamily MICRODONTINAE \\ Ref. - Thompson, 1969 (key to general). \\ Genus PARAgODON Thompson}

PARAGODON Thompson, 1969:74. Type-species, paragoides Thompson (orig. des.). Ref. - Thompson, 1969 (key). 
MiNutulus (Doesburg), 1966:89, figs. 63 \& 64 (habitus) (Ceratophya). Type-locality: Surinam, Zanderij-savannah, Distr. - same. Type o Doesburg.

PARAGOIDES Thompson, 1969:81, figs. 1, 1a (head); 7, 7a (male genitalia); 9 (abdomen); 12 (wing). Type-locality: Mexico, Sinaloa, Mazatlán, at sea level. Distr. - same. Type o $\mathrm{CNC}^{*}$.

\section{Genus Paramicrodon Meijere}

PARAMICroDon Meijere, 1913:360. Type-species, lorentzi Meijere (mon.).

Spheginobaccha, subg. Nannomyrmecomyia Hull, 1945b:75. Type-species, Paramicrodon delicatulus Hull (orig. des.). N. SYN.

DELICATUlus Hull, 1937b:24. Type-locality: Cuba, Soledad (Cienfuegos). Distr. - same. Type ơ MCZ*. Ref. - Thompson, 1969:77, fig. 2 (head).

FlUKeI (Curran), 1936b:2 (Microdon). Type-locality: Brazil, Rio de Janeiro, Petrópolis. Distr. - Peru, Brazil (Rio de Janeiro, Santa Catarina). Type o AMNH*. N. COMB.

\section{Genus Argentinomyia Lynch Arribálzaga}

ARgentinomyia Lynch Arribálzaga, 1891:199 (1892c:30). Type-species, testaceipes Lynch Arribálzaga (mon.).

Aristosyrphus Curran, 1941:252. Type-species, primus Curran (orig. des.). N. SYN.

Ceratophya, subg. Protoceratophya Hull, 1949d:314. Type-species, carpenteri Hull (orig. des.). N. SYN.

Paraceratophya, Fluke, 1957:38, misspelling of Protoceratophya.

\section{Subgenus ARGENTINOMYia Lynch Arribálzaga}

BORACEIENSIS (Papavero), 1962:319 (Ceratophya). Type-locality: Brazil, São Paulo, Salesópolis, Estação Biológica de Boracéia. Distr. same. Type of MZUSP*. N. COMB.

CARPENTERI (Hull), 1945b:76 (Ceratophya). Type-locality: Panama, Coclé Province, El Valle. Distr. - same. Type of $\mathrm{MCZ}^{*}$. N. COMB.

EloNgata (Hull), 1943e:706 (Microdon). Type-locality: Brazil, Pará, Santarém. Distr. - same. Type ơ BM(NH). N. COMB.

GRANDIS Lynch Arribálzaga, 1892b:255 (1892c:156). Type-locality: Argentina, Misiones, Fracrao. Distr. - same. Type ? MACN.

MACroptera (Curran), 1941:254 (Ceratophya). Type-locality: Brazil, Santa Catarina, Nova Teutônia. Type \& AMNH*. N. COMB.

PRIMA (Curran), 1941:252 (Aristosyrphus). Type-locality: Brazil, Santa

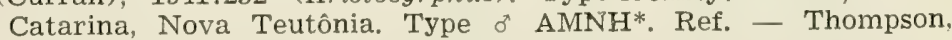
1969:82, fig. 11 (wing). N. COMB.

TESTACEIPES Lynch Arribálzaga, 1891:199 (1892c:41), fig. 4 (habitus). Type-locality: Argentina, Prov. Buenos Aires, Chacabuco. Distr. same. Type? MACN.

\section{Subgenus EURYPTERosyrenus Barretto \& Lane}

EURYPTEROSYRPHUS Barretto \& Lane, 1947:141 (as genus). Type-species, melanopterus Barretto \& Lane (orig. des.). 
MELANOPTERA (Barretto \& Lane), 1947:142, fig. 1 (wing) (Eurypterosyrphus). Type-locality: Brazil, São Paulo, São Paulo, Via Anhanguera. Distr. - same. Type \& MZUSP*. N. COMB.

\section{Genus MrXogaster Macquart}

MIXOGASTER Macquart, 1842:74 (14). Type-species, conopsoides Macquart (orig. des.).

Myxogaster, Kertész, 1910:351, misspelling.

Ref. - Hull, 1954 (rev.), Carrera \& Lane, 1958 (key).

ANTHERMuS (Walker), 1849:547 (Ascia). Type-locality: Brazil, Pará. Distr. - Brazil. Types \& BM(NH). Ref. - Hull, 1954:21 (notes).

CrCATrix Hull, 1954:9 figs. 14 (head), 26 (abdomen). Type-locality: Brazil, Mato Grosso, Corumbá. Distr. - -same. Type ơ CU*.

CONopsoides Macquart, 1842:74 (14), tab. 3, fig. 1 (habitus), 1a (head). Type-locality: Brazil, Rio de Janeiro, Distr. - Colombia, Brazil. Type o MNHN*. Ref. - Hull, 1954:19 (translation of original description).

conopseus, Williston, 1886:309, 310; misspelling.

conopoides, Kertész, 1910:351, emendation.

Cubensis Curran, 1932a:1. Type-locality: Cuba, Sierra Maestra. Distr. Cuba. Type o" AMNH*. Ref. - Hull, 1954:14 (redescription), fig. 8 (antenna); 27 (abdomen); Thompson, 1969:76, fig. 4 (metasterna).

CURRANI Hull, 1954:5, figs. 9 (antenna), 16 (head), 31 (abdomen). Typelocality: Brazil, Santa Catarina, Nova Teutônia. Distr. - same. Type o" AMNH*.

dimidiata Giglio-Tos, 1892c:1. Type-locality: Mexico, Tuxpango. Distr. same. Type \& UTOR. Ref. - Hull, 1954:19 (transl. of orig. description).

dimitiata, Fluke, 1957:37, misspelling.

FLUKEI Hull, 1954:15, figs. 2 \& 17 (head), 22 (wing), 28 (abdomen). Type-locality: Brazil, São Paulo. Distr. - same. Type $\sigma^{*}$ AMNH.

LANEI Carrera \& Lenko, 1958:473, figs. 4 (egg), 5-7 (larva), 8-9 (puparium), 10-11 (head), 12 (antenna), 13 (wing). Type-locality: Brazil, São Paulo, Campos do Jordão. Distr. - Brazil (São Paulo). Type $\sigma^{*}$ MZUSP*.

LOPESI Carrera \& Lenko, 1958:477, figs. 14-15 (head), 17 (wing). Typelocality: Brazil, Pará, Belém, Aurá. Distr. - same. Type ơ IOC.

MEXICANA Macquart, 1846:251 (123), pl. 10, fig. 15 (head), 15a (wing). Type-locality: Mexico. Distr. - same. Type \& MRHNB. Ref. Hull, 1954:20 (translation of orig. description).

ORPHeUs Hull, 1944c:36. Type-locality: Guyana, Bartica. Distr. - same. Type \& MCZ*. Ref. - Hull, 1954:21 (redescription); fig. 19 (wing).

PITHECOFASCIA Hull, 1944k:512. Type-locality: Colombia, Restrepo, $500 \mathrm{~m}$. Distr. - same. Type o Hull. Ref. - Hull, 1954:23 (redescription), figs. 3 \& 15 (head), 32 (abdomen).

POLISTES Hull, 1954:4, figs. 5 \& 12 (head), 8 (antenna), 21 (wing). Typelocality: Brazil, Guanabara, Rio de Janeiro. Distr. - same. Type o $\mathrm{AMNH}$ * 
RARIOR Shannon, 1925a:111. Type-locality: Panama, Taboga Island. Distr. - Panama. Type o" USNM*. Ref. - Hull, 1954:11 (redescription), figs. 20 (wing), 25 (abdomen).

var. RARISSIMA Shannon, 1925a:111. Type-locality: Guatemala, Cacao Trece Aguas, Alta Vera Paz. Type ơ USNM*.

SARTOCRYPTA Hull, 1954:8, figs. 1 \& 11 (head), 6 (antenna), 29 (abdomen). Type-locality: Brazil, Santa Catarina, Nova Teutônia. Distr. Brazil. Type क AMNH*.

STRICTOF Hull, 1941a:1. Type-locality: Bolivia, Santa Cruz, Piedra Blanca (as "Brazil", Piedra"). Distr. - same. Type o" AMNH*. Ref. Hull, 1954:17 (redescription), fig. 18 (head).

THECLA Hull, 1954:6, figs. 13 (head), 23 (wing), 30 (abdomen). Typelocality: Brazil, Guanabara, Rio de Janeiro. Distr. - Ecuador, Peru, Brazil. Type of AMNH*.

\section{Genus Microdon Meigen}

MICRODON Meigen, 1803:275. Type-species, Musca mutabilis Linnaeus (mon.).

Chymophila Macquart, 1834:485. Type-species, splendens Macquart (mon.) = fulgens. Wiedemann.

Chimophila, Osten-Sacken, 1875:46, 1878:119, misspelling.

Omegasyrphus Giglio-Tos, 1891:4. Type-species, Microdon coarctatus Loew (sub. mon., Giglio-Tos, 1892b:3).

Microdon, subg. Eumicrodon Curran, 1925a:50. Type-species, fulgens Wiedemann (orig. des.).

Pseudomicrodon Hull, 1937b:24. Type-species, Microdon beebei Curran (orig. des.). N. SYN.

Chrysidimyia Hull, 1937c:116 (as Chysidimyia). Type-species, chrysidimima Hull (orig. des.). [Even though Hull spelled his generic name as Chysidimy:a in the original description (3 times!) we consider this as a lapsus calami. Hull did refer to the "astonishing resemblance" of his group to chrysidid wasps and thereby indicated the intended derivation of his name. All subsequent spellings of this name (Hull, 1938:126, 1944e:241, 1949d:309) are as Chrysidimyia, which we here accept as a justified emendation].

Syrphipogon Hull, 1937c:120. Type-species, fucatissimus Hull (orig. des.). Ref. - Curran, 1941 (keys).

\section{Subgenus Ceratophya Wiedemann}

CERATOPHYA Wiedemann, 1824:14 (as genus). Type-species, notata Wiedemann (Coquillett, 1910a:520).

Cerathophya, Wiedemann, 1830:79, misspelling.

CARINIFACIES Curran, 1934b:376. Type-locality: Guyana, Kartabo. Distr. same. Type \& AMNH*.

LONGICORNIS (Wiedemann), 1824:14 (Ceratophya). Type-locality: "Brazil". Distr. - same. Type + VMNH. N. COMB.

Notatus (Wiedemann), 1824:14, fig. 9 (habitus, antenna, scutellum) (Ceratophya). Type-locality: "Brazil". Distr. - same. Type o VMNH*.

PANAMENSIS Curran, 1930f:6. Type-locality: Panama, Canal Zone, France Field. Distr. - same. Type o AMNH*. 


\section{Subgenus RHOPALOSYRPHUS Giglio-Tos}

RHOPALOSYrPhus Giglio-Tos, 1891:3 (as genus). Type-species, Holmbergia guentherii Lynch Arribálzaga (sub. mon., Giglio-Tos, 1892b:1).

Holmbergia Lynch Arribálzaga, 1891:195 (1892c:37). Type-species, guentherii Lynch Arribálzaga (mon.).

Ref. - Capelle, 1956 (key).

CAROLAE (Capelle), 1956:174, figs. 3 (head), 4 (wing), 5 (scutellum) (Rhopalosyrphus). Type-locality: U.S.A., Arizona, Huachuca Mountains, Sunnyside Canyon. Distr. - U.S.A. (Arizona), Mexico. Type $\subseteq \mathrm{UK}^{*}$. N. COMB.

GUENTHERII (Lynch Arribálzaga), 1891:197 (1892c:39), fig. 3 (habitus) (Holmbergia). Type-locality: Argentina, Buenos Aires. Distr. Argentina, Surinam, Mexica. Type ? MACN. Ref, - Hull, 1949d: 315 , figs. $14 \mathrm{f}$ (habitus), $14 \mathrm{~g}$ (hind leg), 14h (head), 14i (abdomen); Doesburg, 1966:88, fig. 61 [not 62] (habitus); Thompson, 1969:76, fig. 6 (metasterna). N. COMB.

\section{Subgenus CerIomicrodoN Hull}

CERIOMICRODON Hull, 1937b:25 (as genus). Type-species, petiolatus Hull (orig. des.).

Cerioimicrodon, Hull, 1937b25, incorrect original spelling.

PETIOLATus (Hull), 1937b:25 (Cerioimicrodon). Type-locality: Brazil, west border of Mato Grosso. Distr. - Peru, Brazil (Mato Grosso). Type o USNM*. Ref, - Hull, 1949d:313, fig. 13e (habitus). N. COMB.

\section{Subgenus RHOGA Walker}

RHOGA Walker, 1857:157. Type-locality, Iutescens Walker (mon.).

Papiliomyia Hull, 1937b:27. Type-species, sepulchrasilva Hull (orig. des.). LUtescens (Walker), 1857:157 (Rhoga). Tpe-locality: Brazil, Pará. Distr. - same. Type \& $\mathrm{BM}(\mathrm{NH})$. N. COMB.

Maculatus Shannon, 1927a:21. Type-locality: Bolivia, Prov. Sara, Santa Cruz de la Sierra. Distr. - same. Type o $\mathrm{BM}(\mathrm{NH})$.

Melleus Curran, 1940:5. Type-locality: Guyana, Kaieteur, Tukheit Trail. Distr. - Costa Rica, Panama, Guyana. Type ơ BM(NH).

SEPUlChrasilvus (Hull), 1937b:28 (Papiliomyia). Type-locality: Brazil, Rio Grande do Sul. Type o VMNH*. Ref. - Full, 1949d:315, fig. $14 \mathrm{~d}$ (habitus). N. COMB.

XANTHOPROSOPUS (Barretto \& Lane), 1947:145 (Rhoga). Type-locality: Brazil, Minas Gerais, Arceburgo, Faz. Fortaleza. Distr. - same. Type o MZUSP*. N. COMB.

\section{Subgenus Masarygus Brèthes}

MASARYGuS Brèthes, 1908:441 (as genus). Type-species, planifrons Brèthes (orig. des.).

Schizoceratomyia Carrera, Lopes \& Lane, 1947a:245. Type-species, barrettoi Carrera, Lopes \& Lane (orig. des.). 
Johnsoniodon Curran, 1947a:1. Type-species, malleri Curran (orig. des.). Ref. - Papavero, 1962 (key).

BARRETTOI (Carrera, Lopes \& Lane), 1947a:245; 1947b:473, figs. 1 (habitus); 2-3 (head); 4-5, 13 (antenna); 6 (wing); 7-11 (male genitalia) (Schizoceratomyia). Type-locality: Brazil, Goiás, Corumbá, Fazenda Monjolinho. Distr. - Surinam, Brazil (Goiás, Rio de Janeiro. Type o MZUSP*. N. COMB.

CARRERAI (Papavero), 1962:324, fig. 4 (antenna) (Masarygus). Type-locality: Brazil, São Paulo, Salesópolis, Estação Biológica de Boracéia.

Distr. - same. Type o MZUSP*. N. COMB.

Flavipes (Carrera, Lopes \& Lane), 1947a:247; 1947b:476, figs. 14 (head), 15-16 (antenna), 17-19 (male genitalia) (Schizoceratomyia). Typelocality: Brazil, São Paulo, Alto da Serra, Parque Cajuru. Distr. - same. Type ơ MZUSP*. Ref. - Doesburg, 1966:92, fig. 65 (habitus). N. COMB.

MALLERI (Curran), 1947a:1, fig. 1 (habitus) (Johnsoniodon). Type-locality: Brazil, Santa Catarina, Corupá (Hansa Humboldt). Distr. same. Type of AMNH*. N. COMB.

PLANIFRONS (Brèthes), 1908:442, figs. a-c (habitus), $d+e$ (head), $f+g$ (antenna) (Masarygus). Type-locality: Argentina, Buenos Aires, General Urquiza. Types o $\%$ MACN \& MCSNM*.

\section{Subgenus UBRISTES Walker}

UBRISTES Walker, 1852:217 (as genus). Type-species, flavitibia Walker (mon.).

Hypselosyrphus Hull, 1937b:21. Type-species, trigonus Hull (orig des.). Ubristes, subg. Stipomorpha Hull, 1945b:74. Type-species, Microdon fraudator Shannon (orig: des.).

Carreramyia Doesburg, 1966:93. Type-species, Microdon megacephalus Shannon (orig. des.). N. SYN.

ANAX Thompson (nom. nov, for analis Curran). Distr. - Brazil (Santa Catarina). NOM. NOV.

analis Curran, 1940:3 (preocc. Macquart, 1842). Type-locality: Brazil, Santa Catarina, Nova Teutônia. Type o AMNH*.

angulatus Hull, 1943e:715. Type-locality: Brazil, "Amazon, Ega" (= Tefé). Distr. - same. Type o $\mathrm{BM}(\mathrm{NH})$.

APICUlus Curran, 1930f:5. Type-locality: Panama, Canal Zone, Barro Colorado Island. Distr. - same. Type o AMNH*.

CHRYsopygus (Giglio-Tos), 1892c:1 (Ubristes). Type-locality: Mexico, Orizaba. Distr. - same. Type o UTOR. N. COMB.

CORBICUlipes (Papavero), 1962:320, figs. 2,3 (hind tibia + tarsus) (Hypselosyrphus). Type-locality: Brazil, Guanabara, Sumaré. Distr. same. Type o MZUSP*. N. COMB.

FlavitiBia (Walker) 1852:217, pl. 5:2 (habitus), 2a (head), 2b (tarsus) (Ubristes). Type-locality: "Brasil". Distr. - Guyana, Brazil. Type or BM(NH). Ref. - Hull, 1949d:313, figs. 13f (hind leg), $13 \mathrm{~h}$ (abdomen).

plaumanni Curran, 1940:3. Type-locality: Brazil, Santa Catarina, Nova Teutônia. Type \& AMNH*. N. SYN.

FraUdator Shannon, 1927a:20. Type-locality: "Amazon". Distr. - same. Type o BM(NH). Ref. - Hull, 1949d:313, fig. 13g (abdomen).

GoETtEI Shannon, 1927a:19. Type-locality: "Amazon". Distr. - same. Type $₹ \mathrm{BM}(\mathrm{NH})$. 
GUIANICUS Curran, 1925c:340. Type-locality: Guyana, Bartica. Distr. Panama, Ecuador, Surinam, Guyana. Type o MCZ*. Ref. Thompson, 1969:76, fig. 3 (head).

inARMATUS Curran, 1925d:5. Type-locality: Guyana, Bartica. Distr. Guyana. Type $0^{*} \mathrm{MCZ}$ *

LACTEIPENNIS Shannon, 1927a:18. Type-locality: "Amazon". Distr. - same. Type o $\mathrm{BM}(\mathrm{NH})$.

LANEI Curran, 1936b:5. Type-locality: Brazil, São Paulo, Juquiá. Distr. Trinidad, Brazil (São Paulo). Type o $\mathrm{AMNH}^{*}$.

MACKIEI Curran, 1940:5. Type-locality: Guyana, Rockstone. Distr. - same. Type of AMNH*.

MEgaCePHalus Shannon, 1925b:213. Type-locality: Panama, "Old Panama". Distr. - same. Type o USNM*. Ref. - Hull, 1949d:313, figs. 13a (habitus), 13b (hind leg); 13c (head).

MICROMIDAS Shannon, 1925a:112. Type-locality: Panama, Taboga Island. Distr. - same. Type o USNM*.

Mixtus Curran, 1940:6. Type-locality: Guyana, Cuyuni River, Kamaria Landing. Distr. - same. Type of $\mathrm{BM}(\mathrm{NH})$.

PRoCedens Curran, 1941:251. Type-locality: Brazil, Santa Catarina, Nova Teutônia. Distr. - same. Type ơ AMNH*.

PRoCTERI Curran, 1941:251. Type-locality: Brazil, Santa Catarina, Nova Teutônia. Distr. - same. Type ơ AMNH*.

PUERILIS (Doesburg), 1966:86 (Ubristes). Type-locality: Surinam, Zanderij. Distr. - same. Type $q$ Doesburg. N. COMB.

scolopus Shannon, 1927a:20. Type-locality: "Amazon". Distr. - same. Type o $\mathrm{BM}(\mathrm{NH})$.

SCUTELlARIS Shannon, 1927a:20. Type-locality: "Amazon". Distr. - same. Type o $\mathrm{BM}(\mathrm{NH})$. Ref. - Hull, 1949d:313, figs. 13d (head), 13i (scutellum), 13j (head), 13k (hind leg).

SIMrlumus Hull, 1950:611. Type-locality: Guyana, Essequibo River, Moraballi Creek. Distr. - same. Type o BM (NH).

TENuICaudus Curran, 1925c:339. Type-locality: Peru, El Campamiento. Distr. - Peru, Brazil. Type o+ CU*. Ref. - Curran, 1934b:526, figs. 26 (abdomen), 27 (head).

Tritangularis Curran, 1940:6. Type-locality: Brazil, Mato Grosso, Dourados. Distr. - Brazil (São Paulo, Mato Grosso). Type ơ AMNH*.

litoralis Papavero, 1964:21, figs. 1,2 (abdomen) (Ubristes). Typelocality: Brazil, São Paulo, Caraguatatuba, $40 \mathrm{~m}$. Type $\sigma^{\pi}$ MZUSP*. N. SYN.

TRIGONIFORMIS Shannon, 1927a:19. Type-locality: Brazil, ?Vila Nova. Distr. - same. Type o $\mathrm{BM}(\mathrm{NH})$.

TRIGONUS (Hull), 1937b:21, fig. 9 (head), 9a (scutellum) (Hypselosyrphus). Type-locality: Panama, Canal Zone, Barro Colorado Island. Distr. - same. Type $\sigma^{*} \mathrm{MCZ}^{*}$. N. COMB.

Uloponus (Hull), 1944j:34 (Ubristes). Type-locality: Peru, Putumayo Dist., Lachoruria. Distr. - same. Type o CU*. N. COMB.

Wheeleri Mann, 1928:168. Tpe-locality: Panama, Canal Zone, Red Tank. Distr. - same. Type ơ USNM*.

\section{Subgenus Microdon Meigen}

ABNORMIS Curran, 1925c:345. Type-locality: Venezuela, Caura Valley. Distr. - same. Type \& MCZ*.

AEOLIDIFORMIS Wheeler, 1924:239, fig. 1 (larva, puparium). Type-locality: Panama, Canal Zone, Frijoles. Distr. - same. Type (larva) USNM. 
ANGUSTIVENTRIS (Macquart), 1855:105 (85) (Aphritis). Type-locality: "Amérique méridionale". Distr. - Guyana. Type o OXF*.

ANGustus (Macquart), 1846:250 (122) (Aphritis). Type-locality: "Cayenne". Distr. - Colombia, Guyana. Type o. MNHN?

AQUILINUS Giglio-Tos, 1892c:2. Type-locality: Mexico, Tuxpango. Distr. same. Type o UTOR.

argentinae Hull, 1937b:18. Type-locality: Argentina, Córdoba. Distr. same. Type ot MCZ*.

Aureoscutus Hull, 1943e:709. Type-locality: "Amazon". Distr. - same. Type ơ $\mathrm{BM}(\mathrm{NH})$.

AUreuS Hull, 1944c:35. Type-locality: Ecuador, Oriente, Río Naxo, Jatun Yacu, Watershed, $700 \mathrm{~m}$. Distr. - same. Type of $\mathrm{MCZ} *$.

AURICINCTUS (Sack), 1931:148, figs. 23a (head), 24 (habitus) (Rhopalosyrphus). Type-locality: Paraguay, St. Trinidad. Distr. - same. Type of DEI. N. COMB.

AURIFACIUS Hull, 1937a:169. Type-locality: Brazil, São Paulo, Itaquaquecetuba. Distr. - same. Type o USNM*.

AURIFAScra Hull, 1944e:245. Type-locality: Brazil, São Paulo, Alto da Serra (= Paranapiacaba). Distr. - same. Type o $\mathrm{BM}(\mathrm{NH})$.

AURIFEX Wiedemann, 1830:85. Type-locality: "Brazil". Distr. - Mexico (Guerrero), Brazil. Type o VMNH*. Ref. - Williston, 1891:pl. 1, fig. 2 (habitus), 2a (head).

trochilus Walker, 1852:216. Type-locality: "Mexico". Type o BM(NH). AURIGASTER Hull, 1941g:160. Type-locality: Bolivia, Prov. Sara. Distr. same. Type o MCZ*.

aurulentus (Fabricius), 1805:185 (Mulio). Type-locality: U.S.A., "Carolina". Distr. - U.S.A. (New York to North Carolina); ?Mexico [Giglio-Tos, 1892e:35] (probably not neotropical). Type + (lost?). Ref. - Curran, 1925a:80 (redescription), 196, fig. 41 (antenna). RaLIOPTERUS Loew, 1872a:86 (1872b:262). Type-locality: U.S.A., Texas. Distr. - U.S.A. (California to Verginia, south to Florida); Mexico. Types or $q$ MCZ (lost)*. Ref. - Curran, 1925a:87 (redescription), pl. 5:47 (scutellum + antenna), 9:47 (male genitalia).

var. BRUNNIPENNIS Hull, 1944i:400. Type-locality: Guatemala, St. Thomas. Type $0^{*}$ VMNH*.

BANKSI Hull, 1942a:91. Type-locality: Cuba, coast below Pico Turquino. Distr. - same. Type of MCZ*.

Barbiellinin Curran, 1936b:6. Type-locality: Brazil, São Paulo. Distr. same. Type o $\mathrm{AMNH}^{*}$.

Barbouri Hull, 1942a:89. Type-locality: Brazil, Paraná, Paranaguá. Distr. - same. Type + $\mathrm{MCZ}$ *

BASSLERI Curran, 1940:10. Type-locality: Peru, Río Ucayali. Distr. - same. Type \& AMNH*.

BATESI Shannon, 1927a:22. Type-locality: Brazil, Amazon. Distr. - same. Type o $\mathrm{BM}(\mathrm{NH})$.

BEeBei Curran, 1936b:4. Type-locality: Guyana, Kartabo. Distr. - Surinam, Guyana. Type \& AMNH*. Ref. - Doesburg, 1966:89, fig. 62 [not 61] (habitus).

BELLULUS (Williston), 1891:1, pl. 1:1 (habitus), 1b (head) (Mixogaster). Type-locality: Mexico, Guerrero, La Venta, $300 \mathrm{ft}$. Distr. — same. Type o $\mathrm{BM}(\mathrm{NH})$. N. COMB.

BICOLoR (Walker), 1857:151 (Ceratophya). Type-locality: Brazil, Pará. Distr. - same. Type o $\mathrm{BM}(\mathrm{NH}) *$. N. COMB.

BIDENS (Fabricius), 1805:185 (Mulio). Type-locality: "America meridionali". Distr. - Guyana, Argentina. Type o" MC*. 
Biluminiferus Hull, 1944i:399. Type-locality: Brazil, Espírito Santo. Distr. - same. Type o VMNH*.

BISpiNA Hull, 1943e:707. Type-locality: Brazil, São Paulo. Distr. - same. Type or $\mathrm{BM}(\mathrm{NH})$.

BONARIENSIS Lynch Arribálzaga, 1891:194 (1892c:35), fig. 1 (wing). Typelocality: Argentina, Prov. Buenos Aires. Distr. - same. Type ? MACN.

BRUCHI Shannon, 1927d:38. Type-locality: Argentina, Córdoba, Alta Gracia. Distr. - same. Types o USNM \& MACN?.

BRUESI Hull, 1945b:77. Type-locality: Haiti, Port-au-Prince and vicinity. Distr. - same. Type $+\mathrm{MCZ}^{*}$.

BRutus Hull, 1944j:37. Type-locality: Brazil, Bahia, Igrapiúna. Distr. same. Type $0^{*} \mathrm{CU}^{*}$.

CAESAR Curran, 1940:10. Type-locálity: Brazil, Santa Catarina, Nova Teutônia. Distr. - same. Type o $\mathrm{AMNH}^{*}$.

CERIOIdes Hull, 1943e:716. Type-locality: Paraguay, San Bernardino. Distr. - same. Type o BM(NH).

CHRYsidimimus (Hull), 1937c:116 (Chysidimyia). Type-locality: Brazil, Santarém. Distr. - same. Type o CM. Ref. - Hull, 1938:128, fig. 7 (head).

chrysidiformis, Hull, 1944e:241, misspelling.

CLARIPENNIS (Hine), 1914:334 (Mixogaster). Type-locality: Guatemala, Los Amates. Distr. - same. Type o OhSU. N. COMB.

COARCtatus Loew, 1864a:74 (1864b:236): Type-locality: U.S.A., District of Columbia. Distr. - U.S.A. (Nebraska to Maryland, south to Florida); Mexico. Types of o MCZ (lost - FCT). Ref. - Curran, 1925a:86 (redescription), pl. 5:46 (scutellum \& antenna).

CoNops Curran, 1940:4. Type-locality: Brazil, Mato Grosso, Maracaju. Distr. - same. Type o AMNH*.

Corona Curran, 1940:9. Type-locality: Brazil, Santa Catarina, Nova Teutônia. Distr. - same. Type o AMNH*.

CRASSITARSIS (Macquart), 1848:198 (38) (Aphritis). Type-locality: "Río Negro". Distr. - same. Types ox OX*.

CURRANI Goot, 1964:214 (nom. nov. for clavicornis Curran). Distr. Guyana.

clavicornis Curran, 1940:6 (preocc. Sack, 1926). Type-locality: Guyana, Mazaruni, High Forest. Type o $\mathrm{BM}(\mathrm{NH})$.

CYANEIVENTRIS (Macquart), 1846:249 (121), pl. 10, fig. 14 (wing) (Aphritis). Type-locality: "Colombia". Distr. - same. Types † OXF*.

cyanoventris, Williston, 1886:310, misspelling.

cyaneus Perty, 1930:186, pl. 37:4. Type-locality: Brazil, "Provincia Piauhiensis". Distr. - same. Type(s) ? ZSM.

DIAPHANUS Sack, 1921:146, figs. 21 (habitus), 22 (head). Type-locality: Paraguay, St. Trinidad. Distr. - Brazil, Paraguay. Type o DEI.

EMERALDA Hull, 1943e:719. Type-locality: "Guyana". Distr. - same. Type o $\mathrm{BM}(\mathrm{NH})$.

EUTRISTIS Curran, 1925a:74, pl. 4:35 (head). Type-locality: Mexico, Yucatán. Distr. - same. Type o U.K.

Falcatus Williston, 1887b:9. Type-locality: Mexico, Isthmus of Tehuantepec. Distr. - same. Types o USNM.

Fenestratus Hull, 1943e:712. Type-locality: “Amazon". Distr. - same. Type o $\mathrm{BM}(\mathrm{NH})$.

Flavipennis Curran, 1925c:342. Type-locality: Guyana, Bartica, Distr. same. Type o MCZ. 
Flavofascium Curran, 1925c:346. Type-locality: Brazil, Minas Gerais, Lassance. Distr. - same. Type o $\mathrm{CU}^{*}$.

Flavoluna Hull, 1943e:718. Type-locality: Paraguay, San Bernardino. Distr. - same. Type o $\mathrm{BM}(\mathrm{NH})$.

Flavomarginatus Curran, 1925b:245. Type-locality: Peru, Putamayo. Distr. - Brazil (Amapá), Peru. Type ơ CU*.

FUCATISsimus (Hull), 1937c:120" (Syrphipogon). Type-locality: "South America". Distr. - same. Type o CM. Ref. - Hull, 1938:128, fig. 6 (head).

Fulgens Wiedemann, 1830:82. Type-locality: U.S.A., Georgia [as "New Georgia"]. Distr. - U.S.A. (New Jersey south to Florida); Guyana. Type \& ZMB. Ref. - Curran, 1925a:50 (redescription), pl. $3: 17$ (habitus).

Euglossoides Gray, 1832:779, pl. 125:2. Type-locality: ??? Type ? OXF? GAIGEI Steyskal, 1953:1, figs. 1a (wing tip), b (antenna), c (scutellum), d (head). Type-locality: Panama, Chiriquí Prov., Progreso. Distr. - Panama. Type o MZM.

GEIJSKESI Doesburg, 1966:80. Type-locality: Surinam, near Paramaribo, Kwatta, parwa-wood. Distr. - Surinam. Type o Doesburg.

Gracilis Bigot, 1883b:320. Type-locality: "Mexico". Distr. - same. Type o $\mathrm{BM}(\mathrm{NH}) *$.

GRANulatus Curran, 1940:9. Type-locality: Guyana, Mazaruni. Distr. Guyana. Type o $\mathrm{BM}(\mathrm{NH})$.

Hermetia Curran, 1936b:3. Type-locality: Panama, Canal Zone, Barro Colorado Island. Distr. - same. Type o AMNH*.

HERMETOIDES Curran, 1940:8. Type-locality: Guyana, Essequibo River, Moraballi Creek. Distr. - same. Type or BM(NH).

HISTRIo Wiedemann, 1830:83, pl. 9, fig. 6 (abdmen). Type-locality: Uruguay, Montevideo. Distr. - same. Type of ZMB.

HoNDuranius Hull, 1940a:247. Type-locality: "Honduras, Salada River, 15 miles inland from Ceiba". Distr. - same. Type o Hull.

IGNOBILIS (Rondani), 1848:73 (Aphritis). Type-locality: "Brazil". Distr. same. Types ơ $\sigma^{7}$ MIZUN.

IHERINGI Bezzi, 1910:320. Type-locality: Brazil, State of São Paulo. Distr. - same. Type o MCSNM*.

INAEQUALIS Loew, 1866b:40 (1872b:100). Type-locality: "Cuba", Distr. same. Type ơ MCZ (lost)*.

INSTABILIS Wiedemann, 1830:83. Type-locality: "Brazil". Distr. - Brazil, Argentina. Type o ZMIB.

dives Rondani, 1848:72. (Aphritis), Type-locality: "Brazil". Type of UTOR.

LAEtus Loew, 1864a:74 (1864b:236). Type-locality "Cuba". Distr. - U.S.A. (Maryland south to Texas, Florida); Antilles. Types MCZ (lost)*.

LANGI Curran, 1925c:341. Type-locality: Guyana, Kamakusa. Distr. Guyana, Brazil. Type o* CU*.

LAzULI (Hull), 1944e:241 (Chrysidimyia). Type-Iocality: "Amazons, Vila Nova". Distr. - same. Type ₹ BM (NH).

Limbatus Wiedemann, 1830:85. Type-locality: Brazil, Rio Grande do Sul, Caçapava do Sul. Distr. - same. Type ? ZMB.

LURIDESCENS (Walker), 1857:151 (Ceratophya). Type-locality: "Valley of Amazon". Distr. - same. Type \& BM(NH)*. N. COMB.

MACQUARTII Lynch Arribálzaga, 1891:126 (1892c:30) (nom. nov, for angustus Macquart, 1848). Distr. - Uruguay.

angustus Macquart, 1848:198 (38) (Aphritis; preocc. Macquart, 1846). Type-locality: "Minas". Types on क MNHN?

angustatus, Fluke, 1957:29, misspelling. 
MARCELI Curran, 1936b:7. Type-locality: Brazil, Mato Grosso, Chapada Distr. - same. Type o $\mathrm{AMNH}^{*}$.

MellogutTa Hull, 1943e:704. Type-locality: "Amazon". Distr. - same. Type o $\mathrm{BM}(\mathrm{NH})$.

MIRABILIS Williston, 1888:257. Type-locality: Brazil, Mato Grosso, Chapada. Distr. - Brazil (Mato Grosso, Santa Catarina), Paraguay. Types o $\sigma^{*}$ AMNH*.

bertonii Bezzi, 1910:319. Type-locality: Paraguay, Puerto Bertoni. Types ot o MCSNM. N. SYN.

arcuata Curran, 1941:250. Type-locality: Brazil, Santa Catarina, Nova Teutônia. Type ơ AMNH*. N. SYN.

Miris Curran, 1940:7. Type-locality: Brazil, Rio de Janeiro. Distr. Brazil (Rio de Janeiro, Santa Catarina). Type o" AMNH*.

Mus Curran, 1936b:5. Type-locality: Brazil, São Paulo, Juquiá. Distr. same. Type o AMNH*.

NERo Curran, 1936b:6. Type-locality: Brazil, São Paulo. Distr. - Brazil (São Paulo, Santa Catarina). Type o AMNH*.

NESTOR Curran, 1940:11. Type-locality: Brazil, Santa Catarina, Nova Teutônia. Distr. - same. Type ơ AMNH*.

NIGER Williston, 1891:4, pl. 1:3 (habitus), 3a (head). Type-locality: Mexico, Vera Paz, Panima. Distr. - Mexico, Bolivia. Type of BM(NH).

manni Shannon, 1923b:80. Type-locality: Bolivia, Ivon, Río Beni. Type \& USNM*. N. SYN.

NIGRISPINosus Shannon, 1927a:21. Type-locality: Brazil, Amazonas, Tefé (as Ega). Distr. - Panama, Brazil. Type o $\mathrm{BM}(\mathrm{NH})$.

NORMALIS Curran, 1925c:343. Type-locality: Guyana, West bank of Demerara River. Distr. - Guyana. Type \& $\mathrm{AMNH}^{*}$.

oligonax Hull, 1944j:35. Type-locality: Ecuador, Pto. America, Río Putumayo. Distr. - Ecuador, Brazil. Type o CU*.

opulentus Bigot, 1883b:319. Type-locality: "Brazil". Distr. - same. Type o $\mathrm{BM}(\mathrm{NH})$.

PAUPER (Rondani) 1848:74 (Aphritis). Type-locality: "Brazil". Distr. same. Type $0^{*}$ MIZUN.

PUlcher Williston, 1887b:5. Type-locality: Cuba, San Domingo. Distr. Mexico (Durango), Cuba. Type q USNM*.

REMotus Knab, 1917:142. Type-locality: Cuba. Distr. - same. Type o USNM*.

REMUS Curran, 1941:250. Type-locality: Brazil, Santa Catarina, Nova Teutônia. Distr. — same. Type o AMNH*.

RHEOCHRYsSUS Hull, 1944j:38. Type-locality: Argentina, Jujuy, S. Juancito. Distr. - Brazil (São Paulo), Argentina (Jujuy). Type o CU*.

RUBRIVENTRIS Lynch Arribálzaga, 1891:128 (1892c:32), fig. 2 (head). Typelocality: Argentina, Buenos Aires, Chacabuco, Distr. - same. Type ? MACN.

RUFiventRIS (Rondani), 1848:73 (Aphritis). Type-locality: "Brazil". Distr. same. Type o MIZUN.

SEABRaI (Papavero), 1962:317, fig. 1 (head) (Pseudomicrodon). Type-locality: Brazil, Guanabara, Sumaré. Type ơ Seabra. N. COMB.

SHannoni Curran, 1940:8. Type-locality: Brazil, Mato Grosso, Maracaju. Distr. - same. Type o AMNH*.

SOLITARIUS Curran, 1930f:8. Type-locality: Panama, Canal Zone, Barro Colorado Island. Distr. - same. Type of $\mathrm{AMNH}^{*}$.

SPLendens Wiedemann, 1830:84. Type-locality: "Brazil". Distr. - Brazil (Mato Grosso, Santa Catarina). Type o VMNH*. 
Stramineus Hull, 1943e:703. Type-locality: Brazil, Pará. Distr. - same. Type o $\mathrm{BM}(\mathrm{NH})$.

SUPERBUS Wiedemann, 1830:82. Type-locality: "Brazil". Distr. - same. Type + SMF*.

TIGRINUS Curran, 1940:11. Type-locality: Brazil, Rio de Janeiro. Distr. same. Type o AMNH*.

TRILINEA Hull, 1943e:710. Type-locality: "Amazon". Distr. — same. Type o $\mathrm{BM}(\mathrm{NH})$.

TRIVITTATUS Curran, 1925c:344. Type-locality: Guyana, Kartabo. Distr. Guyana, Brazil (Amapá). Type ơ AMNH*.

violaceus (Macquart), 1842:73 (13), pl. 1, figs. 3 (habitus), 3a (head) (Aphritis). Type-locality: "Chile". Distr. - Chile (Coquimbo Malleco), Type $\sigma^{*}$ MNHN*.

vIOLENS Townsend, 1895a:34. Type-locality: "Jamaica". Distr, - same. Type + ? UK.

virgo Curran, 1940:7. Type-locality: Brazil, Santa Catarina, Nova Teutônia. Distr. - same. Type o AMNH*.

viridis Townsend, 1895b:610. Type-locality: Mexico, Baja California, San José del Cabo. Distr. - Canada (British Columbia), U.S.A. (California); Mexico. Type \& CAS (destroyed).

WILLISTONI Mik, 1899:143 (nom. nov. for inermis Williston). Distr. Argentina.

inermis Williston, 1888:258 (preocc. Loew, 1858). Type-locality: Argentina, Entre Ríos. Type o" AMNH*.

\section{Genus Nothomicrodon Wheeler}

NOTHOMICRODON Wheeler, 1924:243. Type-species, aztecarum Wheeler (orig. des.).

AZTECARUM Wheeler, 1924:243, fig. 2 (habitus), 3 (details of puparium). Type-locality: Panama, Canal Zone, Frijoles. Types (larvae) USNM*.

\section{Subfamily MILESIINAE \\ Tribe PIPIZINI}

\section{Genus Pipiza Fallén}

PIPIZA Fallén, 1810:11. Type-species, Musca noctiluca Linnaeus (Curtis, 1837:pl. 669).

Penium Philippi, 1865:741. Type-species, triste Philippi (mon.). Ref. - Shannon \& Aubertin, 1933 (key).

AURANTTPES (Bigot), 1857a:297, pl. 7:8 (wing, head, antenna) (Cheilosia). Type-locality: "Chile". Distr. - Chile. Type o" OXF*.

flavipes Philippi, 1865:738 (Sterphus; preocc. Philippi, 1865). Typelocality: "Chile". Type(s) ? MNHNS.

?BELlula Williston, 1891:6. Type-locality: Mexico, Guerrero, Xucumanatlán, $7000 \mathrm{ft}$. Distr. - same. Types ơ BM(NH).

CLARIPENNIS Shannon \& Aubertin, 1933:144. Type-locality: Chile, Casa Pangue. Distr. - Chile, Argentina (Neuquén). Type o $\mathrm{BM}(\mathrm{NH})$.

Flavipes (Philippi), 1865:pl. 27, fig. 41 (Penium). Type-locality: "Chile". Distr. - same. Type(s) \& MNHNS [as 1st revisers, we here recognize Penium flavipes as senior to Sterphus flavipes Philippi]. 
?HIRsutops Hull, 1949c:744. Type-locality: Mexico, Oaxaca. Distr. - same. Type o $\mathrm{BM}(\mathrm{NH})$.

TRISTIS (Philippi), 1865:741 (Penium). Type-locality: Chile, Corral. Distr. - Chile. Type(s) ? MNHNS.

dubium Bigot, 1884a:557 (Penium). Type-locality: "Chile". Types of $\mathrm{BM}(\mathrm{NH})$.

\section{Genus TRICHOPSOMYia Williston}

TRICHOPSOMYIA Williston, 1888:259. Type-species, polita Williston (Hull, 1949d:330).

Halictomyia Shannon, 1927a:13. Type-species, boliviensis Shannon (orig. des.).

Ref. - Fluke, 1937 (key).

Boliviensis (Shannon), 1927a:13, fig. 6 (wing) (Halictomyia). Type-locality: Bolivia, Cavinas, Beni. Distr. - same. Type \& USNM.

CATHARINENSIS (Enderlein), 1938:195 (Pipiza). Type-locality: Brazil, Santa Catarina, Hochland. Type \& ZMB. N. COMB.

CURRANI Fluke, 1937:2, fig. 1 (abdomen). Type-locality: Brazil, Santa Catarina, Nova Teutônia. Distr. - same. Type o AMNH*.

Granditibialis Fluke, 1937:3, figs. 4 (hind leg), 5 (antenna). Type-locality: Brazil, Santa Catarina, Nova Teutônia. Distr. - same. Type $q$ AMNH*.

LASIOTIBIALIS Fluke, 1937:2, figs. 2 (head), 3 (antenna), Type-locality: Brazil, Santa Catarina, Nova Teutônia. Distr. - same. Type ơ AMNH*.

LONGICORNIS Williston, 1888:261. Type-locality: Brazil, Mato Grosso, Chapada. Distr. - same. Type of AMNH*.

Polita Williston, 1888:260. Type-locality: Brazil, Mato Grosso, Chapada. Distr. - same. Types o \& AMNH*.

Puella 'Williston, 1888:260. Type-locality: Brazil, Mato Grosso, Chapada. Distr. - same. Types o o AMNH*.

TUBerculata Williston, 1888:260. Type-locality: Brazil, Mato Grosso, Chapada. Distr. - same. Type \& AMNH*,

Uleana (Enderlein), 1938:195 (Pipiza). Type-locality: Brazil, Santa Catarina, Hochland. Distr. - same. Type o ZMB. N. COMB.

Urania Hull, 1949a:73. Type-locality: Peru, Pucallpa. Distr. - same. Type o Hull.

\section{Tribe CHEILOSIINI}

\section{Genus Cheilosia Meigen}

CHEIlosta Meigen, 1822:296. Type-species, Eristalis scutellatus Fallén (Rondani, 1856:51).

Chilosia, auctorum, misspelling or emendation.

Ref. - Hull \& Fluke, 1950 (rev. of subgroups).

\section{Subgenus Cheilosia Meigen}

Ref. - Hull \& Fluke, 1950 (rev., key).

?AURotecta Giglio-Tos, 1892d:4. Type-locality: Mexico, Orizaba. Distr. same. Types of of UTOR. Ref. - Giglio-Tos, 1893: fig. 22 (head). auritecta, Fluke, 1957:44, misspelling.

CHRYSOCHLAMYS Williston, 1891:8, pl. 1:4 (habitus), 4a (head) (Chilosia). Type-locality: Mexico, Guerrero, Omiltemi; Sierra de las Aguas Escondidas, 9500 ft. Distr. - U.S.A. (California), Mexico. 
Types o ㅇ $\mathrm{BM}(\mathrm{NH})$ \& $\mathrm{AMNH}$ * Ref. - Hull \& Fluke, 1950:346 (redescription), fig. 35 (head).

SORORIA Williston, 1891:8 (Chilosia). Type-locality: Mexico, Durango, Ciudad. Distr. - same. Types o BM(NH). Ref. - Hull \& Fluke, 1950: 348 (redescription), fig. 24 (head).

\section{Subgenus Cartosyrphus Bigot}

CARTOSYRPHUS Bigot, 1883b:230 (as genus). Type-species, Syrphus paganus Meigen (Coquillett, 1910b:376). Ref. - Fluke \& Hull, 1947 (rev., key).

FRoNTOSA (Bigot), 1884a:553 (Cartosyrphus). Type-locality: "Mexico". Distr. - same. Type o $\mathrm{BM}(\mathrm{NH})$.

SORoRCula Williston, 1891:9 (Chilosia). Type-locality: Mexico, Guerrero: Omiltemi, $8000 \mathrm{ft}$. , Xucumanatlán, $7000 \mathrm{ft}$.; Sierra de las Aguas Escondidas, $7000 \mathrm{ft}$. Distr. - U.S.A. (Oregon to Idaho), south to Mexico. Types o BM(NH). Ref. - Fluke \& Hull, 1947:250, fig. 33 (head) (redescription).

\section{Genus RHINGIA Scopoli}

RHINGIA Scopoli, 1763:358. Type-species: Conops rostrata Linnaeus (mon.). LONGIROSTRIS Fluke, 1943:430, figs. 10 (wing tip), 11 (head). Type-locality: Ecuador, Bolivar, Hda. Talahua, $3100 \mathrm{ft}$. Distr. - same. Type o $\mathrm{AMNH}^{*}$.

NIGRA Macquart, 1846:261 (133), tab. 11:10 (head). Type-locality: "Colombia". Distr. - Mexico (Tabasco), Colombia, Brazil. Types o OXF*. Ref. - Fluke, 1943:427, fig. 12 (head).

harrisi Curran, 1925b:253. Type-locality: Brazil, Rio de Janeiro, Petrópolis. Type ơ CU. N. SYN.

\section{Genus Chamaesyrphus Mik}

CHAMAESYRPHUS Mik, 1895:133. Type-species, Rhingia scaevoides Fallén (mon.).

WILlistonI (Snow), 1895a:187 (Pelecocera). Type-locality: U.S.A., New Mexico. Distr. - U.S.A. (New Mexico), Mexico. Type o UK. skinneri Johnson, 1903:101 (Chilosia). Type-locality: U.S.A., New Mexico, Beulah. Type o ANSP. N. SYN.

\section{Tribe VOLUCELLINI}

\section{Genus Ornidia Lepeletier \& Serville}

ORNIDIA Lepeletier \& Serville, in Latreille et al., 1828:786. Type-species, Syrphus obesus Fabricius (orig. des.). Refs. - Curran, 1930d (key); Val, 1972 (key, biometry, evolution).

AEMula (Willison), 1888:272 (Volucella). Type-locality: Bolivia, Santa Cruz, Piedra Blanca. [4 $\mathrm{km}$ West of Corumbá, Mato Grosso, Brazil]. Distr. - Venezuela, Bolivia, Colombia, Brazil. Types o AMNH*.

MAJOR Curran, 1930d:2. Type-locality: "Brazil". Distr. - Costa Rica, Panama, Ecuador, Peru, Brazil. Type o AMNH*.

OBESA (Fabricius), 1775:763 (Syrphus). Type-locality: "America". Distr. U.S.A. (Georgia, New Mexico, Texas, Florida); south to Peru 
and Argentina (Entre Rios, Tucumán). Types ? Kiel [not found -JRV]. Ref. - Williston, 1887b:312 (redescription), pl. 5:9 (head); Sack, 1921:138 (description), figs, 12 (larva), 11e (spiracular horn), 13 (anal organ of larva), 14 (puparium); Curran, 1925a: 206, pl. 10:141 (male genitalia).

violacea Macquart, 1842:83 (23) (Volucella; preocc. Lepeletier \& Serville, 1828; Say, 1830). Type-locality: Brazil. Type of MNHN.

obesoides Giglio-Tos, 1892c:4 (Volucella). Type-locality: Mexico. Type o* UTOR*. N. SYN.

\section{Genus Tachinosyrphus Hull}

TACHINOSYRPHUS Hull, 1936b:167. Type-species, pseudotachina Hull (orig. des.).

PSEUdotachina Hull, 1936b:167. Type-locality: Peru, Crucero, Moscardones. Distr. - same. Type o USNM*. Ref. - Hull, 1949d:349, fig. $20 \mathrm{f}$ (head).

\section{Genus Copestrlum Macquart}

COPESTYLUM Macquart, 1846:252 (124). Type-species, flaviventris Macquart (mon.) $=$ marginatum Say.

Phalacromya Rondani, 1848:67. Type-species, submetallica Rondani (Coquillett, 1910a:587).

Phalacromyia, emendation.

Glaurotricha Thomson, 1869:493. Type-species, muscaria Thomson (mon.).

Haurotricha Thomson, 1869:493. Incorrect original spelling of Glaurotricha Thomson (1st reviser, Bigot, 1883b:250).

Atemnocera Bigot, 1882a:cxiv. 'Type-species, Volucella scutellata Macquart (mon.).

Apophysophora Williston, 1888:276. Type-species, scutellata Williston (Curran, 1930d:5) = trituberculatum Thompson.

Megametopon Giglio-Tos, 1891:5. Type-species, Ophromyia nasica Williston (Williston, 1892:79 \& Giglio-Tos, 1892e:44).

Ophromyia Williston, 1891:55. Type-species, nasica Williston (mon.).

Camerania Giglio-Tos, 1892a:3. Type-species, macrocephada Giglio-Tos (as Temnocera megacephala? Loew; orig. des.).

Viereckomyia Curran, 1925b:246. Type-species, Temnocera gibbera Schiner (orig. des.).

Lepidopsis Curran, 1925b:247. Type-species, compactus Curran (orig. des.). Lepidosis, Thompson, 1965, misspelling.

Volucella, subg. Volosyrpha Shannon, 1929:575. Type-species, tibialis Shannon (orig. des.) = rufitarse Thompson.

Volucellosia Curran, 1930d:5. Type-specie, Volucella fornax Townsend (orig. des.).

Refs. - Curran, 1930d (keys); Curran, 1939a (key); Curran, 1947b (key to vesicularium group); Curran, 1953 (keys to abdominale and esuriens groups); Curran, 1935 (key to marginatum group); Fluke, 1953 (key to scutellatum group).

ABdominale (Wiedemann) 1830:196 (Volucella). Type-locality: "Cuba". Distr. - U.S.A. (Florida); Bahamas (Grand Turk I.), Cuba. Types o VMNH \& ZMB. Ref. - Curran, 1926a: 64 (redescription). N. COMB.

ABRUPTA (Curran), 1925b:251 (Phalacromyia). Type-locality: Brazil, Rio Grande do Norte, Baixa Verde. Distr. - Venezuela, Brazil. 
Type o CU. Ref. - Fluke, 1951b 22 (redescription), figs. 10 (head), 19 (wing), 34 (scutellum). 44 (antenna). N. COMB.

ACUTIFrons (Curran), 1939b:12 (Volucella). Type-locality: Peru, Tarapoto Region. Distr. - Peru. Type o $\mathrm{AMNH}^{*}$. N. COMB.

ALBIFrons (Curran), 1939b:16 (Volucella). Type-locality: Brazil, Santa Catarina, Nova Teutônia. Distr. - same. Type o ${ }^{*}$ AMNH*. N. COMB.

albipilosum (Hull), 1937a:166 (Volucella). Type-locality: Mexico, San Martin. Distr. - same. Type o USNM*. N. COMB.

ALBITARSE (Lynch Arribálzaga), 1892b:244 (1892c:145) (Phalacromyia). Type-locality: Argentina, Altas Misiones. Distr. - same. Type of MACN. N. COMB.

ALCEDO (Curran), 1926a:53; 1934b:388 (Volucella). Type-locality: "Venezuela". Distr. - Guyana, Venezuela. Type + MCZ*. N. COMB.

ALCEDoIdes (Curran), 1939b:11 (Volucella). Type-locality: Trinidad, St. Ann's. Distr. - same. Type o AMNH*. N. COMB.

ALCHIMISTA (Rondani), 1848:64, fig. 2 (wing) (Volucella). Type-locality: "Brazil". Distr. - same. Type o MIZUN. N. COMB.

AMBRosetTil (Lynch Arribálzaga), 1892d:179 (1892c:129) (Volucella). Type-locality: Argentina, Misiones, Moconá. Distr. — same. Types o + MACN. N. COMB.

AMETHYSTINUM (Bigot), 1875:479 (Volucella, as amethistina; emendation: Williston, 1891:52). Type-locality: "Mexico". Distr. _ same. Type o OXF* $^{*}$. N. COMB.

ANNA (Williston), 1887b:138, pl. 6:8 (head) (Volucella). Type-locality: U.S.A., Arizona. Distr. - U.S.A. (Arizona, New Mexico); Mexico (Chiapas). Type o USNM*. Ref. - Curran, 1925a:206, pl. 10:142 (male genitalia). N. COMB.

APERTUM (Walker), 1860:292 (Volucella). Type-locality: "Mexico". Distr. - same. Type $\sigma^{*} \mathrm{BM}(\mathrm{NH})$. N. COMB.

APICAle (Loew), 1866a:151 (1872b:25) (Volucella). Type-locality: "Cuba". Distr. - U.S.A. (Maryland [Wirth, et al., 1965:600]); Cuba. Type o MCZ. Ref. - Williston, 1887b:149 (redescription). N. COMB.

APICIFERUM (Townsend), 1895a:40 (Volucella). Type-locality: U.S.A., New Mexico, Las Cruces. Distr. - U.S.A. (California to Arizona); Mexico. Type o UK. N. COMB.

APICUla (Curran), 1939b:6 (Volucella). Type-locality: Peru, Middle Río Ucayali. Distr. - Panama, Brazil (Acre), Peru. Type o AMNH*. ARICA (Fluke), 1950b:450, figs. 14 (head), 15 (antenna), 16 (male genitalia) (Volucell $\alpha$ ). Type-locality: Chile, Arica, Putre, $3650 \mathrm{~m}$. Distr. - Chile (Antofagasta, Tarapacá). Type of UCSC. N. COMB. APICIA (Curran), 1930d:22 (Volucella). Type-locality: Argentina, foothills west of Mendoza. Distr. - Argentina (Tucumán to Rio Negro). Type o AMNH. N. COMB.

diversum Fluke, 1951b:11, figs. 3 (head), 25 (scutellum), 38 (antenna), 52 (male genitalia) (Volucella). Type-locality: Argentina, Río Negro, Isla Choele Choel. Type $\sigma^{*}$ IML. N. SYN.

astarte (Hull, 1950a:70 (Volucella). Type-locality: Peru, Pucallpa. Distr. - same. Type o Hull. N. COMB.

ASTER (Curran), 1939b:3 (Volucella). Type-locality: Peru, Piches and Perené Valleys, 2000-3000 ft. Distr. - same. Type o AMNH*. N. COMB.

AUREUM (Fluke), 1951b:13, figs, 6 (head), 27 (scutellum) (Volucella). Type-locality: Colombia, Cundinamarca, Zipacoa. Distr. - same. Type o AMNH*. N. COMB. 
AVIDUm (Osten-Sacken), 1877:333 (Volucella). Type-locality: U.S.A., California. Distr. - Canada (British Columbia) south to U.S.A. (California \& Texas); Mexico (Guanajuato). Types $0^{*} \mathrm{MCZ}^{*}$. Ref. - Williston, 1887b:139 (redescription). N. COMB.

AZUREUm (Philippi), 1865:734 (Volucella). Type-locality: Chile, Colchagua, "Prope Llico". Distr. - Chile (Aconcagua - Linares), Argentina. Type o MNHNS. N. COMB.

AZURINUM (Hull), 1941h:314 (Volucella). Type-locality: Brazil, Bonito(?). Distr. - same. Type or Hull. N. COMB.

BASSLERI (Curran), 1939b:9 (Volucella). Type-locality: Peru, Iquitos. Distr. - same. Type o AMNH*. N. COMB.

BEATRICEA (Hull), 1950c:235 (Volucella). Type-locality: "Ecuador". Distr. - Ecuador. Type o Hull. N. COMB.

ecuadorea Fluke, 1951b:14, figs. 7 (head), 28 (scutellum), 51 (male genitalia) (Volucella). Type-locality: Ecuador, Azuay, Cuenca, $2650 \mathrm{~m}$. Type o AMNH*.

BL.LINDA (Hull), 1949h:31 (Volucella). Type-locality: Brazil, Santa Catarina, Nova Teutônia. Distr. - same. Type ot Hull. N. COMB.

BELlulum (Williston), 1891:42 (Phalacromyia). Type-locality: Mexico, North Yucatán, Temax. Distr. - same. Types $\sigma^{\circ} \mathrm{BM}(\mathrm{NH})$. N. COMB.

Bequaerti Curran, 1930f:2. Type-locality: Mexico, Yucatán, Chichén Itzá. Distr. - same. Type o AMNH*.

BIMACUlATUM (Sack), 1941:110 (1951:106) (Phalacromyia). Type-locality: Peru, Pichis and Pachitea. Distr. - same. Types $c+$ MD. N. COMB.

BINOMINATUM (Goot), 1964:214 (Volucella; nom. nov. for fuscipenne Macquart). Distr. - Bolivia. N. COMB.

fuscipenne Macquart, 1850:435 (131), pl. 12, fig. 14 (head) (Volucella; preocc. Macquart, 1842). Type-locality: Bolivia, Chiquitos. Type $q$ MNHN. N. COMB.

BIPUNCTATUM (Hull), 1941g:157 (Phalacromyia). Type-locality: Paraguay, Pastorea. Distr. - same. Type o MCZ*. N. COMB.

Bolivianum (Hine), 1914:338 (Volucella). Type-locality: Bolivia, La Paz. Distr. - Bolivia, Peru. Type \& OhSU. N. COMB.

BRADLEYI (Curran), 1925c:348 (Volucella). Type-locality: Peru, Matucana. Distr. - Chile (Tarapacá), Peru (Lima, Arequipa). Type † CU. Ref. - Fluke, 1951b:26 (redescription). N. COMB.

BRAzILIANUM (Hull), 1938:124, fig. 4 (head) (Volucella). Type-locality: Brazil, Amazonas, Rio Purus, Huitanaã. Distr. - same. Type o CM. N. COMB.

BReve (Giglio-Tos), 1892c:4 (Volucella). Type-locality: Mexico, Meztillán. Distr. - same. Types $0^{*}$ \& UTOR. N. COMB.

BREvifacies (Curran), 1926a:52; 1934b:386, fig. 29 (head) (Volucella). Type-locality: Guyana, Kartabo. Distr. - Guyana, Brazil (Santa Catarina), Argentina (Tucumán). Type o AMNH*. N. COMB.

BRevivitTatum (Curran), 1930d:14 (Volucella). Type-locality: Brazil, Mato Grosso), Argentina (Misiones). Type o AMNH* N. COMB.

BRUNERI (Curran), 1939b:6 (Volucella). Type-locality: Cuba, Oriente, Sierra del Cobre, Loma del Gato. Distr. - same. Type o AMNH*. N. COMB.

BIUUNNICOLOR (Hull), 1938:123, figs. 8 (head), 9 (abdomen) (Volucella). Type-locality: Brazil, Pará, Santarém. Distr. - same. Type ơ CM. N. COMB.

BRUNNIGASTER (Hull), 1943k:41 (Volucella). Type-locality: Colombia, Meta District. Distr. - same. Type o USNM. N. COMB. 
BULboSUM (Fluke) 1951b:24, figs. 13 (head), 35 (scutellum), 45 (antenna), 63 (male genitalia) (Volucella). Type-locality: Ecuador, Tungurahua, Volcano Tungurahua, Minza Chica, $3750 \mathrm{~m}$. Distr. - Ecuador, Argentina (Catamarca, Tucumán). Type o AMNH*. N. COMB. CAEsariatum (Williston), 1891:49 (Volucella). Type-locality: Mexico, Orizaba. Distr. - U.S.A. (Arizona), Mexico. Type \& $\mathrm{BM}(\mathrm{NH})$. N. COMB.

coesariata, Giglio-Tos, 1892, misspelling.

hirsuta Giglio-Tos, 1892c:3 (Volucella). Type-locality: Mexico, Meztillán. Type $0^{*}$ UTOR.

Calcchaetum (Hull), 1941h:313 (Volucella). Type-locality: "Brazil". Distr. - same. Type o Hull. N. COMB.

CAMPosi (Curran), 1939b:8 (Volucella). Type-locality: Ecuador, Isla Puná, Puerto Grande. Distr. - Ecuador, Argentina (Tucumán). Type o $A M N H *$. N. COMB.

CHAETOGASTER (Hull), 1943d:22 (Volucella). Type-locality: Brazil, Amazon. Distr. - same. Type o BM(NH). Ref. - Hull, 1949d:349, fig. 20h (antenna), 20i (head). N. COMB.

CHAETOPHORUM (Williston), 1887b:149 (Volucella). Type-locality: Mexico, Isthmus of Tehuantepec. Distr. - Mexico. Types $0^{\circ}$ i USNM. Ref. - Williston, 1892: pl. 1:15 (habitus), 15a (head), 15b (antenna). N. COMB.

Chalybescens (Wiedemann), 1830:204 (Volucella). Type-locality: "Brazil". Distr. - Cuba, Trinidad, Colombia, Paraguay, Brazil. Type(s) o VMNH [also 30, SMF*, syntypes?]. Ref. - Curran, 1926a:59 (redescription). N. COMB.

ChapAdense (Curran), 1930d:13 (Volucella). Type-locality: Brazil, Mato Grosso, Chapada. Distr. - Brazil (Mato Grosso), Peru, Argentina (Tucumán). Type of AMNH*. N. COMB.

Chlorosum (Hull), 1930:148 (Volucella). Type-locality: "Mexico". Distr. same. Type o ANSP. N. COMB.

cinctiventre (Curran), 1930d:16 (Volucella). Type-locality: Panama, Canal Zone, Barro Colorado Island. Distr. - same. Type o AMNH*. N. COMB.

CIRCE (Curran), 1939b:1 (Volucella). Type-locality: Brazil, Santa Catarina, Nova Teutônia. Distr. - same. Type ơ AMNH*. N. COMB.

CIRCUMDATUM (Walker), 1857:154 (Temnocera). Type-locality: "Valley of Amazon". Distr. - same. Type ? BM(NH). N. COMB.

CIRCUMSCRIPTUM (Curran), 1939b:13 (Volucella). Type-locality: Brazil, São Paulo. Distr. - same. Type AMNH*. N. COMB.

Cr.ARIPENNE (Curran), 1925b:247 (Phalacromyia). Type-locality: Peru, Lima. Distr. - same. Type o CU. N. COMB.

ClARUM (Hull), 1942h:22 (Volucella). Type-locality: Brazil, São Paulo, Ipiranga. Distr. - same. Type of Hull. N. COMB.

Cockerelry (Curran), 1927c:85 (Volucella). Type-locality: Peru, Tingo Maria. Distr. - Chile, Peru. Type o AMNH*. Ref, - Fluke, 1951b:17 (description), figs. 46 (antenna), 60 (male genitalia). N. COMB.

colombiense Thompson (nom. nov. for bequaerti Curran, 1936). Distr. Mexico, Guatemala. NOM. NOV.

bequaerti Curran, 1936b:9 (Volucella; preocc. Curran, 1930). Typelocality: Guatemala, Guatalón, Moca, $3000 \mathrm{ft}$. Type o AMNH*.

Compactum (Curran), 1925b:247 (Lepidopsis). Type-locality: Brazil, Rio de Janeiro. Distr. - Brazil (Rio de Janeiro, Santa Catarina). Type o CU. N. COMB. 
COMSTOCKI (Williston), 1887b:138, pl. 6:9 (head) (Volucella). Type-locality: U.S.A., Arizona \& New Mexico. Distr. - U.S.A. (Utah to Wyoming, south to Arizona \& Texas); Mexico (Sonora). Types $0^{\pi}$ \& USNM. N. COMB.

Concinnum (Philippi), 1865:733 (Volucella). Type-locality: Chile, Prov. Santiago \& Prov. Aconcagua. Distr. - Chile (Coquimbo - Santiago). Types ot of MNHS. N. COMB.

CONIFACIUM (Hull), 1943d:39 (Volucella). Type-locality: "Trinidad". Distr. - same. Type $\sigma^{\pi} \mathrm{BM}(\mathrm{NH})$. N. COMB.

contumax (Curran), 1939b:4 (Volucella). Type-locality: "Brazil". Distr. same. Type of AMNH*. N. COMB.

CORDIAE (Townsend), 1897a:27 (Volucella). Type-locality: Mexico, Vera Cruz, Río Nautla, San Rafael. Distr. - same. Types \& $\mathrm{BM}(\mathrm{NH})$. N. COMB.

CORRECTUM (Curran), 1927c:87 (Volucella). Type-locality: Costa Rica, San José. Distr. - Panama, Trinidad. Type o UK. N. COMB.

CORUMBENSE (Curran), 1930d:18 (Volucella). Type-locality: Brazil, Mato Grosso, Corumbá. Distr. - same. Type o AMNH*. N. COMB.

CRAVERII (Giglio-Tos), 1892c:2 (Volucella). Type-locality: "Mexico". Distr. - same. Type o UTOR. N. COMB.

CREPUSCULARIUM (Hull), 1942g:94 (Volucella). Type-locality: Brazil, Paraná, Paranaguá. Distr. - same. Type o MCZ. N. COMB.

CRUCIATUM (Hull), 1943d:37 (Volucella). Type-locality: Guatemala, Zapote. Distr. - same. Type of BM(NH). N. COMB.

Cubomaculatum (Hull), 1937a:167, fig. 5 (abdomen) (Volucella). Typelocality: Peru, Iquitos. Distr. - same. Type of USNM. N. COMB.

CUPRICOLOR (Hull), 1948b:6 (Volucella). Type-locality: "Venezuela". Distr. - same. Type $q$ USNM. N. COMB.

CURIOSUM (Curran), 1939b:1 (Volucella). Type-locality: Brazil, Santa Catarina, Nova Teutônia. Distr. - same. Type ơ AMNH*. N. COMB.

CURRANI (Fluke), 1951b:12, figs. 5 (head), 26 (scutellum), 48 (male genitalia) (Volucella). Type-locality: Ecuador, Azuay, Cuenca, $2650 \mathrm{~m}$. Distr. - Ecuador. Type o AMNH*. N. COMB.

CYANESCENS (Macquart), 1842:84 (24), pl. 6, figs. 1 (habitus), 1a (head) (Volucella). Type-locality: "Brazil". Distr. - Guyana, Brazil. Type ? MNHN? [not found - JRV]. N. COMB.

CYANOPRoctum (Curran), 1939b:11 (Volucella). Type-locality: "Peru". Type o AMNH. N. COMB.

CYANOPRORA (Curran), 1939b:10 (Volucella). Type-locality: Venezuela, Caura Valley. Distr. - same. Type o AMNH. N. COMB.

DECEPTOR (Curran), 1925b:248 (Phalacromyia). Type-locality: Venezuela, Caura Valley. Distr. - Venezuela, Brazil (Mato Grosso). Type o MCZ*. Ref. - Curran, 1930d:13, fig. 1 (head). N. COMB.

fuscipennis, Williston, 1888:276, misidentification.

Delila (Hull), 1950c:230 (Volucella). Type-locality: Peru, Chanchamayo. Distr. - same. Type o Hull. N. COMB.

DICHopTICUM Thompson (nom. nov. for violaceum Curran). Distr. - Mexico (Yucatán), Panama. NOM. NOV.

violaceum Curran, 1930f:1 (Megametopon; preocc. Say, 1830). Typelocality: Mexico, Yucatán, Chichén Itzá. Type o AMNH.

DICHroICum (Giglio-Tos), 1892c:3 (Volucella). Type-locality: Mexico, Huastec. Distr. - Mexico, Guatemala. Type + UTOR. N. COMB.

DIMORPHIUM (Curran), 1939b:14 (Volucella). Type-locality: Brazil, Santa Catarina, Nova Teutônia. Distr. - Brazil (Santa Catarina), Argentina (Tucumán). Type $\sigma^{*} \mathrm{AMNH}^{*}$. N. COMB. 
DISCALE (Curran), 1926a:57 (Volucella). Type-locality: "Grenada". Distr. - same. Type o USNM. N. COMB.

DISPAR (Macquart), 1846:251 (123), pl. 11, figs. 2 (wing), 2a (head) (Volucella). Type-locality: "New Grenada". Distr. - Guatemala, Panama, Colombia, Venezuela. Types o $0^{*} \mathrm{OXF}^{*} \&$ MNHN? N. COMB.

?metalliferum Walker p.p., 1849:636 (Volucella). Type-locality: "Venezuela". Type o BM(NH) [Curran, 1953:8 split the species without designation of lectotype; the specimen from Mexico was placed under mexicana Macquart and is considered here as valid, q.v.].

?transatlanticum Rondani, 1863:4 (Volucella). Type-locality: "America merid.". Types of o MIZUN.

DORSALE (Wiedemann), 1830:199 (Volucella). Type-locality: "Brazil". Distr. - same. Type or VMNH. Ref. - Curran, 1926a:61 (redescription). N. COMB.

DRACAENA (Curran), 1947b:2 (Volucella). Type-locality: U.S.A., Hawaii, Honolulu. Distr. - U.S.A. (Hawaii); Costa Rica (Wirth in Hardy, 1964:405). Type o AMNH*. N. COMB.

DUIDA (Curran), 1930d:21 (Volucella). Type-locality: Venezuela, Mt. Duida. Distr. - same. Type o AMNH*. N. COMB.

EMERALDA (Hull), 1934d:20 (Volucella). Type-locality: Brazil, Amazon. Distr. - same. Type o BM(NH). N. COMB.

EMILIA (Curran), 1939b:6 (Volucella). Type-locality: Guatemala, Pochuta,

Sa. Emilia, 1000 m. Distr. - same. Type o AMNH*. N. COMB.

ERINESTA (Curran), 1926a:55 (Volucella). Type-locality: Panama, Tuipo. Distr. - Guatemala, Panama. Type o AMNH*. N. COMB.

ERNestina (Curran), 1930f:5 (Volucella). Type-locality: Mexico, Yucatán, Chichén Itzá. Distr. - Mexico (Yucatån), Peru (Cuzco). Type o AMNH*. N. COMB.

ESCOMELI (Curran), 1929:489 (Volucella). Type-locality: Peru, Arequipa. Distr. - Peru, Chile (Tarapacá). Type ơ AMNH*. Ref. - Fluke, 1951b:16 (description), figs. 15 (head), 29 (scutellum), 40 (antenna), 55 (male genitalia). N. COMB.

ESURIENS (Fabricius), 1794:281 (Syrphus). Type-locality: "Americae Insulis". Distr. - Puerto Rico. Type \& Kiel*. Ref. - Curran, 1953:8 (redescription), N. COMB.

rica Curran, 1939a:6 (Volucella). Type-locality: Puerto Rico, Ensenada. Type o AMNH*.

EUGENIA (Williston), 1887b:139, pl. 6:10 (head) (Volucella). Type-locality: Bahamas; U.S.A., Florida. Distr. - U.S.A. (Florida); Bahamas. Types o USNM. N. COMB.

ingenia Curran, 1930f:4 (Volucella). Type-locality: Bahamas, Andros Island, Mangrove Cay, Gibson Cay. Type o AMNH*.

EXEUGENIA (Curran), 1953:4 (Volucella). Type-locality: Jamaica, St. Andrew, Constant Spring. Distr. - Jamaica. Type o AMNH*. N. COMB.

EXTERNum (Curran), 1939b:10 (Volucella). Type-locality: Peru, Río Marañón. Distr. - Peru. Type o AMNH*. N. COMB.

FLAVIPENNE (Wiedemann), 1830:203 (Volucella). Type-locality: "Brazil" Distr. - same. Type o ZMB. N. COMB.

FLAVISSIMUM (Giglio-Tos), 1892c:3 (Volucella). Type-locality: Mexico, Ori. zaba. Distr. - same. Type o UTOR. N. COMB.

FLAVIVENTRE Macquat, 1846:253 (125), pl. 10, figs. 16 (habitus), 16a (head). Type-locality: "Colombia, Venezuela". Distr. - same. Types o $+\mathrm{OXF}^{*}$ [types not marginatum Say, either caudatum Curran or species near it - JRVI. N. STATUS. 
Florella (Hull), 1944j:49 (Volucella). Type-locality: Guyana, Potaro River, Tumatumari. Distr. — same. Type \& CU. N. COMB.

FLUKeI (Curran, 1936b:10 (Volucella). Type-locality: Brazil, Paraná. Distr. - same. Type o $\mathrm{AMNH}^{*}$. N. COMB.

ForNAX (Townsend), 1895b:613 (Volucella). Type-locality: Mexico, Baja California, EI Taste. Distr. - U.S.A. (California, Arizona), Mexico. Type $\sigma^{7}$ CAS [destroyed]. N. COMB.

FRACTUM (Curran), 1926a:58 (Volucella). Type-locality: French Guiana, Cayenne. Distr. - same. Type o AMNH*. N. COMB.

FRAUDUlENTUM (Williston), 1891:48, pl. 1:13 (habitus), 13a (head), 13b (antenna) (Volucella). Type-locality: Mexico, Guerrero, Venta de Zopilote, $2800 \mathrm{ft}$; North Yucatán, Temax. Distr. - U.S.A. (California to Texas, south to Mexico (Guerrero, Yucatán). Types o \& $\mathrm{BM}(\mathrm{NH})+\mathrm{AMNH} *$. N. COMB.

FRAUENFELd (Schiner), 1868:358 (Temnocera). Type-locality: "Chile". Distr. - Chile, Argentina (Tucumán, La Rioja, Mendoza). Type o o V VMNH. Ref. - Shannon \& Aubertin, 1933:170, fig. 35a (head); Fluke, 1915b:19 (redescription), fig. 32 (scutellum). N. COMB.

Frontale (Sack), 1941:107 (1951:103) (Volucella). Type-locality: Peru, Tayapampa; Querobamba, $3520 \mathrm{~m}$.; Lake Titicaca. Distr. - Peru. Types of $q$ MD. N. COMB.

FUlvicoRne (Bigot), 1883b:84 (Volucella). Type-locality: "Panama". Distr. - same. Types ơ o $\mathrm{OXF}^{*}$. N. COMB.

FULNolucens (Walker), 1852:252 (Temnocera). Type-locality: "Brazil". Distr. - same. Type o $\mathrm{BM}(\mathrm{NH})$. N. COMB.

fulvolucus Williston, 1887a:28 (Temnocera), misspelling.

FUlvoNotatum (Bigot), 1875:476 (Volucella). Type-locality: Urugua: Montevideo. Distr. - same. Type o OXF*. N. COMB.

FuMipenne (Sack), 1941:104 (1951:108) (Volucella). Type-locality: Peru, Sivia; Peru; Costa Rica. Distr. - same. Types ơ o MD \& SMF. N. COMB.

Fumosum (Hull), 1943d:32 (Volucella). Type-locality: Brazil, Mt. Roraima, $8600 \mathrm{ft}$. Distr. - same. Type o $\mathrm{BM}(\mathrm{NH})$. N. COMB.

Furens (Giglio-Tos), 1892c:2 (Volucella). Type-locality: "Mexico". Distr. - same. Type or UTOR. N. COMB.

FUSCIPENNE (Macquart), 1842:84 (24), pl. 4, fig. 2 (habitus) (Volucella). Type-locality: "Brazil". Distr. - Brazil, Argenina (Tucumán). Type o MNHN. Ref. - Sack, 1921:136 (description), figs. 9 (larva), $11 \mathrm{~b}$ (spiracular horn). N. COMB.

Gertschi (Curran), 1939b:15 (Volucella). Type-locality: Panama, Chiriquí, El Volcan. Distr. - Guatemala, Panama. Type o AMNH*. N. COMB.

GIBBERUM (Schiner), 1868:358 (Temnocera). Type-locality: "Colombia". Distr. - same, Type of VMNH. Ref. - Curran, 1925b:246 (description o). N. COMB.

GoRGON (Hull), 1950c:233 (Volucella). Type-locality: Peru, Chanchamayo, 1000 m. Distr. - same. Type o Hull. N. COMB.

GRanulatum (Hull), 1944j:50 (Volucella). Type-locality: Peru, Río Pichis, Puerto Bermúdez. Distr. - same. Type o CU. N. COMB. GUIANICỦM (Hine), 1914:338 (Volucella). Type-locality: Guyana, Bartica. Distr. - same. Type of OhSU. N. COMB.

HaAgil (Jaennicke), 1867:397 (Volucella). Type-locality: "Mexico". Distr. - U.S.A. (California to Texas), south to Mexico (Oaxaca). 
Types o SMF? Ref, - Williston, 1887b:147 (description), pl. 5, fig. 8 (head). N. COMB.

hagii, Williston, 1887b:147, misspelling.

setigera Osten Sacken, 1877:334 (Temnocera). Type-locality: U.S.A., New Mexico, Vermejo River. Type $q$ MCZ.

HAMBletoNi (Fluke), 1951b:16, figs. 16 (head), 41 (antenna), 57 (male genitalia) (Volucella). Type-locality: Peru, Cañete. Distr. Ecuador, Peru. Type o $\mathrm{AMNH}^{*}$. N. COMB.

FIIRTIPES (Macquart), 1850:434 (130), pl. 12, figs. 13 (scutellum), 13a (hind leg) (Volucella). Type-locality: Bolivia, Yungas. Distr. Colombia, Brazil, Bolivia, Argentina (Misiones). Type of MNHN. N. COMB.

HORTICOLE (Hull), 1943d:21 (Volucella). Type-locality: Brazil, Amazon. Distr. - same. Type of BM(NH). N. COMB.

HoRvathi (Szilády), 1926:610, fig. 18 (habitus) (Graptomyza). Type-locality: "Cuba". Distr. - same. Type o DEI. N. COMB.

HYALINIPENNE (Hull), 1944f:36 (Volucella). Type-locality: "Brazil". Distr. - same. Type o Hull. N. COMB.

HYALOPTERUM (Giglio-Tos), 1892c:3 (Volucella). Type-locality: Mexico, Tampico. Distr. - same. Type of UTOR. N. COMB.

HYDROFENESTRA (Hull), 1943n:513 (Volucella). Type-locality: Colombia, Muzo. Distr. - same. Type o Hull. N. COMB.

HystRIX (Giglio-Tos), 1892c:4 (Volucella). Type-locality: Mexico, Tuxpango. Distr. - same. Type o UTOR. N. COMB.

Imitans (Curran), 1926a:63 (Volucella). Type-locality: Mexico. Distr. same. Type o AMNH*. N. COMB.

IMPRESSUM (Hull), 1949f:30 (Volucella). Type-locality: Brazil, Santa Catarina, Nova Teutônia. Distr. - same. Type ơ Hull. N. COMB.

Incommodum (Knab), 1916a:93 (Volucella). Type-locality: Panama, Canal Zone, Ancón. Distr. - same. Types ơ $q$ USNM. N. COMB.

InCONSIstens (Curran), 1939b:3 (Volucella). Type-locality: Brazil, Mt. Roraima, $5400 \mathrm{ft}$. Distr. - Costa Rica, Brazil. Type \&. AMNH*. N. COMB.

INQUISITOR (Hull), 1943d:26 (Volucella). Type-locality: Central America. Distr. - Mexico, Central America. Type o* BM(NH). N. COMB. INTEGRUM (Walker), 1857:155 (Temnocera). Type-locality: "Valley of Amazon". Distr. - same. Type ? BM(NH). N. COMB.

INToNA (Curran), 1928b:22 (Volucella). Type-locality: Jamaica, Gordon Town. Distr. - same. Type o BM(NH). N. COMB.

ISABELLINA (Williston), 1887b:140 (Volucella). Type-locality: U.S.A., Arizona. Distr. - U.S.A. (California to Colorado), south to Mexico (Sonora). Type o USNM. N. COMB.

JoHnsoni (Curran), 1925b:249 (Phalacromyia). Type-locality: Venezuela, Caura Valley. Distr. - same. Type o MCZ. N. COMB.

KAHLI (Hull), 1938:122, fig. 5 (abdomen) (Volucella). Type-locality: French Guiana, Mana River. Distr. - same. Type o CM. N. COMB.

LACTICOERUleuM (Hull), 1944f:35 (Volucella). Type-locality: Brazil, São Paulo, Praia Grande do Rio Feio, Penápolis. Distr. - same. Type o Hull. N. COMB.

LANEI (Curran), 1936b:11 (Volucella). Type-locality: Brazil, São Paulo, Juquiá. Distr. - Brazil (São Paulo, Santa Catarina). Type o AMNH*. N. COMB.

Latevittatum (Curran), 1939b:16 (Volucella). Type-locality: Colombia, between Queremal and Buenaventura. Distr. - Colombia, Peru, Bolivia. Type ơ AMNH*. N. COMB. 
LATUM (Wiedemann), 1830:195 (Volucella). Type-locality: Mexico, Oaxaca. Distr. - Mexico. Type क ZMB. N. COMB.

lacta, Bigot, 1883b:76, misspelling.

LIMBIPENNE Williston, 1887b:152. Type-locality: Mexico, Isthmus of Tehuantepec. Distr. - U.S.A. (Texas), Mexico. Type $q$ USNM. Ref. - Williston, 1891:pl. 2:2 (habitus), 2a (head), 2b (antenna). N. COMB.

lucasana Townsend, 1895b:615 (Volucella). Type-locality: Mexico, Baja California, El Taste. Type + CAS [destroyed].

lucana, Townsend, 1895a:44, misspelling.

LiRIOPE (Hull), 1949f:28 (Volucella). Type-locality: Brazil, Santa Catarina, Nova Teutônia. Distr. - same. Type o Hull. N. COMB.

LuCiliA (Hull), 1950a:65 (Volucella). Type-locality: Peru, Chanchamayo. Distr. - same. Type o Hull. N. COMB.

LUGENS (Wiedemann), 1830:206 (Volucella). Type-locality: "Brazil". Distr. - Guatemala, Brazil. Type \& VMNH. Ref. - Curran, 1926a:65 (redescription). N. COMB.

LUMina (Hull), 1937a:168, fig. 8 (abdomen) (Volucella). Type-locality: Bolivia, Ramupasa. Distr. - same. Type of USNM. N. COMB.

LUNUlfFerum (Hull), 1937a:167 (Volucella). Type-locality: Peru, Iquitos. Distr. - same. Type o USNM. N. COMB.

MACQUARTI (Curran), 1926a:60 (Volucella). Type-locality: Honduras, Prieta. Distr. - Mexico, Honduras, Trinidad, Guyana, Brazil, Peru, Bolivia. Type of AMNH*. N. COMB.

MACrocephalum (Giglio-Tos), 1892e:45 (Camerania). Type-locality: "Mexico". Distr. - U.S.A. (Arizona), Mexico. Type o UTOR. N. COMB.

MACRORHINUM (Bigot), 1875:474 (Volucella). Type-locailty: "Brazil". Distr. - same. Type ot OXF. N. COMB.

MACULA (Wiedemann), 1830:200 (Volucella). Type-locality: "Brazil". Distr. - Mexico (Tabasco), Guyana, Brazil (Mato Grosso). Types o 아 VMNH. N. COMB.

MACUloIdes (Curran), 1939b:10 (Volucella). Type-locality: Brazil, Mato Grosso, Chapada. Distr. - same. Type o AMNH. N. COMB.

MARCELI (Curran), 1939b:7 (Volucella). Type-locality: Brazil, São Paulo. Distr. - same. Type o* AMNH. N. COMB.

Marginatum (Say), 1830:167 (Volucella). Type-locality: Mexico. Distr. U.S.A. (California to Colorado), south to Mexico. Type ? Lost. Ref. - Williston, 1887b:151 (description), pl. 7:1 (wing), 1a (head), 1b (antenna); Curran, 1925a:206, pl. 10:146 (male genitalia).

distinctum Giglio-Tos, 1892e:41, fig. 15 (abdomen). Type-locality: Mexico, Tehuacán. Types ơ o UTOR.

fax Townsend, 1895a:42 (Volucella). Type-locality: U.S.A., Colorado, Fort Collins. Type $0^{*}$ ?.

inops Townsend, 1895a:43 (Volucella). Type-locality: U.S.A., Colorado, Fort Collins. Type of UK.

toltec Townsend, 1895a:45 (Volucella). Type-locality: Mexico, Guanajuato. Type $\sigma^{*}$ ?

tolteca, Townsend, 1895d:6, misspelling.

estebana Townsend, 1895b:612 (Volucella). Type-locality: Mexico, Baja California, San Esteban. Types ơ $\sigma^{*}$ CAS [destroyed]).

sodomis Townsend, 1895b:616 (Volucella). Type-locality: Mexico, Baja California, El Taste. Types o CAS [destroyed].

MEgaCePhalum (Loew), 1863:305 (1864b:189) (Temnocera). Type-locality: U.S.A., California. Distr. - U.S.A. (California), Mexico. Type + 
MCZ. Ref. - Williston, 1887b:146 (description). N. COMB.

MELLEUM (Jaennicke), 1867:396 (Volucella). Type-locality: "Mexico". Distr. - Mexico (Guanajuato, Distrito Federal). Types on o SMF. N. COMB.

MERETRICIAS (Williston), 1888:272 (Volucella). Type-locality: Brazil, Mato Grosso, Chapada; Rio de Janeiro. Distr. - Brazil (Rio de Janeiro, Santa Catarina, Mato Grosso), Argentina (Misiones). Types of o AMNH*. N. COMB.

METALLIFERUM (Walker), p.p., 1849:636 (Volucella). Type-locality: Mexico [specimen from Venezuela under dispar Macquart(q.v.)]. Distr. - U.S.A. (California to Texas), south to Mexico (Yucatán). Type +. BM(NH). Ref. - Curran, 1925a:206, pl. 10:143 (male genitalia); Curran, 1953:7 (revision). N. COMB.

violaceum Say, 1830:166 (Volucella; preocc. Lepeletier \& Serville, 1825). Type-locality: "Mexico". Type(s) o lost.

maximitiani Jaennicke, 1867:395 (Volucella). Type-locality: "Mexico". Types of o SMF.

Metallorum (Walker), 1852:252 (Temnocera). Type-locality: Brazil, Pará. Distr. - same. Type o BM(NH). N. COMB.

MexicANum (Macquart), 1842:85 (25), pl. 5, figs. 3 (habitus), 3a (head) (Volucella). Type-locality: "Mexico". Distr. - U.S.A. (California to Georgia, south to Mexico (Yucatán). Type of MNHN*. Ref. - Williston, 1887b:137 (redescription), pl. 6, figs. 5 (wing), 5a (head). N. COMB.

nigrum Greene, 1923:165 (Volucella). Type-locality: U.S.A., florida, Miami. Type ơ USNM*. N. SYN.

minimum (Giglio-Tos), 1892c:3 (Volucella). Type-locality: Mexico, Orizaba. Distr. - same. Type o UTOR. N. COMB.

Missionera (Lynch Arribálzaga), 1892b:183 (1892c:134) (Volucella). Typelocality: Uruguay, Misiones. Distr. - same. Type \& MACN. N. COMB.

MOCANUM (Curran), 1936b:11 (Volucella). Type-locality: Guatemala, Moca Guatalón. Distr. - Guatemala, Peru, Venezuela. Type o $\mathrm{AMNH}^{*}$. N. COMB.

MORPHo (Curran), 1939b:2 (Volucella). Type-locality: Brazil, Pernambuco. Distr. - same. Type o AMNH*. N. COMB.

MUS (Williston), 1888:274 (Volucella). Type-locality: Brazil, Mato Grosso, Chapada. Distr. - Brazil (Mato Grosso), Peru, Argentina (Tu-

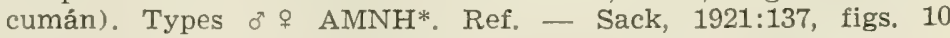
(puparium), 11c (spiracular horn). N. COMB.

MUSANUm (Curran), 1930d:16 (Volucella). Type-locality: Panama, Canal Zone, Barro Colorado Island. Distr. - same. Type of ÁMNH*. N. COMB.

MUSCARIUM (Thomson), 1869:493 (Haurotricha). Type-locality: Argentina, Buenos Aires. Distr. - Colombia, Argentina. Type o NRS*. N. COMB.

argentina Bigot, 1883b:83 (Phalachromyia). Type-locality: Argentina, Buenos Aires. Type or BM(NH).

MUSICANUm (Curran), 1930d:15 (Volucella). Type-locality: Brazil, Mato Grosso, Chapada. Distr. - same. Type o AMNH*. N. COMB.

MUstoides (Curran), 1927c:84 (Volucella). Type-locality: Honduras, Prieta. Distr. - same. Type o AMNH*. N. COMB.

NASICUM (Williston), 1891:55, pl. 2:1 (habitus), la (head), 1b (antenna) (Ophromyia). Type-locality: Mexico, Guerrero, Acaguizotla. Distr. - Mexico. Types o BM(NH). Ref. - Hull, 1949d:349, fig. 20e, $20 \mathrm{~g}$ (head). N. COMB. 
NAUtLANum (Townsend), 1897b:28 (Volucella). Type-locality: Mexico, Vera Cruz, Río Nautla, San Rafael. Distr. - same. Types o $\mathrm{BM}(\mathrm{NH})$. N. COMB.

NEOSPLENDENS Thompson (nom nov. for splendens Hull). Distr. - Brazil (Santa Catarina). NOM. NOV.

splendens Hull, 1949f:26 (Volucella; preocc. Townsend, 1897). Typelocality: Brazil, Santa Catarina, Nova Teutônia. Type o Hull. Ref. - Fluke, 1951b:27 (descriptive note).

NEOTROPICUM Thompson (nom. nov. for vesiculosum Fabricius, 1805). Distr. - South America. NOM. NOV.

vesiculosum Fabricius, 1805:226 (Syrphus; preocc. Fabricius, 1794). Type-locality: "America meridionali". Type ? MC.

NIGRIFACIES (Bigot), 1875:479 (Volucella). Type-locality: "Mexico". Distr. same. Type $0^{\pi} \mathrm{BM}(\mathrm{NH})$. N. COMB.

NIGRIFRoNS (Hine), 1914:340 (Phalacromyia). Type-locality: Guyana, Bartica. Distr. - same. Type o OhSU. N. COMB.

NIGRIPES (Bigot), 1857a:296, pl. 7:9 (head) (Phalacromyia). Type-locality: "Chile". Distr. - Peru, Chile. Type o OXF*. N. COMB.

concolor Philippi, 1865:735 (Phalacromyia). Type-locality: Chile, Prov. Santiago. Type(s) ? MNHNS. N. SYN.

Nigropodum (Hull), 1949f:35 (Volucella). Type-locality: Peru, Pucallpa. Distr. - same. Type or Hull. N. COMB.

Nigroviride (Hull), 1949h:37 (Volucella). Type-locality: Peru, Pucallpa. Distr. - same. Type of Hull. N. COMB.

NITIDIGASTER (Hull), 1937a:165 (Volucella). Type-locality: Argentina, Tucumán, Villa Nogués. Distr. - same. Type o USNM. N. COMB.

Notatum (Bigot), 1875:475 (Volucella). Type-locality: Uruguay, Montevideo. Distr. - same. Types of o OXF*. N. COMB.

OBLIQUICORNE (Curran), 1939b:12 (Volucella). Type-locality: Guyana, Tumatumari. Distr. - same. Type o AMNH*. N. COMB.

OBSCURIPENNE (Lynch Arribálzaga), 1892b:180 (1892c:130 (Volucella). Type-locality: Argentina, Misiones, Monteagudo. Type ? MACN. N. COMB.

oBSCURIUS (Curran), 1939b:5 (Volucella). Type-locality: Brazil, Santa Catarina, Nova Teutônia. Distr. - Brazil (Santa Catarina), Argentina (Tucumán, La Rioja). Type ơ AMNH. N. COMB.

oestroides (Hull), 1943d:23 (Volucella). Type-locality: Brazil, Rio de Janeiro. Distr. - same. Type o $\mathrm{BM}(\mathrm{NH})$. N. COMB.

omochroma (Giglio-Tos), 1892c:2 (Volucella). Type-locality: "Mexico". Distr. - same. Type of UTOR. N. COMB.

opAlicolor (Hull), 1943d:36 (Volucella). Type-locality: Bolivia, Prov. Sara, Santa Cruz de la Sierra. Distr. - same. Type of BM(NH). N. COMB.

opeostoma (Hull), 1949h:32 (Volucella). Type-locality: Panama, Chiriquí, Volcán, Bambito. Distr. - same. Type \& Hull. N. COMB.

OPINATOR (Williston), 1891:51, pl. 1:14 (habitus), 14a (head), 14b (antenna) (Volucella). Type-locality: Mexico, Vera Cruz, Atoyac. Distr. - same. Type o BM(NH). N. COMB.

ORNATUM (Williston), 1891:49 (Volucella). Type-locality: Mexico, Jalapa. Distr. - same. Types o BM(NH) + AMNH*. N. COMB.

hispida Giglio-Tos, 1892c:4 (Volucella). Type-locality: Mexico, Orizaba. Type ơ UTOR.

oSBURNI (Hull), 1942a:92 (Volucella). Type-locality: Paraguay, Villarica. Distr. - Paraguay, Argentina (Tucumán). Type ${ }^{*} \mathbf{M C Z}^{*}$. N. COMB. 
PACHecor (Curran), 1939b:13 (Volucella). Type-locality: Guatemala. Distr. - same. Type \& AMNH*. N. COMB.

PALlenS (Wiedemann), 1830:204 (Volucella). Type-locality: "Brazil". Distr. - U.S.A. (Arizona to North Carolina), south to Argentina and Brazil. Types o VMNH + SMF*. Ref. - Williston, 1887b:147 (redescription), pl. 6:2 (wing); Sack, 1921:137, fig. 11a (spiracular horn). N. COMB.

sexmaculatum Palisot de Beauvois, 1819:224, pl. 3:8 (habitus) (Syrphus). Type-locality: Dominican Republic, Santo Domingo, Southern U.S.A. Types ? ? N. SYN. [nomen oblitum].

testaceum Rondani, 1848:67 (Volucella). Type-locality: Brazil. Type o MIZUN.

sexpunctatum Loew, 1861:38 (Volucella). Type-locality: "Cuba". Type o MCZ.

var. QUADRIPUNCTATUM (Doesburg), 1963:17, 30. Type-locality: Surinam, Paramaribo. Distr. - same. Type $\sigma^{*}$ Doesburg.

Pallidithorax (Hull), 1941g:156, fig. 8 (mesonotum) (Volucella). Typelocality: Honduras, Rosario Mires. Distr. - same. Type + MCZ*. N. COMB.

PALlidum (Macquart), 1842:86 (26) (Volucella). Type-locality: "Guyana". Distr. - same. Type $\sigma^{*} \mathrm{MNHN}^{*}$. N. COMB.

PALlisteri (Curran), 1953:9 (Volucella). Type-locality: Mexico, Distrito Federal, Tlalpán. Distr. - same. Type o AMNH*. N. COMB.

PAlmyra (Hull), 1949f:33 (Volucella). Type-locality: Brazil, Santa Catarina, Nova Teutônia. Distr. - same. Type ơ Hull. N. COMB.

PANAMENSE (Curran), 1930d:17 (Volucella). Type-locality: Panama, Canal Zone, Barro Colorado Island. Distr. - Panama. Type o AMNH*. N. COMB.

PANAMENum (Curran), 1939b:12 (Volucella). Type-locality: Panama, Canal Zone, Barro Colorado Island. Distr. - Panama, Peru. Type o AMNH*. N. COMB.

PARANA (Hull), 1942h:23 (Volucella). Type-locality: Brazil, Paraná. Distr. - same. Type o Hull. N. COMB.

PARINA (Fluke), 1951b:25, figs. 14 (head), 61 (male genitalia) (Volucella). Type-locality: Chile, Arica, Parinacota. Distr. - same. Type o UCSC. N. COMB.

PARvUM (Rondani), 1848:66 (Volucella). Type-locality: "Brazil". Distr. same. Type o MIZUN. N. COMB.

PECToRale (Rondani), 1863:3 (Volucella). Type-locality: "America meridion.". Distr. - same. Types o $q$ MIZUN. N. COMB.

PERlatum (Hine), 1914:339 (Volucella). Type-locality: Guatemala, Los Amates. Distr. - same. Type or OhSU. N. COMB.

PERSimile (Williston), 1888:273 (Volucella). Type-locality: Brazil, Destares (?); Mato Grosso, Chapada; Rio de Janeiro. Distr. - same. Types ot $\& \mathrm{AMNH}^{*}$. N. COMB.

PERTINAX (Hull), 1950a:67 (Volucella). Type-locality: Peru, Chanchamayo. Distr. - same. Type o Hull.

PERUVIANUM (Vimmer \& Soukup), 1938:28 (Volucella). Type-locality: Peru, Puno. Distr. - same. Type o NMPP*. N. COMB.

PICA (Schiner), 1868:355 (Phalocromyia). Type-locality: Colombia. Distr. - Mexico (Veracruz), Colombia, Venezuela. Types of $\mathrm{VMNH}$ N. COMB.

pictum Schiner, 1868:pl. 4:6a + b (habitus) (Phalacromyia; preocc. Wiedemann, 1830), incorrect original spelling of pica.

PICtoides (Hull), 1941e:280 (Volucella). Type-locality: Cuba, Havana. Distr. - same. Type \& Hull. N. COMB. 
PICtUM (Wiedemann), 1830:201 (Volucella). Type-locality: "Brazil". Distr. - Mexico (Guerrero, Veracruz, Yucatán), Cuba, Panama, Brazil (Mato Grosso, Santa Catarina), Argentina (Tucumán). Types of क VMNH. Ref. - Curran, 1925a:206, pl. 10:145 (male genitalia); Doesburg, 1966:96, fig. 66 (habitus). N. COMB.

PICTURATUM (Lynch Arribálzaga), 1892b:184 (1892c:135) (Volucella). Typelocality: Argentina, Misiones, Picada de Paggi. Distr. - same. Types \& MACN. N. COMB.

PINKUSI (Curran), 1938:1 (Volucella). Type-locality: Trinidad, St. Anne. Distr. - same. Type o $\mathrm{AMNH}^{*}$. N. COMB.

Placivum (Hull), 1943d:30 (Volucella). Type-locality: Ecuador, Santa Inez. Distr. - same. Type o BM(NH). N. COMB.

Plaumanni (Curran), 1939b:4 (Volucella). Type-locality: Brazil, Santa Catarina, Nova Teutônia. Distr. - Brazil (Santa Catarina), Argentina (Salta). Type o AMNS*. N. COMB.

Plorans (Rondani), 1848:65 (Volucella). Type-locality: "Brazil". Distr. same. Type or MIZUN. N. COMB.

polorans, Williston, 1886:317, misspelling.

Posticum (Say), 1830:166 (Volucella). Type-locality: "Mexico". Distr. U.S.A. (California to Colorado), south to Mexico. Type $0^{\pi}$ lost. N. COMB.

castanea Bigot, 1875:476 (Volucella). Type-locality: Mexico, Oaxaca. Type $0^{*} \mathrm{OXF}^{*}$.

var. PALlidius (Giglio-Tos), 1892c:50 (Volucella). Type-locality: Mexico, Tehuacan. Type \& UTOR.

PRASINUS (Schiner), 1868:354 (Phalacromyia). Type-locality: "South America". Distr. - same. Types o o VMNH. N. COMB.

PRESCUTELlaRE (Williston), 1888:273 (Volucella). Type-locality: Brazil, Mato Grosso, Chapada. Distr. - Brazil (Mato Grosso), Argentina (Misiones). Types of of AMNH*. N. COMB.

PRoCTERI (Curran), 1939b:9 (Volucella). Type-locality: Brazil, Santa Catarina, Nova Teutônia. Distr. - same. Type ơ AMNH*. N. COMB.

PUBESCENS (Loew), 1861:38 (Temnocera). Type-locality: "Cuba". Distr. same. Type o MCZ? Ref. - Williston, 1887b:148 (redescription). N. COMB.

PUlchrapuella (Hull), 1943d:33 (Volucella). Type-locality: Jamaica, Kingston. Distr. - same. Type $\sigma^{7} \cdot \mathrm{BM}(\mathrm{NH})$. N. COMB.

PUlchripes (Bigot), 1875:480 (Volucella). Type-locality: "Mexico". Distr. - same. Type $\sigma^{*} \mathrm{OXF}^{*}$. N. COMB.

PULCHRUM (Williston), 1891:41 (Phalacromyia). Type-locality: Costa Rica, Río Sucio. Distr. - same. Type of o BM(NH). N. COMB.

PunCTIFERUM (Bigot), 1875:475 (Volucella). Type-locality: "Amazonia". Distr. - Colombia. Type o $\mathrm{BM}(\mathrm{NH})$. N. COMB.

PUNCTIGENA (Hull), 1937a:165, fig. 6 (abdomen) (Volucella). Type-locality: Guatemala, Siquinola. Distr. - same. Type q USNM. N. COMB.

PURPURASCENS (Loew), 1869:31 (1872b:145) (Temnocera). Type-locality: "Haiti". Distr. - same. Type $\sigma^{*} \mathrm{MCZ}$ (lost). Ref. - Williston, 1887b:148 (redescription). N. COMB.

watsoni Curran, 1930f:3 (Volucella). Type-locality: Haiti, Port-auPrince. Type ơ AMNH*. N. SYN.

PURPUREUM (Walker), 1849:637 (Volucella). Type-locality: Brazil, Pará. Distr. - same. Type ơ BM(NH). Ref. - Sack, 1921:137 (description), fig. 11d (spiracular horn), 15 (larva). N. COMB.

PURPURIFERUM (Bigot), 1875:477 (Volucella). Type-locality: Mexico, Oaxaca. Distr. - same. Type o OXF*. N. COMB. 
PUSIllum (Macquart), 1842:81 (21), pl. 5, figs. 1 (habitus), 1a (head) (Volucella). Type-locality: "Cuba". Distr. - U.S.A. (Texas to Florida); Cuba. Type $\uparrow$ [Lille?]. Ref. - Williston, 1887b:144 (redescription), pl. 6:3 (wing), 3a (head), N. COMB.

QUADRATUM (Williston), 1891:46, pl. 1:12 (habitus), 12a (head), 12b (antenna) (Volucella). Type-locality: Mexico, Guerrero, Chilpancingo. Distr. - U.S.A. (Arizona), Mexico (Guerrero). Type \& BM(NH). N. COMB.

RaFaelanum (Townsend), 1897b:28 (Volucella). Type-locality: Mexico, Veracruz, Río Nautla, San Rafael. Distr. - same. Type o BM (NH), N. COMB.

RECTUM (Wulp), 1882:125 (Temnocera). Type-locality: "Argentina". Distr. - Argentina (Tucumán, Salta). Types o $q$ lost?. Ref. Fluke, 1951b:7 (description), figs. 1 (head), 49 (male genitalia). N. COMB.

RHEA (Hull), 1950c:232 (Volucella). Type-locality: Peru, Matucana. Distr. - same. Type o* Hull. N. COMB.

RoBUSTUM (Sack), 1941:109 (1951:105) (Temnocera). Type-locality: Peru, Ocana, $2600 \mathrm{~m}$. Distr. - same. Types of o HZM. N. COMB.

Roraima (Curran), 1939b:3 (Volucella). Type-locality: Brazil, Mt. Roraima, $6900 \mathrm{ft}$. Distr. - same. Type ot AMNH*. N. COMB.

RCSA (Curran), 1939b:7 (Volucella). Type-locality: Peru, Middle Río Ucayali. Distr. - same. Type or AMNH*. N. COMB.

ROSPIGLIOSII (Brèthes), 1920:31 [also 1937:ii] (Volucella). Type-locality: Peru, Chanchamayo region. Type ? MACN. N. COMB.

RUFITARSE Thompson (nom. nov. for tibiale Shannon). Distr. - Brazil (Santa Catarina), Argentina (Misiones). NOM. NOV.

tibiale Shannon, 1929:575 (Volucella; preocc. Macquart, 1846). Typelocality: Argentina, Misiones. Type o MACN.

RUfoscutellare (Philippi), 1865:735 (Phalacromyia). Type-locality: Chile, prov. Santiago. Distr. - Chile (Aconcagua, Santiago). Type ? MNHNS. N. COMB.

RURAle (Curran), 1939b:2 (Volucella). Type-locality: Brazil, Santa Catarina, Nova Teutônia. Distr. - same. Type ơ AMNH*. N. COMB.

SALTI Dobroscky, in Curran, 1930d:3. Type-locality: Colombia, Santa Maria. Distr. - same. Type o AMNH*.

SAPHIRINUM (Bigot), 1883b:85 (Volucella). Type-locality: "Chile". Distr. Chile (Valparaíso - Curicó). Types of of $\mathrm{BM}(\mathrm{NH})$. N. COMB.

sapphirina, Shannon \& Aubertin, 1933:168, emend.

SAPPHO (Hull), 1949h:34 (Volucella). Type-locality: Panama, Canal Zone. Summit. Distr. - Panama. Type " $\sigma^{*}$ Hull. N. COMB.

Schwarzi Curran, 1935:4. Type-locality: "Venezuela, Curaçao". Distr. same. Type o AMNH*. N. COMB.

Scintilans (Hull), 1949f:39 (Volucella). Type-locality: Peru, Pucallpa. Distr. - same. Type o Hull. N. COMB.

SCUTELlatum (Macquart), 1842:85 (25), pl. 6, figs. 2 (habitus), 2a (head), 2b (scutellum) (Volucella). Type-locality: "Chile". Distr. - Brazil (Mato Grosso), Peru (Lima), Chile (Tarapacá — Santiago). Type o MNHN*. Ref. - Fluke, 1951b:4 (redescription), figs. 18 (wing tip), 21 (scutellum), 36 (antenna), 47 (male genitalia). N. COMB.

andicolum Bigot, 1884a:548 (Temnocera). Type-locality: "Chile". Type o $\mathrm{BM}(\mathrm{NH})$.

SELECTUM (Curran), 1939b:15 (Volucella). Type-locality: Brazil, Santa Catarina, Nova Teutônia. Distr. - same. Type or AMNH*. N. COMB. 
SEPIA (Hull), 1943d:25 (Volucella). Type-locality: "Central America". Distr. - same. Type o $\mathrm{BM}(\mathrm{NH})$. N. COMB.

SHANNONI Thompson (nom. nov. for salti Curran). Distr. - Colombia. NOM. NOV.

salti Curran, 1930d:12 (Volucella; preocc. Dobroscky, 1930). Typelocality: Colombia, Río Frío, Magdalena. Type ơ AMNH*.

SICA (Curran), 1953:9 (Volucella). Type-locality: Ecuador, Tungurahua, Baños. Distr. - same. Type o AMNH*. N. COMB.

Simile (Giglio-Tos), 1892c:2. Type-locality: Mexico, Tehuacán \& Meztillán. Distr. - U.S.A. (Arizona, New Mexico), Mexico. Types $\delta$ ? UTOR*.

parvum Giglio-Tos, 1892c2 (preocc. Rondani, 1848). Type-locality: Mexico, Tehuacán. Types $\sigma^{*}$ o UTOR [not found - JRV].

SMITHAE (Thompson), 1965:61 (Lepidosis). Type-locality: Argentina, La Rioja, Mascasin. Distr. - Argentina (La Rioja). Type ơ Thompson*. N. COMB.

SOROR (Bigot), 1883b:83 (Phalacromyia). Type-locality: Argentina, Buenos Aires. Distr. - same. Type of $(\mathrm{BM}(\mathrm{NH})$. N. COMB.

SOUKUPII (Vimmer \& Soukup), 1938:29 (Volucella). Type-locality: Peru, Puno. Distr. - same. Type o NMPP*. N. COMB.

SPECtrale (Hull), 1942h:21 (Volucella). Type-locality: "Brazil". Distr. same. Type i Hull. N. COMB.

SPINIGERUM (Wiedemann), 1830:197 (Volucella). Type-locality: Uruguay, Montevideo. Distr. - Uruguay, Argentina (Tucumán, Buenos Aires, Santa Fé, Salta, Chaco, Formosa, Córdoba). Types o $q$ VMNH \& AMNH*. Ref. - Shannon \& Aubertin, 1933:170, fig. 35b (head); Fluke, 1951b:18 (redescription), figs. 11 (head), 20 (wing tip), 31 (scutellum), 42 (antenna), 58 (male genitalia). N. COMB.

fulvicorne Bigot, 1884a:547 (Temnocera?; preocc. Bigot, 1883). Type-locality: Argentina, Buenos Aires. Types $\sigma^{\circ}+\mathrm{B} \mathrm{BM}(\mathrm{NH})$.

SPINITHORAX (Lynch Arribálzaga), 1892b:190 (1892c:141) (Temnocera). Type-locality: Argentina, Chaco, Colonia Azara. Distr. - Brazil (São Paulo, Santa Catarina, Paraná), Paraguay, Argentina. Type \& MACN. Ref. - Fluke, 1951b:20 (redescription), figs. 33 (scutellum), 59 (male genitalia). N. COMB.

var. Novum (Fluke), 1951b:22 (Volucella). Type-locality: Brazil, Santa Catarina, Nova Teutônia. Type \& AMNH.

SPLENDENS (Townsend), 1897b:29 (Volucella; as var. of opalina). Typelocality: Mexico, Veracruz, Río Nautla, San Rafael. Distr. same. Types of $\mathrm{BM}(\mathrm{NH})$. N. COMB.

var. TOWNSENDI (Goot), 1964:214 (nom. nov. for opalina Townsend). Distr. - Mexico (Vera Cruz).

opalina Townsend, 1897b:29 (Volucella; preocc. Wiedemann, 1830). Type-locality: Mexico, Veracruz, Río Nautla, San Rafael. Types of $f \mathrm{BM}(\mathrm{NH})$.

SQUAMIGERUM (Curran), 1925c:348 (Volucella). Type-locality: Peru, Río Pichis, Puerto Bermúdez. Distr. - same. Type o CU. N. COMB. STERNALE (Curran), 1930d:20 (Volucella). Type-locality: Mexico, Baja California Sur, San José del Cabo. Distr. - U.S.A. (Arizona), Mexico (Baja California Sur). Type o AMNH*. N. COMB.

Stigmatum (Hull), 1949f:37 (Volucella). Type-locality: Peru, Pucallpa. Distr. - same. Type o Hull. N. COMB.

Subcoeruleum (Rondani), 1863:3 (Phalacromyia). Type-locality: "America meridionalis". Distr. - same. Type o MIZUN. N. COMB.

Sublatum (Fluke), 1950b:451, figs. 17 (head), 18 (male genitalia) (Volu- 
cella). Type-locality: Chile, Arica, Putre, 3650 m. Distr. - Argentina (Jujuy), Chile (Tarapacá). Type o UCSC. N. COMB.

Submetallicum (Rondani), 1848;67 (Phalacromya). Type-locality: "Brazil". Distr. - same. Type o MIZUN. N. COMB.

SUbrostratum (Rondani), 1848:68 (Phalacromya). Type-locality: "Brazil". Distr. - Brazil, Mexico? Type or MIZUN. N. COMB.

?triste Bigot, 1875:482 (Volucella). Type-locality: "Mexico". Type o OXF*.

?melanorhina Bigot, 1883b:86 (Phalacromyia). Type-locality: "Mexico". Type \& BM(NH).

SUltzi (Curran), 1939b:7 (Volucella). Type-locality: Brazil, São Paulo, Alto da Serra (=Paranapiacaba). Distr. - same. Type o AMNH*. N. COMB.

TAMAUlipanum (Townsend), 1898b:51 (Volucella). Type-locality: U.S.A., Texas, near Brownsville. Distr. - U.S.A. (Texas, Louisana), south to Mexico (Yucatán); Hawaii [immigrant]. Types $\sigma^{\prime} \uparrow$ $\mathrm{BM}(\mathrm{NH})$. N. COMB.

timberlakei Curran, 1926a:63 (Volucella). Type-locality: U.S.A., Texas. Type o $\mathrm{CNC}^{*}$.

femina Curran, 1930f:4 (Volucella). Type-locality: Mexico, Yucatán, Chichén Itzá. Type † AMNH*.

TATEI (Curran), 1930d:21 (Volucella). Type-locality: Venezuela, Mt. Duida. Distr. - same. Type o $\mathrm{AMNH}^{*}$. N. COMB.

TAU (Bigot), 1883b:84 (Volucella). Type-locality: "Mexico". Distr. U.S.A. (Oregon to Colorado), south to Mexico. Type of BM(NH). Ref. - Williston, 1887b:143 (redescription), pl. 6:13 (antenna). N. COMB.

TEFFERA (Curran), 1939b:9 (Volucella). Type-locality: Brazil, Amazonas, Tefé. Distr. - same. Type of AMNH*. N. COMB.

TIBIALE (Macquart), 1846:251 (123) (Volucella). Type-locality: Mexico, Yucatán, Merida. Distr. - same. Type o MNHN? [not found JRV]. N. COMB.

TRICINCTUM (Bigot), 1875:477 (Volucella). Type-locality: Mexico, Oaxaca. Distr. - U.S.A. (Texas), Mexico, Honduras, Guatemala, Costa Rica, Brazil, Argentina (Tucumán, Salta, Misiones); Hawaii [immigrant]. Type o OXF*. N. COMB.

hoya Curran, 1947b:1 (Volucella). Type-locality: Hawaii, Oahu, Honolulu. Type of AMNH*.

TRIFASCIUM (Walker), 1857:154 (Temnocera). Type-locality: "Valley of Amazon". Distr. - same. Type ? BM(NH). N. COMB.

TRIGoNum (Giglio-Tos), 1892c:3 (Volucella). Type-locality: Mexico, Orizaba. Distr. - same. Types ơ UTOR. N. COMB.

TRIPUNCTATUM (Hull), 1949f:31 (Volucella). Type-locality: Brazil, Santa Catarina, Nova Teutônia. Distr. - same. Type ơ Hull. N. COMB. TRITUBERCULATUM Thompson (nom. nov. for scutellatum Williston). Distr. - Brazil, Argentina (Misiones). NOM. NOV.

scutellatum Williston, 1888:277 (Apophysophora; preocc. Macquart, 1842). Type-locality: Brazil, Mato Grosso, Chapada. Types of $q$ AMNH*.

TUMicephalum (Hull), 1943d:35 (Volucella). Type-locality: "Peru". Distr. - same. Type o BM(NH). N. COMB.

TYMPANITIS (Fabricius), 1805:226 (Syrphus), Type-locality: "America meridionali". Distr. - Mexico, Guatemala, Costa Rica, Panama, Venezuela, Peru, Brazil. Types ơ o MC*. N. COMB.

ardua Wiedemann, 1830:204 (Volucella). Type-locality: Surinam. Types o ZMB \& VMNH. 
UlRICA (Hull), 1950a:68 (Volucella). Type-locality: Peru, Chanchamayo. Distr. - same. Type \& Hull. N. COMB.

UNICOLOR (Curran), 1925c:347 (Volucella). Type-locality: Venezuela, C. - Bolivar. Distr. - same. Type o MCZ. N. COMB.

UNILECTUM (Walker), 1860:292 (Temnocera). Type-locality: "Mexico". Distr. — same. Type o BM(NH). N. COMB.

UNIPUNTATUM (Curran), 1926a:63 (Volucella). Type-locality: West Indies, Desecheot Island. Distr. - U.S.A. (Texas? [Wirth et al., 1965: 602]); Puerto Rico. Type o AMNH*. N. COMB.

VACUM (Fabricius), 1775:763 (Syrphus). Type-locality: "America". Distr. - Jamaica. Type ? ?. Ref. - Townsend, 1895a:46 (redescription). N. COMB.

VAgoides (Curran), 1927c:86 (Volucella). Type-locality: Costa Rica, San José. Distr. - Guatemala, Costa Rica. Type + UK [Byers, et al., 1962]. N. COMB.

VAGUM (Wiedemann), 1830:205 (Volucella). Type-locality: "Brazil". Distr. - Mexico, Guyana, Peru, Brazil (Mato Grosso). Types o VMNH \& SMF*. N. COMB.

viridula Bigot, 1875:481 (Volucella; preocc. Walker, 1860). Typelecality: "Mexico". Type o OXF*.

musta Williston, 1888:274 (Volucella). Type-locality: Brazil, Mato Grosso, Chapada. Types ơ AMNH*.

VAleria (Hull), 1944j:48 (Volucella). Type-locality: Peru, El Campamiento. Distr. - same. Type \& CU. N. COMB.

vaMPyRum (Hull), 1942g:96 (Volucella). Type-locality: Brazil, Paraná, Paranaguá. Distr. - same. Type ơ MCZ*. N. COMB.

VARIANS (Bigot), 1875:481 (Volucella). Type-locality: "Mexico". Distr. same. Type o $\mathrm{OXF}^{*}$. N. COMB.

VARICHAETUM (Curran), 1925b:250 (Phalacromyia). Type-locality: Peru, Río Pichis, Puerto Bermúdez. Distr. - same. Type $\sigma^{\circ} \mathrm{CU}$. N. COMB.

VARIEGatum (Bigot), 1875:478 (Volucella). Type-locality: "Mexico". Distr.

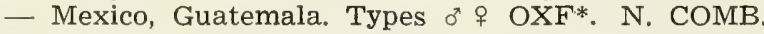

comastes Williston, 1891:52 (Volucella). Type-locality: Mexico, Orizaba. Types of $\mathrm{BM}(\mathrm{NH})$.

VENTANA (Fluke), 1951b:9, figs. 4 (head), 23 (scutellum), 37 (antenna), 50 (male genitalia) (Volucella). Type-locality: Argentina, Buenos Aires, Tornquist, Cerro de la Ventana. Distr. - same. Type $c^{2}$ IML. N. COMB.

NERA (Hull), 1942g:95 (Volucella). Type-locality: Honduras, Lancetilla. Distr. - same. Type o MCZ*. N. COMB.

VERDigaster (Hull), 1943k:41 (Volucella). Type-locality: Colombia, Bogotá. Distr. - same. Type o* USNM. N. COMB.

viciNum (Bigot), 1883b:86 (Phalachromyia). Type-locality: "Mexico". Distr. - same. Type $\sigma^{*} \mathrm{BM}(\mathrm{NH})$. N. COMB.

VIERECKI (Curran), 1925b:250 (Volucella). Type-locality: Colombia, Vista Nieve. Distr. - same. Type of AMNH*. N. COMB.

VILLARICA (Fluke), 1851b:23, figs. 12 (head), 62 (male genitalia) (Volucella). Type-locality: Paraguay, Villarica. Distr. - Brazil (São Paulo), Paraguay. Type \& AMNH*. N. COMB.

viola (Hull), 1944k:514 (Volucella). Type-locality: "Brazil". Distr. Brazil (São Paulo). Type \& Hull. N. COMB.

VIRESCENS (Williston), 1891:42 (Phalacromyia). Type-locality: Guatemala, E1 Tumbador, $2500 \mathrm{ft}$. Distr. - same. Type o BM(NH). N. COMB.

VIRIDANA (Townsend), 1897b:30 (Volucella). Type-locality: Mexico, Vera- 
cruz, Río Nautla, San Rafael. Distr. - same. Type ơ BM(NH). N. COMB.

VIRIDE (Williston), 1888:275 (Volucella). Type-locality: Brazil, Mato Grosso, Chapada. Distr. - Peru, Brazil (Mato Grosso). Types of o AMNH*. N. COMB.

VIRIDIGASTER (Hull), 1943k:41 (Volucella). Type-locolity: Ecuador. Distr. same. Type ơ USNM. N. COMB.

VIRIDULA (Walker), 1860:292 (Temnocera). Type-locality: "Mexico". Distr. - same. Type o BM(NH). N. COMB.

VIRTUOSA (Hull), 1942g:95 (Volucella). Type-locality: Paraguay, Villarica. Distr. - same. Type \& $\mathrm{MCZ}^{*}$. N. COMB.

vitrea (Hull), 1949h:36 (Volucella). Type-locality: Peru, Pucallpa Distr. - same. Type o Hull. N. COMB.

VITRIPENNE (Curran), 1930d:9, 1934b:387 (Volucella). Type-locality: Guyana, Kartabo. Distr. - same. Type of AMNH*. N. COMB.

VITTATUM Thompson (nom. nov, for americanum Goot). Distr. - Canada (Alberta), east to U.S.A. (New York); south to Mexico. Ref. Williston, 1887b:145 (redescription), pl. 6:4 (wing); Curran, 1925a:206, pl. 10:140 (male genitalia). NOM. NOV.

fasciatum Macquart, 1842:82 (22), pl. 5, fig. 2 (habitus) (Volucella; preocc. Herrich-Schaeffer, 1829). Type-locality: U.S.A., "Carolina". Type o MNHN*.

americanum Goot, 1964:214 (Volucella; nom. nov. for fasciatum Macquart, but preocc. Johnson, 1916).

vitTifacium (Hull), 1943d:18 (Volucella). Type-locality: Brazil, Amazon. Distr. - same. Type \& BM(NH). N. COMB.

VOLUCELloIDES (Bigot), 1884a:548 (Glaurotricha). Type-locality: "Mexico". Distr. - same. Type o $\mathrm{BM}(\mathrm{NH})$. N. COMB.

VOLUCRE (Giglio-Tos), 1892c:4 (Volucella). Type-locality: Mexico, Orizaba. Distr. - Mexico (San Luis Potosí, Veracruz). Type $0^{*}$ UTOR. N. COMB.

vUlCaN (Hull), 1942g:93 (Volucella). Type-locality: Brazil, Santa Catarina, Joinville. Distr. - same. Type o MCZ. N. COMB.

vulTA (Fluke), 1951b:6 figs. 17 (head), 22 (scutellum), 39 (antenna), 53 (male genitalia) (Volucella). Type-locality: Argentina, Salta, Cafayate. Distr. - Peru (Lima), Argentina (Tucumán, Salta). Type o IML. N. COMB.

WILLINKI (Fluke), 1951b:10, figs. 2 (head), 24 (scutellum), 54 (male gentialia) (Volucella). Type-locality: Argentina, Tucumán, Siambón. Distr. - Argentina (Tucumán, Buenos Aires). Type o IML. N. COMB.

WUlPI (Goot), 1964:214 (Volucella; nom. nov. for testaceum Wulp). Distr. - Venezuela. N. COMB.

testaceum Wulp, 1891:203 (Volucella; preocc. Rondani, 1848). Typelocality: "Venezuela; Curaçao". Types of \& ML? [not found JRV].

XIPE (Hull), 1942g:97 (Volucella). Type-locality: Panama, Chilibre. Distr. - same. Type of AMNH*. N. COMB.

YURA (Curran), 1930d:22 (Volucella). Type-locality: Peru, Yura. Distr. same. Type \& AMNH*. N. COMB.

zePHYRA (Curran), 1939b:5 (Volucella). Type-locality: Brazil, Santa Catarina, Nova Teutônia. Distr. - same. Type o AMNH*. N. COMB. 


\section{Tribe CALLICERINI}

\section{Genus Callicera Panzer}

Callicera Panzer, 1809:17. Type-species, Bibio aenea Fabricius (mon.). Ref. - Curran, 1935 (key).

Poultoni Verrall, 1913:332. Type-locality: "Mexico". Distr. - same. Type of $\mathrm{BM}(\mathrm{NH})$

\section{Genus Notiocheilosia Thompson}

Notiocheilosia Thompson, 1972:112. Type-species, Chilosia nitescens Shannon \& Aubertin (orig. des.).

NITESCENS (Shannon \& Aubertin), 1933:143 (Chilosia). Type-locality: Chile, Ensenada. Distr. - Chile (Santiago-Malleco), Argentina (Patagonia). Type o USNM*.

\section{Tribe SERYCOMYIINI}

\section{Genus ARctophila Schiner}

ARCTорнiLA Schiner, 1860:215. Type-species, Syrphus bombiformis Fallén (Williston, 1887:158).

Tapetomyia Fluke, 1939:370. Type-species, meyeri Fluke (orig. des.). N. SYN.

MEYERI (Fluke), 1939:370, figs. 8 (head), 9 (wing tip), 10 (hind leg) (Tapetomyia). Type-locality: Mexico, Mexico City, 10,000 ft. Distr. - same. Type o AMNH*. N. COMB.

\section{Tribe CHRYSOGASTERINI}

\section{Genus Lepidomyia Loew}

LEPIDOMYia Loew, 1864a:69 (1864b:231). Type-species, calopus Loew (mon.). Lepidostola Mik, 1886:278 (unjustified nom. nov. for Lepidomyia Loew). Lepromyia Williston, 1887:31 (unjustified nom. nov. for Lepidomyia Loew).

Ref. - Hull, 1946b (rev., key).

ABDOMINALlis (Williston), 1888:262 (Lepidostola). Type-locality: Brazil, Mato Grosso, Chapada. Distr. - Brazil. Type o AMNH*.

BRETHESI (Shannon), 1928a:573 (Lepidostola). Type-locality: Bolivia, "Prov.-Sara". Distr. - Bolivia (El Beni). Type o MACN.

Calopus Loew, 1864a:69 (1864b:231). Type-locality: "Cuba". Distr. - same. Type \& MCZ [lost]*. Ref. - Hull, 1946b:9 (redescription), figs. $2 \& 11$ (head), 18 (wing), 27 (mesonotum), 37 (abdomen).

Colombia (Curran), 1929:495 (Lepidostola). Type-locality: Colombia, Río Frío, Magdalena. Distr. - same. Type o AMNH.

CREPUSCUSTELlata (Hull) 1946b:4, figs. 3 (head), 16 (wing), 19 (mesonotum), 28 (abdomen) (Lepidostola). Type-locality: Brazil, Santa Catarina, Nova Teutônia. Distr. - same. Type o* AMNH*.

DIONYSIANA (d'Andretta \& Carrera), 1952:300, figs. 4 \& 5 (middle \& hind legs), 6 (wing), 7 (mesonotum), 8 (abdomen) (Lepidostola). Type-locality: Brazil, Acre. Distr. - same. Type + MZUSP*.

JENNINGSI (Shannon), 1925a:108 (Lepidostola). Type-locality: Panama, Canal Zone. Distr. - Panama, Brazil (Santa Catarina. Type $\sigma^{*}$ 
USNM*. Ref. - Hull, 1946b:12 (redescription), figs. 6, 10 (head), 21 (mesonotum), 35 (abdomen).

ortalina Wulp, 1888:374. Type-locality: Argentina, Córdoba. Distr. Brazil (Mato Grosso), Argentina (Tucumán, Córdoba, Buenos Aires). Type o lost?. Ref. - Hull, 1946b:8 (redescription), figs. 7 (head), 14 (wing), 25 (mesonotum), 30 (abdomen).

var. LACTIVA (Hull), 1946b:9 (Lepidostola). Type-locality: Brazil, Mato Grosso, Chapada. Type ? AMNH*.

PERPolita (Johnson), 1921:56 (Lepidostola). Type-locality: "Mexico". Distr. - same. Type o MCZ.

PULCHRA (Williston), 1888:261 (Lepidostola). Type-locality: Brazil, Mato Grosso, Chapada. Distr. - Brazil (Mato Grosso, Acre). Types ơ $q$ AMNH.

SIMILIS (Williston), 1888:262 (Lepidostola). Type-locality: Brazil, Mato Grosso, Chapada. Distr. - Peru (Madre de Dios), Brazil (Mato Grosso, Santa Catarina, São Paulo). Type \& AMNH. Ref: - Hull, 1946b:5 (description of male), figs. 1 (head), 15 (wing), 26 (mesonotum), 29 (abdomen).

Stellata (Hull), 1941a:1 (Lepidostola). Type-locality: Brazil, São Paulo. Distr. - same. Type o AMNH. Ref. - Hull, 1946b: 12, figs. 8 (head), 13 (wing), 24 (mesonotum), 33 (abdomen).

TRILIIEEATA (Hull), 1941a:2 (Lepidostola). Type-locality: Brazil, Mato Grosso, Poxoréu, Coronel Ponce. Distr. - Peru (Madre de Dios), Brazil (Mato Grosso). Type o AMNH. Ref. - Hull, 1946b:12, figs. 5 \& 9 (head), 27 (mesonotum), 36 (abdomen).

vUlturella (Hull), 1946b:7, figs. 4 (head), 17 (wing), 23 (mesonotum), 34 (abdomen) (Lepidostola). Type-locality: Brazil, Santa Catarina, Nova Teutônia. Distr. - Brazil (Santa Catarina), Argentina (Tucumán). Type $\sigma^{\star}$ AMNH.

\section{Genus Myolepta Newman}

MyolePta Newman, 1838:373. Type-species, Musca luteola Gmelin (mon.). Myiolepta, emendation.

Xylotaeja Rondani, 1845:457. Type-species, Syrphus vara Panzer (orig. des.).

Eumyiolepta Shannon, 1921:71. Type-species, Myolepta strigilata Loew (orig. des.).

Sarolepta Hull, 1941i:436. Type-species, dolorosa Hull (orig. des.). Ref. - Fluke \& Weems, 1956 (rev., keys).

\section{Subgenus Myolepta Newman}

AURICAUDATA Williston, 1891:40, pl. 1:11 (habitus), 11a (head), 11b (hind leg). Type-locality: Mexico, Guerrero, Xucumanatlán; Morelos, Cuernavaca. Distr. - U.S.A. (Arizona); Mexico (Guerrero, Morelos). Type of $q \mathrm{BM}(\mathrm{NH})$. Ref. - Fluke \& Weems, 1956:11 (redescription), figs. 6 (mesonotum), 15 (antenna).

Dolorosa (Hull), 1941i:436 (Sarolepta). Type-locality: "Venezuela". Distr. - same. Type o VMNH.

GREENI Hull, 1941i:435. Type-locality: Argentina, Tucumán, Villa Nougés. Distr. - same. Type o USNM. Ref. - Fluke \& Weems, 1956:10 (redescription), figs. 12 (antenna), 29 (male genitalia).

schreiteri Blanchard, in Fluke \& Weems, 1956:11. Nomen nudum. 
HAEMORRHOIDALIS (Philippi), 1865:740 (Priomerus?). Type-locality: Chile, Santiago. Distr. - Chile, Argentina (Neuquén). Type ? MNHNS. Ref. - Fluke \& Weems, 1956:8 (redescription), fig. 9 (antenna). LUCTUOSA (Bigot), 1857a:296, pl. 7:7 (wing, head, antenna) (Helophilus). Type-locality: "Chile". Distr. - same. Type o BM(NH). Ref. Fluke \& Weems, 1956:7 (description), fig. 13 (antenna).

luctuosa Philippi, 1865:739 (Priomerus?; preocc. Bigot, 1857). Typelocality: Chile. Type ? MNHNS.

NAUSICAA (Hull), 1937a:172, fig. 3 (mesonotum) (Nausigaster). Typelocality: Uruguay, Montevideo. Distr. - Uruguay, Argentina (Formosa), Chile (Santiago). Type o USNM*. N. COMB.

apicalis Fluke, in Fluke \& Weems, 1956:9, figs. 3 (head), 5 (thorax \& abdomen), 10 (antenna), 27 (male genitalia). Type-locality: Argentina, Formosa, Juarez, Ingenio. Type ơ IML. N. SYN.

\section{Subgenus Protolepidostola Hull}

PRotolepidostola Hull, 1949d:333 (as genus). Type-species, Lepidostola scintillans Hull (orig. des.)

ERAZILIANA (Shannon), 1927a:14 (Eumyiolepta). Type-locality: Brazil, Teresópolis. Distr. - same. Type o $\mathrm{BM}(\mathrm{NH})$.

?CIRCUlaris (Hull), 1941i:434 (Eumyiolepta). Type-locality: Argentina, Tucumán, Villa Nougés. Distr. - same. Type \& USNM. Ref. Fluke \& Weems, 1956:12 (description), figs. 11 (antenna), 30 (male genitalia).

EVANSI Thompson, 1968:276. Type-locality: Mexico, Guerrero, $16 \mathrm{~km}$ east of Chilpancingo, $4700 \mathrm{ft}$. Distr. - same. Type i MCZ*.

MiNuTA Fluke, in Fluke \& Weems, 1956:16, figs. 17 (wing tip), 32 (male genitalia). Type-locality: Argentina, Tucumán, Tafí, Locavera. Distr. - same. Type o IML.

Pfioblematica Thompson, 1968:275, figs. 2, 2a \& 2b (head). Type-locality: Panama, Canal Zone, Barro Colorado. Distr. - same. Type \& $\mathrm{MCZ}$ *

Scintillans (Hull), 1946b:3, figs. 12 (head), 20 (mesonotum), 31 (abdomen) (Lepidostola). Type-locality: Brazil, Santa Catarina, Nova Teutônia. Distr. - same. Type o $\mathrm{AMNH}^{*}$.

\section{Genus Chromocheilosia Hull \& Fluke}

Cheilosia, subg. CHROMocheiLosia Hull \& Fluke, 1950:306. Type-species, Chilosia bicolor Shannon \& Aubertin (orig. des.). Ref. - Shannon \& Aubertin, 1933:141 (key).

BICOLOR (Shannon \& Aubertin), 1933:141, fig. 27a (head) (Chilosia). Type-locality: Chile, Casa Pangue. Distr. - same. Type o $\mathrm{BM}(\mathrm{NH})$.

INCERTA (Shannon \& Aubertin), 1933:142, fig, 27b (head) (Chilosia). Type-locality: Chile, Casa Pangue. Distr. - Chile. Type o USNM*. PUBESCENS (Shannon \& Aubertin), 1933:142 (Chilosia). Type-locality: Chile, Casa Pangue. Distr. - Chile, Argentina (Neuquén). Type $\sigma^{\circ}$ $\mathrm{BM}(\mathrm{NH})$.

\section{Genus Orthonevra Macquart}

ORTHONEVRA Macquart, 1829:188 (40). Type-species, Chrysogaster elegans Meigen (mon.).

Orthoneura, emendation. 
Cryptineura Bigot, 1859:308. Type-species, hieroglyphica Bigot (mon.) $=$ nitida Wiedemann.

Chrysogaster authors (part).

Ref. - Sedman, 1964 \& 1966 (rev., keys).

?ANNUlifera Bigot, 1884a:556. Type-locality: "Brazil". Distr. - same, Type o OXF.

ARGENTINA (Brèthes), 1922:141 (Chrysogaster). Type-locality: Argentina, Buenos Aires, Sierra de la Ventana. Distr. - same. Type o MACN. N. COMB.

BELLULA (Williston), 1882:304 (Chrysogaster). Type-locality: U.S.A., Washington Territory, California. Distr. - Western U.S.A.; ?Mexico (Durango, Michoacán) [Williston, 1891:7]. Types o f USNM*. Ref. - Sedman, 1964:170 (description), figs. 4 (eye markings), 11 (mesonotum), 16 (wing). N. COMB.

FLUKEI (Sedman), 1964:173, figs. 3 (male genitalia), 8 (eye markings), 10 (mesonotum), 12 (wing) (Chysogaster). Type-locality: U.S.A., Arizona, Chochise Co., $17 \mathrm{mi}$. east of Douglas. Distr. - U.S.A. (California to Nevada), south to Mexico (Baja California \& Chiapas). Type o UCD*. N. COMB.

GEWGAW (Hull), 1941g:152, fig. 11 (wing) (Chrysogaster). Type-locality: Cuba, Soledad, Distr. - same. Type or MCZ*. N. COMB.

LABYRINTHOPS (Hull), 1944j:44 (Chrysogaster). Type-locality: Argentina, Mendoza, Potrerillos, $4000 \mathrm{ft}$. Type or CU. N. COMB.

?LUGUBRIS (Jaennicke), 1867:404 (Chrysogaster). Type-locality: Chile. Distr. - same. Type o SMF. N. COMB.

NEOTROPICA (Shannon), 1925a:107 (Chrysogaster). Type-locality: Paraguay, San Bernardino. Distr. - same. Type of USNM. N. COMB.

nitida (Wiedemann) not neotropical [Sedman, 1964:176].

QUADRISTRIATA (Shannon \& Aubertin), 1933:140 (Chrysogaster). Type-locality: Argentina, Neuquén). Type ơ USNM. N. COMB.

SHANNONI (Curran), 1925a:119 (Chrysogaster). Type-locality: Costa Rica, San Mateo, Huigito. Distr. - same. Type o USNM. N. COMB.

\section{Genus Cacoceria Hull}

Cacomyia Hull, 1930:147 (preocc. Coquillett, 1906). Type-species, cressoni Hull (orig. des.).

CACOCERIA Hull, 1936a:227 (nom. nov. for cacomyia Hull).

CRESSONI (Hull), 1030:147, pl. 9:1 (mesonotum), 3 (hind leg), 4 (head) (Cacomyia). Type-locality: "Mexico". Distr. - same. Type \& ANSP.

Willistoni Hull, 1949i:225. Type-locality: Peru, Chanchamayo. Distr. Peru, Argentina (Tucumán). Type ơ Hull.

\section{Genus Chamaesphegina Shannon \& Aubertin}

CHAMAESPHEgina Shannon \& Aubertin, 1933:145. Type-species, argentifacies Shannon \& Aubertin (orig. des.)

Desmetrum Enderlein, 1938:193. Type-species, macrum Enderlein (orig. des.).

ARGENTIFACIES Shannon \& Aubertin, 1933:145, figs. 28 (head), 29 (wing). Type-locality: Argentina, Ensenada. Distr. - Argentina, Chile (Arauco, Aysén, Curicó, Malleco). Type ơ $\mathrm{BM}(\mathrm{NH})$.

MACrA (Enderlein), 1938:194 (Desmetrum). Type-locality: Middle Chile, "Comudes". Distr. - same. Type o ZMB. 


\section{Genus Brachyopa Meigen}

BRACYOPA Meigen, 1822:260. Type-species, Musca conica Panzer (Westwood, 1840:137) = panzeri Goffe.

Unnamed sp. - Mexico (Mexico).

\section{Tribe EUMERINI}

\section{Genus Eumerus Meigen}

EUMERUS Meigen, 1822:202. Type-species, Syrphu; tricolor Fabricius (Curtis, 1839:pl. 749). Ref. - Latta \& Cole, 1933 (rev., key).

TUBerculatus Rondani, 1857:93. Type-locality: "Italy". Distr. - Europe, Canada, U.S.A.; Colombia (Magdalena). Types o o MZUF*. Ref. - Collin, 1920:103 (redescription), figs. 1 (4th sternum of male), 3 (hind leg), 4-6 (male genitalia).

\section{Genus Alipumilio Shannon}

ALIPUMilio Shannon, 1927a:12. Type-species, femoratus Shannon (orig. des.). Ref. - Vockeroth, 1964 (key).

AVISPAS Vockeroth, 1964:924, fig. 3 (wing tip). Type-locality: Peru, Madre de Dios, Avispas, $400 \mathrm{~m}$. Distr. - same. Type o CNC*.

FEMøratus Shannon, 1927a:12, figs. 1 (wing), 2 (head), 3 (head). Typelocality: "Amazon". Distr. - same. Type \& BM(NH)*. Ref. Hull, 1949d:336, figs. 16a (habitus), 16b (hind leg), 16c,d (head).

NIGRoCoERULEUS Vockeroth, 1964:924, fig. 2 (wing tip). Type-locality: Mexico, Morelos, Yautepec, Canyon de Lobos, $4000 \mathrm{ft}$. Distr. same. Type \& CU*.

PULlatus Vockeroth, 1964:923, figs. 1 (wing tip), 4 (head). Type-locality: Peru, Madre de Dios, Avispas, $400 \mathrm{~m}$. Distr. - same. Type $\&$ CNC*.

\section{Genus NAUSigaster Williston}

NAUSIGaSTER Williston, 1883:33. Type-species, punctulata Williston (mon.). Ref. - Curran, 1941 (key); Carrera, Lopes, \& Lane, 1947b (biol., immature stages).

BONARIENSIS Lynch Arribálzaga, 1892d:254 (1892c:155). Type-locality: Argentina, Buenos Aires. Distr. - Argentina, Brazil (north to Mato Grosso \& Rio de Janeiro). Type o MACN.

shannoni Carrera, Lopes \& Lane, 1947b:481, figs. 28 (wing), 29-38 (male genitalia + abdomen), 39-49 (larva). Type-locality: Brazil, Rio de Janeiro. Type $\sigma^{*}$ IOC. N. SYN.

CHRYSIDIFORMIS Shannon, 1922b:39. Type-locality: Peru, Río Charape. Distr. - same. Type $\%$ USNM.

FLUKeI Curran, 1941:256. Type-locality: Paraguay, Villarica. Distr. Brazil (São Paulo, Paraná), Paraguay. Type of AMNH*. Ref. Carrera, et. als, 1947b:478 (description of male), figs. 20 (wing), 21-26 (male genitalia).

Gemrnata Townsend, 1897d:25. Type-locality: U.S.A., Texas, Beeville \& Kenedy. Distr. - U.S.A. (Texas, Arizona), Mexico (Sonora, Durango, Hidalgo). Types of $\mathrm{BM}(\mathrm{NH}) *$ \& VMNH*.

MERIDIONALIS Townsend, 1897a:20. Type-locality: Mexico, Vera Cruz, San Rafael. Distr. - Mexico (Vera Cruz), ?Brazil (Curran, 1941: 255). Type \& $\mathrm{BM}(\mathrm{NH}) *$.

PERUVIENSIS Shannon, 1922a:40. Type-locality: Peru, Santa Eulalia. Distr. - same. Type \& USNM. 
PUNCTATA Williston, 1883:34, pl. 2, fig. 15 (habitus). Type-locality: U.S.A., New Mexico. Distr. - U.S.A. (California to Texas); Mexico (Durango), ?Brazil (Williston, 1888:259). Type of USNM.

tuberculata Carrera, Lopes \& Lane, 1947b:480, fig. 27 (wing). Typelocality: Brazil, Paraná, Iguaçu. Distr. - Brazil (Paraná, Santa Catarina). Type o IOC.

Unimaculata Townsend, 1897b:24. Type-locality: U.S.A., California. Distr. - U.S.A. (California to Texas); Mexico (Sonora). Types $\sigma^{7}$ $\mathrm{BM}(\mathrm{NH})$ \& USNM.

vANZOLINII d'Andretta \& Carrera, 1952:298, figs. 2 (wing), 3 (abdomen). Type-locality: Brazil, Acre. Distr. - same. Type $q$ MZUSP*.

\section{Tribe CERIOIDINI}

\section{Genus SpHIXIMORPHA Rondani}

SPHIXIMORPHA Rondani, 1850c:212. Type-species, Ceria subsessilis Illiger (orig. des.)

Sphyximorpha, emendation

Cerioides Rondani, 1850c:211 (originally cited in synonymy; validated by Becker, et al., 1907:156). Type-species, Ceria subsessilis Illiger (aut.)

Ceriodes, misspelling.

Cerioides, subg. Polistoceria Hull, 1949d:380. Type-species, kerteszi Shannon (orig. des.).

Ref. - Curran, 1941 (key).

Barbipes (Loew), 1853:19 (Ceria). Type-locality: Uruguay, Montevideo. Distr. - Brazil (São Paulo, Rio de Janeiro, Mato Grosso, Santa Catarina), Uruguay, Argentina. Type o ZMB. N. COMB.

acra Curran, 1941:246 (Cerioides), Type-locality: Brazil, Santa Catarina, Nova Teutônia. Type o AMNH*. N. SYN.

BIGoTII (Williston), 1888:291 (Ceria). Type-locality: Brazil, Mato Grosso, Chapada. Distr. - Brazil (São Paulo, Rio de Janeiro, Mato Grosso). Types of o AMNH*. N. COMB.

Boliviana (Kertész), 1903:434 (Ceria). Type-locality: Bolivia, Songo. Distr. - same. Type o UNMB [destroyed]. N. COMB.

BRAUERII (Williston), 1888:289 (Ceria). Type-locality: Brazil, Mato Grosso, Chapada; Pará, Santarém. Distr. - Peru, Brazil (Pará, Mato Grosso), Bolivia. Types o AMNH*. Ref. - Hull, 1949d:267, fig. 4d (abdomen). N. COMB.

var. PHILIPPI (Shannon), 1925c:63 (Cerioides). Type-locality: Bolivia, Ivon, Beni. Type $\&$ USNM.

Facialis (Kertész), 1903:439 (Ceria). Type-locality: "Paraguay". Distr. Brazil (Paraná, Santa Catarina), Paraguay. Type o" UNMB [destroyed]. N. COMB.

Flavosignata (Kertész), 1902:89 (Ceria). Type-locality: Bolivia, Songo; Peru, Maracapota, $1000 \mathrm{~m}$. Distr. - same. Types o UNMB [destroyed]. N. COMB.

KERTESZI (Shannon), 1925c:63 (Cerioides). Type-locality: Peru, Chanchamayo. Distr. - same. Type o USNM. N. COMB.

MEADEI (Williston), 1892:76, pl. 2:12 (habitus), 12a $+\mathrm{b}$ (head) (Ceria). Type-locality: Mexico, Guerrero, Xucumanatlán, $7000 \mathrm{ft}$. Distr. same. Types o $\mathrm{BM}(\mathrm{NH})$. N. COMB.

MIKII (Williston), 1888:288 (Ceria). Type-locality: Brazil, Mato Grosso, Chapada. Distr. - same. Types of AMNH*. N. COMB. 
NIGRIPENNIS (Williston), 1887:263 (Ceria). Type-locality: Mexico. Distr. same. Type o $\mathrm{MCZ}^{*}$. N. COMB.

PICTA (Kertész), 1902:87 (Ceria). Type-locality: Bolivia, Songo; Peru, Callanga. Distr. - same. Types o of UNMB [destroyed] N. COMB.

POLISTES (Curran), 1941:246 (Cerioides). Type-locality: Brazil, Santa Catarina, Nova Teutônia. Distr. - Brazil (Santa Catarina), Paraguay. Type o AMNH*. N. COMB.

?POLISTIFORMIS (Hull), 1944i:404 (Cerioides). Type-locality: Bolivia, Cuesta de Sillunticara, $3000 \mathrm{~m}$. Distr. - same. Type o VMNH. N. COMB.

PYRRHOCERA (Kertész), 1903:435 (Ceria). Type-locality: Bolivia, S. Antonio. Distr. - same. Type + UNMB [destroyed]. N. COMB.

ROEDERII (Williston), 1888:289 (Ceria). Type-locality: Brazil, Mato Grosso, Chapada. Distr. - Peru, Brazil (Mato Grosso). Type o AMNH*. N. COMB.

?RUBRoBrunNeA (Hull), 1944i:403 (Cerioides). Type-locality: Colombia, Muzo. Distr. - same. Type o VMNH. N. COMB.

SACKENII (Williston), 1888:287 (Ceria). Type-locality: Brazil, Mato Grosso, Chapada. Distr. - Brazil (Mato Grosso), Argentina (Formosa, Tucumán). Types o* AMNH*. N. COMB.

SHANNONI (Lane \& Carrera), 1943:169 (Cerioides). Type-locality: Brazil, São Paulo, Juquiá. Distr. - same. Type ơ MZUSP. N. COMB.

Signifera (Loew), 1853:18 (Ceria). Type-locality: "Mexico". Distr. U.S.A. (Minnesota to Massachusetts, south to Florida); Mexico. Type \& ZMB? N. COMB.

SuPERBA (Williston), 1887:264 (Ceria). Type-locality: "Mexico". Distr. same. Type $\sigma^{*} \mathrm{MCZ}^{*}$. N. COMB.

TRICHOPODA (Kertész), 1903:438 (Ceria). Type-locality: Bolivia, Songo. Distr. - same. Type $\sigma^{*}$ UNMB [destroyed]. N. COMB.

VARIABILIS (Kertész), 1903:437 (Ceria). Type-locality: Bolivia, Songo. Distr. - Bolivia, Peru. Type of UNMB [destroyed]. N. COMB.

vicina (Kertész), 1902:89 (Ceria). Type-locality: Peru, Cagon, $1000 \mathrm{~m}$. Distr. - same. Type o UNMB [destroyed]. N. COMB.

WULPII (Williston), 1888:290 (Ceria). Type-locality: Brazil, Mato Grosso, Chapada; Rio de Janeiro. Distr. - Brazil (Rio de Janeiro, São Paulo, Mato Grosso). Types o o AMNH*. Ref. - Sack, 1921:149, fig. 25 (puparium). N. COMB.

\section{Genus Monoceromyia Shannon}

MONOCEROMYia Shannon, 1922d:41. Type-species, Ceria tricolor Loew (mon.).

BICoLor (Kertész), 1902:86 (Ceria). Type-locality: Peru, Callanga. Distr. Peru, Bolivia. Type \& UNMB [destroyed]. N. COMB.

CACICA (Walker), 1860:288 (Ceria). Type-locality: "Mexico". Distr. - same. Type of $\mathrm{BM}(\mathrm{NH})$. N. COMB.

DAPHNAEuS (Walker), 1849:537 (Ceria). Type-locality: "Jamaica". Distr. - same. Type o $\mathrm{BM}(\mathrm{NH})$. N. COMB.

LYNCHII (Williston), 1888:287 (Ceria). Type-locality: Brazil, Mato Grosso, Chapada. Distr. - same. Type AMNH*. N. COMB.

TriColor (Loew), 1861:37 (Ceria). Type-locality: "Cuba". Distr. - Cuba. Types of o MCZ. Ref. - Hull, 1949d:264, fig. 3b (head).

verralli (Williston), 1892:75 (Ceria). Type-locality: Panama, David. Distr. - same. Type or BM(NH). N. COMB.

vittipes (Curran), 1941:246 (Cerioides). Type-locality: Peru, Puerto Bermúdez. Distr. - same. Type o AMNH*. N. COMB. 


\section{Genus Polybiomyia Shannon}

polybiomyia Shannon, 1925c:56. Type-species, schwarzi Shannon (orig des.). Ref. - Shannon, 1925c (key), Curran, 1941 (key, in part).

Arietis (Loew), 1853:17 (Ceria). Type-locality: "Mexico". Distr. - same. Type o ZMB.

BASSLERI (Curran), 1941:245 (Cerioides). Type-locality: Peru, Upper Río Huallaga. Distr. - same. Type o AMNH*. N. COMB.

BERGRothi (Williston), 1892:77 (Ceria). Type-locality: Mexico, Vera Cruz, Atoyac. Distr. - same. Type o $\mathrm{BM}(\mathrm{NH})$.

CAPITIS (Curran), 1925a:27, pl. 1:2 (head) (Cerioides). Type-locality: Mexico, Mexico City. Distr. - U.S.A. (Texas); Mexico (Distrito Federal). Type ot USNM.

LYNCHARRIBALZAGaI Shannon, 1927d:40. Type-locality: Argentina, Tucumán, Quebrada de Lules. Distr. - same. Type + MACN?

MACQUARTI Shannon, 1925c:59 (nom. nov. for scutellata Williston). Distr. - U.S.A. (Texas); Mexico.

scutellata Williston, 1887:265 (Ceria; preocc. Macquart, 1842). Typelocality: Mexico, Type o USNM.

NIGRA (Bigot), 1883b:317 (Sphyximorpha). Type-locality: "Mexico". Distr. - same. Types of $\&$ BM(NH).

ODONTOMERA (Curran), 1941:244 (Cerioides). Type-locality: Brazil, Mato Grosso, Chapada. Distr. - Brazil (São Paulo, Acre, Mato Grosso). Type o AMNH. [lost-FCT]. N. COMB.

PEdiCEllata (Williston), 1887:264 (Ceria). Type-locality: "Mexico". Distr. - U.S.A. (Arizona, Texas, Florida); Mexico (Guanajuato, Isthmus of Tehuantepec). Type or USNM. Ref. - Curran, 1925a:30 (redescription), pl. 2:3 (head).

Plaumanni (Curran), 1941:245 (Cerioides). Type-locality: Brazil, Santa Catarina, Nova Teutônia. Distr. - same. Type $\delta^{*} \mathrm{AMNH}^{*}$. N. COMB.

RUFIBaSis (Bigot), 1883b:318 (Sphyximorpha). Type-locality: "Mexico". Distr. - same. Type o $\mathrm{BM}(\mathrm{NH})$.

SCHNABLEI (Williston), 1892:76 (Ceria). Type-locality: Mexico, Vera Cruz, Atoyac. Distr. - U.S.A. (Texas), Mexico (Vera Cruz). Types $\sigma^{*}$ $\mathrm{BM}(\mathrm{NH})$.

SCHWARzi Shannon, 1925c:57. Type-locality: Panama, Canal Zone, Barro Colorado Island. Distr. - same. Type o USNM.

TownSENdr (Snow), 1895b:246 (Ceria). Type-locality: U.S.A., New Mexico, Las Cruces. Distr. - U.S.A. (California to Texas); Mexico (Durango). Type or UK.

TRAVASSOSI (Lane \& Carrera), 1943:172 (Cerioides). Type-locality: Brazil, São Paulo, Rio Paraná, Porto Cabral. Distr. - same. Type o MZUSP*. N. COMB.

\section{UNPLACED SPECIES OF CERIOIDINI}

NITIDA Sack, 1941:118 (1951:114) (Cerioides). Type1locality: Peru, Umachuán. Types ơ $q$ ?

RกBUSTA Sack, 1941:119 (1951:115) (Cerioides). Type-locality: Peru, mouth of Pachitea River; Meshagua on Urubamba River. Types ' ? 


\section{Tribe ERISTALINI}

\section{Genus HeLophilus Meigen}

HeLophilus Meigen, 1822:368. Type-species, Musca pendula Linnaeus (Curtis, 1832:pl. 429). Ref. - Curran \& Fluke, 1926:211-229 (key, rev.).

Fasciatus Walker, 1849:605. Type-Iocality: Canada, Ontario, Hudson's Bay, Albany River, St. Martin's Fall. Distr. - Canada (British ,Columbia to Quebec), south to U.S.A. (Florida), Mexico; Haiti? [Walker, 1860]. Type BM(NH). Ref. - Curran \& Fluke, 1926:220 (redescription), pl. 5:6 (head).

similis Macquart, 1842:124 (64) (preocc. Curtis, 1832). Type-locality: Georgia. Type or $\mathrm{OXF}^{*}$.

impositus Walker, 1860:289 (Eristalis). Type-locality: Haiti (error?). Type of $\mathrm{BM}(\mathrm{NH})$.

LATIFRons Loew, 1863:313 (1864b:197). Type-locality: U.S.A., Nebraska. Distr. - Alaska to Labrador, south to U.S.A. (Florida), Mexico. Types o o MCZ. Ref. - Curran \& Fluke, 1926:216 (redescription), pl. 5:7, 8 (head).

\section{Genus Polydontomyia Williston}

Polydonta Macquart, 1850:448 (144) (preocc. Fischer, 1807). Type-species, bicolor Macquart (orig. des.) = albiceps (Macquart).

Triodonta Williston, 1885:136 (nom. nov, for Polydonta Macquart; preocc. Bory, 1827). Type-species, Polydonta bicolor Macquart (aut.) = albiceps (Macquart).

PoLydontomyia Williston, 1896:89. Type-species, Merodon curvipes Wiedemann (Coquillett, 1910a:593) = albiceps (Macquart).

ALBICEPS (Macquart), 1846:260 (132) (Helophilus). Type-locality: Canada, Nova Scotia. Distr. - Alaska, south to U.S.A. (California \& Nebraska); also Canada (eastern Quebec to Nova Scotia), south to U.S.A. (New Jersey); Mexico? [Wirth, et al., 1965:621], Brazil? [Walker, 1852:243]. Type क MNHN? N. STATUS.

curvipes Wiedemann, 1830:149 (Merodon; preocc. Gmelin, 1790). Typelocality: "North America". Type \& VMNH. N. STATUS.

bicolor Walker, 1852:243 (Merodon; preocc. Macquart, 1850). Type locality: Brazil (error?). Type o $\mathrm{BM}(\mathrm{NH}) *$. N. SYN.

\section{Genus Asemosyrphus Bigot}

ASEMOSYRPHUS Bigot, 1882a:cxxviii. Type-species, oculiferus Bigot (Aldrich, 1933:168) = mexicanus (Macquart).

Dimorphomyia Bigot, 1885b:clxxiii. Type-species, calliphoroides Bigot (mon.) = mexicanus (Macquart) [see Aldrich, 1933: 168]. Ref. - Curran, 1939c (key).

ARquatus (Say), 1829:162 (Xylota). Type-locality: "Mexico". Distr. same. Type ? lost. N. COMB.

formalis Walker, 1849:603 (Helophilus). Type-locality: "Mexico". Type \& $\mathrm{BM}(\mathrm{NH}) *$. N. SYN.

bicolor Bigot, 1882a:cxxix. Type-locality: "Mexico". Type \& BM(NH). N. SYN.

impurus Giglio-Tos, 1892c:6. Type-locality: "Mexico". Type o UTOR*. olivaceus Giglio-Tos, 1892c:6. Type-locality: "Mexico". Type o UTOR*. arcuata, Aldrich, 1905:398, emend. 
MEXICANUS (Macquart), 1842:124 (64), pl. 11, fig. 2 (habitus), 2a (head) (Helophilus). Type-locality: "Mexico". Distr. - same. Types o" 우 OXF*.

oculiferus Bigot, 1882a:cxxviii. Type-locality: "Mexico". Type $q$ $\mathrm{BM}(\mathrm{NH})$.

nigroscutatus Bigot, 1882a:cxxviii. Type-locality: "Mexico". Type $q$ $\mathrm{BM}(\mathrm{NH})$.

flavocaudatus Bigot, 1882a:cxxviii. Type-locality: "Mexico". Types $q$ $\mathrm{BM}(\mathrm{NH})$.

calliphoroides Bigot, 1885b:clxxiii (Dimorphomyia). Type-locality: "Mexico". Type? OXF.

griseus Giglio-Tos, 1892c:6. Type-locality: Mexico, Tehuacán. Types o i UTOR*

POLYGRAMMUS (Loew), 1872a:85 (1872b:261) (Helophilus). Type-locality: U.S.A., California. Distr. - Canada (British Columbia) to U.S.A. (Montana, south to California \& Colorado); Mexico? [Wirth, et al., 1965:620]. Type ㅇ MCZ.

\section{Genus Dolichogyna Macquart}

DOLICFOGYNA Macquart, 1842:125 (65). Type-species, fasciata Macquart (orig. des.) $=$ chiliensis (Guérin-Mèneville). Ref. - Fluke, 1951a (rev., key).

\section{Subgenus DoLichogYNA Macquart}

ABRupta Hine, 1914:342. Type-locality: Peru, Arequipa. Distr. - Chile, Peru. Type o OhSU. Ref. - Fluke, 1951a:472 (redescription), figs. 4,5 (head), 23 (male genitalia).

CHILIENSIS (Guérin-Méneville), 1835:545, tab. 99, fig. 2 (habitus), 2a (head). 2b (antenna), 2c (hind leg) (Helophilus). Type-locality: "Chile". Distr. - Chile, Argentina (Jujuy, Neuquén). Type ? MNHN [lost? - not found, JRV]. Ref. - Fluke, 1951a:467 (redescription), figs. 1,2 (head), 11, 12 (abdomen), 19, 22 (male genitalia).

chitensis, various authors, misspelling.

chilensis Walker, 1837:344 (Helophilus; preocc. Guérin-Mèneville, 1835). Type-locality: Chile, Chiloé. Types ơ o BM(NH).

fasciata Macquart, 1842:126, (186), tab. 12:1 (habitus) (66). Typelocality: Chile. Types o MNHN*.

hahni Bigot, 1888b:24, tab. 3:6 (habitus) (Helophitus). Type-locality: ?Cap Horn? [no definite locality given]. Type o o BM(NH).

MULLERI Fluke, 1951a:470, figs. 3 (head), 13 (abdomen), 15 (male genitalia). Type-locality: Ecuador, Azuay, Girón. Distr. - Peru, Ecuador. Type o AMNH*.

PERUANA Sack, 1941:114 (1951:110). Type-locality: Peru, Ocana; Arequipa. Distr. - Peru, Chile. Types ơ SMF*.

\section{Subgenus NosodePus Speiser}

NOSODEPUS Speiser, 1914:131 (as genus). Type-species, minotaurus Speiser (orig. des.).

Conocotamyia Fluke, 1951a:473. Type-species, Helophitus picta Philippi (orig. des.).

HINEI Fluke, 1951a:476, figs. 6, 10 (head), 21 (cercus), 25 (middle tarsus). Type-locality: Peru, Puno. Distr. - Bolivia, Peru. Type on OhSU. 
MINotaurus (Speiser), 1914:132, figs. (head, leg) (Nosodepus). Typelocality: Peru, Guaqui. Distr. - same. Type of [Wiesbaden?].

NIGRIPES Bigot, 1883b:346. Type-locality: "Chile". Distr. - Argentina, Chile. Types of o BM(NH). Ref. - Fluke, 1951a:477 (redescription), figs. 16 (male genitalia), 26 (middle tarsus).

PENNIPES (Sack), 1941:114 (1951:110), fig. 7 (middle tarsus) (Tubifera). Type-locality: "Peru". Distr. - same. Types of o MD. N. COMB.

PICTA (Philippi), 1865:743 (Helophilus). Type-locality: "Chile". Distr. Peru, Argentina, Chile. Type o MNHNS. Ref, - Fluke, 1951a:474 (redescription), figs. 7 (head), 14 (abdomen), 20, 24 (male genitalia), 27 (middle tarsus).

REYNOLDSI Shannon \& Aubertin, 1933:165. Type-locality: Argentina, Tierra del Fuego, Río Grande. Distr. - Chile, Argentina. Type or BM(NH). Ref. - Fluke, 1951a:475 (redescription), figs. 8, 9 (head), 17, 18 (male genitalia).

ROSTRATA (Macquart), 1846:259 (131) (Helophilus). Type-locality: "Nouvelle-Grenade". Distr. - same. Type o $\mathrm{BM}(\mathrm{NH}) *$. N. COMB.

\section{Genus QUichuaNa Knab}

QUichuana Knab, 1913:13. Type-species, sylvicola Knab (orig. des.).

Quihuana, Hull, 1944k:510, misspelling.

Ref. - Hull, 1946a (rev., key).

ANGUSTRIVENTRIS (Macquart), 1855:110 (90), pl. 5, fig. 2 (head) (Merodon). Type-locality: unknown. Distr. - South America? Type o MNHN.

AURATA (Walker), 1857:153 (Helophilus). Type-locality: "Valley of Amazon". Distr. - Colombia, Peru. Type o BM(NH). Ref. - Hull, 1946a:13 (redescription), fig. 2 (head).

BEzzII Ceresa, 1934:387, figs. 3a+b (head), 3c (wing). Type-locality: Brazil, São Paulo, São Sebastião. Distr. - Brazil (São Paulo), Argentina (Tucumán). Types o o MCSNM*.

Borgmeieri Lane \& Carrera, 1944:206. Type-locality: Brazil, São Paulo, Horto Florestal da Cantareira. Distr. - same. Type o MZUSP*.

Calathea Shannon, 1925:111. Type-locality: Panama, Porto Bello. Distr. Panama, Colombia. Types of of USNM*. Ref. - Hull, 1946a:16, fig. 13 (head).

CESTUS Hull, 1946a:4, figs. 4 \& 14 (head), 16 (antenna). Type-locality: Venezuela, Mt. Duida. Distr. - same. Type of AMNH*.

Championi (Williston), 1892:69 (Mallota?). Type-locality: Mexico, Guerrero, Amula, $6000 \mathrm{ft}$. Distr. - Mexico (Durango, Veracruz, Guerrero). Type o $\mathrm{BM}(\mathrm{NH})$.' Ref. - Hull, 1946a:9 (redescription), figs. 19 (antenna), 27 (head).

nigra Giglio-Tos, 1892c:6 (Platynochaetus). Type-locality: Mexico, Orizaba. Types o UTOR*. N. SYN.

CINTA (Bigot), 1883b:345 (Lepidomyia). Type-locality: "Mexico". Distr. same. Type on BM(NH)*. Ref. - Hull, 1946a:16, figs, 21 (antenna), 26 (head).

DoLorosa Hull, 1946a:6. Type-locality: Bolivia, Mapiri. Distr. - same. Type $0^{\pi}$ AMNH*.

FASCIATA (Sack), 1941:115 (1951:111) (Myiatropa). Type-locality: Peru, Paso de Lares. Distr. - same. Types ot $+\mathrm{MD}+\mathrm{SMF}^{*}$.

IIERMoSA Hull, 1951b:67. Type-locality: Peru, Chanchamayo. Distr. - same. Type \& Hull.

INCA Shannon, 1925a:111. Type-locality: Peru, Huascaray. Distr. - Co- 
lombia, Peru. Type o USNM. Ref. - Hull, 1946a:16, figs. 11 \& 24 (head), 18 (antenna).

var. BREVICERA Hull, 1946a:7. Type-locality: Colombia, Atrata Valley, Boca de Arquia. Types $\sigma^{*} \mathrm{BM}(\mathrm{NH}) *$.

KNABI Shannon, 1927a:15. Type-locality: Bolivia, Beni, Cavinas. Distr. same. Type of USNM. Ref. - Hull, 1946a:16, figs. 9 \& 22 (head), 15 (antenna).

MontanA Hull, 1951b:65. Type-locality: Peru, Chanchamayo. Distr. Venezuela, Peru. Type or Hull.

NIGRICANS Thompson (nom. nov. for nigra Hull). Distr. - Peru. NOM. NOV.

nigra Hull, 1949g:133 (preocc. Giglio-Tos, 1892). Type-locality: Peru, Pucallpa. Type + Hull.

PARISII Ceresa, 1934:389, figs. $4 \mathrm{a}+\mathrm{b}$ (head), 4c (wing). Type-locality: Brazil, São Paulo, São Sebastião. Distr. - same. Types q MCSNM*.

PICADOI Knab, 1913:14. Type-locality: "Costa Rica". Distr. - Costa Rica, Colombia, Surinam. Type o USNM. Ref. - Hull, 1946a:16, figs. $12 \& 25$ (head), 17 (antenna).

Pogonosa Fluke, 1937:11, fig. 14 (head). Type-locality: Brazil, Santa Catarina, Nova Teutônia: Distr. - Venezuela, Brazil. Type ơ AMNH*. Ref. - Hull, 1946a:15, fig. 8 (head).

QUIXOTEA Hull, 1946a:14, fig. 6 (head). Type-locality: "Colombia". Distr. same. Types of of Hull.

RIESELI Shannon, 1927c:5. Type-locality: Argentina, Tucumán. Distr. same. Type o MACN?

SEPIAPENNIS Hull, 1944k:510 (Quihuana). Type-locality: Panama, Canal Zone, Barro Colorado Island. Distr. - same. Type o Hull. Ref. Hull, 1946a:15, figs. 1 \& 5 (head).

simonetta Hull, 1946a:5, figs. 3 \& 7 (head). Type-locality: Peru, Huancabamba. Distr. - same. Type o VMNH.

Subcostalis (Walker), 1860:291 (Xylota). Type-locality: "Mexico". Distr. - same. Type \& $\mathrm{BM}(\mathrm{NH})$. Ref. - Hull, 1946a:16, figs. 20 (antenna), 28 (head).

sylvicola Knab, 1913:14. Type-locality: Peru, Chanchamayo. Distr. same. Type \& USNM. Ref. - Hull, 1946a:16, figs. 10 \& 23 (head).

ursula Hull, 1949g:136. Type-locality: Peru, Pucallpa. Distr. - same. Type ơ Hull.

\section{Genus Mallota Meigen}

Mallota Meigen, 1822:377. Type-species, Syrphus fuciformis Fabricius (Rondani, 1845:452).

Imatisma Macquart, 1842:127 (67). Type-species, Eristalis posticatus Fabricius (orig. des.).

Ref. - Curran, 1940:12 (key).

ABERRANS Shannon, 1927a:17. Type-locality: Peru, Paltaybamba. Distr. same. Typa ㅇ USNM.

ANALIS (Macquart), 1846:260 (138), pl. 11, fig. 8 (habitus) (Imatisma). Type-locality: "Nova Grenada". Distr. - same. Type o BM(NH). BEQUAerti Hull, 1956:24. Type-locality: U.S.A., Texas, Chisos Mountains, Big Bend National Park. Distr. - U.S.A. (Texas, Arizona); Mexico. Type ơ MCZ. 
óLомвII Macquart, 1850:445 (141), tab. 13, fig. 3 (habitus), 3a (head), 3b (antenna), 3c (hind leg). Type-locality: Colombia. Distr. Colombia, Venezuela, Peru. Type of MNHN*.

vulpina Sack, 1941:116 (1951:112). Type-locality: Peru, Pichis. Types o + MD*. N. SYN.

apimima Hull, 1944i:402. Type-locality: "Venezuela". Type $\sigma^{*}$ VMNH*. N. SYN.

INTERMEdIA Hull, 1949g:125. Type-locality: Peru, Huánuco, F. Sinchono District. Distr. - same. Type of Fiull.

INVERSA Shannon, 1927a:16. Type-locality: Peru, Chanchamayo. Distr. Peru. Type o USNM.

MARGARITA Williston, 1892:70, pl. 2:7 (habitus), 7a (head), 7b (hind leg). Type-locality: Mexico, Guerrero, Omiltemi, 8000 ft.; Xucumanatlán, $7000 \mathrm{ft}$. Distr. - same. Types ơ o $\mathrm{BM}(\mathrm{NH})$ \& $\mathrm{AMNH}^{*}$.

mystacia Fluke, 1939:372, fig. 12 (head). Type-locality: Panama, Chiriquí, El Volcán. Distr. - same. Type o $0^{*} \mathrm{AMNH}^{*}$.

NIGRA Shannon, 1927a:16. Type-locality: Ecuador, Santa Inez. Distr. same. Type of BM(NH).

RUBiCUNDA Curran, 1940:13. Type-locality: Ecuador, Tungurahua, Mt. Tungurahua. Distr. - same. Type o AMNH*.

SACKENI Williston, 1882b:324. Type-locality: U.S.A., Washington Territory. Distr. - Western Canada \& U.S.A.; Mexico. Types $0^{\pi}$ USNM. Ref. - Williston, 1887b:204 (redescription), pl. 8:14 (wing).

S.ilti Curran, 1929:499. Type-locality: Colombia, Santa Marta, Vista Nieve. Distr. - same. Type o AMNH*.

SMITHI Williston, 1892:70, pl. 2:8 (habitus), $8 \mathrm{a}$ (head), 8b (antenna). Type-locality: Mexico, Guerrero, Omiltemi, $8000 \mathrm{ft}$. Distr. - same. Types on o $\mathrm{BM}(\mathrm{NH})$ \& $\mathrm{AMNH}$ *.

\section{Genus HabRomyia Williston}

HABROMYIA Williston, 1888:284. Type-species, coeruleithorax Williston (mon.).

Edwardsietta Hull, 1941i:437. Type-species, ochracea Hull (orig. des.).

Lycopale Hull, 1944h:129. Type-species, Meromacrus vittata Hull (orig. des.) = lizeri (Brèthes).

Criorthrix Hull, 1949d:391. Type-species, Habromyia rectilinea Hull (orig. des.).

BARBIELLINII (Ceresa), 1934:383, figs. 1 (habitus), 2a+b (head), $2 c$ (wing) (Quichuana). Type-locality: Brazil, São Paulo, São Sebastião. Distr. - same. Types ot o MCSNIM*.

CHrysotaenia Fluke, 1937:12, fig. 15 (abdomen). Type-locality: Brazil, Santa Catarina, Nova Teutônia. Distr. - same. Type o AMNH*.

COERUleithorax 'Williston, 1888:284. Type-locality: Brazil, Mato Grosso, Chapada. Distr. - Peru, Brazil. Types ơ o AMNH*. Ref. - Hull, 1949d:390, figs. 24a (wing), 24 j (head).

Flavifacies Shannon, 1927a:15. Type-locality: "Amazon". Distr. - same. Type or $\mathrm{BM}(\mathrm{NH}) *$.

FLoccula Hull, 1944j:47. Type-locality: Peru, Matucana, Distr. - same. Type o $\mathrm{CU}$.

LANGI Curran, 1934b:406. Type-locality: Guyana, Kamakusa. Distr. same. Type of AMNH*.

LIPOFlaVA Fluke, 1937:13. Type-locality: Peru, Yura. Distr. - Peru. Type o AMNH*. 
LIZERI (Brèthes), 1914:97 (Helophilus). Type-locality: Argentina, Córdoba. Distr. - Argentina. Type o MACN. N. COMB.

vittata Hull, 1937a:170 (Meromacrus). Type-locality: Argentina, Villa Nougés. Type † USNM*. N. SYN.

MAGNIFICA (Bigot), 1880a:85 (Plagiocera). Type-locality: Colombia. Distr. - same. Type ot OXF*. Ref. - Hull, 1949d:390, fig. 24h (head).

oCHRACEA (Hull), 1941i:437 (Edwardsietta). Type-locality: Panama, Canal Zone, Barro Colorado Island. Distr. - same. Type o USNM*. Ref. - Hull, 1949d:390, fig. 24f (head).

ReCtilinea Hull, 1942h:19. Type-locality: Bolivia, Río Songo, $800 \mathrm{~m}$. Distr. - Bolivia. Types of VMNH \& Hull.

XYLOTAEFORMIS (Schiner), 1868:360 (Mallota). Type-locality: "Chile". Distr. - same. Types of $\mathrm{VMNH}$.

\section{Genus ERISTALIS Latreille}

Tubifera Meigen, 1800:34. Type-species, Musca tenax Linnaeus (Coquillett, 1910a:618). Suppressed by I.C.Z.N., 1963:339.

Elophilus Meigen, 1803:274. Type-species, Musca tenax Linnaeus (Latreille, 1810:443).

ERISTALIS Latreille, 1804:194. Type-species, Musca tenax Linnaeus (Curtis, 1832:pl. 432). [In order to preserve established usage, we have followed Wirth, et al., 1965:622, in using Eristalis instead of its senior synonym, Elophilus.]

Eristaloides Rondani, 1845:453. Type-species, Musca tenax Linnaeus (Coquillett, 1910a:540).

Eristalomya Rondani, 1857:38. Type-species, Musca tenax Linnaeus (orig. des.).

Eriops Lioy, 1864:743 (preocc. Klug, 1808). Type-species, Musca tenax Linnaeus (Goffe, 1946:29).

Ref. - Hull, 1925a, 1925b (rev., key); Curran, 1930c, 1934b:407 (keys); Shannon \& Aubertin, 1933:159 (key to Chilean and Southern Patagonian spp.); Bean, 1949 (male genitalia, key).

\section{Subgenus ERISTALIS Latreille}

TENAX (Linnaeus), 1758:591 (Musca). Type-locality: "Europe". Distr. cosmopolitan. Type ? ? Ref. - Williston, 1887b:160 (redescription), pl. 7:7 (wing), 7a (head), 7b (antenna), 7c (hind leg).

\section{Subgenus EOSERISTALIS Kanervo}

EOSERISTALIS Kanervo, 1938:12. Type-species, Eristalis cerealis Fabricius (orig. des.).

AZTECUS Hull, 1935:326, figs. 5 (head), 7, 8 (hind femora). Type-locality: Mexico, Real del Monte. Distr. - same. Type + USNM*.

BELLARDII Jaennicke, 1867:400. Type-locality: "Mexico". Distr. - U.S.A. (Arizona); Mexico (Durango). Type o SMF. N. STATUS.

rufoscutata Bigot, 1880b:221 (Eristalomyia). Type-locality: Mexico. Types o $\%$ OXF*. N. SYN.

BOGoTENSIS Macquart, 1842:112 (52). Type-locality: Colombia, Santa Fé de Bogotá. Distr. - Colombia, Venezuela, Ecuador, Peru, Bolivia, Chile, Argentina (south to Tierra del Fuego). Types o MNHN* \& BM(NH). Ref, - Hull, 1925b:308 (redescription), pl. 11:7 (abdomen), 2:12 (head), 15 (mesonotum). 
assimilis Macquart, 1846:257 (129). Type-locality: "Colombia". Types $0^{\pi}$ o MNHN? (not found - JRV). N. SYN.

CIRCE Williston, 1891:59, pl. 2:3 (habitus), 3a (head). Type-locality: Mexico, Durango, Ciudad; Guerrero, Omiltemi, 8000 ft.; Jalapa. Distr. - Mexico (Durango-Chiapas). Types o $\$ \mathrm{BM}(\mathrm{NH})$. Ref. Hull, 1935:327 (redescription), fig. 2 (head).

bombusoides Giglio-Tos, 1892c:4. Type-locality: Mexico, Oaxaca. Types o* \& UTOR*.

GUADELUPENSIS Macquart, 1842:92 (32). Type-locality: "Guadeloupe" (Error?). Distr. - same. Type o MNHN*.

PERSA Williston, 1891:58. Type-locality: Mexico, Guerrero, Sierra de las Aguas Escondidas, $9000 \mathrm{ft}$. Distr. - same. Type o $\mathrm{BM}(\mathrm{NH}) *$

STIPATOR Osten-Sacken, 1877:336. Type-locality: U.S.A.: Colorado, Manitous Park \& Denver; New Mexico, Morino Valley; California. Distr. - Canada (British Colombia to Nova Scotia), south to U.S.A. (Florida), Mexico (Guerrero). Types o $\$$ MCZ. Ref. Williston, 1887b:164 (redescription).

latifrons Loew, 1866a:169 (1872b:43) (preocc. Fallén, 1817). Typelocality: Mexico, Matamoros. Types of o MCZ.

var. MACUlipennis Townsend, 1897d:93. Type-locality: U.S.A., New Mexico, Rio Gila, Head of East Fork. Types $0^{3}+\mathrm{B}_{\mathrm{B}}(\mathrm{NH})$.

TENUIFrons Curran, 1930c:12. Type-locality: Panama, Canal Zone, Fort Randolph. Distr. - same. Type o AMNH*.

\section{Genus LyCASTRIRHyNCHa Bigot}

LYCASTRIRHYNCHA Bigot, 1859:307. Type-species, nitens Bigot (mon.).

Lycastrirrhyncha, various authors, misspelling.

Ref. - Doesburg, 1962:31 (key).

Mexicana Curran, 1930e:15. Type-locality: Mexico, Vera Cruz. Distr. same. Type of AMNH*.

Nitens Bigot, 1859:307. Type-locality: "Amazonia". Distr. - Mexico (Veracruz), "Amazons". Type क OXF*. Ref. — Hull, 1949d:393, fig. 25b (habitus), 25m (head).

QUINTA Doesburg, 1963:31. Type-locality: Surinam, Paramaribo. Distr. same. Type of Doesburg*. Ref. - Doesburg, 1966:103 (description of male), figs. 67 (head), 68 (habitus).

TITILlans Hull, 1944j:53. Type-locality: Guyana, Demerara River, Mackenzie. Distr. - same. Type $0^{*}$ CU.

willistoni Coquillett, 1902:196. Type-locality: Mexico, Tabasco, Frontera. Distr. - same. Types o USNM. Ref. - Hull, 1949d:269, fig. 6a (head).

\section{Genus Palpada Macquart}

PALPADA Macquart, 1834:512. Type-species, scutellata Macquart (mon.) = scutellaris (Fabricius).

Doliosyrphus Bigot, 1882a:cxx. Type-species, scutellatus Bigot (Williston, $1887 \mathrm{~b}: 178$ ) = scutellaris (Fabricius).

Dolyosyrphus, Bigot, 1883b:228, misspelling.

Ref. - Curran, 1930c, 1934b:407 (key, as part of Eritalis).

AEMULA (Williston), 1891:64, pl. 2:5 (habitus) (Eristalis). Type-locality: Mexico, Guerrero: Río Papagaio, $1200 \mathrm{ft}$.; Chilpancingo, $4600 \mathrm{ft}$.; Rincón, $2800 \mathrm{ft}$.; Venta de Zopilote $2800 \mathrm{ft}$.; Guatemala, San Geronimo; Panama: Bugaba. Distr. - Mexico (Guerrero), Gua- 
temala, Panama, Guyana, Colombia. Types o $q \mathrm{BM}(\mathrm{NH})$. Ref. - Hull, 1925b:288 (redescription), pl. 1:1 (abdomen), 2:18 (hind leg). N. COMB.

AGRORUM (Fabricius), 1787:335 (Syrphus). Type-locality: "America". Distr. - U.S.A. (Louisiana, Florida), West Indies (Cuba, Puerto Rico), Honduras, Panama, Venezuela, Peru, Brazil, Paraguay, Argentina (Tucumán, Jujuy, Formosa, Santa Fe, Entre Ríos, Córdoba). Types of o MC*. Ref. - Hull, 1925a:38 (description), pl. 1:3 (abdomen), 2:14 (mesonotum); Bean, 1949:143, fig. 4 (male genitalia). N. COMB.

cubensis Macquart, 1842:102 (42) (Eristalis). Type-locality: Cuba, Havana. Type of OXF*.

?sallei Giglio-Tos, 1892c:5 (Eristalis). Type-locality: Mexico. Type $q$ UTOR*

sallaei, various authors, misspelling.

ALbIFRONS (Wiedemann), 1830:189 (Eristalis). Type-locality: "Brazil". Distr. - U.S.A. (Louisana to North Carolina \& Florida); Cuba, Mexico, Guatemala, Panama, Colombia, Venezuela, Surinam, Brazil. Type or VMNH. Ref. - Hull, 1925a:35 (redescription), pl. 1:7 (abdomen); Bean, 1949:144, fig. 3 (male genitalia) N. COMB.

senicula Loew, 1866a:168 (1872b:42) (Eristalis). Type-locality: Cuba Type ơ MCZ.

ALBIVENTRIS (Bigot), 1880b:228 (Eristalomyia). Type-locality: Uruguay, Montevideo. Distr. - same. Type o $\mathrm{BM}(\mathrm{NH})$. N. COMB.

AMazoN (Curran), 1930c:23 (Eristalis). Type-locality: Brazil, Amazonas, Rio Caiari-Uaupés. Distr. - Venezuela, Brazil (Amazonas). Type o AMNH*. N. COMB.

ARGYROPILA (Hull), 1938:121, fig. 10 (head) (Eristalis). Type-locality: French Guiana, Mana River. Distr. - same. Type o CM. N. COMB. ATrimana (Loew), 1866a:167 (1872b:41) (Eristalis). Type-locality: "Cuba". Distr. - Cuba, Jamaica, Haiti, Puerto Rico, ?Venezuela [AMNH]. Type of MCZ. Ref. - Williston, 1887b:173 (description); Curran, 1930c:16 (redescription). N. COMB.

?willistoni Townsend, 1895a:47 (Eristalis). Type-locality: Jamaica, Bath; San Domingo. Types of $\sigma^{\pi} \mathrm{BM}(\mathrm{NH})$ \& USNM. [Williston, $1887 \mathrm{~b}: 173$, refers to this species].

BEQUAERTI (Hull), 1942a:84 (Eristalis). Type-locality: Paraguay, Villarica. Distr. - same. Type o MCZ*. N. COMB.

antiopa Hull, 1942a:87 (Eristalis). Type-locality: Paraguay, Villarica. Type of MCZ*. N. SYN.

BISTELlata (Hul1), 1935:328, figs. 1 (head), 6 (hind tibiae) (Eristalis). Type-locality: Peru, Piches \& Perené. Distr. - same. Type + USNM*. N. COMB.

BRAZILIENSIS (Goot), 1964:214 (Eristalis; nom. nov. for trifasciata Macquart). Distr. - Brazil. N. COMB.

trifasciata Macquart, 1842:99 (39), pl. 10, fig. 1 (head) (Eristalis; preocc. Say, 1830). Type-locality: "Brazil". Type ? MNHN? (not found - JRV).

BREVIVILloSA (Hull), 1937a:173, figs. 2 (abdomen), 4 (hind leg) (Eristalis). Type-locality: Peru, Lima. Distr. - same. Type \& USNM*. N. COMB

Caliginosa (Walker), 1849:621 (Eristalis). Type-locality: ?. Distr. - ? (probably Neotropical). Type \& BM(NH)*. N. COMB. 
?CALOMERA (Bigot), 1880b:229 (Eristalomyia?). Type-locality: "Amer. merid.". Distr. - same. Type o $\mathrm{BM}(\mathrm{NH})$. N. COMB.

?CALOPS (Bigot), 1880b:227 (Eristalomyia). Type-locality: "Colombia". Distr. - same. Type o $\mathrm{BM}(\mathrm{NH})$.

CHIlena (Rondani), 1863:5 (Eristalomyia). Type-locality: "Chile". Distr. - Mexico?, Jamaica?, Guyana?, Brazil?, Chile (south to Aysén), Argentina (doubtful records all from Fluke, 1957:137). Type o MIZUN. N. COMB.

lateralis Walker, 1837:347 (Eristalis; preocc. Fallén, 1817). Typelocality: "Chile". Type o BM(NH)*.

porteri Curran, 1935:8 (Eristalis). Type-locality: Chile, "probably Valparaíso". Type $0^{*} \mathrm{AMNH}^{*}$. N. SYN.

CHILENSIS (Philippi), 1865:743 (Eristalis). Type-locality: Chile, Santiago. Distr. - same. Type ? MNHNS. N. COMB.

CLARIPENNIS (Hull), 1949g:123 (Eristalis). Type-locality: Brazil, Santa Catarina, Nova Teutônia. Distr. - Peru, Brazil (Santa Catarina). Type ơ Hull. N. COMB.

Clarissima (Giglio-Tos), 1892c:5 (Eristalis). Type-locality: Mexico, Tuxpango. Distr. - same. Types o UTOR*. N. COMB.

Claudia (Curran), 1930c:18 (Eristalis). Type-locality: Panama, Canal Zone, Barro Colorado Island. Distr. - same. Type o AMNH*. N. COMB.

?COLUMBicA (Macquart), 1855:108 (88) (Eristalis). Type-locality: Colombia. Distr. - same. Type of BM(NH). N. COMB.

CONCOLOR (Philippi), 1865:743 (Eristatis). Type-locality: Chile, Valparaíso. Distr. - same. Type ? MNHNS. N. COMB.

CONGRUA (Wulp), 1888:371 (Eristalis). Type-locality: Argentina, Prov. Tucumán. Distr. - same. Type $\sigma^{*}$ ML? (lost? - not found - JRV). N. COMB.

CoNICA (Fabricius), 1805:190 (Milesia). Type-locality: "America meridionali". Distr. - Guatemala, Colombia, Guyana, Brazil (Rio de Janeiro, Mato Grosso). Type o* o MC*. Ref. - Hull, 1925b:290 (redescription), pl. 1:6 (abdomen). N. COMB.

CORA (Hull), 1949g:121 (Eristalis). Type-locality: Brazil, Santa Catarina, Nova Teutônia. Distr. - same. Type ơ Hull. N. COMB.

CoSmia (Schiner), 1868:362 (Eristalis). Type-locality: "Venezuela"). Distr. - Mexico (Veracruz), Ecuador, Colombia, Peru, Argentina (Tucumán). Types $\sigma^{*}+\mathrm{VMNH}^{*}$. N. COMB.

CROCEIMACULATA (Jacobs), 1900:107 (Eristalis). Type-locality: Argentina, Tierra del Fuego, Isla de los Estados, "Golfe Saint-Jean". Distr. - same. Type o ?

?CROCEIPES (Bigot), 1880b:227 (Eristalomyia). Type-locality: "Amer. merid.". Distr. - same. Type \& $\mathrm{BM}(\mathrm{NH})$. N. COMB.

DECORA (Perty), 1830:185, tab. 37, fig. 3 (habitus) (Eristalis). Type-locality: Brazil, "Provincia Piauhiensis". Distr. - same. Type(s) ? ZSM. N. COMB.

DIABLLIS (Hull), 1935:330, figs. 3 (scutellum), 11 (hind leg) (Eristalis). Type-locality: Mexico, Mexico City. Distr. - Mexico. Type o USNM*. N. COMB.

Diminuta (Walker), 1849:622 (Eristalis). Type-locality: "Mexico". Distr. - same. Type o $\mathrm{BM}(\mathrm{NH})$. N. COMB.

UISTINGUENDA (Wiedemann), 1830:191 (Eristalis). Type-locality: Uruguay, Montevideo. Distr. - Paraguay, Uruguay, Argentina. Type o VMNH*. Ref. - Curran, 1930c:8 (descriptive note). N. COMB. 
xanthaspis Wiedemann, 1830:191 (Eristalis). Type-locality: ? (not given). Type \& VMNH? (not found - JRV).

DoRIS (Curran), 1930c:20 (Eristalis). Type-locality: Brazil, Mato Grosso, Chapada. Distr. - Brazil (Mato Grosso, Rio de Janeiro). Type o AMNH*. N. COMB.

DOROTHEA (Hull), 1942a:88 (Eristalis). Type-locality: Paraguay, Villarica. Distr. - same. Type o $\mathrm{MCZ}^{*}$. N. COMB.

ELEGANS (Blanchard), 1852:406 (Eristalis). Type-locality: Chile, Coquimbo. Distr. - Chile (Santiago — rauco), Argentina (Saeta, Neuquén). Type(s) ? MNHN? (not found - JRV). Ref. - Hull, 1925a:26 (description), pl.2:13 (abdomen), 16 (mesonotum). N. COMB.

phitippii Schiner, 1868:363 (Eristalis). Type-locality: "Chile". Types 우 VMNH*.

ERRATICA (Curran), 1930c:19 (Eristalis). Type-locality: Panama, Canal Zone, Barro Colorado Island. Distr. - Panama, Colombia, Venezuela, Guyana, Trinidad, Ecuador, Peru, Brazil. Type $0^{*} \mathrm{AMNH}^{*}$. N. COMB.

EXPICTA (Walker), 1860:290 (Eristalis). Type-locality: "Mexico". Distr. same. Type o $\mathrm{BM}(\mathrm{NH}) *$ N. COMB.

FAMILIARIS (Walker), 1860:290 (Eristalis). Type-locality: "Mexico". Distr. - same. Type o $\mathrm{BM}(\mathrm{NH}) *$. N. COMB.

FASCIATA (Wiedemann), 1819:51 (Eristalis). Type-locality: "Brazil". Distr. - Mexico, Guatemala, Panama, Venezuela, Guyana, Trinidad, Ecuador, Brazil. Types o* of VMNH* \& ZMB. Ref. - Hull, 1925a:34 (description), pl. 1:6 (abdomen), 2:15 (mesonotum). N. COMB. podagra Macquart, 1842:98 (38) (Eristalis). Type-locality: Brazil, Pará. Type \& MNHN*.

proxima Rondani, 1848:69 (Eristalis). Type-locality: "Brazil". Type ? ?

bifasciata Macquart, 1855:107 (87) (Eristalis). Type-locality: "Brazil". Types o OXF*.

?surinamensis Macquart, 1842:113 (53) (Eristalis; preocc. Degeer, 1776). Type-locality: "Surinam". Types o MNHN*. [probably not fasciata].

Fasciculata (Curran), 1938:2 (Eristalis). Type-locality: Brazil, São Paulo. Distr. - Brazil, Argentina (Tucumán, Neuquén). Type ơ AMNH*. N. COMB.

FLAVIPENNIS (Macquart), 1842:112 (52) (Eristalis). Type-locality: "Guyane". Distr. - same, Type \& MNHN*. N. COMB.

Flavoscutellate (Hull), 1937a:173, figs. 1 (tip of abdomen), 7 (hind leg) (Eristalis). Type-locality: Costa Rica, Hiquito, San Mateo. Distr. - same, Type of USNM*. N. COMB.

FLOREA (Hull), 1925a:39, pl, 1:3 (abdomen) (Eristalis). Type-locality: Guyana, Arakaba \& Bartica, Kartabo. Distr. - Mexico (Tabasco), Panama, Guyana, Trinidad. Type on OhSU. N. COMB.

FUliginosa (Hull), 1935:327, figs. 4 (scutellum), 9 (head), 10, 12 (hind femur) (Eristalis). Type-locality: Costa Rica, Atenas. Distr. same. Type o USNM*. N. COMB.

FUlviPES (Bigot), 1880z:225 (Eristalomyia). Type-locality: "Mexico". Distr. - same. Type o BM(NH). N. COMB.

FULNITARSIS (Rondani), 1863:6 (Eristalomyia; nom. nov. for rufitarsis Macquart, 1855). Distr. - Colombia. N. COMB.

rufitarsis Macquart, 1855:109 (89) (Eristalis; preocc. Macquart, 1842). Type-locality: "Colombia". Type o BM(NH). 
FUNEREA (Rondani), 1850b:358 (Eristalis). Type-locality: "America aequatoriali" [Ecuador, Rio Napo]. Distr. - same. Type o MIZUN. N. COMB.

funescens, Williston, 1886:219, misspelling.

FURCATA (Wiedemann), 1819:51 (Eristalis). Type-locality "Brazil". Distr. - Mexico (Durango), south to Peru and Argentina Type $\sigma$ VMNH*. Ref. - Hull, 1925a:29 (description), pl. 2:11 (abdomen), 20 (mesonotum). N. COMB.

femorata Macquart, 1842:100 (40), pl. 3, fig. 6 (head) (Eristalis). Type-locality: Brazil, Rio de Janeiro. Type of MNHN*.

tenebrica Walker, 1849:601 (Merodon). Type-locality: ? Type क $\mathrm{BM}(\mathrm{NH})$.

florida Walker, 1852:245 (Eristalis). Type-locality: "Brazil". Type o $\mathrm{BM}(\mathrm{NH})$

FUSCIPENNIS (Macquart), 1846:256 (128), pl. 11, fig. 5 (wing) (Eristatis). Type-locality: "Surinam". 'Distr. - same. Type 'o MRHNB. N. COMB.

GENICUlata (Fabricius), 1805:237 (Eristalis). Type-locality: "America meridionali". Distr. - Surinam, Brazil. Type o MC*. N. COMB.

fumipennis Stephens, 1846:29, pl. 47:3 (habitus) (Eristalis). Typelocality: unknown. Type o $\mathrm{BM}(\mathrm{NH})$. Ref. Verrall, 1901:672. N. SYN.

GENICULOSA Thompson (nom. nov. for geniculata Bigot). Distr. - Argentina (Buenos Aires). NOM, NOV.

geniculata Bigot, 1883a:cxx (Doliosyrphus; preocc. Fabricius, 1805). Type-locality: Argentina, Buenos Aires. Types o o $\mathrm{BM}(\mathrm{NH})$.

GLABELla (Hull), 1942a:85 (Eristalis). Type-locality: Panama, Canal Zone, Summit. Distr. - same. Type o MCZ*. N. COMB.

GUNDLACHI (Loew), 1866a:166 (1872b:40) (Eristalis). Type-locality: "Cuba". Distr. - same. Types ơ 을. $\mathrm{MCZ}$. N. COMB.

GUYANENSIS (Goot), 1964:213 (Eristalis; nom. nov. for pygmaea Macquart). Distr. - Surinam. N. COMB.

pygmaea Macquart, 1842:114 (54) (Eristalis; preocc. Zetterstedt, 1838). Type-locality: "Surinam". Type o MNHN*.

HORTORUM (Fabricius), 1775:764 (Syrphus). Type-locality: "America". Distr. - West Indies, Cuba, Puerto Rico, St. Thomas. Type ? Kiel? (not found - JRV). Ref. - Williston, 1887b:173 (redescription), pl. 7:10 (wing); Hull, 1925a:41 (description), pl. 1:8 (abdomen). N. COMB.

INCA (Bigot), 1880b:217 (Eristalis). Type-locality: "Peru". Distr. — same. Type $\sigma \mathrm{BM}(\mathrm{NH})$. N. COMB.

INCERTA (Bigot), 1880b:220 (Eristalomyia). Type-locality: "Brazil". Distr. - same. Type \& OXF. N. COMB.

Incubus (Hull), 1943f:29 (Eristalis). Type-locality: Brazil, Paraná, Curitiba. Distr. - same. Type o Hull. N. COMB.

INTERRUPTA (Fabricius), 1805:239 (Eristalis). Type-locality: "America meridionali". Distr. - same. Type o MC*. N. COMB.

INVERSA (Wiedemann), 1830:161 (Eristalis). Type-locality: Surinam.

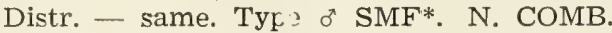

LANGI (Curran), 1934b:411 (Eristalis). Type-locality: Guyana, Kamakusa. Distr. - Guyana, Peru, Brazil (Mato Grosso). Type of AMNH*. N. COMB.

MACUla (Sack), 1941:112 (1951:108) (Eristalis). Type-locality: Peru, Pachitea River. Distr. - Ecuador, Colombia, Peru. Types $\sigma^{*}+\mathrm{MD}$. N. COMB. 
stigmatica Hull, 1944d:45 (Eristalis). Type-locality: Colombia, Atrato Valley. Type o $\mathrm{BM}(\mathrm{NH}) *$. N. COMB.

MEIGENII (Wiedemann), 1830:177 (Eristalis). Type-locality: Uruguay, Montevideo. Distr. - Uruguay, Argentina (Buenos Aires), Chile (Santiago - Malleco). Type o" VMNH*. Ref. - Hull, 1925a:29 (redescription), figs. 12 (abdomen), 18 (mesonotum), 22 (head). N. COMB.

quadraticornis Macquart, 1842:111 (51), pl, 10, fig. 2 (head) (Eristalis). Type-locality: "Chile". Type o MNHN*.

testaceiscutellata Macquart, 1850:442 (138) (Eristalis). Type-locality: "Chile". Types o" MNHN*.

Joveifrons Thomson, 1869:491 (Eristalis). Type-locality: Argentina, Buenos Aires. Type of NRS*.

MELANASPIS (Wiedemann), 1830:176 (Eristalis). Type-locality: "Brazil". Distr. - Brazil, Bolivia, Paraguay.' Types o VMNH \& ZMB*. Ref. - Hull, 1925b:287 (redescription). N. COMB.

nigriscutellata Macquart, 1848:201 (41) (Eristalis). Type-locality: "Brazil". Type ơ MNHN*.

nigroscutellata, Williston, 1886:318, Fluke, 1957:138, misspelling.

volatica Williston, 1888:280 (Eristalis). Type-locality: Brazil, Mato Grosso, Chapada; Corumbá; Rio de Janeiro. Types $0^{*}$ AMNH*.

MEXICANA (Macquart), 1847:75 (59). (Eristalis). Type-locality: "Mexico". Distr. - same. Type $0^{*} \mathrm{BM}(\mathrm{NH})$. N. COMB.

MINUTALIS (Williston), 1891:64, pl. 2:6 (habitus), 6a (head) (Eristalis). Type-locality: Mexico, Tabasco, Teapa. Distr. - Mexico (Tabasco), Honduras, Guatemala, Guyana, Colombia, Brazil. Types $0^{*} q$ $\mathrm{BM}(\mathrm{NH})$. Ref. - Hull, 1925a: 42 (redescription). N. COMB.

Mirabilis (Hull), 1925b:305, pl. 2:11 (mesonotum) (Eristalis). Typelocality: Colombia, Vista Nieve. Distr. - Colombia. Type q AMNH*. N. COMB.

MITIS (Curran), 1930c:11 (Eristalis). Type-locality: Panama, Canal Zone, Corozal. Distr. - Mexico (Veracruz), Panama. Type of AMNH*. N. COMB.

MONTICOLA (Röder), 1892:9, fig. 1 (habitus) (Eristalis; nom. nov. for montana Röder). Distr. - Colombia. N. COMB.

montana Röder, 1886:263 (Eristalis; preocc. Williston, 1882). Typelocality: Colombia, Cordilleren, Páramo, $3600 \mathrm{~m}$. Types ? (Halle).

NIGRIPENNIS (Macquart), 1855:108 (88) (Eristalis). Type-locality: "Colombia". Distr. - same. Type o OXF. N. COMB.

NIGRIPES (Wiedemann), 1830:165 (Eristalis). Type-locality: "Brazil". Distr. - Surinam, Brazil, Argentina. Type o VMNH*, N. COMB.

obligua (Curran), 1940:14 (Eristalis). Type-locality: Guyana, Mazaruni. Distr. - Guyana, Peru. Type o AMNH*. N. COMB.

OBSOLETA (Wiedemann), 1830:175 (Eristális). Type-locality: "Brazil". Distr. - Guatemala, Venezuela, Brazil. Types o $q$ VMNH. Ref. Hull, 1925b:306 (description); Bean, 1949:146, fig. 9 (male genitalia). N. COMB.

?pachypoda Bigot, 1880b:224 (Eristalomyia). Type-locality: "Mexico". Type of $\mathrm{BM}(\mathrm{NH})$.

ochracea (Williston), 1888:279 (Eristalis). Type-locality: Brazil, Mato Grosso, Chapada. Distr. - Mexico (Tajasco), Colombia, Guyana, Brazil (São Paulo, Mato Grosso). Type ơ AMNH*. N. COMB.

alacris Curran, 1934b:413 (Eristalis). Type-locality: Guyana, Kamakusa. Type o AMNH*. 
ORNATA (Townsend), 1897a:21 (Eristalis). Type-locality: Mexico, Veracruz, Río Nautla, San Rafael. Distr. - U.S.A. (California), Mexico (Veracruz). Tpes o $\mathrm{BM}(\mathrm{NH})$. N. COMB.

PARVUla (Williston), 1888:282 (Eristalis). Type-locality: Brazil, Mato Grosso, Chapada. Distr. - same. Type o AMNH*. N. COMB.

PENALTis (Curran), 1934b:412 (Eristalis). Type-locality: Guyana, Kartabo. Distr. - same. Type o AMNH*. N. COMB.

PRECIPUA (Williston), 1888:280 (Eristalis). Type-locality: Brazil, Swo Paulo; Mato Grosso, Chapada. Distr. - Brazil (São Paulo, Mato Grosso, Santa Catarina); Argentina (Formosa). Types o o AMNH*. Ref. - Hull, 1925b:286 (description), pl. 1:8 (mesonotum). N. COMB.

PUSILLA (Macquart), 1842:114 (54) (Eristalis). Type-locality: French Guiana, Mana River. Distr. - U.S.A. (Texas), Mexico, Cuba, Guatemala, Ecuador, Colombia, Argentina (Tucumán, Formosa). Type o MNHN*. Ref. - Hull, 1925a:36 (description), pl. 1:2 (abdomen). 2:17 (mesonotum); Bean, 1949:144, fig. 2 (male genitalia). N. COMB.

annulipes Macquart, 1842:109 (49) (Eristalis). Type-locality: Brazil, Rio de Janeiro. Types of o MNHN*. N. SYN.

tricolor Jaennicke, 1867:400 (Eristalis; preocc. Meigen, 1803). Typelocality: "Mexico". Type \& SMF.

PUsio (Wiedemann), 1830:192 (Eristalis). Type-locality: "Brazil". Distr. Puerto Rico, Ecuador, Brazil, Argentina. Type \& VMNH*. Ref. Curran, 1930c:13 (redescription). N. COMB.

PUSIordes (Hull), 1951b:68 (Eristalis). Type-locality: Peru, Chanchamayo. Distr. - same. Type ơ Hull. N. COMB.

Pygolampa (Wiedemann), 1830:161 (Eristalis). Type-locality: "Brazil". Distr. - Guyana, Brazil, Paraguay, Argentina (Tucumán). Types $o^{*}$ VMNH \& MC*. Ref. - Hull, 1925b:304 (redescription). N. COMB.

QUITENSIS (Macquart), 1855:109 (89) (Eristalis). Type-locality: Ecuador, Quito. Distr. - same. Type o $\mathrm{BM}(\mathrm{NH}) *$. N. COMB.

RUFICEPS (Macquart), 1842:111 (51) (Eristalis). Type-locality: Colombia, Santa Fe de Bogotá. Distr. - Mexico, Colombia, Ecuador, Venezuela, Bolivia. Type o MNHN*. Ref. - .Hull, 1925b:307 (description), pl. 2:10 (head). N. COMB.

thoracica Jaennicke, 1867:399 (Eristalis). Type-locality: “Mexico". Types of o SMF.

RUFIPEDES Thompson (nom. nov. for rufipes Macquart). Distr. - Brazil. NOM. NOV.

rufipes Macquart, 1842:102 (42) (Eristalis; preocc. Fabricius, 1805). Type-locality: Brazil, "parte meridionale des Campos Geraes". Type o MNHN*.

RUfIVENTRIS (Macquart), 1846:257 (129) (Eristalis). Type-locality: "Colombia". Distr. - U.S.A. (Texas), Mexico, Guatemala, Honduras, Colombia, Ecuador, Peru, Brazil, Argentina (Formosa, Tucumán). Type ơ MNHN? (not found - JRV). Ref. - Hull, 1925a:33 (description), pl. 1:4 (abdomen). N. COMB.

praeclarus Giglio-Tos, 1892c:5 (Eristalis). Type-locality: Mexico, Tuxpango. Type o UTOR*.

RUfoscutellata (Sack), 1921:142, fig. 16 (head) (Eristalis). Type-locality: Paraguay, St. Trinidad. Distr. - Brazil (São Paulo, Santa Cata- 
rina, Paraná), Paraguay, Argentina (Tucumán). Types ơ $q$ DEI \& SMF*. N. COMB.

cyatheus Hull, 1925b:287, pl. 1:9 (mesonotum), 2:13 (head) (Eristalis). Type-locality: Brazil, São Paulo, São. Sebastião. Type + AMNH*. N. SYN.

SCHISTACEA (Williston), 1888:282 (Eristalis). Type-locality: Brazil, Mato Grosso, Chapada. Distr. - same. Type o AMNH (lost - FCT). N. COMB.

Scopanthus (Hull), 1944j:45 (Eristalis). Type-locality: Peru, Lima. Distr. - same. Type o CU. N. COMB.

SCUTELLARIS (Fabricius), 1805:190 (Milesia). Type-locality: "America meridionali". Distr. - U.S.A. (New Mexico), south to Brazil. Type .+ MC*. Ref. - Hull, 1925b:291 (description), pl. 2:16, 19 (head); Doesburg, 1963: 23, fig. 1 (habitus), 1a (head), 1b (hind leg), 2, 4 (puparium), 3 (habitus). N. COMB.

scutellata Macquart, 1834:513, pl. 11, fig. 19 (mouthparts). Typelocality: "Brazil". Types of o MNHN*. N. SYN.

?angustata Rondani, 1848:70 (Eristalis). Type-locality: "Brazil". Type o MIZUN.

?cognata Rondani, 1848:69 (Eristalis; preocc. Wiedemann, 1824). Type-locality: "Brazil". Type or MIZUN.

cyaneifera 'Walker, 1849:621 (Eristalis). Type-locality: ? Type ? $\mathrm{BM}(\mathrm{NH})$.

limbatinevris Macquart, 1850:441 (137) (Eristalis). Type-locality: "Brazil". Types o OXF*. N. SYN.

?fascithorax Macquart, 1850:443 (139) (Eristalis). Type-locality: "Amérique". Types o" o MNHN*.

?agnata Rondani, 1850b:358 (Eristaliss nom. nov. for cognata Rondani).

?scutellata Bigot, 1883a:cxx (Doliosyrphus; preocc. Macquart, 1834). Type-locality: "Panama". Types on \& BM(NH).

bimaculata Bigot, 1883b:222 (Priomerus). Nomen nudum.

rileyi Williston, 1887b:178 pl. 8:8 (head) (Doliosyrphus). Type-locality: U.S.A., New Mexico. Types ơ USNM.

SEMICIRCulus (Walker), 1852:249 (Eristalis). Type-locality: "Honduras". Distr. - same. Type o BM(NH). IT. COMB.

SOLENNIS (Walker), 1852:245 (Eristalis). Type-locality: ? Distr. - Mexico (Yucatán), Brazil, Argentina (Formosa, Tucumán, Río Negro). Type ơ BM(NH)*. N. COMB.

agrorum, Hull, 1925b:289, pl. 1:3 (abdomen), misidentification.

mus Curran, 1930c:21 (Eristalis). Type-locality: Brazil, Mato Grosso, Corumbá. Type o AMNH*. N. SYN.

SPECTABILIS (Hull), 1925b:289, pl. 1, fig. 2 (abdomen) (Eristalis). Typelocality: Venezuela, Caura Valley. Distr. - Venezuela, Guyana, Peru. Type \& MCZ*. N. COMB.

TAENIA (Wiedemann), 1830:174 (Eristalis). Type-locality: Uruguay, Montevideo. Distr. - Colombia, Venezuela, Brazil (São Paulo, Rio de Janeiro), Uruguay, Argentina (Formosa, Tucumán, Santa Fe, Córdoba). Types VMNH* \& ZMB. Ref. - Hull, 1925a:38 (description); Curran, 1930c:13 (descriptive notes). N. COMB.

TATEI (Curran), 1930c:9 (Eristalis). Type-locality: Venezuela, Mt. Duida. Distr. - same. Type o AMNH*. N. COMB.

TESTACEICORNIS (Macquart), 1850:442 (138) (Eristulis). Type-locality: "Mexico". Distr. - U.S.A. (California, Arizona), Mexico, Panama, 
Colombia, Venezuela, Trinidad, Ecuador, Peru. Types $q$ OXF $^{*}$. N. COMB.

sackenis Bigot, 1880b:224 (Eristalis). Type-locality: "Mexico". Type q $\mathrm{BM}(\mathrm{NH}) *$ * N. SYN.

sumischrasti Giglio-Tos, 1892d:1 (Eristalis). Type-locality: Mexico, Tehuacán. Type ơ UTOR*. N. SYN.

sumichrasti, Giglio-Tos, 1893:6, Aldrich, 1905:389, Kertész, 1910:237, Fluke, 1957:143, emendation.

THalia (Hull), 1942a:86 (Eristalis). Type-locality: Colombia, Dept. Cundinamarca, Bogotá, $2600 \mathrm{~m}$. Distr. - same. Type o MCZ*. N. COMB.

TRIANgUlaris (Giglio-Tos), 1892c: (Eristalis). Type-locality: Mexico, Cuautla. Distr. - U.S.A. (California), Mexico, Cuba, Trinidad, Guatemala, Brazil. Type \& UTOR*. Ref. - Hull, 1925a:35 (description), pl. 2:19 (mesonotum). N. COMB.

TRIGONA (Williston), 1891:61, pl. 2:4 (habitus), 4a (head) (Eristalis). Type-locality: Mexico, Guerrero, Xucumanatlán, 7000 ft.; Omiltemi, $8000 \mathrm{ft}$. Distr. - Mexico (Durango, Guerrero). Types o o $\mathrm{BM}(\mathrm{NH})$. N. COMB.

atropos Giglio-Tos, 1892d:1 (Eristalis). Type-locality: "Mexico". Type $0^{\top}$ UTOR*. N. SYN.

URotAENIA (Curran), 1930c:15 (Eristalis). Type-locality: Brazil, Mato Grosso, Chapada. Distr. - Surinam, Ecuador, Peru, Brazil (Mato Grosso). Type o AMNH*. N. COMB.

VERA (Hull), 1949g:120 (Eristalis). Type-locality: Brazil, Santa Catarina, Nova Teutônia. Distr. - same. Type ơ Hull. N. COMB.

VIERECKI (Curran), 1930c:10 (Eristalis). Type-locality: Colombia, Vista Nieve. Distr. - Colombia, Bolivia. Type o AMNH. N. COMB.

VINETORUM (Fabricius), 1798:562 (Syrphus; as vinctorum, emended by Fabricius, 1799:48). Type-locality: "America Insulis". Distr. U.S.A. (Wisconsin to Pennsylvania), south to Brazil and Argentina (Misiones, Formosa); Galápagos, Cuba, Haiti, Puerto Rico, Virgin Islands, Trinidad. Type Kiel (lost, Zimsen, 1964:478). Ref. - Williston, 1887b:171 (redescription), pl. 7:8a (hind leg); Hull, 1925a:40 (description), pl. 1:9 (abdomen); Bean, 1949:146, fig. 8 (male genitalia). N. COMB.

?surinamensis Degıer, 1776:145, pl. 29, fig. 1. (habitus) (Musca). Type-locality: "Surinam". Type ? NRS [lost, Persson, in litt.]. trifasciata Say, 1830:165 (Eristalis). Type-locality: "Mexico". Type ? lost.

uvarum Walker, 1849:623 (Eristalis). Type-locality: "Jamaica". Types o $\mathrm{BM}(\mathrm{NH})$.

soulouguensis Bigot, 1880b:228 (Eristalis). Type-locality: "Haiti". Type o $\mathrm{BM}(\mathrm{NH})$.

hirtipes Bigot, 1883a:cxxi (Doliosyrphus). Type-locality: Panama. Type of $\mathrm{BM}(\mathrm{NH}) *$. N. SYN.

tritimbata Giglio-Tos, 1892c:5 (Eristalis). Type-locality: Mexico, Tampico. Type o UTOR*. N. SYN.

\section{Genus Meromacrus Rondani}

Plagiocera Macquart, 1842:119 (59) (preocc. Klug. 1834). Type-species, Milesia cruciger Wiedemann (orig. des.) = acutus (Fabricius). 
Meromacrus Rondani, 1848:70. Type-species, ghilianii Rondani (mon.).

Pteroptila Loew, 1866a:165 (1872b:39). Type-species, decorus Loew (Coquillett, 1910a:598).

Promilesia Lynch Arribálzaga, 1892a:241 (1892c:97). Type-species, nectarinoides Lynch Arribálzaga (mon.). N. SYN.

Metameromacrus Hull, 1942e:1. no type-species designated. Nomen nudum. Thalamopales Hull, 1949d:401. Type-species, Helophilus scitus Walker (orig des.).

Refs. - Sack, 1920 (rev., key); Hull, 1942e (rev., key).

ABdominalis Sack, 1920:261, fig. y (mesonotum). Type-locality: Peru . Distr. - same. Type o MD.

ACUTus (Fabricius), 1805:189 (Milesia). Type-locality: U.S.A., "Carolina". Distr. - U.S.A. (Virginia to Florida \& Texas), Mexico, Guatemala, British Honduras, Cuba. Types ? lost [Zimsen, 1964:473]. Ref. - Williston, 1887b:180 (redescription), pl. 8:1 (wing), 1a (abdominal tip), 1b (hind leg), 1c (head); Sack, 1920:260 (description); fig. c' (mesonotum); Hull, 1942e:11, figs. 9 \& 10 antenna).

cruciger Wiedemann, 1830:105 (Milesia). Type-locality: Georgia. Types of $\mathrm{VMNH}$ ?

crucifer, Bigot, 1857b:337, misspelling.

millesiformis Macquart, 1834:500 (Mallota). Type-locality: Cuba. Type ơ (Lille?).

milesiformis, Fluke, 1957:147, misspelling.

maculatus Macquart, 1850:451 (147), pl. 14, fig. 2 (wing) (Milesia). Type-locality: "Afrique" [error?] Type o MNHN.

AEmulus (Williston), 1888:283 (Pteroptila). Type-locality: Brāzil, Mato Grosso, Chapada. Distr. - same. Types ơ AMNH*. Ref. - Sack, 1920:260 (description), figs. w (wing), x (mesonotum).

ANNA Curran, 1936a:4. Type-locality: Panama, Canal Zone, Fort Randolph. Distr. - Panama, Guyana, Peru, Brazil. Type o* AMNH*.

AURIFER Hull, 1942e:4, fig. 12 (mesonotum). Type-locality: Brazil, Santa Catarina, Nova Teutônia. Distr. $\longrightarrow$ same. Type o AMNH**

BASIGER (Walker), 1860:290 (Eristalis). Type-locality: "Amazon Region". Distr. - Brazil, Argentina (Tucumán). Type q BM(NH). Ref. Sack, 1920:270 (description), figs. d' (head), f' (mesonotum).

milesioides Bigot, 1880b:226 (Eristalomyia). Type-locality: "Brazil". Type o $\mathrm{BM}(\mathrm{NH})$.

BRUNerI Curran, 1936a:2. Type-locality: "Cuba". Distr. - same. Type $q$ AMNH*.

BRUNNEUS Hull, 1942e:9, fig. 8 (antenna). Type-locality: Guyana, Wismar. Distr. - same. Type of AMNH*.

CANusium (Walker), 1849:566 (Milesia). Type-locality: "Cape of Good Hope" (error, Hull, 1942e:9). Distr. - Mexico (Nayarit). Type \&

BM(NH). Ref. - Hull, 1942e:9 (description), fig. 1 (mesonotum).

CERES Hull, 1942b:11. Type-locality: "Brazil". Distr. - same. Type o Hull. CINCTUS (Drury), 1770:109, pl. 45, fig. 6; (index to vol. 1, p. [2]; 1773) (Musca). Type-locality: "Jamaica". Distr. - West Indies. Type ? ? Ref. - Williston, 1887b:182 (redescription); Sack, 1920:265 (description).

pinguis Fabricius, 1775:763 (Syrphus). Type-locality: "America" Type ? ? [Zimsen, 1964:478].

ania Walker, 1849:564 (Milesia). Type-locality: "Jamaica". Type + $\mathrm{BM}$ (NH). 
CIRCUMdatus (Bigot), 1875:471 (Sphixea). Type-locality: "Colombia". Distr. - Peru, Colombia. Type of OXF. Ref. - Sack, 1920:263 (description), fig. b' (mesonotum).

Currani Hull, 1942e:5, fig. 7 (antenna). Type-locality: "British Honduras". Distr. - same. Type of AMNH*.

DECORUS (Loew), 1866a:165 (1872b:39) (Pteroptila). Type-locality: "Cuba". Distr. - same. Types o ${ }^{*}$ i MCZ. Ref. - Williston, 1887b:181 (redescription); Sack, 1920:270 (description).

DRACO Hull, 1942e:6. Type-locality: Guatemala, Quirigua. Distr. - U.S.A. (Arizona, Texas); Guatemala, British Honduras, Costa Rica. Type $0^{\pi}$ USNM.

Flavolinea Hull, 1949g:131. Type-locality: Peru, Puccalpa. Distr. -- same. Type ơ Hull.

FLUKeI Curran, 1936a:3. Type-locality: West Indies, St. Vincent. Distr. Virgin Islands. Type o AMNH*.

Fucatus Hull, 1930:143. Type-locality: Surinam, Paramaribo. Distr. same. Type \& ANSP. Ref. - Hull, 1942e:11, fig. 11 (mesonotum)

GHILIANII Rondani, 1848:71, figs. 3-5 (head), 4 (antenna). Type-locality: "Brazil". Distr. — same. Type o MIZUN.

HINEI Hull, 1942e:4. Type-locality: Peru, Middle Rio Marañón. Distr. same. Type o $\mathrm{AMNH}^{*}$.

LACONICUS (Walker), 1852:227 (Milesia). Type-locality: "Brazil". Distr. same. Type क BM(NH), N. COMB.

LINEASCRIPTA Hull, 1937a:170, fig. 10 (mesonotum \& abdomen). Type-locality: Bolivia, Beni, Ivon. Distr. — same. Type ot USNM.

Matilda Hull, 1949g:127. Type-locality: Peru, Pucallpa. Distr. - same. Type \& Hull.

Melmoth Hull, 1937b:12. Type-locality: Bolivia, Prov. of Sara. Distr. same. Type o $\mathrm{MCZ}$.

Milesia Hull, 1942e:2. Type-locality: "French Guiana". Distr. - same. Type ? Hull?

NECTARINOIDES (Lynch Arribálzaga), 1892a:243 (1892c:99), fig. 10 (head) (Promilesia). Type-locality: Argentina, Chaco; Misiones, Santa Ana \& Mocona. Distr. - Brazil (Santa Catarina); Paraguay, Argentina (Tucumán, Buenos Aires, Chaco, Misiones). Types ? MACN. N. COMB.

cingulatus Sack, 1920:259. Type-locality: Brazil, Rio Grande do Sul, Santa Cruz. Types of o HZM \& SMF*. N. SYN.

NIGER Sack, 1920:264 [also, 1921:145]. Type-locality: Bolivia, Paraguay, St. Trinidad. Distr. - Guyana, Brazil, Bolivia, Paraguay, Argentina (Tucumán, Misiones). Types MD, DEI \& SMF*.

?funereus Shannon \& Aubertin, 1933:163. Type-locality: Argentina, Misiones Terr., Posadas. Type o $\mathrm{BMI}(\mathrm{NH}) *$

oBscurus Hine, 1924:21. Type-locality: Guatemala, Los Amates. Distr. same. Type ot OhSU.

OPULENTUS (Bigot), 1883b:336 (Eristalis), Type-locality: "Cuba". Distr. same. Type o BM(NH). Ref. - Sack, 1920:267 (description); Hull, 1942e:10, figs. 2 (abdomen), 3 (mesonotum).

PACHYPUS (Wiedemann), 1830:171 (Eristalis; nom. nov. for crassipes Fabricius). Distr. - Brazil (São Paulo, Mato Grosso). Ref. - Sack, 1920:262 (descriptive note), figs. $z$ (head), a' (mesonotum); Hull, 1942c:11, fig. 6 (mesonotum). N. COMB.

crassipes Fabricius, 1805:196 (Merodon; preocc. Fabricius, 1805:237). Type-locality: "America meridionali". Type $\sigma^{*} \mathrm{MC} *$. 
simplex Schiner, 1868:365 (Plagiocera). Type-locality: "Brazil". Type 0 VMNH. N. SYN.

PLuto Hull, 1942e:7, fig. 13 (antenna). Type-locality: Peru, Middle Río Ucayali. Distr. - same. Type o AMNH*.

POTENS Curran, 1932a:8. Type-locality: Cuba, Sierra Maestra, B, de Joaquín. Distr. - same. Type o AMNH*. Ref. - Hull, 1942e:11, fig. 4 (antenna).

PRATORUM (Fabricius), 1775:765 (Syrphus). Type-locality: "America". Distr. - Panama, Guyana, Ecuador, Paraguay, Brazil, Argentina. Type ? Kiel (not found - JRV). Refs. - Sack, 1920:268 (description), fig. e' (mesonotum); Sack, 1921:143 (description), figs. 17 (mesonotum), 18a (larva), 19 (puparium), 20 (anal organ).

panamensis Curran, 1930f:11. Type-locality: Panama, Canal Zone, Barro Colorado. Type o $\mathrm{AMNH}^{*}$.

RUFICRUS (Wiedemann), 1830:105 (Milesia). Type-locality: "Cuba". Distr. - U.S.A. (Florida); Cuba. Type o ZMB. Ref. - Williston, 1887b:181 (redescription); Sack, 1920:268 (redescription); Hull, 1942e:11, fig. 5 (antenna).

SCITUS (Walker), 1857:152 (Helophitus). Type-locality: "Valley of Amazon". Distr. - same. Type o BM(NH)*. Ref. - Hull, 1949d:390, fig. 241 (head).

STRIgulus Hull, 1942e:8. Type-locality: Peru, Middle Río Ucayali. Distr. same. Type \& AMNH*.

UNICOLOR (Wulp), 1883:131, figs. 11 (habitus), 12,12 (head) (Eristalis). Type-locality: "Guadeloupe". Distr. - same. Type \& ML? [not found - JRV].

villosus Hull, 1949g:129. Type-locality: Peru, Pucallpa. Distr. - same. Type or Hull.

zonAtus (Loew), 1866a:165 (1872b:39) (Pteroptila). Type-locality: "Mexico". Distr. - same. Type o MCZ. Ref. - Williston, 1887b:182 (redescription); Sack, 1920:266 (description).

var. LoEWI (Williston), 1892:67 (Pteroptila). Type-locality: Panama, Volcan de Chiriquí. Distr. - same. Type \& $\mathrm{BM}(\mathrm{NH})$.

\author{
Tribe MILESIINI \\ Ref. - Shannon, 1926a (rev., keys)
}

Genus XуLота Meigen

Zelima Meigen, 1800:34. Type-species, Musca segris Linnaeus (Coquillett, 1910a:621). Suppressed by I.C.Z.N., 1963:339.

Heliophilus Meigen, 1803:273. Type-species, Musca sylvarum Linnaeus (mon.).

Eumeros Meigen, 1803:273. Type-species, Musca segnis Linnaeus (Coquillett, 1910a:541).

xyLota Meigen, 1822:211 (nom. nov. for Heliophilus). Type-species, Musca sylvarum Linnaeus (aut.) [In order to preserve established usage, we have followed Wirth, et al., 1965:604, in using Xylota instead of its senior synonyms, Heliophilus and Eumeros.]

ANALIS Williston, 1887b:226. Type-locality: U.S.A., California \& New Mexico. Distr. - Canada (British Columbia) to U.S.A. (Colorado), south to Mexico (Durango). Types or o USNM. 
AZUREA (Fluke), 1953a:126 (Heliophilus). Type-locality: U.S.A., New Mexico, Cloudcroft. Distr. - U.S.A. (New Mexico); Mexico (Durango). Type o UK.

BRACHYGaSter Williston, 1892:72. Type-locality: Mexico, Guerrero, Omiltemi, $8000 \mathrm{ft}$. Distr. — same. Type o $\mathrm{BM}(\mathrm{NH})$.

FLAVIFRoNS Walker, 1849:557. Type-locality: Canada, Ontario, Hudson's Bay, Albany River, St. Martin's Falls. Distr. - Alaska to Labrador, south to Canada (British Columbia) and U.S.A. (Pennsylvania); ?Mexico [Giglio-Tos, 1893:26, as communis Walker]. Types o i $\mathrm{BM}(\mathrm{NH})$.

communis Walker, 1849:557. Type-locality: Canada, Ontario, Hudson's Bay, Albany River, St. Martin's Falls. Types of o $\mathrm{BM}(\mathrm{NH})$.

NITIDULA (Fluke), 1939:368, fig. 8 (abdomen) (Heliophilus). Type-locality: Mexico, Mexico City, 10,000 ft. Distr. - same. Type on AMNH*. PIGRA (Fabricius), 1794:295 (Syrphus). Type-locality: "Germany". Distr. Holarctic; Canada (British Columbia to Quebec), south to U.S.A. (California to Florida); ?Mexico [Wirth, et. al., 1965:607]. Type ? lost (Zimsen, 1964:473).

PRETIOSA Loew, 1861:39. Type-locality: "Cuba". Distr. - same. Type o MCZ. Ref. - Williston, 1887b:237 (redescription).

STENOGASTER Williston, 1892:72. Type-locality: Mexico, Guerrero, Omiltemi, $8000 \mathrm{ft}$. Distr. - same. Types of $+\mathrm{BM}(\mathrm{NH})$.

WrLlistoNi (Goot), 1964:214 (Heliophilus; nom. nov. for rufipes Williston). Disti. - Mexico.

rufipes Williston, 1892:71 (preocc. Loew, 1873). Type-locality: Mexico, Guerrero, Omiltemi, $8000 \mathrm{ft}$. Types of $o^{\pi} \mathrm{BM}(\mathrm{NH})$.

\section{Genus Neplas Porter}

Planes Rondani, 1863:9 (preocc. Bowditch, 1825; Saussure, 1862). Typespecies, Xylota vagans Wiedemann (mon.).

NEPLAS Porter, 1927b:96 (nom. nov. for Planes Rondani). Type-species, Xylota vagans Wiedmann (aut.).

Ref. - Curran, 1941 (key).

ARIEL (Curran), 1941:302 (Planes). Type-locality: Brazil São Paulo. Distr. - same. Type o AMNH*.

ARMatipes (Curran), 1941:300 (Planes). Type-locality: Brazil, Santa Catarina, Nova Teutônia. Distr. - same. Type ơ AMNH*.

AZTECA (Curran), 1941:303 (Planes). Type-locality: Honduras, Puerto Castilla. Distr. - same. Type of AMNH*.

Bidens (Curran), 1941:300 (Planes). Type-locality: Brazil, Santa Catarina, Nova Teutônia. Distr. - Brazil (Mato Grosso, Santa Catarina). Type $\sigma^{*} \mathrm{AMNH} *$.

BOLIVIENSIS (Shannon), 1926a:44 (Planes). Type-locailty: Bolivia, Huachi, Beni. Distr. - same. - Type o USNM.

CHLORops (Hull), 1948b:7 (Planes). Type-locality: Venezuela, San Esteban. Distr. - same. Type o USNM.

CHRYSOPRESSA (Hull), 1941d:158 (Planes). Type-locality: Panama, Canal Zone, Barro Colorado Island. Distr. - same. Type o Hull.

Cuprescens (Hull), 1941d:159 (Planes). Type-locality: Panama, Canal Zone, Barı Colorado Island. Distr. - same. Type $q$ Huli.

FroNtalis (Curran), 1941:302 (Planes). Type-locality: Brazil, Mato Grosso, Chapada. Distr. - same. Type o AMNH*.

GRANDIFEMORALIS (Curran), 1934b:402, fig. 28 (hind femur) (Planes). Type-locality: Guyana, Kartabo. Distr. - same. Type o AMNH*. 
GRISEA (Hull), 1941e:281 (Plan€s). Type-locality: Brazil, São Paulo. Distr. - same. Type ơ Hull.

LYRICA (Curran), 1941:301 (Planes). Type-locality: Panama, Canal Zone, Corozal. Distr. - same. Type o AMNH*.

MINCR (Shannon), 1926a:44 (Planes), Type-locality: Bolivia, Ivón, Beni. Distr. - same. Type o USNM.

PACHYMERA (Loew), 1866a:162 (1872b:36) (Xylota). Type-locality: "Cuba". Distr. - Cuba, Puerto Rico. Type \& MCZ. Ref. - Williston 1887b:237 (redescription).

PALITARSis (Curran), 1934b:404 (Planes). Type-locality: Guayana, Kartabo. Distr. - same. Type ot AMNH*.

PANAMENA (Curran), 1941:301 (Planes). Type-locality: Panama, Canal Zone, Barro Colorado Island. Distr. - same. Type o AMNH*.

PaUxilla (Williston), 1892:71, pl. 2:9 (habitus), 9a (head), 9b (hind leg) (Xylota). Type-locality: Mexico, Guerrero, Omiltemi, $8000 \mathrm{ft}$.; Xucumanatlán, $7000 \mathrm{ft}$. Distr. - same. Types o $o^{7} \mathrm{BM}(\mathrm{NH})$.

PRoXima (Hull), 1944c:38 (Planes). Type-locality: "West Indies?", Distr. - same. Type o $\mathrm{MCZ}$.

PUMA (Curran), 1941:303 (Planes). Type-locality: Panama, Canal Zone, Barro Colorado Island. Distr. - same. Type o AMNH*.

RoNDANII (Shannon), 1926a:14 (Planes). Type-locality: Costa Rica, San Mateo, Higuito. Distr. - same. Type or USNM.

SAPPHIRINA Hull, 1951c:185. Type-locality: Peru, Chanchamayo. Distr. same. Type o Hull.

SCHILd (Shannon), 1926a:15 (Planes), Type-locality: Costa Rica, San Mateo, Higuito. Type o USNM.

SMARTI (Curran), 1941:302 (Planes). Type-locality: Guyana, Kaieteur. Distr. - same. Type o $\mathrm{BM}(\mathrm{NH}) *$

VAGABONDANS (Hull), 1941c:62 (Planes). Type-locality: Colombia, Restrepo. Distr. - same. Type o Hull.

VAGANS (Wiedemann), 1830:101 (Xylota). Type-locality: Brazil, Distr. Panama, Guyana, Brazil (Pará). Type o VMNH.

americana Schiner, 1868:367 (Syritta). Type-locality: "South America". Type o* VMNH*.

mexicana Bigot, 1884a:539 (Syritta). Type-locality: "Mexico". Type 3 $\mathrm{BM}(\mathrm{NH}) *$

VAleria (Hull), 1941e:282 (Planes). Type-locality: Brazil, São Paulo. Distr. - same. Type or Hull.

WILlistoNI (Shannon), 1926a:14 (Planes). Type-locality: Mexico, Sierra Madre, Chihuahua, about $7300 \mathrm{ft}$. Distr. - same. Type $\sigma^{\circ}$ USNM.

\section{Genus Certogaster Williston}

CERICGASTER Williston, 1888:285. Type-species, fascithorax Williston (mon.). Zonemyia Shannon, 1925a:109. Type-species, spinosa Shannon (orig. des.). ARETHIUSA Hull, 1944j:42. Type-locality: Surinam, Paramaribo, Agricultural Station 'Wolfenbuttel'. Distr. - same, Type o* CU*.

AUREOPILA Hull, 1941h:314. Type-locality: Panama, Canal Zone, Barro Colorado Island. Distr. - same. Type of Hull.

FASCITHORAX Williston, 1888:286 (as foscithorax; emendation by Kertész, 1910:308; Curran, 1930f:10). Type-locality: Brazil, Mato Grosso:

Corumbá \& Rio de Janeiro. Distr. - same. Types of o AMNH*. fuscithorax, Shannon, 1926a:50, misspelling. fosicithorax, Shannon, 1927a:6, misspelling. 
FUNEBRIS Hull, 1944b:11. Type-locality: Guyana, Essequibo River, Moraballi Creek. Distr. - same. Type o BM(NH).

HINEI Thompson (nom. nov. for transversa Hine). Distr. - Honduras. NOM. NOV.

transversa Hine, 1913:208 (Myiolepta; preocc. Walker, 1857). Typelocality: Honduras, Puerto Cortez. Type o OhSU.

LATITARSATA (Macquart), 1842:133 (73), pl. 14, fig. 1 (habitus), 1a (head) (Xylota). Type-locality: "?South America". Distr. — same. Type o MNHN. N. COMB.

Panamensis Curran, 1930f:10. Type-locality: Panama, Canal Zone, Barro Colorado Island. Distr. - same. Type o $\mathrm{AMNH}^{*}$.

Rudrs Hull, 1944j:43. Type-locality: Panama, Changuinola Dist. Distr. same. Type ơ CU.

SCUtellata Curran, 1934b:465. Type-locality: Guyana, Kartabo. Distr. same. Type $\sigma^{*}$ AMNH*.

SPINosA (Shannon), 1925a:109 (Zonemyia). Type-locality: Panama, Trinidad River. Distr. - same. Type o USNM.

TRANSVERSA (Walker), 1857:153 (Syritta). Type-locality: "Valley of Amazon". Distr. - same. Type ơ BM(NH).

\section{Genus Macrometopia Philippi}

Macrometoria Philippi, 1865:740. Type-species, atra Philippi (mon.).

ATrA Philippi, 1865:740. Type-locality: Chile, Corral. Distr. - Chile (Santiago - Bío Bío), Argentina (Neuquén). Type(s) ? MNHNS. Ref. - Hull, 1949d:365, fig. 22i (head).

\section{Genus Sterphus Philippi}

STERPHUS Philippi, 1865:737. Type-species, autumnalis Philippi (mon.) = coeruleus Rondani.

Stherphus, Fluke, 1957:106, misspelling.

\section{Subgenus Sterphus Philippi}

AURIFrons Shannon, 1926a:46. Type-locality: Chile, Juan Fernández Islands. Distr. - same. Type o BM(NH).

COeruleus (Rondani), 1863:8 (Xylota). Type-locality: "Chile". Distr. Chile, Argentina (Neuquén). Type $\sigma^{*}$ MIZUN. Ref. - Shannon \& Aubertin, 1933:155, fig. 33 (head).

autumnatis Philippi, 1865:782. Type-locality: Chile, Prov. Valdivia. Type(s) ? MNHNS.

antennalis Philippi, 1865:737, incorrect original spelling (1st revisor, Lynch Arribálzaga, 1892b:191).

aurifacies Schiner, 1868:360 (Xylota). Type-locality: "Chile". Types ? VMNH*. N. SYN.

\section{Subgenus CREPIDomyIa Shannon}

CREPIDOMYIA Shannon, 1926a:47 (as genus). Type-species, tricrepis Shannon (orig. des.). 
Tatuomyia Shannon, 1926a:48 (as genus). Type-species, batesi Shannon (orig. des.).

Senoceria Hull, 1930:144 (as genus). Type-species, spinifemorata Hull (orig. des.) = coarctatus. Wiedemann.

Mutillimyia Hull, .1943b:139 (as genus). Type-species, Ceriogaster auricaudata Williston (orig. des.).

Willistonimyia Hull, 1943b:140, incorrect original spelling of Mutillimyia Hull.

AURICAUDATUS (Williston), 1892:73, pl. 2:10 (habitus), 10a (head) (Ceriogaster). Type-locality: Mexico, Guerrero, Omiltemi, $8000 \mathrm{ft}$. Distr. - same. Types of o $\mathrm{BM}(\mathrm{NH}) *$. N. COMB.

BATESI (Shannon), 1926a:48 (Tatuomyia), Type-locality: Brazil, Amazonas, Tefé (as Ega.) Distr. - same. Type ơ BM(NH). N. COMB.

ChLoRopyga (Schiner), 1868:366 (Xylota). Type-locality: "Columbien". Distr. - same. Types of o VMNH*. N. COMB.

COARCTATUS (Wiedemann), 1830:100 (Xylota). Type-locality: "Brazil". Distr. - Panama, Brazil. Type o SMF*. N. COMB.

spinifemoratus Hull, 1930:146, figs. 2 (mesonotum), 5 (head), 6 (hind leg) (Senoceria). Type-locality: "Brazil". Type o" ANSP*.

CYBele (Hull), 1951c:184 (Crepidomyia). Type-locality: Peru, Chanchamayo. Distr. - Colombia, Peru. Tyype \& Hull*. N. COMB.

GENUINUS (Williston), 1888:284 (Xylota). Type-locality: Brazil, Rio de Janeiro. Distr. - Peru, Brazil, Argentina (Tucumán). Type o AMNH*. Ref. - Fluke, 1950b:449, fig. (male genitalia). N. COMB.

Plagiatus (Wiedemann), 1830:98 (Xylota). Type-locality: "Brazil". Distr. - Guyana, Peru, Brazil (Amapá, Amazonas). Type o VMNH. N. COMB.

TINCTUS (Fluke), 1950b:453, figs. 20 (head), 21 (wing) (Crepidomyia). Type-locality: Brazil, Santa Catarina, Nova Teutônia. Distr. same. Type o AMNH*. N. COMB.

TRICREPIS (Shannon), 1926a:47 (Crepidomyia). Type-locality: Peru, Río Charape. Distr. - Cuba, Peru, Bolivia. Type ơ USNM*. N. COMB.

darlingtoni Hull, 1944c:40 (Crepidomyia). Type-locality: Cuba, Eastern Oriente, Mounts north of Imias, 3-4000 ft. Type o MCZ*. N. SYN.

?dion Hull, 1951c:183 (Crepidomyia). Type-locality: Peru, Chanchamayo. Type $q$ Hull*. N. SYN.

\section{Genus Odyneromyia Shannon \& Aubertin}

ODYNEROMYia Shannon \& Aubertin, 1933:156. Type-species, Doros odyneroides Philippi (orig. des.).

ODYNEROIDES (Philippi), 1865:747 (Doros?). Type-locality: Chile, Corral. Distr. - Chile, Argentina (Neuquén). Type(s) ? MNHNS.

VALDIVIFORMIS Shannon \& Aubertin, 1933:157. Type-locality: Argentina, Peulla. Type or USNM.

Genus VALdiviomyia Vockeroth, nom. nov.

Valdivia Shannon, 1927a:31 (preocc. White, 1847; Ragonot, 1888). Typespecies, darwini Shannon (orig. des.).

Voldivia, Shannon, 1927a:32, incsrrect original spelling [in combination of Voldivia darwini]. 
VALDIVIOMYiA Vockeroth, nom. nov. for Valdivia Shannon. Type-species, Valdivia darwini Shannon (aut.).

Ref. - Sedman, 1965 (key).

CAMRASI (Sedman), 1965:199, figs. 5 (head), 6-8 (male genitalia) (Valdivia). Type-locality: Chile, Icalma. Distr. - same. Type ot USNM. N. COMB.

DARWINI (Shannon), 1927a:32 (Voldivia). Type-locality: Chile, Valdivia. Distr. - Chile (Bío Bío - Chiloé). Type o BM(NH). Ref. Sedman, 1965:197 (description of male), figs. 1-2 (head), 3-4 (male genitalia). N. COMB.

EDWARDSI (Shannon \& Aubertin), 1933:150 (Valdivia). Type-locality: Argentina, Puerto Blest. Distr. - same. Type o BM(NH). N. COMB.

Nigra (Shannon), 1927a:32 (Valdivia). Type-locality: Chile, Santiago. Distr. - same. Type ơ USNM. N. COMB.

RUficauda (Shannon), 1927a:32 (Valdivia). Type-locality: Chile, Santiago. Distr. - same. Type o USNM. N. COMB.

Valdiviana (Philippi), 1865:748 (Ocyptamus?). Type-locality: Chile, Valdivia. Distr. - Chile. Types o $\sigma^{7}$ MNHNS. N. COMB.

albimanus Bigot, 1883b:325 (Ocyptamus). Type-locality: "Chile". Type of $\mathrm{BM}(\mathrm{NH}) *$. N. SYN.

\section{Genus ANERIOpHora Stuardo \& Cortés}

Eriophora Philippi, 1865:735 (preocc. Simon, 1864). Type-species, aureorufa Philippi (mon.).

ANERIOPHORA Stuardo \& Cortés, 1952:311 (nom nov. for Eriophora Philippi). Type-species, Eriophora aureorufa (Philippi) (aut.).

AUREORUFA (Philippi), 1865:736, fig. 36 (habitus) (Eriophora). Type-locality: Chile, Valdivia Prov. Distr. - Chile (Malleco - Aysén). Types $\sigma^{7}$ o MNHNS. Ref. - Hull, 1949d:365, figs. 22a (habitus, hind leg), 22b (antenna), 22c (head).

\section{Genus Flukea Etcheverry}

FLUKEA Etcheverry, 1966:2. Type-species, vockerothi Etcheverry (orig. des.).

Fluckea Etcheverry, 1966:2, incorrect original spelling of Flukea.

vOCKEROTHI Etcheverry, 1966:3, figs. 1-35 (all aspects). Type-locality: Chile, Chillán. Distr. - Chile (Ñuble - Ausén). Type ơ UCSC.

\section{Genus Criorhina Meigen}

Penthesilea Meigen, 1800:35. Type-species, Musca ruficauda De Geer (sub. mon., Bezzi, in Hendel, 1908:67; misid.) = ranunculi Panzer. Suppressed by I.C.Z.N., 1963:339.

CRIORHINA Meigen, 1822:236. Type-species, Syrphus asilicus Fallén (Westwood, 1840:136).

Eurhinomallota Bigot, 1882a:1xvii. Type-species, metallica Bigot (mon.). Romaleosyrphus Bigot, 1882a:cxxix. Type-species, villosus Bigot (mon.). N. SYN.

ARCTOPHILOIDES (Giglio-Tos), 1892c:7 (Crioprora). Type-locality: Mexico, Angang[ueol]. Distr. - Mexico (Durango, Michoacán, Distrito Federal). Types o 0 UTOR*. 
tapeta Fluke, 1939:369, fig. 7 (head). Type-locality: Mexico, Mexico City, 10,000 ft. Type of AMNH*. N. SYN.

Metallica (Bigot), 1882a:lxvii (Eurhinomallota). Type-locality: "Mexico". Distr. - same. Type o OXF*.

villosa (Bigot), 1882a:cxxix (Romaleosyrphus). Type-locality: Mexico. Distr. - same. Type of BM(NH)*. N. COMB.

\section{Genus Philippimyia Shannon}

PHILIPPIMYIA Shannon, 1926a:47. Type-species, ?Sterphus cyanocephalus Philippi (orig. des.).

CYanocephala (Philippi), 1865:738 (Sterphus?). Type-locality: Chile, Santiago. Distr. - Chile (Valparaíso - Malleco). Type ? MNHNS.

\section{Genus Blera Billberg}

BLERA Billberg, 1820:118. Type-species, Musca fallax Linnaeus (Johnson, 1911:73). Ref. - Curran, 1953:12 (key).

Pallipes (Bigot), 1883b:352 (Spilomyia). Type-locality: "Mexico". Distr. same. Type \& $\mathrm{BM}(\mathrm{NH}) *$. N. COMB.

\section{Genus SyritTa Lepeletier \& Serville}

SYRITTA ‘.epeletier \& Serville, in Latreille, et al., 1828:808 (as a subgenus). Type-species, Musca pipiens Linnaeus (mon.).

A ustrosyritta Marnef, 1967:268. Type-species, cortesi Marnef (orig. des.) = flaviventris Macquart.

FLAVIVEnTRIS Macquart, 1842:135 (75). Type-locality: "Senegal". Distr. Africa, southern Europe; Chile, Argentina, Brazil. Type o MNHN*. cortesi Marnef, 1967:269, fig. b (4th sternum of male) (Austrosyritta). Type-locality: Chile, Valparaíso, Con-Con. Type or UCVC.

PIPIENS (Linnaeus), 1758:594 (Musca). Type-locality: "Europe". Distr. Holarctic, Asia Minor, North Africa; Mexico (Durango, Chihuahua). Type ? ? Ref. - Williston, 1887b:240 (description); pl. $12: 1$ (wing), 1a (hind leg), $1 b+c$ (head).

\section{Genus Senogaster Macquart}

SENOGASTER Macquar仓, 1834:519. Type-species, coerulescens Macquart (mon.) $=$ dentipes Fabricius.

Acrochordonodes Bigot, 1878:xcii. Type-species, vittatus Bigot (mon.) = dentipes Fabricius.

Stenogaster, Agassiz, 1846:338, 351, emendation.

DENTIPES (Fabricius), 1787:338 (Syrphus). Type-locality: "Cajennae". Distr. - Surinam, Brazli. Type ? Kiel [destroyed, Zimsen, 1964:473]. Ref. - Williston, 1887b:239 (description), pl. 11:1 (habitus), 1a (wing), 1b (head); Hull, 1949d:366, figs. 23j (habitus), 23k (hind leg), 231 (abdomen), 23m (head).

lineata Fabricius, 1805:194 (Milesia). Type-locality: "America meridionali". Type $+\mathrm{MC}^{*}$.

coerulescens Macquart, 1834:519. Type-locality: "Exotique". Type o OXF*. 
vittata Bigot, 1878:xcvii (Acrochordonodes). Type-locality: "Cayenne". Type $\$$ OXF*.

comstocki Williston, 1882b:326. Type-locality: U.S.A., New York [error]. Type or USNM.

var. RUfofemorata Doesburg, 1966:97. Type-locality: Surinam, Paramaribo \& Houttuin. Distr, - same. Types of $q$ Doesburg.

\section{Genus Tropidia Meigen}

TROPIDIA Meigen, 1822:346. Type-species, Eristalis milesiformis Fallén (Curtis, 1832:pl. 401) = scita Harris.

Ortholophus Bigot, 1882a:cxxix. Type-species, notatus Bigot (mon.). Ref. - Shannon \& Aubertin, 1933:152 (key).

Flavimana Philippi, 1865:745. Type-locality: Chile, Santiago. Distr. Chile, Argentina (Mendoza). Type $\sigma^{*}$ MNHNS.

INSULARIS Lynch Arribálzaga, 1892a:238 (1892c:94). Type-locality: Argentina, "Insula Antequera". Distr. - same. Types ot 아 MACN.

NIGRICORNIS Philippi, 1865:745. Type-locality: Chile, Santiago. Distr. Chile. Type o MNHNS.

bivittata Bigot, 1884a:547 (Xylota). Type-locality: "Chile". Type " $\mathrm{BM}(\mathrm{NH})$.

Notata (Bigot), 1882a:cxxix (Ortholophus). Type-locality: "Chile". Distr. - Chile, Argentina. Type $\sigma^{*} \mathrm{BM}(\mathrm{NH}) *$

PUlchra Hull, 1944j:52. Type-locality: Argentina, Mendoza, Potrerillos. Distr. - same. Type on CU.

RUBRICORNIS Philippi, 1865:744. Type-locality: Chile, Colchagua Prov. Distr. - same. Type o MNHNS.

\section{Genus Milesia Latreille}

MILESIA Latreille, 1804:194. Type-species, Syrphus crabroniformis Fabricius (Williston, 1887b:254) to preserve established usage. [see Thompson, 1972:176]. Ref. - Hull, 1924 (key).

BeLla Townsend, 1897e:142. Type-locality: U.S.A., New Mexico, Rio Ruidoso, 4 miles west of Dowling's Mill, $6600 \mathrm{ft}$. Distr. - U.S.A. (Arizona, New Mexico); Mexico (Durango). Types o f $\mathrm{BM}(\mathrm{NH})$.

NIGRA Fluke, 1939:371, fig. 11 (abdomen). Type-locality: Panama, Chiriquí, El Volcán. Distr. - same. Type ơ AMNH*.

PULchra Williston, 1892:74, pl. 2:11 (habitus), 11a (head). Type-locality: Guatemala, Purula; Vera Paz, Senahú. Distr. - same. Types ${ }^{*}$ $\mathrm{BM}(\mathrm{NH})$.

SCUtellata Hull, 1924:280. Type-locality: U.S.A., Mississippi, Mississippi Agricultural \& Mechanical College. Distr. - U.S.A. (Louisana to North Carolina, south to Florida); Panama (Canal Zone). Type o Hull.

virginIENSIS (Drury), 1773:71, pl. 37, fig. 6 \& index, p. 2 (Musca). Typelocality: U.S.A., Virginia. Distr. - U.S.A. (Minnesota) to Canada (Ontario), south to U.S.A. (Texas \& Florida); Mexico (Nuevo León), ?Guadeloupe [Miacquart, 1842:141 (81)]. Type ? ? 


\section{Genus Spilomyia Meigen}

SPILOMyia Meigen, 1803:273. Type-species, Musca diophthalma Linnaeus (Coquillett, 1910b:617). Ref. - Curran, 1951 (key).

EPHYPpium (Osten-Sacken), 1875:70 (Mixtemyia). Type-locality: "Mexico". Distr. - same. Type o MCZ*. Ref. - Williston, 1887b:249 (redescription).

Gratiosa Wulp, 1888:372. Type-locality: Argentina, Tucumán Prov. Distr. - Brazil (São Paulo), Argentina (Tucumán). Type $q$ lost?

KAHLI Snow, 1895b:245. Type-locality: U.S.A., New Mexico, Magdalena Range, "near summit of 'Little Baldy" ". Distr. - U.S.A. (Arizona, Texas, New Mexico); Mexico (Chihuahua). Type o UK [Byers, et al., 1962:168].

LONGICORNIS Loew, 1872a:82 (1872b:258). Type-locality: U.S.A., Massachusetts, Pennsylvania, Texas. Distr. - Canada (Quebec) west to U.S.A. (Minnesota, south to Texas \& Florida); Mexico [Wirth, et al., 1965:613]. Types o o MCZ*.

OBSCURA Coquillett, 1902:195. Type-locality: Mexico, Chihuahua, Sierra Madre, Head of Rio Piedras Verdes, $7300 \mathrm{ft}$. Distr. - same. Types ot of USNM.

PleUralis Williston, 1887b:247. Type-locality: "Mexico". Distr. - same. Type ơ MCZ*.

\section{Genus Strlbosoma Philippi}

Stilbosoma Philippi, 1865:736. Type-species, rubiceps Philippi (Shannon, 1926a:45).

CYANEUM Philippi, 1865:736. Type-locality: Chile, Santiago \& Valdivia Provs. Distr. - Chile (Santiago - Aysén), Argentina (Neuquén). Types ? MNHNS. Ref. - Shannon \& Aubertin, 1933:154, figs. 31 (head), 32 (wing).

nigrinervis Philippi, 1865:737. Type-locality: Chile, Valdivia Prov. Type ? MNHNS.

nigricorne, Williston, 1886:322, misspelling.

RUBICEPS Philippi, 1865:738. Type-locality: Chile, Santiago. Distr. - Chile (Santiago - Cautin). Type ? MNHNS.

ruficeps, Williston, 1886:322, misspelling.

\section{Genus Hemixylota Shannon \& Aubertin}

HEMIXYLOTA Shannon \& Aubertin, 1933:146. Type-species, varipes Shannon \& Aubertin (orig. des.). Ref. - Shannon \& Aubertin, 1933: 146 (key).

INCERTA Shannon \& Aubertin, 1933:148. Type-locality: Argentina, Lake Nahuel Huapí, Puerto Blest. Distr. - same. Type o BM(NH).

UNICOLOR Shannon \& Aubertin, 1933:148. Type-locality: Argentina, Ensenada. Distr. - Chile (Malleco), Argentina (Neuquén). Type f $\mathrm{BM}(\mathrm{NH})$.

VARIPES Shannon \& Aubertin, 1933:146, fig. 30 (head). Type-locality: Argentina, Puerto Blest. Distr. - Argentina (Neuquén). Type ó $\mathrm{BM}(\mathrm{NH})$ 


\section{Unplaced species of XYLOTINI}

MONTENSIS Hull, 1938:122, figs. 1 (head), 12 (antenna) (Nosodepus). Type-locality: Venezuela, Aragua, Colonia Tovar, 6000-7000 ft. Distr. - same. Type o CM.

\section{UnReCognized Neotropical Syrpidae}

\section{Genus PIA Philippi}

PIA Philippi, 1865:742. Type-species, cyanea Philippi (mon.).

CYANEA Philippi, 1865:742, fig. 40 (habitus), 40a (head). Type-locality: Chile, Illapel. Type or MNHNS (lost?-FCT).

\section{UNRECOGNIZED SPECIES}

CHIRAGRA Fabricius, 1805:198 (Merodon). Type-locality: "America meridionali". Type ? lost (Zimsen, 1964:474).

CYANESCENS Macquart, 1834:511 (Helophilus). Type-locality: "Brazil". Type ? MNHN (not found - JRV).

FLAVA Sack, 1941:117 (Ceratophya). Type-locality: Peru, Rosalina on Rio Urubamba. Type o $\mathrm{MD}$.

Gagathina Bigot, 1887: lxxix (Priomerus). Type-locality: Ecuador, Loja. Type o ? (not found at either OXF or BM(NH) - JRV \& FCT).

Nubeculosus Wulp, 1888:372 (Pterallastes). Type-locality: Argentina, Prov. Tucumán. Type $\sigma^{*}$ lost?

\section{LITERATURE CITED}

AgASSIZ, L.

1846. Nomenclatoris zoologici Index Universalis, continens nomina systematica clessium, ordinum, familiarum et generum animalium omnium, tam viventium quam fossilium, secundum ordinem alphabeticum unicum disposita, adjectis homonymis plantarum, nec non variis adnotationibus et emendationibus, viii +393 pp. Soloduri $[=$ Solothurn, Switzerland $]$

ALDRICH, J. M.

1905. A catalogue of North American Diptera. Smithsn. Misc. Collect. 46(2): 1-680.

1933. Notes on Diptera, No. 6. Proc. ent. Soc. Wash. 35: 165-170.

ANDERSSON, H.

1970. Taxonomic Notes on the Genera Platycheirus and Melanostoma (Dipt., Syrphidae) with lectotype designations. Ent. scand. 1(3): 236-240.

D'Andretta, Maria A. V. \& Carrera, M.

1952. Resultados de uma expedição científica ao território do Acre. - Diptera. Papéis Avulsos Zool. 10(17): 293-306. 
ARNAUD, P. H., Jr.

1963. Records of Diptera from Guadalupe Island, Mexico. Second Paper. Ent. News 74: 117-129 (syrphids, p. 120).

AUsten, E. E.

1893. Description of New Species of Dipterous Insects of the Family Syrphidae in the British Museum with notes on species described by the late Francis Walker. Proc, zool. Soc. London 61: $131-164$

1913. Diptera from the Falkland Islands, with Descriptions of a new Genus and two new species. Ann. Mag. nat. Hist. (8)11: 498-504

BARRETTO, M. P. \& LANE, J.

1947. Novos Microdontinae brasileiros (Dipiera, Syrphidae). Rev. Ent., Rio de Janeiro, 18: 139-148.

BEAN, J. L.

1949. A study of male hypopygia of the species of Tubifera (Syrphidae, Diptera) that occur north of Mexico. Canad. Ent. 81: $140-152$

BECKER, T.

1903. Ägyptische Dipteren. Mitt zool. Mus. Berlin 2: 67-195, 4 taf.

BEZZI, M.

1908. Nomenklatorisches über Dipteren. Wien. Ent. Ztg. 27: 291-295.

1910. Zwei neue südamerikanische Microdon-Arten. Wien. Ent. Ztg. 29: 319-320.

Bigot, J. M. F.

1857a. Diptères nouveaux provenant du Chili. Ann. Soc. ent. France (3) $5: 277-308$

1857b. Dipteros. Pp. 328-349. In Sagra, R. de la, Historia fisica, politica y natural de la Isla de Cuba. Vol. 7, 371 pp. Paris, (see Stone, et al., 1965:1405).

1859. Dipterorum aliquot nova genera. Rev. Mag. Zool. (2)11: 305-315

1875. Diptères nouveaux ou peu connus. $5^{\mathrm{e}}$ partie, VI: Espèces exotiques nouvelles des genres Sphixea (Rondani) et Volucella auctorum. Ann. Soc. ent. France (5) 5: 469-482.

1878. Diagnose d'un nouveau genre de Diptères de la tribu des Syrphides. Ann Soc. ent. France, (5) 8(Bull.): xcvii.

1880a. Genres Plagiocera (Macq.), Formosia (Guérin) et Rutilia (Rob.-Desv.). Ann. Soc. ent. France, (5) 10: 85-89. 
1880b. Diptères nouveaux ou peu connus. 14e partie, XXI: Syrphldi (mihi). Genre Eristalis (Fabr.). Ann. Soc. ent. France, (5) 10: $213-230$.

1882a. Descriptions de quatre genres nouveaux de la tribu des Syrphides auctorum, ainsi que celles de deux nouvelles espèces. Ann. Soc. ent. France, (6) 2(Bull.): 1xvii-1xviii.

1882b. Diagnoses de Genres et espèces inédits de Syrphides. $1^{\text {re }}$ partie; $2^{\mathrm{e}}$ partie; $3^{\mathrm{e}}$ partie. Ann. Soc. ent. France, (6) 2 (Bull.): exiv-cxv; exx-cxxi; exxviii-cxxix.

1883a. Description d'un nouveau genre de Diptères de la tribu de Syrphides. Ann Soc. ent. France, (6) 3(Bull.): xx-xxi.

1883b. Diptères nouveaux ou peu connus. 20e partie, XXXI: Genres Volucella (Géoffr., Hist. des Insectes, 1764) et Phalachromyia (Rondani, Esame d. var. spec, d'Insetti Ditteri Brasiliani, Torino, 1848); 21e partie, XXXII: Syrphidi (1re partie); $22^{\mathrm{e}}$ partie, XXXII: Syrphidi (2e partie). Espèces nouvelles, no. I; Ann. Soc. ent. France, (6) 3: 61-88; 221-258; 315-356.

1884a. Diptères nouveaux ou peu connus. 23e partie, XXXII: Syrphidi (2e partie). Espèces nouvelles, no. II. Ann. Soc, ent. France, (6) $3: 535-560$.

1884b. Diptères nouveaux ou peu connus. 24e partie, XXXII: Syrphidi (2e partie). Espèces nouvelles, no. III. Ann. Soc. ent. France, (6) $4: 73-116$

1885a. Diptères nouveaux ou peu connus. 28 partie, XXXVI: Syrphidi. Addenda au mémoire publié dans les Annales de la Société entomologique de France (Anées 1883-1884). Ann. Soc. ent. France, (6) 4: 241-252.

1885b. [La description d'un nouveau genre de Diptères]. Ann. Soc. ent. France (6) 5: (Bull.) :clxxiii-clxxiv.

1887. Note sur divers Diptères de Yeso (Japan) et de Loja (Équateur): note suivie de la description de nouvelles espèces. Ann. Soc. ent. France, (6) 7 (Bull.): Ixxvi-1xxx.

1888a. Diptères nouveaux ou peu connus. $34^{\mathrm{e}}$ partie, XLII: Diagnoses de nouvelles espèces. Ann. Soc. ent. France, (6) 8: 253-270.

1888b. Mission scientifique du Cap Horn, 6, Zool. Dipt.: 1-45.

BILLBERG, G. J.

1820. Enumeratio insectorum in Museo Gust. Joh. Billberg. 138 pp. [Stockholm].

BLANCHARD, E.

1852. Orden IX. Dipteros. VI Sirfianos, pp. 403-413.1 In Gay, C., ed., Historia fisica y politica de Chile. Zoologia, Vol. 7, 471 pp. (see Stone, et al., 1965:1241). 
Blanchard, E. E.

1938. Descripciones y anotaciones de Dipteros argentinos. An. Soc. cient. Argentina 126: 345-386.

BRÈTHES, J.

1905 Insectos de Tucumán. An. Mus. Nac. Buenos Aires (3) 4: $32 \mathrm{~S}-347$.

1907. Catalogo de los Dipteros de las Republicas del Plata. $A n$. Mus. Nac. Buenos Aires (3) 9: 277-305. (syrphids, p. 291-294).

1909. Masarygidae, una nueva familia de dipteros. An. Mus. Nac. Buenos Aires (3) 10: 439-443.

1914. Description d'un nouveau Syrphide de la Republique Argentine. An. Mus. Nac. Buenos Aires (3) 26: 97-98. (12 May 1914).

1918. Quelques Diptères du Chili. Rev. Chil. Hist. Nat. 22: 50-51.

1919. Cueillette d'insectes au Rio Blanco. Rev. Chil. Hist. Nat. 22: 40-44. (syrphids, p. 42).

1920. Insectes du Perou. An. Soc. cient. Argentina 89: 27-54.

1922. Himenópteros y Dípteros de varias procedencias. An. Soc. cient. Argentina 93: 119-146.

1937. Nuevas especies peruanas de fauna y flora. Bol. Mus. Hist. Nat. "Javier Prado" 1: I-XVI.

\section{BrucN, CarLos}

1919. Observaciones biológicas sobre Temnocera spinigera Wied. (Diptera-Syrphidae). Rev. Mus, La Plata 24: 176-181.

1923. Observaciones biológicas acerca de "Salpingogaster nigriventris" Bigot (Diptero-Sírfido), Rev. Soc. Argent, cienc, nat. $i$ : 1-6.

Byers, G. W.; F. Blank; W. J. Hanson; D. F. Benway \& R. W. FreDRICHSON

1962. Catalogue of the Types in the Snow Entomological Museum. Part III. Univ. Kansas Sci. Bull 43 (5): 131-181 (167-169).

CAMPOS, F.

1960. Las moscas (Brachycera) del Ecuador. Rev. Ecuatoriana Hig. Med. Trop. 17(1): 1-99.

CAPElle, K. J.

1956. The genus Rhopalosyrphus, with a description of a new species from Arizona. J. Kans. ent. Soc. 29: 170-175.

CARRERA, M.

1940. Descrição do alótipo de Baccha lanei Curran, 1936. (Syrphidae, Diptera). Papéis Avulsos Zool. 1: 65-58. 
CarRera, M., \& Lenko, K.

1958. Descrição de duas espécies novas de Mixogaster (Diptera, Syrphidae) e observações sobre o inquilinismo de uma delas em ninhos de Iridomyrmex humilis, a "formiga argentina"'. Studia Ent. 1: 465-486.

Carrera, M., Lopes, H. de S., \& Lane, $J$.

1947a. Um novo gênero e duas espécies de Microdontinae (DipteraSyrphidae). Brasil-Médico 61: 3-5.

1947b. Contribuição ao conhecimento dos Microdontinae neotrópicos e descrição de duas novas espécies de Nausigaster Williston (Diptera, Syrphidae). Rev. Brasil. Biol. 7: 471-486.

Cendrero, Luis

1971. Zoologia Hispanoamericana. Invertebrados, i-xxi + 1151 pp. Mexico City.

Ceresa, L.

1934. Tre nuove specie de Quichuana Knab (Diptera Syrphidae). Atti Soc. Ital. Sci. Nat. 73: 383-392.

Cockerell, T. D. A.

1894. A Supplementary Note to Mr. Johnson's List of Jamaican Diptera. Proc. Acad. nat. Sci., Philadelphia 46: 419-420. (419).

COE, R. L.

1953. Diptera. Family Syrphidae. Handb. Ident. Brit. Ins. 10(1), $98 \mathrm{pp}$.

Colin, J. E.

1920. Eumerus strigatus Fallén and tuberculatus Rondani (Diptera, Syrphidae). Ent. mon. Mag. (3) 6: 102-106, pl. III.

1952. On the subdivision of the genus Pipizella Rnd., and an additional British species (Diptera, Syrphidae). J. Soc. Brit. Ent. 4: $85-88$.

Coquillett, D. W.

1900. Report on a collection of Dipterous insects from Puerto Rico. Proc. U. S. natn. Mus. 22: 249-270. (252-253).

1901. Papers from the Hopkins Galapagos Expedition, 1898-1899. II. Entomological Results (2): Dipteria (sic). Proc. Wash. Acad. Sci. 3: 371-379.

1902. New Cyclorhaphous Diptera from Mexico and New Mexico. Canad. Ent. 34: 195-202.

1910a. The type-species of the North American genera of Diptera. Proc. U. S. nat. Mus, 37: 499-647.

1910b. Corrections to my paper on the type-species of the North American genera of Diptera. Canad. Ent. 42: 375-379. 
CURRan, C. H.

1925a. Contribution to a monograph of the American Syrphidae from North of Mexico. Kansas U. Sci. Bull. (1924) 15: 7-216.

1925b. New American Diptera. I. Ann. Mag. nat. Hist. (8)16: 243-253.

1925c. New American Diptera. II. Ann. Mag. nat. Hist. (9) 16: 338-354

1925d. New Diptera in the American Museum of Natural History. Amer. Mus. Novit. 176: 1-10.

1926a. Partial synopsis of American species of Volucella with notes on Wiedemann's types. Ann. Ent. Soc. Amer. 19: 50-66.

1926b. Notes on Wiedemann's types of Syrphidae. Canad. Ent. 58: 111-115.

1926c. Appendix. New Diptera from Jamaica. pp. 102-114. In Gowdey, C. C. Catalogus insectorum jamaicensis. Ent. Bull., Jamaica Dept. Agr. 4 (1/2): 1-114.

1927a. Synopsis of the syrphid genus Copestylum Macq. (Diptera). Ent. News 38: 43-46.

1927b. New Neotropical and Oriental Diptera in the American Museum of Natural History. Amer. Mus. Novit. 245: 1-9.

1927c. Four new species of Volucella (Syrphidae, Dipt.), Bull. Brooklyn Ent. Soc. 22(2): 84-88.

1928a. Insects of Porto Rico and the Virgin Islands. Diptera or two winged flies. Pt. 1, pp. 1-118. In New York Acad. of Sci., Scientific Survey of Puerto Rico and the Virgin Islands. Vol. 11.

1928b. Records and descriptions of Diptera mostly from Jamaica. pp. 29-45. In Appendix of Gowdey, C. C., 1928, Catalogus Insectorum jamaicensis. Ent. Bull., Jamaica Dept. Agric. 4(3): $1-45$ (q.v.).

1929. New Syrphidae and Tachinidae. Ann. Ent. Soc. Amer. 22: 489-510.

1930a. New Diptera belonging to the Genus Baccha Fabricius (Syrphidae). Amer. Mus. Novit. 403: 1-16.

1930b. New Diptera belonging to the Genus Mesogramma Loew (Syrphidae). Amer. Mus. Novit. 405: 1-14.

1930c. New species of Eristalinae with notes (Syrphidae, Diptera). Amer. Mus. Novit. 411: 1-27. 
1930d. New species of Volucellinae from America (Syrphidae, Diptera). Amer. Mus. Novit. 413: 1-23.

1930e. New Diptera from North and Central America. Amer. Mus. Novit. 415: 1-16.

1930f. New Syrphidae from Central America and the West Indies. Amer. Mus. Novit. 416: 1-11.

1932a. New American Syrphidae (Diptera), with notes. Amer. Mus. Novit. 519: 1-9.

1932b. The Norwegian Zoological Expedition to the Galapagos Islands, 1925, conducted by Alf Wollebaek. IV. Diptera. Meddel. Zool. Mus., Oslo 30: 347-366.

1933. Two new Diptera from Guatemala. Amer. Mus. Novit. 643: 1-2.

1934a. The families and genera of North American Diptera, 512 pp. N.Y.

1934b. Diptera of Kartabo, Bartica District, British Guiana. Bull. Amer. Mus. Nat. Hist "66: 287-532.

1934c. The Templeton Crocker Expedition of the California Academy of Sciences, 1932. No. 13. Diptera. Proc. Cal. Acad. Sci. 21: 147-172

1935. New American Diptera. Amer. Mus. Novit. 812: 1-24.

1936a. Three new species of Meromacrus (Diptera, Syrphidae). Amer. Mus. Novit. 834: 1-5.

1936b. New Neotropical Syrphidae (Diptera). Amer. Mus. Novit. 882: 1-17.

1937. The Neotropical species of Melanostoma and allies (Syrphidae: Diptera). Amer. Mus. Novit. 926: 1-4.

1938. New American Diptera, Amer. Mus. Novit. 975: 1-7.

1939a. Synopsis of the American species of Volucella (Syrphidae; Diptera). Part I. Table of Species. Amer. Mus. Novit. 1027: 1-7.

1939b. Synopsis of the American species of Volucella (Syrphidae; Diptera). Part II. Descriptions of New Species. Amer. Mus. Novit. 1028: 1-17.

1939c. Two new American Diptera with notes on Asemosyrphus Bigot. Amer. Mus. Novit. 1031: 1-3.

1939d. New Neotropical Baccha Fabricius (Syrphidae: Diptera). Amer. Mus. Novit. 1041: 1-12.

1939e. Syrphidae (Diptera). In Résultats scientifiques des croisières du Navire-Ecole Belge "Mercator", Vol. 2:173-175. Mem. Mus. R. Hist. nat. Belg. (2) 15. 
1940. Some new Neotropical Syrphidae (Diptera). Amer. Mus. Novit. 1086: 1-14.

1941. New American Syrphidae. Bull. Amer. Mus. Nat. Hist. 78: 243-304.

1947a. A fissicorn syrphid fly from Brazil (Diptera). Amer. Mus. Novit. 1347: 1-2.

1947. New species of Volucella from Hawaii and the United States (Syrphidae, Diptera). Amer. Mus. Novit. 1361: 1-6.

1951. Synopsis of the North American species of Spilomyia. (Syrphidae, Diptera). Amer. Mus. Novit. 1492: 1-11.

1953. Notes and description of some Mydaidae and Syrphidae. Amer. Mus. Novit. 1645: 1-15.

Curtis, J.

1832-1839. British entomology: Being illustrations and descriptions of the genera of insects found in Great Britain and Ireland. Vol. 9, pls. 384-433, 1832; Vol. 13, pls. 626-673, 1837; Vol. 16, pls. 722-769, 1839. London.

DAVIDSON, W. M.

1922. Notes on certain species of Melanostoma (Diptera: Syrphidae). Trans. Amer. ent. Soc. 48: 35-47.

DeGeer, C.

1776. Mémoires pour servir à l'histoire des Insectes. Vol. 6, 523 pp. Stockholm.

DOESBURG, P. H. VAN

1958. Syrphiden-allerlei. Ent. Ber., Amsterdan 18: 41-46.

1962. Preliminary list of Syrphidae known from Suriname and British and French Guiana, Stud. Fauna Suriname (Natuurwet. Stud. Suriname) The Hague 5 (28): 1-33.

1966. Syrphidae from Suriname. Additional records and descriptions. Stud. Fauna Suriname (Natuurwet. Stud. Suriname), 9 (35): 61-107.

1970. Records of Syrphidae from the Lesser Antilles. Studies on the Fauna of Curaçao and other Caribbean Islands 34 (126): 90-101.

DRURY, D.

1770.* Illustrations of natural history. Wherein are exhibited upwards of 240 figures of exotic insects, according to their. different genera. Vol. 1, 130 pp., 4 fig., 60 pls. London.

1773.* Illustrations of natural history. Wherein are exhibited upwards of 240 figures of exotic insects, according to their different genera. Vol. 2, $90 \mathrm{pp}$., $50 \mathrm{pls}$., and index to Vols 1-2. (4 unnumbered pages), London. 
* the name of the insects figured are found only in the index; thus the names must date from 1773. See Stone et al., $1965: 1207$.

\section{DUSEK, J. \& P. LÄSKA}

1967. Bersuch zum Aufbau eines natürlichen Systems mitteleuropäischer Arten der Unterfamilie Syrphinae (Diptera). Acta Sci. nat., Brno 1: 349-390.

ENDERLEIN, G.

1912. Die Insekten des Antarkto-Archiplata-Gebietes (Feuerland, Falklands-Inseln, Süd-Georgien). 20. Beitrag zur Kenntnis der antarktischen Faune. Kungl. Svenka Vet.-Akad. Handl. 48(3), 170 pp., 4 tafeln.

1938. Beiträge zur Kenntnis der Syrphiden. Sitzber, Gesell. Naturf. Freunde, Berlin, 1937: 192-237.

1940. Die Dipterenfauna der Juan-Fernandez-Inseln und der OsterInsel. In. Skottsberg, Natural History of Juan Fernandez and Easter Isiand. Zoology 3(5): 643-680.

ETCHEVERRY, M.

1963. Descripciones originales, sinonimia y distribución geográfica de las especies de la familia Syrphidae (Diptera) en Chile. Publ. Centro Estudios Ent. 5: 1-141.

1966. Flukea vockerothi nuevo genero y nueva especie de Syrphidae Chileno (Diptera). Publ. Centro Estudios Ent. 8: 1-22.

Etcheverry, M. \& R. D. Shenefelt

1962. A preliminary study of the genitalia of Chilean Syphidae. Verhandl. XI. Internat. Kongr. Ent., 1960, 1:207-214, figs.

FABRICIUS, J. C.

1775. Systema entomologiae, sistens insectorum classes, ordines, genera, species adiectis synonymis, locis, descriptionibus, observationibus. 832 pp. Flensburgi et Lipsiae $[=$ Flensburg and Leipzig].

1781. Species insectorum exhibentes eorum differentias specificas, synonyms, auctorum, loca natalia, metamorphosin. Vol. 2. 517 pp. Hamburgi et Kilonii [=Hamburg and Kiel].

1787. Mantissa insectorum sistens species nuper detectas. Vol. 2. 382 pp. Hafniae [=Copenhagen].

1794. Entomologia systematica emendata et aucta. Vol. 4. 472 pp. Hafniae [ = Copenhagen].

1798. Supplementum entomologiae systematicae. 572 pp. Hafniae [ = Copenhagen $]$.

1799. Index alphabeticus in J. C. Fabricii Supplementum Entomologiae Systematicae. 53 pp. Hafniae [=Copenhagen]. 
1805. Systema antliatorum secundum ordines, genera, species. 373 p., +30 pp. Brunsvigae $[=$ Brunswick $]$.

FALLÉN, C. F.

1810. Specim. entomolog. novam Diptera disponendi methodum exhibens. $26 \mathrm{pp} ., 1 \mathrm{pl}$. Lund.

1816. Syrphici Sveciae. Pp. 1-22 (cont.). Lundae (=Lund).

1817. Syrphici Sveciae. (Concl.). Pp. 23-62. Lundae (=Lund).

FLUKe, C. L.

1936. New Syrphidae (Diptera) from Brazil and Cuba. J. Kans. Ent. Soc. 9: 59-65.

1937. New South American Syrphidae (Diptera), Amer. Mus, Novit. 941: 1-14.

1939. New Syrphidae (Diptera) from Central and North America. Ann. Ent. Soc. Amer. 32: 365-375.

1942. Revision of the Neotropical Syrphini related to Syrphus. Amer. Mus. Novit. 1201: 1-24:

1943. A new genus and new species of Syrphidae (Diptera) from Ecuador. Ann. Ent. Soc. Amer. 36: 425-431.

1945. The Melanostomini of the Neotropical Region (Diptera, Syrphidae). Amer. Mus. Novit. 1272: 1-29.

1950a. The male genitalia of Syrphus, Epistrophe and related genera (Diptera, Syrphidae). Trans. Wis. Acad. Sci. Arts Letters 40: 115-148.

1950b. Some new tropical syrphid flies with notes on others. Acta Zool。Lilloana 9: 439-454.

1951a. The genus "Dolichogyna" (Diptera, Syrphidae). Acta Zool. Lilloana 12: 465-478.

1951b. Syrphid flies related to Volucella scutellata Macquart. Amer. Mus. Novit. 1503: 1-33.

1951c. Corrections to the paper on "The male genitalia of Syrphus, Epistrophe and related genera" (Diptera: Syrphidae). Ent. News 62: 217-218.

1953a. New Syrphidae from North America. J. Kans. Ent. Soc. 26: 125-129.

1953b. Some Syrphid Fly Synonomy (sic). Ent. News 64: 208-209.

1955. Los Insectos de las Islas Juan Fernandez. 18. Syrphidae (Diptera). Rev. Chilena Ent. 4: 39-43. 
1956. Catalogue of the family Syrphidae in the Neotropical Region. Rev. Brasil Ent. 6: 193-268.

1957. Catalogue of the family Syrphidae in the Neotropical Region. Rev. Brasil Ent. 7: 1-181.

1958. A study of the male genitalia of the Melanostomini (DipteraSyrphidae). Trans. Wis. Acad. Sci. Arts Letters (1957) 46: 261-279.

1960. Concerning the Catalogue of Neotropical Syrphidae. Rev. Bras. Ent. 9: 169.

FLUKE, C. L., \& Hull, F. M.

1947. The Cartosyrphus flies of North America (Syrphidae). Trans. Wis. Acad. Sci. Arts, Letters, (1945) 37: 221-263.

FluKe, C. L. \& WEEMS, H. V., JR.

1956. The Myoleptini of the Americas (Diptera, Syrphidae). Amer. Mus. Novit. 1758: 1-23.

F'REY, R.

1946. Úber der Gattungen der Syrphiden-Unterfamilie Syrphinae (Syrphinae+Bacchinae). Notulae Ent. (1945) 25: 152-172.

Giglio-Tos, E.

1891. Diagnosi de quattro nuovi generi di Ditteri. Bol. Mus. Zool. Anat. Comp 6(108): 1-6.

1892a. Un nuovo genere di Sirfidi. Bol. Mus. Zool. Anat. Comp. 7 (117): 1-3.

1892b. Sui due generi di Sirfidi Rhopalosyrphus ed Omegasyrphus. Bol. Mus. Zool. Anat. Comp. 7(118): 1-3.

1892c. Diagnosi di nuove specie di Ditteri. VI. Sirfidi del Messico. Bol. Mus. Zool. Anat. Comp. Y(123): 1-7.

1892d. Diagnosi di nuove specie di Ditteri. VII. Sirfidi del Messico. Bol. Mus. Zool. Anat. Comp. 7(132): 1-5.

1892e. Ditteri del Messico. Pt. 1, 72 pp. Torino.

1893. Ditteri del Messico. Pt. 2, 80 pp. Torino.

GMELIN, J. F.

1790. Caroli à Linné, Systema naturae per regna tria naturae,... Ed. 13. Vol. 1: Regnum Animale, Pt. 5, pp. 2225-3020. Lipsiae (=Leipzig). (see Stone et al., 1965:1245).

GOFFE, E. R.

1946. The genera in Syrphidae of Lioy, 1864. J. Brit. Ent. 3(1): $27-30$. 
GOOT, V. S. VAN DER

1964. Fluke's catalogue of neotropical Syrphidae (Insects, Diptera) a critical study with an appendix on new names in Syrphidae. Beaufortia 10(127): 212-221.

GOWDEY, C. C.

1926. Catalogus insectorum jamaicensis. Ent. Bull., Jamaica Dept. Agric. 4(1): xiv +114 pp.; 4(2): $\mathrm{ii}+10$ pp. $(79-80)$.

1928. Catalogus insectorum jamaicensis. Ent. Bull., Jamaica Dept. Agric. 4(3): $\mathrm{iii}+45 \mathrm{pp}$.

GraY, G. (See Griffith \& Pidgeon, 1832)

Greene, C. T.

1923. A new species of Volucella (Diptera). Proc. Ent. Soc. Wash. 25: $165-168$.

1955. Larvae and pupae of the genera Microdon and Mixogaster (Diptera, Syrphidae). Trans. Amer. Ent. Soc. 81: 1-20.

GRIFFITH, E. \& Pidgeon, E.

1832. The class insects arranged by the Baron Cuvier, with supplementary additions to each order, and notices of new genera and species by George Gray, Esq. Volume the second. Vol. 15, 793 pp. (see Stone, et al., 1965:1251).

GUÉrin-MÉneville, F. E.

1844. Douzième ordre. Les Diptères. Pp. 531-559. In his Iconographie du règne animal de G. Cuvier, Vol. 3: Texte explicatif, Insectes, 576 pp. Paris (see Stone, et al., 1965:1253).

1848a. (Une description provisoire). Ann. Soc. ent. France (2) 6 (Bull.): lxxxi.

1848b. Note sur deux Insectes parasites de la cochenille et qui font un grand tort à cette culture en Amérique. Rev. zool. 11: $349-350$.

HALIDAY, A. H.

1856. Addenda and corrigenda. Pp. xi-xv. In (Walker, F., H. T. Stainton, \& S. J. Wilkinson), Insecta Britannica. (Vol. 4): Diptera Vol. 3 (by 'Walker), xxiv $+352 \mathrm{pp}$., pls. 21-30. London (see Stone, et al., 1965:1254, 1462).

HALL, D. G.

1927. A new Syrphid (Diptera) from Guatemala. Ent. News 38: 239-240.

HARDY, D. E.

1964. Diptera: Brachycera II-Cyclorrhapha I. Volume 11, $458 \mathrm{pp}$. in Zimmerman, E. C., Insects of Hawaii, volume 11. 
HARTLEY, J. C.

1961. A taxonomic account of the larvae of some British Syrphidae: Proc. Zool Soc. London 136(4): 505-573.

1963. The cephalopharyngeal apparatus of syrphid larvae and its relationship to other Diptera. Proc. Zool. Soc. London 141 (2) : 261-280.

HAYWARD, K. J. \& GOLBACH, R.

1963. Lista de los Tipos de Diptera conservados en el Instituto Miguel Lillo. Acta Zool. Lilloana: 19: 391-406.

HeISS, E. M.

1938. A classification of the larvae and puparia of the Syrphidae of Illinois exclusive of aquatic forms. Ill. Biol. Monog. 16 (4), $142 \mathrm{pp}$. in Univ. Ill. Bull. 36(1): 1-142.

HENDEL, F.

1908. Nouvelle classification des mouches à deux ailes (Diptera L.), d'après un plan tout nouveau par J. G. Meigen, Paris, an VII (1800 v. s.). Mit einem Kommentar. Verhandl. $k,-k$. zool.-bot. Gesell., Wien 58: 43-69.

HiNe, J. S.

1913. The genus Myiolepla [sic]. Ohio Nat. 14: 205-210.

1914. Diptera of Middle America. Family Syrphidae. Ohio Nat. 14: $333-343$.

1924. The North American species of the genus Meromacrus with one new species. (Diptera, Syrphidae), Bull. Brooklyn Ent. Soc. 19: 20-23.

HORN, W. \& KAHLE, I.

1935. Utber entomologische Sammlungen. (Ein Beitrag zur Geschichte der Entomo-Museologie). Teil 1. Ent. Beih., BerlinDahlem 2: 1-160, taf. i-xvi.

1936. Utber entomologische Sammlungen. (Ein Beitrag zur Geschichte der Entomo-Museologie). Teil II. Ent. Beih., BerlinDahlem 3:161-296, taf. xvii-xxvi.

1937. Über entomologische Sammlungen, Entomologen \& EntomoMuseologie. (Ein Beitrag zur Geschichte der Entomologie). Teil III. Ent. Beih. 4: 297-536, taf. xxvii-xxxviii.

HuLL, F. M.

1924. Milesia in North America (Dipt.: Syrphidae). Ent. News 35: $280-282$

1925a. A review of the genus Eritalis, pt. 1. Ohio J. Sci. 15: 11-43.

1925b. A review of the genus Eristalis, pt. 2. Ohio J. Sci. 15: 285-310. 
1925c. Notes on the North American species of the genus Didea with description of a new species. Ann. Ent. Soc. Amer. 18: $277-281$.

1930. Some new species of Syrphidae from North and South America. Trans. Amer. Ent. Soc. 56: 139-148.

1935. Some undescribed species of Eristalis from North America in the United States National Museum. J. Wash. Acad. Sci. 25: 326-331.

1936a. Change of same. Ent. News 4\%: 227.

1936b. A curious new Syrphid fly from Peru, Proc. Ent. Soc. Wash. 38: 167 .

1937a. Some Neotropical and Oriental Syrphid flies in the United States National Museum. J. Wash. Acad. Sci. 27: 165-176.

1937b. New species of exotic Syrphid flies. Psyche 44: 12-32.

1937c. A megamorphic and two curious mimetic flies. Psyche 44: 116-121.

1938. Exotic forms of Syrphid flies. Ann. Carnegie Mus. 27: 121-128.

1940a. Some Neotropical Syrphid flies. Ent. News 51: 247-250.

1940b. Some new species of Syrphidae. J. Wash. Acad. Sci, 30: $432-434$.

1941a. New American Syrphid flies. Amer. Mus. Novit. 1151: 1-3.

1941b. Some new species of Syrphidae. Bull. Brooklyn Ent. Soc. 36: 166-168.

1941c. Some new species of Syrphidae. J. Kansas Ent. Soc. 14: 61-63.

1941d. Some new Syrphid flies from North and South America. Ent. News 52: 157-163.

1941e. Some new species of Syrphidae from Florida, Cuba and Brazil. Ent. News 52: 278-283.

1941f. Some flies of the Family Syrphidae, Mesogramma. Psyche 48: $45-49$.

1941g. Descriptions of some new species of Syrphidae. Psyche 48: 149-165.

1941h. Some new species of Syrphidae from South America. J. Wash. Acad. Sci. 31: 311-315.

1941i. Some undescribed Syrphid flies from the Neotropical region. J. Wash. Acad. Sci, 31: 432-440. 
1941j. Some species of the genus Baccha from the New World. Proc: Ent. Soc. Wash. 43: 181-183.

1942a. New species of Syrphidae from the Neotropical region. Psyche 49: $84-107$.

1942b. Some new species of Syrphidae. J. Kansas Ent. Soc. 15: $10-12$.

1942c. Some new species of Baccha and Mesogramma. Ohio J. Sci. 42: 73-74.

1942d. Some new species of Baccha and Mesogramma. Rev. Ent., Rio de Janeiro 13: 44-49.

1942e. The flies of the genus Meromacrus. Amer. Mus. Novit. 1200: 1-19.

1942f. Some flies of the genus Mesogramma. Proc. New England Zool. Club. 30: 17-24.

1942g. Some flies of the genus Volucella. Proc. New England Zool. Club. 19: 93-98.

1942h. Some new species of Syrphidae. Psyche 49: 19-24.

1943a. New species of American Syrphid flies. Bull. Brooklyn Ent. Soc. 39: 48-53.

1943b. The genus Ceriogaster Williston (Syrphidae). Rev. Soc. Ent. Argentina 12: 138-140.

1943c. Two new species of Baccha (Syrphidae). Proc. Ent. Soc. Wash. 45: 50-51.

1943d. Some flies of the genus Volucella in the British Museum (Natural History). Ann. Mag. Nat. Hist. (2) 10: 18-40.

1943e. Some flies of the genus Microdon in the British Museum (Natural History). Ann. Mag. Nat. Hist. (2) 10: 702-720.

1943f. Some new American Syrphid flies (Diptera). Ent. News 54: 29-37.

1943g. New species of Syrphidae of the genus Baccha and Mesograma. Ent. News 54: 89-91.

1943h. New species of Baccha and related flies. Ent. News 54: 135-140.

1943i. The genus Mesogramma. Ent. Amer. 23: 1-41.

1943j. The New World species of the genus Baccha. Ent. Amer. 23: $42-99$. 
1943k. New species of Syrphid flies in the National Museum. $J$. Wash. Acad. Sci. 33: 39-43.

19431. Some undescribed species of the genus Baccha (Syrphidae). J. Wash. Acad. Sci. 33: 72-74.

1943m. New species of the genera Baccha and Rhinoprosopa (Syrphidae). J. Wash. Acad. Sci. 33: 214-216.

1943n. Some Neotropical Syrphids. Rev. Ent., Rio de Janeiro 14: 510-515.

1944a. New species of the genus Salpingogaster. Bol. Ent. Venezolana 3: 165-170.

1944b. Three new species of Syrphid flies in the British Museum of Natural History. Proc. Ent. Soc. Wash. 46: 10-12.

1944c. Studies on Syrphid flies in the Museum of Comparative Zoology. Psyche 51: 22-45.

1944d. Some flies of the family Syrphidae in the British Museum (Natural History). Ann. Mag. Nat. Hist., (11) 11: 21-61.

1944e. Additional species of Microdon in the British Museum (Natural History). Ann. Mag. Nat. Hist., (11) 11: 241-261.

1944f. New Syrphid flies from North and South America. Bull. Brooklyn Ent. Soc. 39: 35-40.

1944g. Additional species of the genus Baccha from the New World. Brooklyn Ent. Soc. Bull. 39: 56-64.

1944h. Some genera of flies of the family Syrphidae. J. Wash. Acad. Sci. 34: 129-134.

1944i. Studies on flower flies (Syrphidae) in the Vienna Museum of Natural History. J. Wash. Acad. Sci. 34: 398-404.

1944j. A study of some Syrphid flies of South America. Rev. Ent., Rio de Janeiro 15: 34-54.

1945a. New Syrphid flies. Ent. News 56: 210-217

1945b. Some undescribed Syrphid flies. Proc. New England Zool. Club. 23: 71-78.

1945c. A revisional study of the fossil Syrphidae. Bull. Mus. comp. Zool. 95: 251-355, pls. 1-13.

1946a. The genus Quichuana Knab. Amer. Mus. Novit. 1317: 1-17. 
1946b. The genus Lepidostola Mik. Amer. Mus. Novit. 1326: 1-15.

1947a. More flies of the genus Baccha (Diptera, Syrphidae). Rev. Ent., Rio de Janeiro 18: 395-410.

1947b. Some American Syrphid flies. Psyche 54: 230-240.

1948a. Some Neotropical species of Syrphids. Ent. News 59: 1-12.

1948b. Venezuelan Syrphid flies. Bol. Ent. Venezolana 7: 6-10.

1949a. American Syrphid flies of the subfamilies Cheilosinae and Syrphinae. Bull. Brooklyn Ent. Soc. 44: 73-79.

1949b. New species of New World Baccha. Ohio J. Sci. 49: 244-246.

1949c. Studies upon Diptera in the British Museum. Ann. Mag. Nat. Hist., (12) 2: 727-746.

1949d. The morphoolgy and inter-relationships of the genera of Syrphid flies, recent and fossil. Trans. Zool. Soc. London 26(4): $257-408$.

1949e. The genus Baccha from the New World. Ent. Amer. 27: 89-291 (1947).

1949f. Some flies of the genus Volucella from the New World. Psyche 56: 26-40.

1949g. New American Syrphid flies of the subfamily Eristalinae. Psyche 56: 120-138.

1949h. Some new species of the genus Volucella. Ent. News 60: 31-38.

1949i. Some unusual Syrphid flies. Ent. News 60: 225-233.

1950a. New species of New World Volucella. Bull. Brooklyn Ent. Soc. 45: 65-71.

1950b. Studies upon Syrphid flies in the British Museum (Natural History). Ann. Mag. Nat. Hist. (12) 3: 603-624.

1950c. New South American Syrphid flies. Rev. Ent., Rio de Janeiro 21: 225-236.

1951a. New species of Mesogramma (Diptera: Syrphidae). Amer. Mus. Novit. 1480: 1-22.

1951b. Some new species of Syrphid flies. J. New York Ent. Soc. 54: $65-70$.

1951c. Some New World Xylotinae (Diptrea: Syrphidae). PanPacific Ent. 27: 183-186. 
1954. The genus Mixogaster Macquart (Diptera: Syrphidae). Amer. Mus. Novit. 1652: 1-32.

1956. A new Southwestern species of Mallota Meigen (Diptera: Syrphidae). Psyche 63: 24-26.

1957. Some undescribed species of the genus Baccha Fabricius (Diptera: Syrphidae). J. New York Ent. Soc. 65: 187-190.

1958. Three new species of the genus Baccha Fabricius (Diptera: Syrphidae). Studia Ent. 1: 427-432.

1960. New species of Syrphidae and Asilidae (Diptera), Pan-Pac. Ent. 36: 69-74.

Hull, F. M. \& Fluke, C. L.

1950. The genus Cheilosia Meigen (Diptera, Syrphidae). The subgenera Cheilosia and Hiatomyia. Bull. Amer. Mus. Nat. Hist. 94: $299-402$.

INTERNATIONAL COMMISSION ON ZOOLOGICAL NOMENCLATURE.

1963. Opinion 678. The suppression under the Plenary Powers of the pamphet published by Meigen, 1800. Bull. zool. Nomencl. 20: $339-342$.

JACOBS, J. CH.

1900. Diagnoses d'Insectes recueillis par l'expedition Antarctique Belge. An. Soc. Ent. Belgique 44: 107.

JAENNICKE, F.

1867. Neue exotische Dipteren. Abhandl. Senckenb. Naturf. Gesell. 6: 311-408.

JOHNSON, C. W.

1894. List of the Diptera of Jamaica with descriptions of new species. Proc. Acad. Nat. Sci. Philadelphia, pp. 271-281.

1903. Diptera of Beulah, New Mexico. Pp. 101-106. In Skinner, H. ed., A list of the insects of Beulah, New Mexico. Trans. Amer. Ent. Soc. 29: 35-117.

1911. Notes on the dipterous genera proposed by Billberg in his Enumeratio Insectorum. Psyche 18: 73-74.

1924. Diptera of the Williams Galapagos Expedition. Zoologica 5 (8): 85-92.

1919. A revised list of the Diptera of Jamaica. Bull. Amer. Mus. Nat. Hist. 41(8): 421-449.

1908. The Diptera of the Bahamas with notes and description of one new species. Psyche 15: 69-80.

1919. The North American Diptera described by Nils S. Swederus. Canad. Ent. 51: 32 . 
1921. New Diptera from Texas and Mexico. Psyche 28: 56-59.

1926. New species of Diptera from North Carolina and Florida. Psyche (1925) 32: 299-302.

KAHL, P. H. I.

1897. New species of the syrphid genera Mixogaster Macq. and Ceria Fabr., with notes. Kans. Univ. Quart. (A) 6: 137-146.

KERTÉSZ, K.

1902. Uebersicht der südamerikanischen Ceria-Arten. Természetr. Füzetek 25: 85-90.

1903. Einige neue südamerikanische Ceria-Arten. Ann. Hist. Nat. Mus. hung. 1: 433-440.

1910. Catalogus dipterorum hucusque descriptorum Vol. 7, 470 pp.

KNAB，F.

1913. Some Neotropical Syrphidae. Insec. Inscit. Menst. 1: 13-15.

1914. Two North American Syrphidae. Insec. Inscit. Menst. 2: 151-153.

1916a. Critical notes on Syrphidae. Insec. Inscit. Menst. 4: 91-95.

1916b. Further notes on Syrphidae. Insec. Inscit. Menst. 4: 133-135.

1917. On sme North American Species of Microdon (Diptera: Syrphidae). Proc. Biol. Soc. Wash. 30: 133-143.

KNUTSON, L. V.

1971. Puparia of Salpingogaster conopida and S. texana, with notes on prey (Diptera: Syrphidae). Ent. News 82: 29-38.

1973. Taxonomic revision of the Aphid-killing flies of the genus Sphaerophoria in the Western Hemisphere (Syrphidae). Miscel. Publ. ent. Soc. Amer. 9: 1-50, 108 figs. 10 maps.

LANE, J.

1962. Insecta Patagonica (Diptera: Syrphidae). Rev. Soc. ent. Argentina 25: 17-19.

LANE, J. \& CARRERA, M.

1943. Sobre o gênero Cerioides (Diptera, Syrphidae, Cerioidini). Rev. Ent., Rio de Janeiro 14: 167-173.

1944. Duas espécies de Quichuana que se criam em bambu. Rev. Ent., Rio de Janeiro 15: 205-208.

Latreille, P. A., Lepeletier, A. L. M., Serville, J. G. A. \& Guérin-MéneVILLE, F. E.

1825-[1828]. Entomologie, ou histoire naturelle des Crustacés, de Arachnides et des Insectes Vol. 10, pp. 1-344, 1825; pp. 345-833 (1828) In Société de Gens de Lettres, de Savans et d'Artistes, Encyclopedie méthodique. Histoire naturelle. 
LATREIlle, P. A.

1804. Tableau méthodique des Insectes. Pp. 129-200. In Societé de Naturalistes et d'Agriculturs, Nouveau dictionnaire d'histoire naturelle, appliqué aux arts, principalement à l'agriculture et à l'économie rurale et domestique. Vol, 24: Tableaux méthodiques d'histoire naturelle, pp. 1-238.

1805. Histoire naturelle, générale et particulière, des Crustacés et des Insectes, Tome quatorzième. pp. 1-432. In Sonnini, C. S. ed., Histoire naturelle par Buffon.

1810. Considérations générales sur l’ordre naturel des animaux. 444 pp. Paris.

LATTA, R. \& COLE, F. R.

1933. A comparative study of the species of Eumerus known as the lesser bulb flies. Monthly Bull. Calif. Dept. Agric. 22: $142-152$, 16 figs.

LIMA, A. DA COSTA

1946. Nova espécie do gênero Rhysops Williston (Diptera: Syrphidae). Bol. Soc. brasil. Agron. 9: 155-156.

LiNNAEUS, C.

1758. Systema naturae per regna tria naturae. Ed. 10, vol. 1, 1-824. Stockholm.

LiNSLEY, E. G. \& R. L. USINGER

1966. Insects of the Galapagos Islands. Proc. Calif. Acad. Sci. (4) 33: 113-196 (168).

LOEW, H.

1840. Ueber die im Grosherzogthum Posen aufgefundenen Zweiflügler. Isis (Oken's) 1840: 512-583.

1853. Neue Beiträg zur Kenntnis der Dipteren. Erster Beitrag. $K$. Realschule Meseritz, Programme 1853 1-38.

1861. Diptera aliquot in insula Cuba collecta. Wien. Ent. Monatschr. 5: $33-43$.

1863. Diptera Americae septentrionalis indigena. Centuria tertia. Berlin. Ent. Zeitschr. 7: 1-55.

1864a. Diptera Americae septentrionalis indigena. Centuria quinta. Berlin. Ent. Zeitschr. 8: 49-104.

1864b. Diptera Americae septentrionalis indigena. Vol. 1: (Centuriae 1-5), 266 pp. Berolini (=Berlin), "1861" (see Stone, et al., $1965: 1325)$.

1866a. Diptera Americae septentrionalis indigena. Centuria sexta. Berlin. Ent. Zeitschr. (1865) 9: 127-186.

1866b. Diptera Americae septentrionalis indigena. Centuria septima. Berlin. Ent. Zeitschr. 10: 1-54.

1872a. Diptera Americae septentrionalis indigena. Centuria decima. Berlin. Ent. Zeitschr. 16: 49-115. 
1872b. Diptera Americae septentrionalis indigena. Vol. 2: (Centuria 6-10), 300 pp. Berolini (=Berlin), (see Stone, et al., 1965: 1326).

LIOY, $P$.

1864. I Ditteri distribuiti secondo un nuovo metodo di classificazione naturale. Atti $R$. Ist. veneto Sci. (3) 9: 738-760.

LYNCH ARRIBÁlZAGA, F.

1891. Dipterologia Argentina (Syrphidae). An. Soc. Cien. Argentina 32: 80-99; 118-131; 194-202; 247-256; 307-314.

1892a. Dipterologia Argentina (Syrphidae). An. Soc. Cien. Argentina 33: 51-58, 111-121; 189-199; 236-253.

1892b. Dipterologia Argentina (Syrphidae). An. Soc. Cien. Argentina 34: 33-46; 173-192; 242-280.

1892c. Dipterologia Argentina. Syrphidae. 181 pp., 1 pl. Buenos Aires.

Maldonado Capriles, J. \& Navarro, C. A.

1967. Additions and Corrections to Wolcott's "Insects of Puerto Rico." Carib. J. Sci. 7(1-2): 45-64 (59).

MACQUART, J.

1829. Insectes Diptères du nord de la France. Syrphies. Mém. Soc. Sci. Agr. Lille, 1827/1828: 149-371, 4 pls.

(also published separately as "Insectes Diptères du nord de la France”, Vol. 4, 223 pp., 4 pls., Lille, 1829).

1834. Histoire naturelle des Insectes. - Diptères, Tome premier. Diptera, Vol. 1, 578 pp., 12 pls. In [Roret, N. E., ed.] (Collection des suites $\grave{a}$ Buffon) Paris (see Stone et al., 1965: 1399).

1842. Diptères exotiques nouveaux ou peu connus. Mém. Soc. Sci. Agric. Lille 1841(1): 65-200, 22 pls.

(also published separately as "Diptères exotiques nouveaux ou peu connus", Vol. 2, Pt. 2, pp. 5-140, 22 pls., Paris, 1842) (see Stone et al., 1965:1332).

1846. Diptères exotiques nouveaux ou peu connus. Supplément. Mém. Soc. Sci. Agric. Lille (1845) 1844: 133-364, 20 pls.

(also published as "Diptères exotiques nouveaux ou peu connus. Supplément. pp. 5-238, 20 pls., Paris, 1846).

1847. Diptères exotiques nouveaux ou peu connus. 2e supplément. Mém. Soc. Sci. Agric. Lille 1846: 21-120, 6 pls.

(also published as "Diptères exotiques nouveaux ou peu connus. Supplément II", pp. 5-104, 6 pls., Paris, ?1847 (1848).

1848. Diptères exotiques nouveaux ou peu connus. Suite de $2^{\text {me }}$. Supplément [i.e., $3^{\text {e }}$ supplément]. Mém. Soc. Sci. Agric. Lille 1847 (2): 161-237, 7 pls. 
(also published as "Diptères exotiques nouveaux ou peu connus. Supplément III", pp. 1-77, 7 pls., Paris, 1848).

1850. Diptères exotiques nouveaux ou peu connus. $4 \mathrm{e}$ supplément. Mém. Soc. Sci. Agric. Lille 1849: 309-479, pls. 1-14.

(also published as "Diptères exotiques nouveaux ou peu connus. Supplément IV”, pp. 5-161 (text only), Paris, 1850).

1855. Diptères exotiques nouveaux ou peu connus. 5e supplément. Mém. Soc. Sci. Agric. Lille 1854: 25-156, 7 pls.

(also published as "Diptères exotiques nouveaux ou peu connus. Supplément V", pp. 5-136, 7 pls., Paris, 1855).

MANN, W. M.

1928. A new Microdon from Panama. Psyche 35: 168-170.

MÁRNEF, L.

1967. Austrosyritta cortesi nov. gen., nov. sp. de sírfido de Chile. (Diptera Syrphidae). Bull. Ann. Soc. R. Ent. Belg. 103: 268-276.

MaRTORELl, L. F.

1939. Insects observed in the State of Aragua, Venezuela, South America. J. Agric. Univ. Puerto Rico 23 (4): 177-232 (215-216).

Matsumura, S. \& J. Adachi

1917. Synopsis of the economic Syrphidae of Japan. Pt. II. Ent. Mag. (Kyoto) 2: 133-156, pl. 6.

MEIGEN, J. W.

1800. Nouvelle classification des mouches à deux ailes (Diptera L.) d'après un plan tout nouveau. $40 \mathrm{pp}$. Paris.

1803. Versuch einer neuen Gattungseintheilung der europäischen zweiflügeligen Insekten. Mag. Insektenkunde 2: 259-281.

1822. Systematische Beschreibung der bekannten europäischen zweiflügeligen Insekten. Vol. 3, pp. 1-416. Hamm.

1830. Systematische Beschreibung der bekannten europäischen zweiflügeligen Insekten. Vol. 6, pp. 1-401. Hamm.

MeIJeRE, J. C. H. DE

1913. Dipteren I. Nova Guinea 9: 305-386, taf. x.

Metcalf, C. L.

1921. The genitalia of male Syrphidae: their morphology, with especial reference to its taxonomic significance. Ann. Ent. Soc. Amer. 14(3): 169-214.

Mik, J. L.

1886. Dipterologische Miscellen. II. Wien. Ent. Ztg. 5: 276-279.

1895. Bemerkungen $\mathrm{zu}$ den Dipteren-Gattungen Pelecocera Meig. und Rhopalomera Wied. Wien. ent. Ztg. 14: 133-136. 
1899. Ueber die Dipterengattung Microdon. Wien. Ent. Ztg. 18: 138-143.

NEWMAN, E.

1838. Entomological Notes. Ent. Mag. (Newman) 5: 372-402.

Osten Sacken, C. R.

1875. A list of the North American Syrphidae. Bull. Buffalo Soc. Nat. Sci. 3: 38-71.

1878. Catalogue of the described Diptera of North America. Smithsn. Misc. Collect. 16(2): 1-276.

1877. Western Diptera: Descriptions of new genera and species of Diptera from the region west of the Mississippi and especially from California. Bull. U. S. Geol. Geog. Survey Ter., 3: $189-354$

Palisot de Beauvois, A. M. F. J.

1805-(1821). Insectes recueillis en Afrique et en Amérique dans les royaumes d'Oware et de Benin, à Saint-Domingue et dans les États-Unis, pendant les années 1786-1797. 276 pp. Paris. (see Stone, et al., 1965:1366).

PANZER, G. W. F.

1809. Faunae insectorum germanicae initiae oder Deutschlands Insecten. H. 104, 24 pp., 24 pls. Nürnberg. (see Stone, et al., 1965:1367).

PAPAVERO, N.

1962. Quatro novas espécies de Microdontinae do Brasil. (Diptera, Syrphidae). Papéis Avulso Zool. 15·(25): 317-326.

1964. Descrição de uma nova espécie de Ubristes Walker, 1852 (Diptera, Syrphidae, Microdontinae). Papéis Avulsos Zool. 16(2): 21-25.

Perty, J. A. M.

1830-1833. Delectus animalium articulatorum, quae in itinere per Brasiliam annis MDCCCXVII-MDCCCXX jussu et auspiciis Maximiliani Josephi I. Bavariae regis augustissimi peracto collegerunt Dr. J. B. de Spix et Dr. C. F. Ph. de Martius, $44+224$ pp., 40 pls. Monachii (=Munich). (Pp. 1-60, pls. 2-12, 1830; pp. $61-124$, pls. 13-24, 1832; pp. 125-224, pls. 25-40, 1833).

PHILIPPI, R. A.

1865. Aufzählung der chilenischen Dipteren. Verhandl $K$. $-k$. Zool. Bot. Gesell. Wien 15 (Abhandl.): 595-782.

PERSSON, P. I.

1971. "Eugenies resa". Localities, dates and labels of the insects collected during the voyage around the world by the Swedish frigate "Eugenie" in the years 1851-1853. Ent. Tidskr. 92: 164-172. 
PINC, G.

1962. Estudio de algunos caracteres morfológicos de nueve especies de Syrphidae chilenos de diferentes géneros (Diptera). Publ. Centros Estudios Entomologicos 4: 45-58.

Porter, Carlos, E.

1918. Artrópodos de Curacautin y Lonquimay. Rev. Chil. Hist. Nat. 22: 178-182.

1920. Sobre algunos Artrópodos colectados en diversas localidades del país por los señores J. N. Thomas, José A. Campo, J. A. Wolffsohn, R. Barros V., etc. Rev. Chil. Hist. Nat. 24: 153-160.

1921. Sobre algunos Sírfidos de Chile. Rev. Chil. Hist. Nat. 25: 446-447.

1923. Lista de los Sirfidos colectados por don Hector E. Pinochet C. en la prov. Bio-Bio Rev. Chil. Hist. Nat. 2\%: 117.

1924. Algunos Insectos colectados en el sur del país por el señor Flaminio Ruiz P. Rev. Chil. Hist. Nat. 28: 81-82.

1924. Enumeración de Sírfidos colectados en San José de Maipo en Octubre y Novbre. de 1923. Rev. Chil. Hist. Nat. 28: 98.

1927a. Sobre un Cerambycidae y un Syrphidae procedentes de Traiguen. Rev. Chil. Hist. Nat. 31: 83.

1927b. Cambio de nombre genérico en la Fam. Sirfidos, Rev. Chil. Hist. Nat. 31: 96.

1927c. Algunos insectos de Mancera. Rev. Chil. Hist. Nat. 31: 121-122.

1928. Entomología Chilena. Primera lista de Insectos de Panimavida. Rev. Chil. Hist. Nat. 32: 221-225.

1932. Acerca de algunos Insectos Chilenos. Rev. Chil. Hist. Nat. 36: 190-193.

1934. Insectos colectados en Puente Alto (1). Rev. Chil. Hist. Nat. 38: 169-171.

1937. Acerca de la distribucion geográfica de algunos Insectos Chilenos. Rev. Chil. Hist. Nat. 41: 41-42.

1938. Entomologia Chilena. Localidades Nuevas de algunas especies. Rev. Chil. Hist. Nat. 42: 166-169.

RAFINESQUE, C. S.

1815. Analyse de la Nature ou tableau de l'Univers et des corps organisés. 224 pp. Palerme. 
RAMOS, J. A.

1946. The Insects of Mona Island (West Indies). J. Agric. Univ. Puerto Rico 30(1): 1-74 (57).

RÖDER, V. vON

1885. Diptera von der Insel Portorico. Stettin. Ent. Ztg. 46: 337-349.

1886. Dipteren von den Cordilleren Colombien. Gesammelt durch Herrn Dr. Alphons Stübel. Stettin. Ent. Ztg. 47: 257-270.

1892. Dipteren gesammelt in den Jahren 1868-1877 auf einer Reise durch Süd-Amerika von Alphons Stübel. Berlin. $16 \mathrm{pp}$.

RONDANI, C.

1844. Proposta della formazione di un genere nuovo per due specie di insetti ditteri. Memoria nona per servir alla ditterologia italiana. Nuovi Ann. Sci Nato, Bologna (2) 1 [sic; = 2]: 193-202, pl. 2

1845. Ordinamento sistematico dei generi italiani degli insetti ditteri. Nouvi Ann. Sci. nat. Bologna (2) 2: 256-270, 443-459.

1848. Esame di varie specie d'insetti ditteri brasiliani. Studi Ent. (Turin) 1: 63-112

1850a. Osservazioni sopra alquante specie di esapodi ditteri del Museo Torinese. Nuovi. Ann. Sci. Nat. Bologna, (3) 2: 165-197.

1850b. Dipterorum species aliquae in America Aequatoriali Collectae a Cajetano Osculati observatae et distinctae novis breviter descriptis. Nuovi Ann. Sci. Nat. Bologna (3) 2: 355-373.

1850c. Nota sexta pro dipterologia italica de nova specie generis Ceriae Fabricii. Ann. Soc. ent. France (2) 8: 211-214, pl, 7, fig. 1 .

1857. Dipterologiae Italicae prodomus. Vol. 2: Species Italicae ordinis dipterorum in genera characteribus definito, ordinatim collectae, methodo analitica distinctae, et novis vel minus cognitis descriptis, Pars Prima: Oestridae, Syrpfhidae (sic), Conopidae. 264 pp. Parma.

1863. Diptera exotica revisa et annotata. 99,pp., 1 pl. Modena.

(Also published as "Dipterorum species et genera aliqua exotica", 1864, Arch. Zool. anat. Fis., Modena (1863) 3(1): 1-99, pl. 5).

1868. Diptera aliqua in America meridionali lecta a Prof. P. Strobel annis 1866-67. Annuar. Soc. Nat. Modena 3: 24-40, pl. 4.

SAChtleBen, H.

1961. Nachträge zu "Walther Horn \& Ilse Kahle: Über entomologische Sammlungen". Beitr. Ent. 11: 481-540. 
SACK, P.

1920. Die Gattungen Salpingogaster Schiner und Meromacrus Rondani. Zool. Jahrb., Abt. System., Geog. Biol. Tiere 43: 235-272.

1921. Dr. L. Zürchers Dipteren-Ausbeute aus Paraguay: Syrphiden. Archiv Naturgesch. (Abt. A), 87: 127-149.

1941. Syrphidae, pp. 97-120. In Titschack, E, Beiträg zur Fauna Perus. Band $I, 344 \mathrm{pp}$.

(most copies of this publication were destroyed during World War II. It was re-issued as Sack, 1951. see Zool. Rec. 1951, 88. (13): 132 [\#2537]).

1951. Syrphidae, pp. 93-116. In Titschack, E., Beiträge zur Fauna Perus. Band II, $344 \mathrm{pp}$.

SAY, T.

1823. Descriptions of dipterous insects of the United States. $J$. Acad, Nat. Sci. Phila. 3: 73-104.

1829-1830. Descriptions of North American dipterous insects. J. Acad. Nat. Sci Phila. 6: 149-178, 1829; 183-188, 1830.

SCHINER, I. R.

1860. Vorläufiger Commentar zum dipterologischen Theile der "Fauna Austriaca". II. Wien. Ent. Monatschr. 4: 208-216.

1868. Diptera. In Reise der österreichischen Fregatte Novara Zool., Vol. 2, Abt. 1, B. Wien. 388 pp. (see Stone, et al., 1965:1480).

SCOPOLI, J. A.

1763. Entomologia carniolica exhibens insecta carnioliae indigine et distributa in ordines, genera, species, varietates methodo Linnaeana. 421 pp. Vindobonae [=Vienna].

SEDMAN, Y. S.

1959. Male genitalia in the subfamily Cheilosinae. Genus Chrysogaster s. 1. (Diptera, Syrphidae). Proc. ent. Soc. Wash. $61(2): 49-58$.

1964. The Chrysogaster (Orthonevra) bellula group in North America. Proc. Ent. Soc. Wash. 66: 169-201.

1965. The Genus Valdivia Shannon. Proc. Ent. Soc. Wash. 67: 197-201.

SHANNON, R. C.

1921. A reclassification of the subfamilies and genera of the North American Syrphidae, Bull. Brooklyn Ent. Soc. 16: 65-72.

1922a. A reclassification of the subfamilies and genera of the North American Syrphidae. Bull. Brooklyn Ent. Soc. (1921) 16: 120-128. 
1922b. A reclassification of the subfamilies and genera of the North American Syrphidae. Bull. Brooklyn Ent. Soc. 17: 30-43.

1923a. A reclassification of the subfamilies and genera of the North American Syrphidae (Diptera). Appendix. Bull. Brooklyn Ent. Soc. 18: 17-21.

1923b. A new Microdon from Bolivia. Insec. Inscit. Menst. 11:80-81.

1925a. Some American Syrphidae (Diptera). Proc. Ent. Soc. Wash. 27: $107-112$.

1925b. An extraordinary adult myrmecophile from Panama, J. Wash. Acad. Sci. 15: 211-213.

1925c. The syrphid-flies of the subfamily Ceriodinae in the U.S. National Museum Collection. Insec. Inscit. Menst. 13: 48-52, 53-65.

1926a. Review of the American xylotine syrphide-flies. Proc. U. S. nat. Mus. 69(9): 1-52.

1926b. The chrysotoxine syrphid-flies, Proc, U. S. nat. Mus. 69(11): 1-20.

1927a. A review of the South American two-winged flies of the family Syrphidae. Proc. U. S. Nat. Mus $70(9): 1-33$.

1927b. Notes on and descriptions of syrphid flies of the subfamily Cerioidinae. J. Wash. Acad. Sci. 17: 38-53.

1927c. Una nueva especie de Syrphidae: Quichuana Rieseli. Rev. Soc. Ent. Argentina 1: 5-6.

1927d. Some New Diptera from Argentina. Rev. Soc. Ent. Argentina 1: $31-42$.

1928a. Two new and remarkable Syrphidae in the Museo Nacional de Historia Natural de Buenos Aires. An. Mus. Nac. Hist. Nat., Buenos Aires 34: 573-576.

1928b. Apropos "Masarygus" Brèthes. Rev. Soc. Ent. Argentina 2: $37-38$.

Shannon, R. C. \& Aubertin, D.

1933. Syrphidae. Diptera of Patagonia and South Chile. Brit. Mus. (Nat. Hist.) part VI, fascicle 3: 120-170. (see Stone, et al., 1965:1163).

Sмiтн, F.

1877. Account of the Zoological Collections made during the visit of H. M. S. "Petrel" to the Galapagos Islands. VIII. Hymenoptera and Diptera. Proc. Zool. Soc. London 45: 82-84.

SNow, W. A.

1895a. A new species of Pelecocera. Kansas Univ. Quart. 3: 187. 
1895b. Diptera of Colorado and New Mexico. Syrphidae. Kansas Univ. Quart. 3(4): 225-247.

Soukup, J.

1945. Lista de los Dípteros peruanos descritos en los ultimos años. Bol. Mus. Hist. nat. "Javier Prado" 9: 119-142 (128-129).

1959. Segunda lista de Dípteros del Peru. Biota 2: 178-195, 201-223.

SPEISER, P.

1914. Ueber einige Syrphiden und zwei für die deutsche Fauna neue Clythiiden. Jahrb. Naturk., Wiesbaden (1913) 66: 117-146.

STEPHENS, J. F.

1846. Illustrations of British Entomology; or, a Synopsis of indigenous insects: containing their generic and specific distinctions; with an account of their metamorphoses, times of appearance, localities, food, and economy, as far as practicable. Supplement. vi +32 pp. London.

STEYSKaL, G. C.

1953. A new melissomimetic fly of the genus Microdon (Diptera Syrphidae). Occ. Pap. Mus. Zool. Univ. Michigan 551: 1-4.

Stone, A., C. Sabrosky, W. W. Wirth, R. H. Foote, \& J. Coulson

1965. A catalog of the Diptera of America north of Mexico. U. S. Dept. Agric. Handb. 276, $1696 \mathrm{pp}$.

Stuardo Ortiz, Carlos

1946. Catálogo de los Dipteros de Chile. 251 pp. Min. Agric. [Chile], Santiago.

STUARDO, C. \& R. CORTÉS

1952. Nuevo nombre para un género de Syrphidae Chileno de R. A. Philippi (Diptera). Revta. Chil. Ent. 2: 311.

SZILÁDY, Z.

1926. Dipterenstudien. Ann. Hist. nat. Mus. hung. 24: 586-611, 18 figs.

THOMPSON, F. CHRISTIAN

1965. A New Neotropical Lepidosis Curran (Diptera: Syrphidae). Ent. News 76: 61-62.

1968. The Placement of the Subgenus Protolepidostola Hull (Diptera: Syrphidae) with the description of two new species. J. Kans. Ent. Soc. 41: 270-277.

1969. A New Genus of Microdontine Flies (Diptera: Syrphidae) with Notes on the Placement of the Subfamily. Psyche 76 : 74-85.

1971. The genus Nepenthosyrphus with a key to world genera of Tropidiini. J. Kansas Ent. Soc, 44: 523-534. 
1972. A contribution to a generic revision of the Neotropical Milesinae (Diptera: Syrphidae). Arq. Zool. 23: 73-215, 74 figs., 11 maps.

THOMson, C. G.

1869. 6. Diptera, Species nova descripsit. Pp. 443-614, In K. Svenska Vetenskaps-Akademien, Kongliga svenska fregatten Eugenies resa omkring jorden. Pt. Zoologie, 1: Insekter, 617 pp. (see Stone et al., 1965:1297, 1442).

TOWNSEND, C. H. T.

1895a. Contributions to the Dipterology of North America. I. Syrphidae. Trans. Amer. Ent. Soc. 22: 33-55.

1895b. On the Diptera of Baja California, including some species from adjacent regions. Proc. Calif. Acad. Sci. (2) 4: 593-620.

1897a. Contributions from the New Mexico Biological Station. No. 2. On a collection of Diptera from the Lowlands of the Rio Nautla, in the State of Vera Cruz. I. Ann. Mag. Nat. Hist. (6) $19: 16-34$.

1897b. Contributions from the New Mexico Biological Station. No II (continued). On a collection of Diptera from the lowlands of the Rio Nautla, in the State of Vera Cruz. II. Ann. Mag. Nat. Hist. (6) 20: 19-33.

1897e. Diptera from the lower Rio Grande or Tamaulipan Region of Texas. I. J. N. Y. Ent. Soc. 5: 171-178.

1897d. Diptera from the headwaters of the Gila River. I. Psyche 8: 38-41.

1897e. Contributions from the New Mexico Biological Station. No IV. Diptera from the Sacramento and the White Mountains in southern New Mexico. I. Ann. Mag. nat. Hist. (6) 19: 138-149.

1898. Diptera from the Lower Rio Grande or Tamaulipan Fauna of Texas. II. J. New York ent. Soc. 6: 50-52:

1901. Diptera from the headwaters of the Gila River. II. Psyche 8: $92-94$.

TUCKER, R. W. E.

1952. The Insects of Barbados. J. Agric. Univ. Puerto Rico 36: 330-363 (349).

1953. Insects of Barbados. J. Barbados Mus. hist. Soc. 20: 155-181 (172).

VAL, F. C. DO

1972. On the biometry and evolution of the genus Ornidia (Diptera, Syrphidae). Papéis Avulsos Zool. 26(1): 1-28, 5 figs., 9 graphs.

VERRALL, G. H.

1901. British flies. Vol. 8, Platypezidae, Pipunculidae, and Syrphidae of Great Britain, 691 pp. London (see Stone et al., 1965:1458). 
1913. Descriptions of new species of the surphid genus Callicera. Trans, Ent. Soc. London, (3) 1913: 323-333.

VIMMER, A. \& J. Soukup

1938. Los Dipteros de los alrededores de Puno. Bol. Mus. Hist. nat. "Javier Prado" 2: 27-35.

VOCKEROTH, J. R.

1964. The Genus Alipumilio Shannon (Diptera: Syrphidae). Canad. Ent. 96: 922-924.

1969. A Revision of the Genera of the Syrphini (Diptera: Syrphidae). Mem. Canad. Ento. Soc, 62: 1-176.

WALKER, F.

1837. Descriptions, etc. of the Diptera. Pp. 331-359. In Descriptions, etc. of the Insects collected by Captain P. P. King, R. N., F. R. S., in the Survey of the Straits of Magellan. In Curtis, J., Haliday, A. H. and Walker, F., Trans, Linn. Soc. London 17: $315-359$.

1849. List of the specimens of dipterous insects in the collection of the British Museum. Vol. 3, 485-687. London.

1852. Diptera cont. Vol. 1, pp. 157-414. In [Saunders, W. W., ed.J. Insecta Saundersiana, London, "1856" (see Stone et al., 1965:1461).

1857. Characters of undescribed Diptera in the collection of W. W. Saunders, Esq., F. L. S., etc. Trans. Ent. Soc. London 4: 119-158.

1860. Characters of undescribed Diptera in the collection of W. W. Saunders. Trans. Ent. Soc. London 5: 268-296.

WESTWOOD, J. O.

1840. Order XIII. Diptera Aristotle (Antliata Fabricius. Halteriptera Clairv.). pp. 125-158. In his, An introduction to the modern classification of insects. Synopsis of the genera of British insects, 158 pp. London. (see Stone et al., 1965:1466).

WHEELER, W. M.

1924. Two extraordinary larval myrmecophiles from Panama. Proc. Nat. Acad. Sci. 10: 237-244.

WIEDEMANN, C. R. W.

1819. Brasilianische Zweiflügler. Zool. Mag. (Wied.) 1(3): 40-56.

1824. Munus rectoris Academia Christiana Albertina aditurus analecta entomologica ex Museo Regio Havniensi. 60 pp. Kiliae $[=$ Kiel $]$.

1830. Aussereuropäische zweiflügelige Insekten. Vol. 2, 684 pp. Hamm. 
Williston, S. W.

1882a. New or little known genera of North American Syrphidae. Canad. Ent. 14: 77-80.

$1882 \mathrm{~b}$. Contribution to a monograph of the North American Syrphidae. Proc. Amer. Phil. Soc. 20: 299-332.

1883. On the North American Asilidae (Dasyponinae, Laphrinae), with a new genus of Syrphidae. Trans. Amer. Ent. Soc. 11: $1-35$.

1885. On the classification of North American Diptera. (First Paper). Bull. Brooklyn ent. Soc. 7: 129-139.

1886. Catalogue of the described species of South American Syrphidae. Trans. Amer. Ent. Soc. 14: 308-324.

1887a. Catalogue of the described species of South American Syrphidae. Additions and Corrections. Ent. Amer. 3: 27-28.

1887b. Synopsis of the North American Syrphidae. Bull. U.S. Nat. Mus. (1886) 31: 1-335.

1888. Diptera Brasiliana, ab H. H. Smith Collecta. Trans. Amer. Ent. Soc. 15: 243-292.

1891. Fam. Syrphidae. Pp. 1-56. In Godman, F. D., and Salvin, O., ed., Biologia Centrali-Americana. Zoologia-Insecta-Diptera, vol. 3, $127 \mathrm{pp}$. (see Stone, et al., 1965:1245).

1892. Fam. Syrphidae. Pp. 57-79. In Godman, F. D., and Salvin, O., eds., Biologia Centrali-Americana. Zoologia-Insecta-Deptera, vol. 3, $127 \mathrm{pp}$. (see Stone et al., 1965:1245):

1896. Manual of the families and genera of North American Diptera. Ed. 2. 167 pp. New Haven, Conn.

1907. Dipterological notes. J. N. Y. Ent. Soc. 15: 1-2.

Wirth, W. W., Y. S. Sedman \& H. V. WeEms, JR.

1965. Family Syrphidae. In: Stone, A., C. Sabrosky, W. W. Wirth, R. H. Foote \& J. Coulson. 1965. A catalog of the Diptera of America north of Mexico. U.S. Dept. Agric. Handb. 276, $1696 \mathrm{pp}$.

WoLCOTT, G. N.

1923. "Insectae Portoricensis". A Preliminary annoted check-list of the insects of Puerto Rico, with descriptions of some new species. J. Dept. Agric. Puerto Rico \%(1): 1-312 (217-220).

1927. Entomologie d'Haiti. 440 pp., 133 figs. Port-au-Prince (319321).

1936. "Insectae Borinquenses". A revised annotated check-list of the insects of Puerto Rico. J. Agric. Univ. Puerto Rico 20 (1): $627+3$ pp., illustr. (346-352). 
1941. Supplement to "Insectae Borinquenses". J. Agric. Univ. Puerto Rico 25(2): 33-158 (115).

1948. The Insects of Puerto Rico. Diptera. J. Agric. Univ. Puerto Rico 32(3): 417-532, figs. (461-469).

1971. Los Insectos. In Cendero, L., Zoologia Hispanoamericana, pp. 567-898. (749-752).

WULP, F. M. VAN DER

1867. Eenige Noord-Americaansche Diptera. Tijdschr. Ent. 10: 125-164.

1882. Amerikaansche Diptera. Tijdschr. Ent. 25: 77-136.

1883. Amerikaansche Diptera. Tijdschr, Ent. 26: 1-60.

1888. Nieuwe Argentijnsche Diptera van wijlen Prof. H. Veyenbergh. Tijdschr. Ent. 31: 359-376.

1891. Eenige uitlandische Diptera. Tijdschr. Ent. (1890) 34: 193218.

ZetTerstedT, J. W.

1838. Dipterologis Scandinaviae. In his Insecta Lapponica. Sec. 3: Diptera, pp. 447-868.

ZIMSEN, E.

1964. The type material of I. C. Fabricius. 656 pp. Copenhagen.

\section{INDEX}

abata (Curran), 1938 (Baccha), Ocyptamus ............ 46.11

abdominale (Wiedemann), 1830 (Volucella), Copestylum ..... 46.70

abdominalis Sack, 1920, Meromacrus ................. 46.111

abdominalis Sack, 1920, Salpingogaster .............. 46.31

abdominalis (Williston), 1888 (Lepidostola), Lepidomyia .... 46.88

aberrans Shannon, 1927, Mallota ................. 46.99

abnormis Curran, 1925, Microdon ................. 46.62

abrupta (Curran), 1925 (Phalacromyia), Copestylum ....... 46.70

abrupta Hine, 1914, Dolichogyna ................. 46.97

acra (Curran), 1941 (Cerioides), Sphiximorpha ........... 46.93

Acrochordonodes Bigot, $1878 \ldots \ldots \ldots \ldots \ldots \ldots \ldots \ldots \ldots \ldots . \ldots \ldots . \ldots \ldots$

acutifrons (Curran), 1939 (Volucella), Copestylum ......... 46.71

acutus (Fabricius), 1805 (Milesia), Meromacrus ........... 46.111

ada (Curran), 1941 (Baccha), Ocyptamus ............... 46.12

adspersus (Fabricius), 1805 (Baccha), Ocyptamus .......... 46.12

a $\in$ mula (Williston), 1888 (Volucella), Ornidia ............. 46.69

aemula (Williston), 1891 (Eristalis), Palpada ............. 46.102

áemulus (Williston), 1888 (Pteroptila), Meromacrus ........ 46.111

aenea (Hull), 1937 (Oligorhina), Allograpta ............ 46.33

aeneus (Williston), 1891 (Baccha), Ocyptamus .......... 46.12 


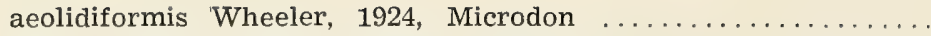

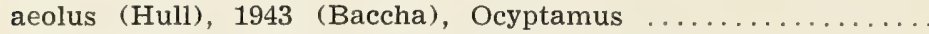

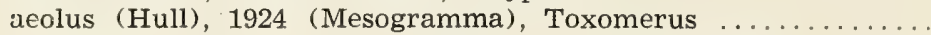
aequilineatus (Hull), 1945 (Epistrophe), Ocyptamus aeruginosifrons (Schiner), 1868 (Syrphus), Allograpta ........

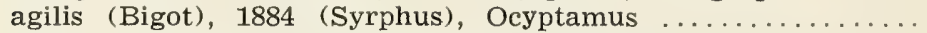

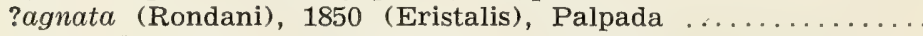
agonis (Walker), 1849 (Syrphus), Rhysops

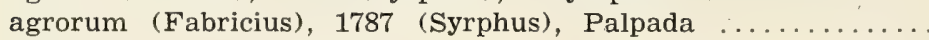

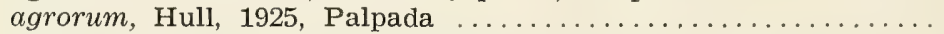

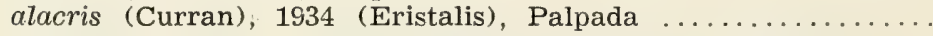
albiceps (Macquart), 1846 (Helophilus), Polydontomyia ....... albifrons (Curran), 1939 (Volucella), Copestylum ............

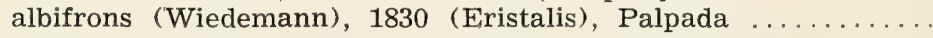
albimanus (Bigot), 1883 (Ocyptamus), Valdiviomyia albipilosum (Hull), 1937 (Volucella), Copestylum ............ albitarse (Lynch Arribálzaga), 1892 (Phalacromya), Copestylum

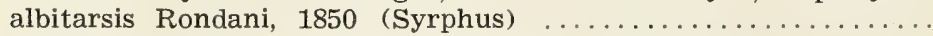

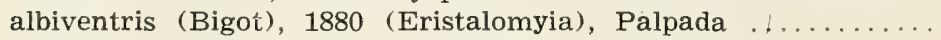
albomaculatus (Smith), 1877 (Syrphus), Xanthandrus ........

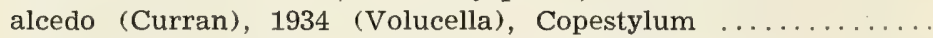
alcedoides (Curran), 1939 (Volucella), Copestylum alchimista (Rondani), 1848 (Volucella), Copestylum ..........

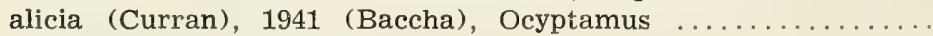

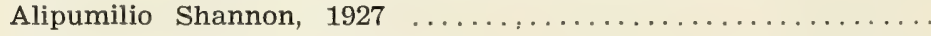

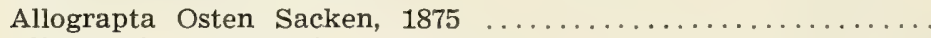

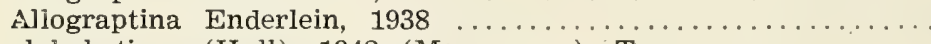

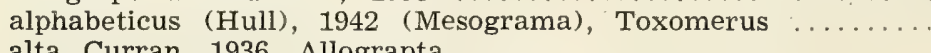

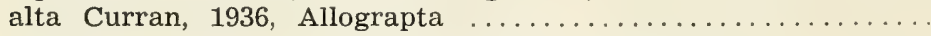
altissima (Fluke), 1942 (Epistrophe), Allograpta . ...........

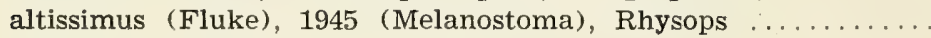

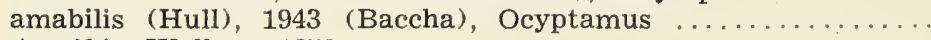
Amathia Walker, 1852

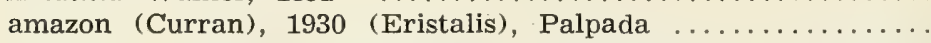
ambrosettii (Lynch Arribálzaga), 1892 (Volucella), Copestylum

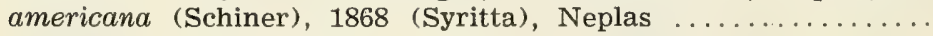
americanum (Goot), 1964 (Volucella), Copestylum .......... americanus (Wiedemann), 1830 (Syrphus), Metasyrphus ..... amethystinum (Bigot), 1875 (Volucella), Copestylum ........

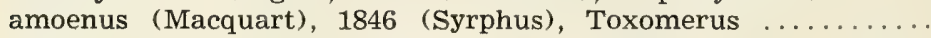

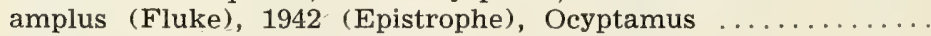

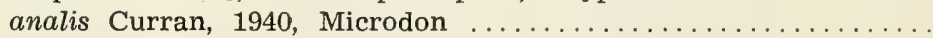

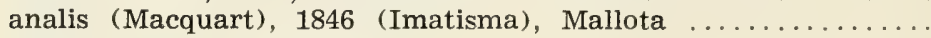

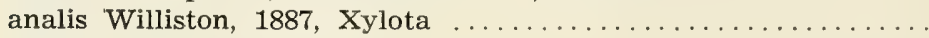

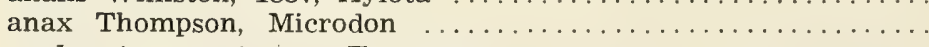

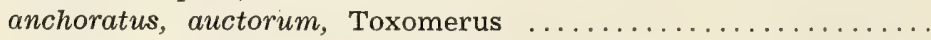

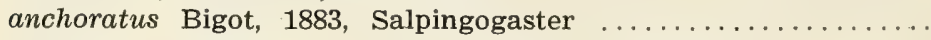

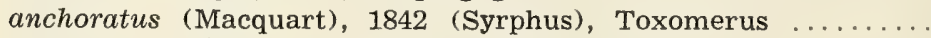
andicolum (Bigot), 1884 (Temnocera), Copestylum ..........

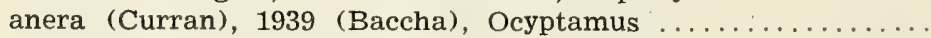
Aneriophora Stuardo \& Cortés, $1952 \ldots \ldots \ldots \ldots \ldots \ldots \ldots$

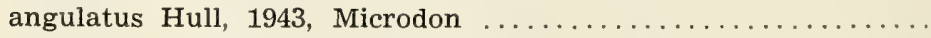
?angustata (Rondani), 1848 (Eristalis), Palpada ........... angustatus, Fluke, 1957, Microdon 
angustiventris (Macquart), 1855 (Aphritis), Microdon ....... angustiventris (Macquart), 1855 (Merodon), Quichuana .....

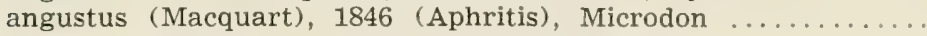

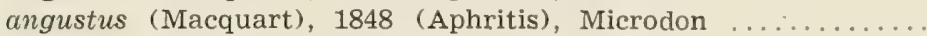

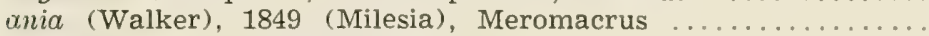

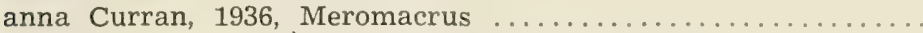
anna (Williston), 1887 (Volucella), Copestylum annulatus (Curran), 1941 (Callostigma), Ocyptamus ......... annulifer (Bigot), 1884 (Melanostoma), Toxomerus .......... annulifera Bigot, 1884, Orthonevra

annulipes (Macquart), 1850 (Paragus) Allograpta ...........

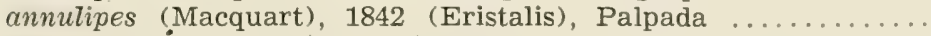

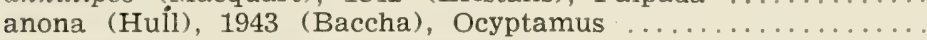
antennalis Philippi, 1865, Sterphus antennatum Fluke, 1943, Tuberculanostoma

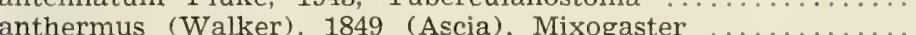
anthinone (Hull), 1949 (Baccha), Ocyptamus

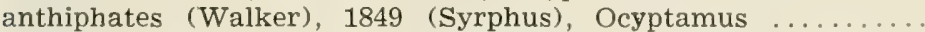
anthracoides (Bigot), 1884 (Melanostoma), Ocyptamus ........ anthrax (Schiner), 1868 (Mesogramma), Toxomerus ........

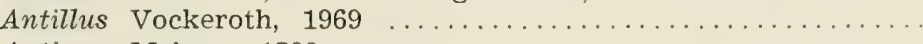
Antiopa Meigen, 1800

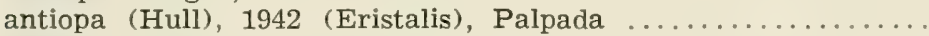

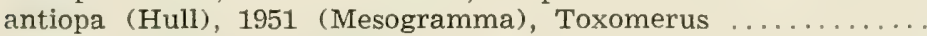

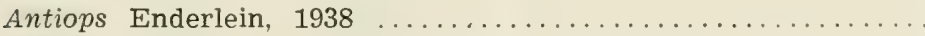

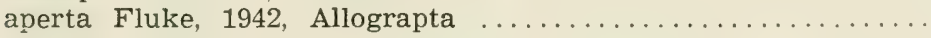
apertum (Walker), 1860 (Volucella), Copestylum ...........

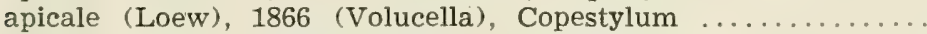
apicalis (Bigot), 1883 (Baccha), Leucopodella ...............

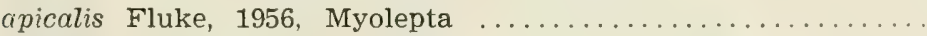
apiciferum (Townsend), 1895 (Volucella), Copestylum ........ apicula (Curran), 1939 (Volucella), Copestylum .............

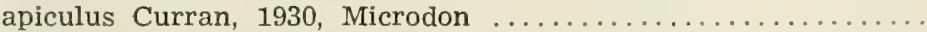

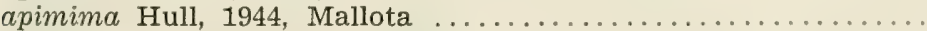

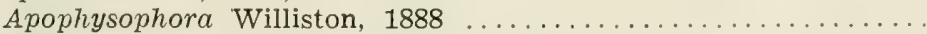

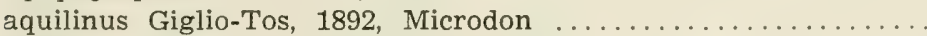

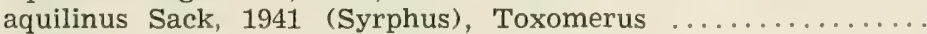

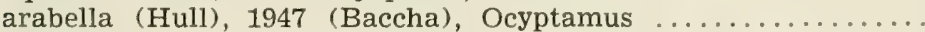

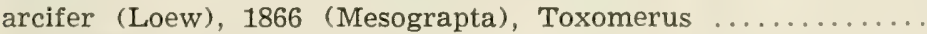

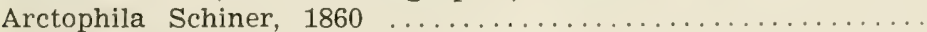
arctophiloides (Giglio-Tos), 1892 (Crioprora), Criorhina ..... arcturus (Hull), 1943 (Mesogramma), Toxomerus ...........

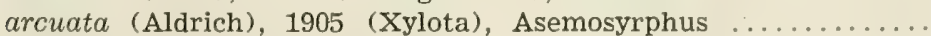

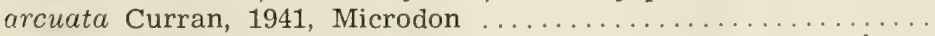

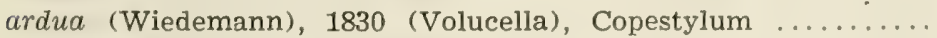

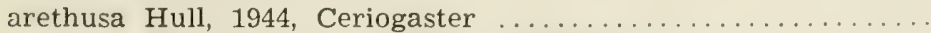

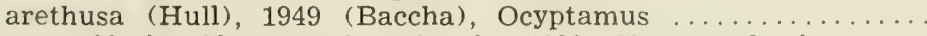
argentifacies Shannon \& Aubertin, 1933, Chamaesphegina .... argentina (Bigot), 1883 (Phalacromyia), Copestylum ......... argentina (Brèthes), 1922 (Chrysogaster), Orthonevra ........ argentinae Hull, 1937, Microdon 
argyropila (Hull), 1938 (Eristalis), Palfada .............. arica (Fluke), 1950 (Volucella), Copestylum $\ldots \ldots \ldots \ldots \ldots \ldots$

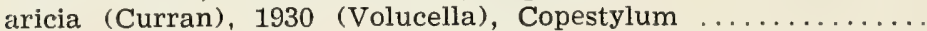

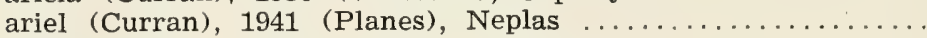

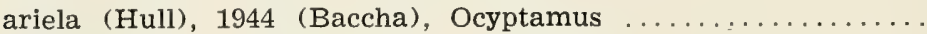

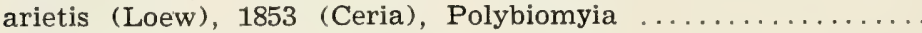

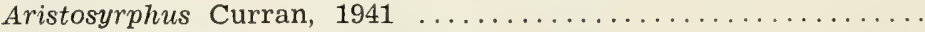

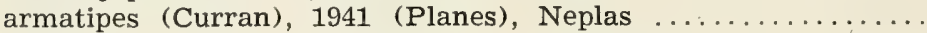

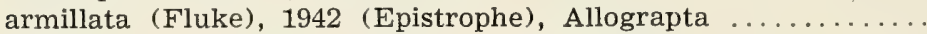

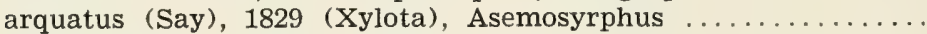

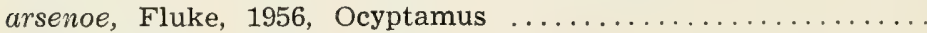

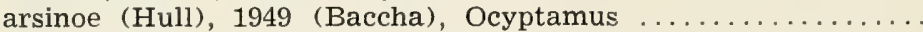

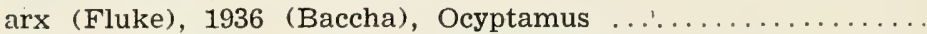
ascita (Vockeroth), 1969 (Antillus), Allograpta Asemosyrphus Bigot, 1882

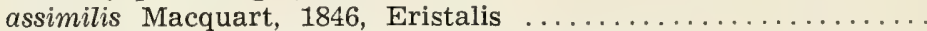

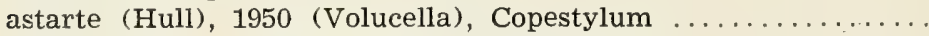

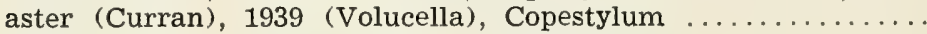

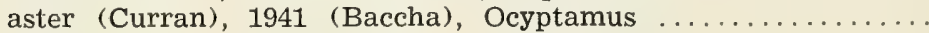

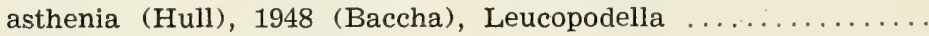

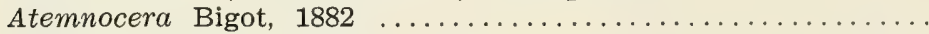

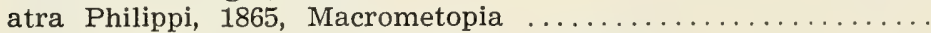

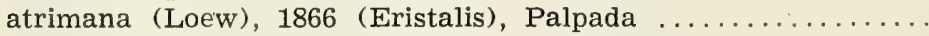

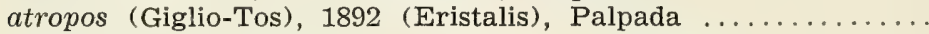

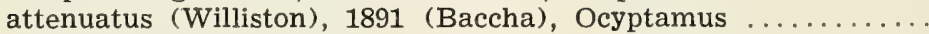
Atylobaccha Hull, 1949

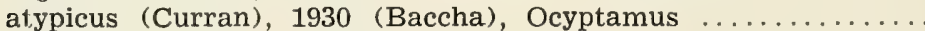

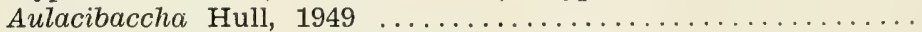

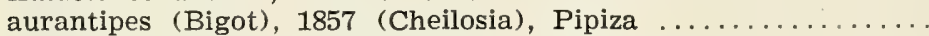

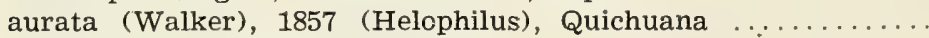

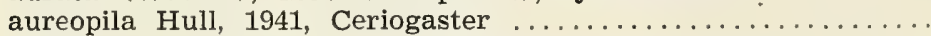
aureorufa (Philippi), 1865 (Eriophora), Aneriophora ..........

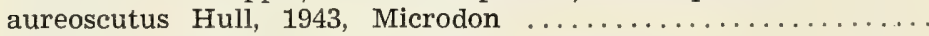
aureum (Fluke), 1951 (Volucella), Copestylum .............

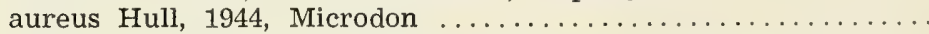

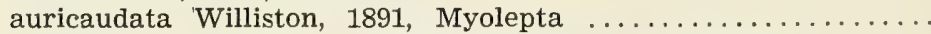

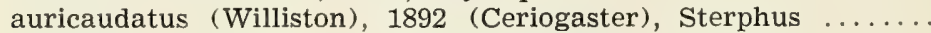
auricinctus (Sack), 1921 (Rhopalosyrphus), Microdon ..........

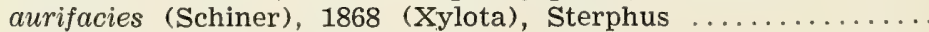

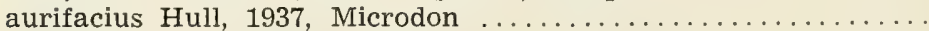

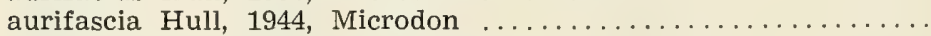

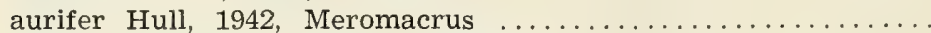

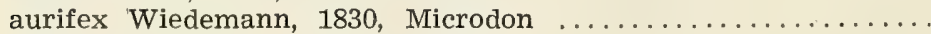

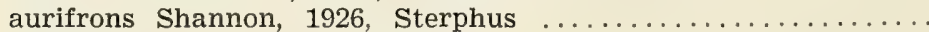

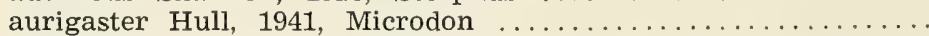

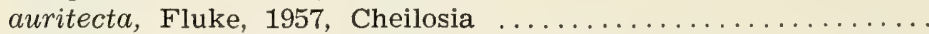

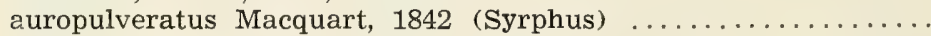

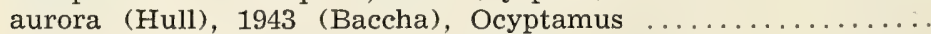

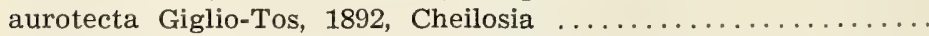

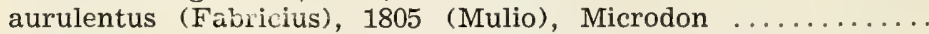
aurulentus (Williston), 1887 (Mesograpta), Toxomerus ........

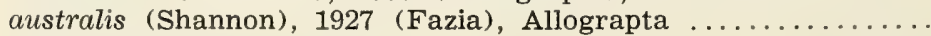

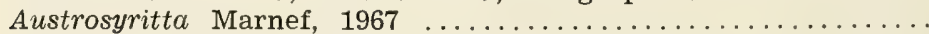

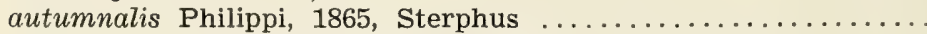
avidum (Oosten Sacken), 1877 (Volucella), Copestylum ......

46.103

46.71

46.71

46.114

46.13

46.95

46.57

46.114

46.34

46.96

46.27

46.27

46.13

46.34

46.96

46.102

46.71

46.71

46.13

46.46

46.70

46.116

46.103

46.110

46.13

46.11

46.13

46.11

46.67

46.98

46.115

46.118

46.63

46.71

46.63

46.89

46.117

46.63

46.116

46.63

46.63

46.111

46.63

46.116

46.63

46.68

46.39

46.13

46.68

46.63

46.48

46.36

46.119

46.116

46.72 
avispas Vockeroth, 1964, Alipumilio

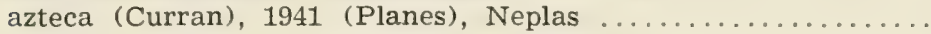

46.114

aztecarum Wheeler, 1924, Nothomicrodon

46.67

aztecus Hull, 1935, Eristalis

46.101

azurea (Fluke), 1953 (Heliophilus), Xylota

46.114

azureum (Philippi), 1865 (Volucella), Copestylum

46. 72

azurinum (Hull), 1941 (Volucella), Copestylum .............

46.72

azurlineus (Hull), 1941 (Mesogramma), Toxomerus

46.48

Baccha, authors

46.11

bacchiformis (Vockeroth), 1969 (Hermesomyia), Ocyptamus ...

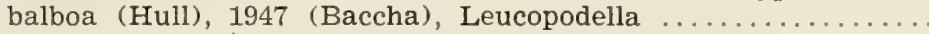

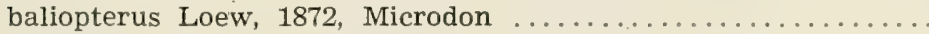

banksi Hull, 1942, Microdon

46.25

46.46

46.63

46.63

banksi (Hull), 1941 (Baccha), Ocyptamus

46.13

barbiellinii (Ceresa), 1934 (Quichuana), Habromyia

46.100

barbiellinii Curran, 1936, Microdon ......................

46.63

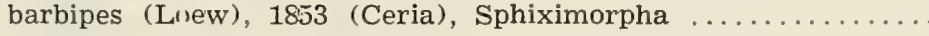

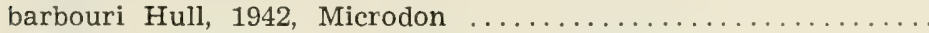

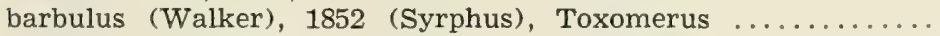

barrettoi (Carrera, Lopes \& Lane), 1947 (Schizoceratomyia),

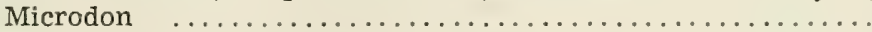

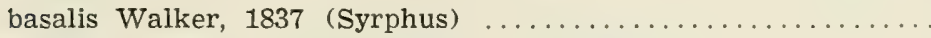

46.93

46.63

46.48

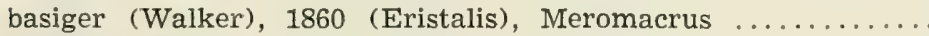

basilaris (Wiedemann), 1830 (Syrphus), Toxomerus ..........

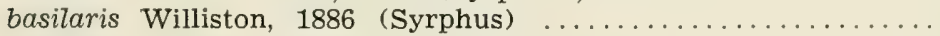

bassleri (Curran), 1939 (Volucella), Copestylum .............

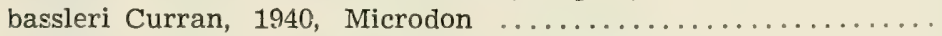

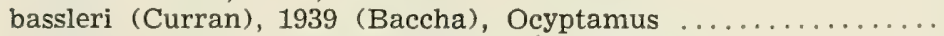

bassleri (Curran), 1941 (Cerioides), Polybiomyia ............

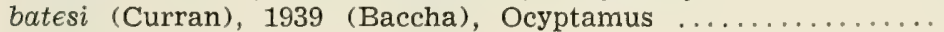

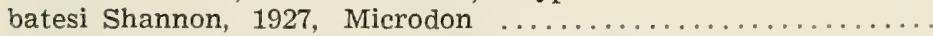

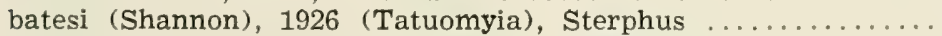

beatricea (Hull), 1950 (Volucella), Copestylum .............

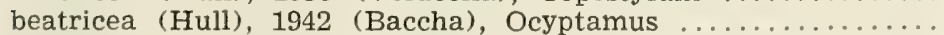

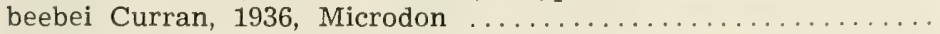

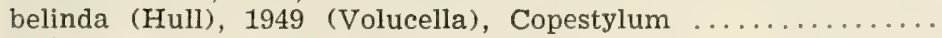

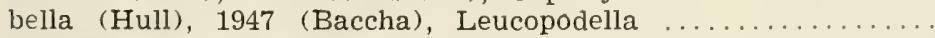

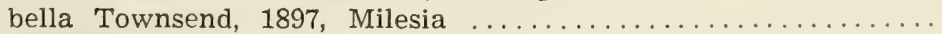

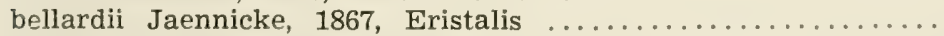

bellula (Williston), 1882 (Chrysogaster), Orthonevra ........

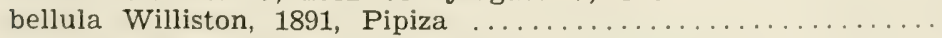

bellulum (Williston), 1891 (Phalacromyia), Copetylum .......

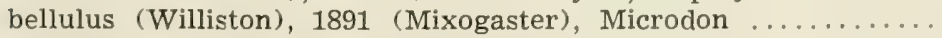

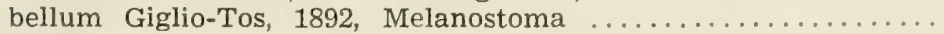

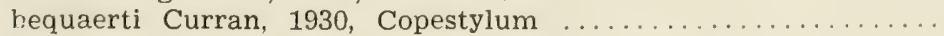

bequaerti (Curran), 1936 (Volucella), Copestylum ..........

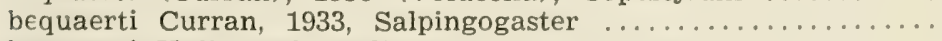

bequaerti Hull, 1956, Mallota

46.61

46. 39

46.111

46.48

46.39

46.72

46.63

46.13

46.95

46.22

46.63

46.117

46.72

46.13

46.63

46.72

46.46

46.120

46.101

46.91

46.67

46.72

46.63

46.42

46.72

46.73

46. 31

46.99

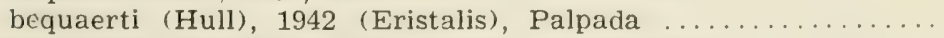

46.103

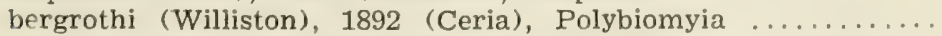

46.95

berthae Lima, 1946, Rhysops

46.42

46.66

bertrandi (Austen), 1913 (Melanostoma), Carposcalis

46.40 


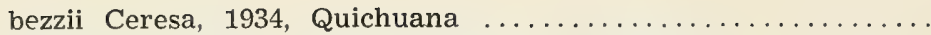

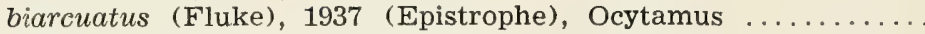

46.98

bicincta (Bigot), 1884 (Mesograpta?), Allograpta ............

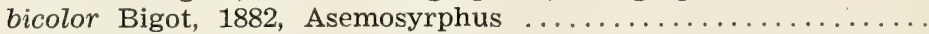

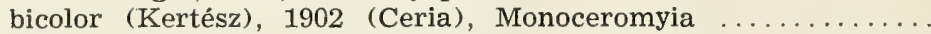

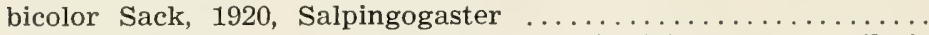

bicolor (Shannon \& Aubertin), 1933 (Chilosia), Chromocheilosia

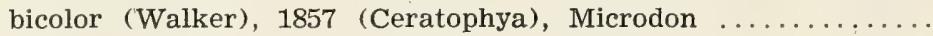

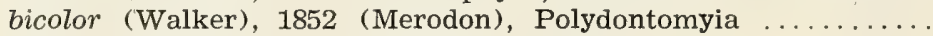

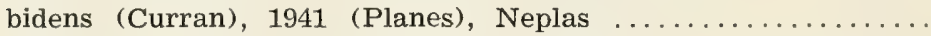

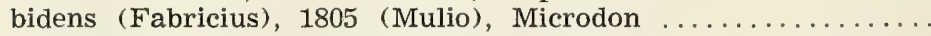

bidentatus (Giglio-Tos), 1893 (Mesogramma), Toxomerus .....

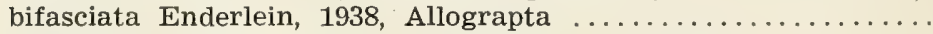

bifasciata (Macquart), 1855 (Eristalis), Palpada ............

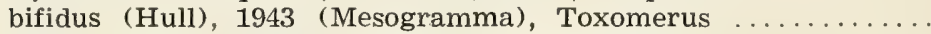

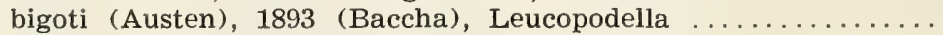

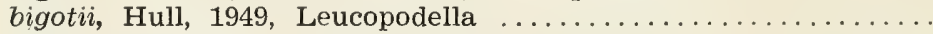

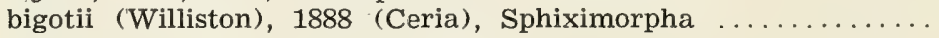

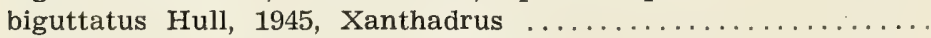

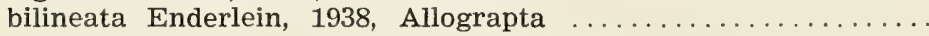

bilineella Enderlein, 1938, Allograpta ................

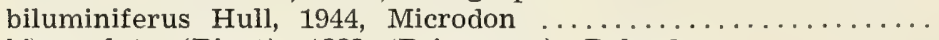

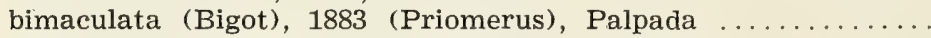

bimaculata Enderlein, 1938, Allograpta ..................

bimaculatum (Sack), 1941 (Phalacromyia), Copestylum ......

binominatum (Goot), 1964 (Volucella), Copestylum ..........

bipunctatum (Hull), 1941 (Phalacromyia), Copestylum .......

bipunctatus (Hull), 1943 (Mesogramma), Toxomerus .........

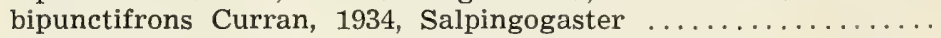

bipunctipennis (Hull), 1942 (Baccha), Leucopodella ..........

bisinterrupta (Enderlein), 1938 (Fazia), Allograpta .........

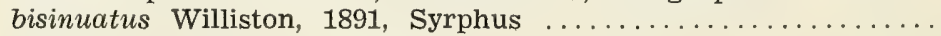

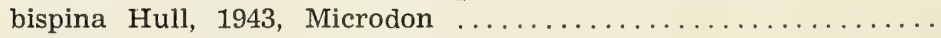

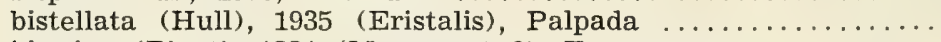

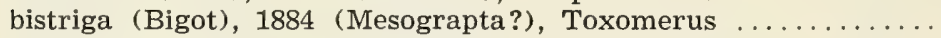

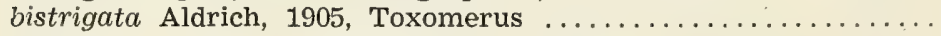

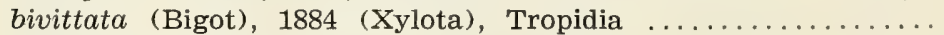

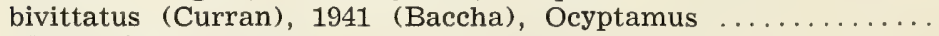

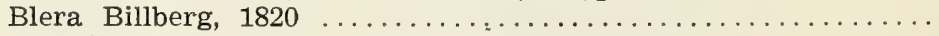

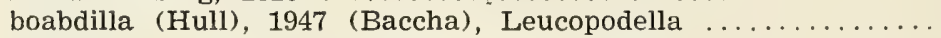

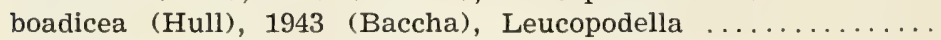

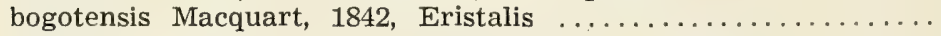

bolivariensis (Fluke), 1945 (Melanostoma), Rhysops .........

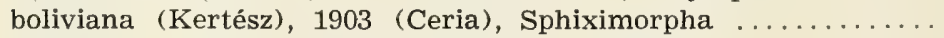

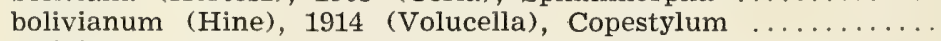

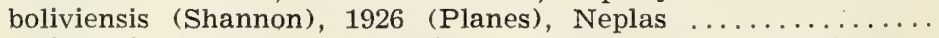

boliviensis (Shannon), 1927 (Halictomyia), Trichopsomyia ...

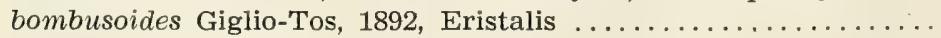

bonariensis (Brèthes), 1905 (Baccha), Ocyptamus ..........

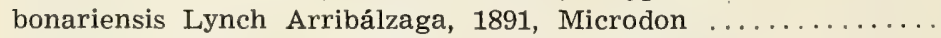

bonariensis Lynch Arribálzaga, 1892, Nausigaster ........... boraceiensis (Papavero), 1962 (Ceratophya), Argentinomyia ... borgmeieri Lane \& Carrera, 1944, Quichuana ...............

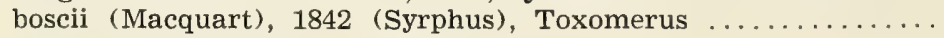

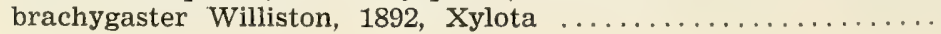

46.14

46.34

46.96

46.94

46.31

46.90

46.63

46.96

46.119

46.63

46.48

46.37

46.105

46.48

46.46

46.46

46.93

46.44

46.34

46.34

46.64

46.109

46.36

46.72

46.72

46.72

46.48

46.31

46.46

46.34

46.8

46.64

46.103

46.48

46.48

46.120

46.13

46.119

46.46

46.46

46.101

46.42

46.93

46.72

46.114

46.68

46.102

46.13

46.64

46.92

46.57

46.98

46.49

46.114 
Brachyopa Meigen, 1822 ...................... 46.92

bradleyi (Curran), 1925 (Volucella), Copestylum .......... 46.72

brauerii (Williston), 1888 (Ceria), Sphiximorpha .......... 46.93

Braziliana Curran, 1925 ......................... 46.42

braziliana (Shannon), 1927 (Eumyiolepta), Myolepta ....... 46.90

brazilianum (Hull), 1938 (Volucella), Copestylum .......... 46.72

braziliensis (Curran), 1939 (Baccha), Ocyptamus ........... 46.13

braziliensis (Goot), 1964 (Eristalis), Palpada .............. 46.103

brethesi (Shannon), 1928 (Lepidostola), Lepidomyia ......... 46.88

breve (Giglio-Tos), 1892 (Volucella), Copestylum .......... 46.72

brevicera Hull, 1946, Quichuana ...................... 46.99

brevifacia (Hull), 1943 (Mesogramma), Toxomerus ........ 46.49

brevifacies (Curran), 1934 (Volucella), Copestylum ........ 46.72

brevifacies (Hull), 1943i (Mesogramma), Toxomerus ......... 46.49

brevipennis (Schiner), 1868 (Baccha), Ocyptamus .......... 46.13

brevivillosa (Hull), 1937 (Eristalis), Palpada .............. 46.103

brevivittatum (Curran), 1930 (Volucella), Copestylum ........ 46.72

bromleyi (Curran), 1929 (Baccha), Ocyptamus ........... 46.14

browni Curran, 1941, Salpingogaster .............. 46.31

browni Fluke, 1942, Allograpta ...................... 46.34

browni (Fluke), 1945 (Melanostoma), Rhysops ........... 46.42

browni Fluke, 1943, Tuberculanostoma .................. 46.45

bruchi Shannon, 1927, Microdon .................... 46.64

bruesi Hull, 1945, Microdon ...................... 46.64

bruneri (Curran), 1939 (Volucella), Copestylum .......... 46.72

bruneri Curran, 1936, Meromacrus ................ 46.111

bruneri Curran, 1932, Salpingogaster ................ 46.31

brunneola Frey, 1946 (Neoscaeva), Allograpta ............. 46.35

brunneus Hull, 1942, Meromacrus ...................... 46.111

brunnicolor (Hull), 1938 (Volucella), Copestylum .......... 46.72

brunnigaster (Hull), 1943 (Volucella), Copestylum ........... 46.72

brunnipennis Hull, 1944, Microdon ................. 46.63

brunnipennis (Hull), 1942 (Baccha), Ocyptamus ........... 46.14

brutus Hull, 1944, Microdon ....................... 46.64

bucephalus (Wieđemann), 1830 (Syrphus), Xanthandrus ...... 46.44

bulbosum (Fluke), 1951 (Volucella), Copestylum .......... 46.73

bullaephora (Shannon), 1927 (Fazia), Allograpta .......... 46.34

cacica (Walker), 1860 (Ceria), Monoceromyia ............. 46.94

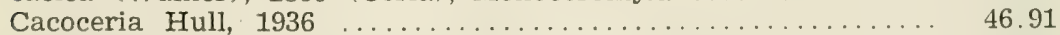

Cacomyia Hull, $1930 \ldots \ldots \ldots \ldots \ldots \ldots \ldots \ldots \ldots \ldots . \ldots \ldots . \ldots \ldots$

caesar Curran, 1940, Microdon .................. 46.64

caesariatum (Wrilliston), 1891 (Volucella), Copestylum ...... 46.73

calathea Shannon, 1925, Quichuana ................. 46.98

calceolatus (Macquart), 1842 (Syrphus), Toxomerus .......... 46.49

caldus (Walker), 1852 (Syrphus), Ocyptamus .............. 46.14

caliginosa (Walker), 1849 (Eristalis), Palpada ............. 46.103

calla (Curran), 1941 (Baccha), Ocyptamus .............. 46.14

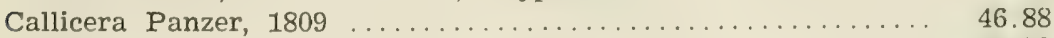

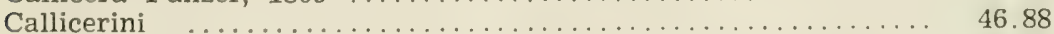

callidus (Hine), 1914 (Baccha), Ocyptamus .............. 46.14

calliphoroides (Bigot), 1885 (Dimorphomyia), Asemosyrphus ... 46.97

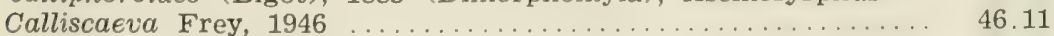

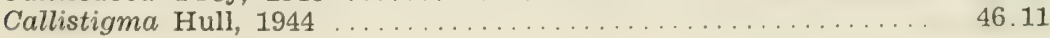




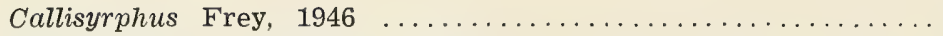

46.11

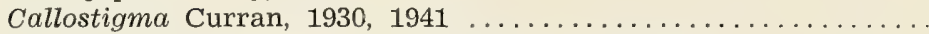

46.11

calochaetum (Hull), 1941 (Volucella), Copestylum ......... 46.73

calomera (Bigot), 1880 (Eristalomyia), Palpada ........... 46.104

calops (Bigot), 1880 (Eristalomyia), Palpada ............. 46.104

calopus Loew, 1864, Lepidomyia .................. 46.88

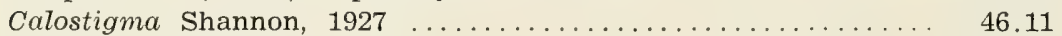

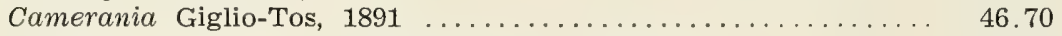

camilla (Hull), 1951 (Mesogramma), Toxomerus .......... 46.49

camposi (Curran), 1939 (Volucella), Copestylum .......... 46.73

camrasi (Sedman), 1965 (Valdivia), Valdiviomyia .......... 46.118

canusium (Walker), 1849 (Milesia), Meromacrus ........... 46.111

capitatus (Loew), 1863 (Baccha), Ocyptamus ............. 46.14

capitis (Curran), 1925 (Cerioides), Polybiomyia ........... 46.95

carinifacies Curran, 1934, Microdon ................ 46.59

carlota (Curran), 1929 (Baccha), Ocyptamus ............ 46.14

carmelita (Hull), 1948 (Baccha), Leucopodella ........... 46.46

carolae (Capelle), 1956 (Rhopalosyrphus), Microdon ........ 46.60

cüpenteri (Hull), 1945 (Ceratophya), Argentinomyia ....... 46.57

Carposcalis Enderlein, 1938 ......................... 46.40

carrerai (Papavero), 1962 (Masarygus), Microdon ......... 46.61

Carreramyia Doesburg, 1966 ...................... 46.61

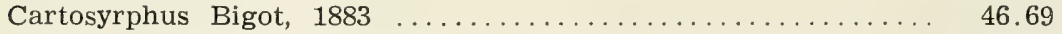

castanea (Bigot), 1875 (Volucella), Copestylum .......... 46.82

catabomba (Williston), 1891 (Melanostoma), Rhysops ....... 46.42

catharinensis (Enderlein), 1938 (Pipiza), Trichopsomyia ..... 46.68

cecrops (Hull), 1958 (Baccha), Ocyptamus ............... 46.14

celia (Hull), 1949 (Baccha), Ocyptamus ................. 46.14

centaureus (Hull), 1951 (Mesogramma), Toxomerus ........ 46.49

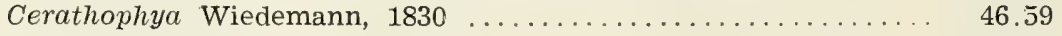

Ceratophya Wiedemann, $1824 \ldots \ldots \ldots \ldots \ldots \ldots \ldots \ldots \ldots \ldots \ldots . \ldots \ldots . \ldots \ldots$

cereberus (Hull), 1891 (Baccha), Ocyptamus ............. 46.14

ceres Hull, 1942, Meromacrus ......................... 46.111

Ceriodes, auctorum .............................. 46.93

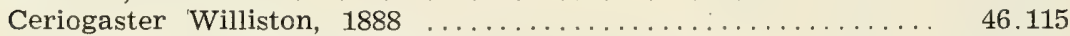

cerioides Hull, 1943, Microdon ..................... 46.64

Cerioides Rondani, $1850 \ldots \ldots \ldots \ldots \ldots \ldots \ldots \ldots \ldots \ldots \ldots . \ldots \ldots . \ldots \ldots . \ldots \ldots$

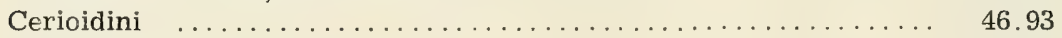

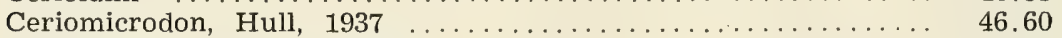

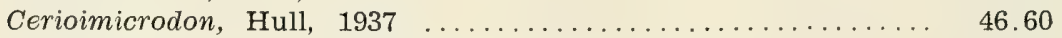

cestus Hull, 1946, Quichuana ....................... 46.98

chaetogaster (Hull), 1943 (Volucella), Copestylum .......... 46.73

chaetophorum (Williston), 1887 (Volucella), Copestylum ..... 46.73

chaetopoda (Davidson), 1922 (Melanostoma), Carposcalis :... 46.40

chalconota Philippi, 1865 (Syrphus), Carposcalis ........... 46.40

chalybescens (Wiedemann), 1830 (Volucella), Copestylum .... 46.73

Chamaesphegina Shannon \& Aubertin, 1933 .............. 46.91

Chamaesyrphus Mik, 1895 .......................... 46.69

championi (Williston), 1892 (Mallota), Quichuana ......... 46.98

chapadense (Curran), 1930 (Volucella), Copestylum ......... 46.73

chapadensis (Curran), 1930 (Baccha), Ocyptamus .......... 46.14

Chasmia Enderlein, 1938 ...................... 46.33

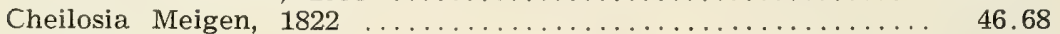

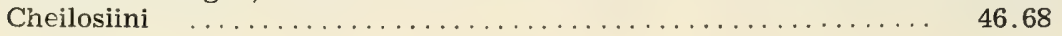


chilena (Rondani), 1863 (Eritalomya), Palpada ............

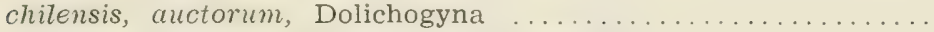

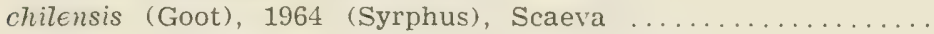

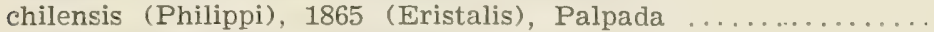

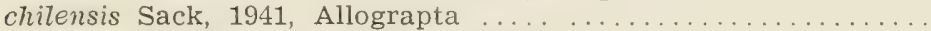
chilensis (Walker), 1837, Dolichogyna

46. 104

46.97

46.9

$46.10 t$

46.37

46.97

46.97

46.68

46.59

45.39

46. 122

46.114

46.117

46.73

46.90

46.64

46.92

46. 64

46.59

46.68

46.91

46.88

46.114

46.61

46.100

46.40

46.40

46.59

46.58

46.49

46.45

46.98

46. 73

46.111

46.112

46.73

46.102

46.90

46.52

46.73

46.112

46. 49

46.73

46. 49

46.11

46.33

46.73

46.61

46.104

46.67

46.101

46.73

46.49

$46.10 \mathrm{i}$

46. 10) 
clavicornis Curran, 1940, Microdon ................. 46.64

coarctatus Loew, 1864, Microdon ................... 46.64

coarctatus (Wiedemann), 1830 (Xylota), Sterphus ........ 46.117

cobboldia (Hull), 1958 (Baccha), Ocyptamus ............. 46.14

cochenillivorus (Guérin-Méneville), 1848 (Baccha), Ocyptamus . 46.14

cocheri Fluke, 1956, Toxomerus ................. 46.49

cockerelli (Curran), 1927 (Volucella), Copestylum ......... 46.73

coeruleus (Williston), 1891 (Baccha), Ocyptamus ......... 46.14

coeruleithorax Williston, 1888, Habromyia .............. 46.100

coerulescens Macquart, 1834, Senogaster ................ 46.119

coeruleus (Rondani), 1863 (Xylota), Sterphus ............ 46.116

coesariata (Giglio-Tos), 1892 (Volucella), Copestylum ....... 46.73

?cognata (Rondani), 1848 (Eristalis), Palpada ............ 46.39

colludens Walker, 1860 (Syrphus) . . . . . . . . . . . . . . . 46.39

colombia Curran, 1925, Allograpta ................... 46.34

colombia (Curran), 1929 (Lepidostola), Lepidomyia .......... 46.88

columbianum Enderlein, 1938 (Melanostoma) ............ 46.47

colombianus (Curran), 1941 (Baccha), Ocyptamus .......... 46.14

columbianus (Enderlein), 1938 (Braziliana), Rhysops ........ 46.42

columbianus Rondani, 1863 (Syrphus) ................. 46.39

colombiense Thompson, Copestylum ................... 46.73

colombii Macquart, 1850, Mallota ................... 46.100

columbica (Macquart), 1855 (Eristalis), Palpada ........... 46.104

columella Fluke, 1945, Rhysops .................... 46.43

comastes (Williston), 1891 (Volucella), Copestylum .......... 46.86

comma (Giglio-Tos), 1892. (Mesogramma), Toxomerus ........ 46.49

compactum (Curran), 1925 (Lepidopsis), Copestylum ........ 46.73

compressa Curran, 1941, Salpingogaster .............. 46.32

comstocki (Williston), 1887 (Volucella), Copestylum ....... 46.74

comstocki (Williston), 1882, Senogaster ................. 46.120

communis Walker, 1849, Xylota .................... 46.114

concinnum (Philippi), 1865 (Volucella), Copestylum ......... 46.74

concinnus (Williston), 1891 (Baccha), Ocyptamus .......... 46.15

concolor (Philippi), 1865 (Phalacromya), Copestylum ....... 46.80

concolor (Philippi), 1865 (Eristalis), Palpada .............. 46.104

conformis Loew, 1866, Ocyptamus .................... 46.15

confusus (Goot), 1964 (Baccha), Ocyptamus ............. 46.15

confusus (Schiner), 1868 (Mesogramma), Toxomerus ....... 46.49

congrua (Wulp), 1888 (Eristalis), Palpada .............. 46.104

conica (Fabricius), 1805 (Milesia), Palpada ............... 46.104

conifacium (Hull), 1943 (Volucella), Copestylum ........... 46.74

conjunctus (Wiedemann), 1830 (Syrphus), Ocyptamus ........ 46.15

connexus (Hull), 1949 (Baccha), Ocyptamus .............. 46.21

Conocotamyia Fluke, 1951 ..................... 46.97

conopida (Philippi), 1865 (Baccha), Salpingogaster ......... 46.32

conopoides Kertész, 1910, Mixogaster ................... 46.58

conops Curran, 1940, Microdon ..................... 46.64

conopseus, Williston, 1886, Mixogaster .............. 46.58

conopsoides Macquart, 1842, Mixogaster ............... 46.58

contigua Macquart, 1847, Sphaerophoria ............. 46.38

contumax (Curran), 1939 (Volucella), Copestylum ........... 46.74

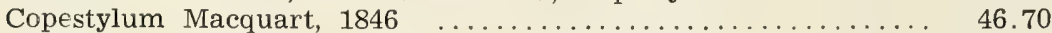

coquilletti (Williston), 1891 (Didea), Dideomima ........... 46.10

cora (Curran), 1941 (Baccha), Ocyptamus ............... 46.15

cora (Hull), 1949 (Eristalis), Palpada ................. 46.104 
corbiculipes (Papavero), 1962 (Hypselosyrphus), Microdon .... 46.61 cordelia (Hull), 1949 (Baccha), Ocyptamus .............. 46.15 cordiae (Townsend), 1897 (Volucella), Copestylum .......... 46.74 coreopsis (Hull), 1944 (Callistigma), Ocyptamus ........... 46.15 cornuta Hull, 1944, Salpingogaster ................. 46.31 corcna Curran, 1949, Microdon ................... 46.64 correctum (Curran), 1927 (Volucella), Copestylum ......... 46.74 cortesi (Marnef), 1967 (Austrosyritta), Syritta ............ 46.119 corumbense (Curran), 1930 (Volucella), Copestylum ........ 46.74 cosmia (Schiner), 1868 (Eristalis), Palpada ................ 46.104 costalis (Walker), 1837 (Pipiza), Ocyptamus .............. 46.19 costalis (Walker), 1852 (Amathia), Salpingogaster ......... 46.31 costalis (Wiedemann), 1830 (Syrphus), Toxomerus.......... 46.49 costatus (Say); 1829 (Baccha), Ocyptamus ................ 46.15 cothurnata Bigot, 1883, Salpingogaster ................46.31 crassicalx Enderlein, 1938 (Pachysphyria) .............. 46.47 crassipes (Fabricius), 1805 (Merodon), Meromacrus ......... 46.112 crassitarsis (Macquart), 1848 (Aphritis), Microdon ............ 46.64 crassus (Walker), 1852 (Baccha), Ocyptamus .............. 46.15 craverii (Giglio-Tos), 1892 (Volucella), Copestylum ........... 46.74 crenulatus (Williston), 1891 (Melanostoma), Rhysops ....... 46.42 Crepidomyia Shannon, 1926 ....................... 46.116 crepuscularium (Hull), 1942 (Volucella), Copestylum ......... 46.74 crepuscustellata (Hull), 1946 (Lepidostola), Lepidomyia ...... 46.88 cressoni (Hull), 1930 (Cacomyia), Cacoceria ............... 46.91

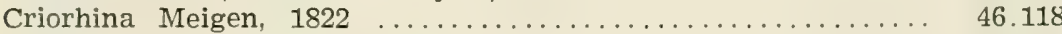

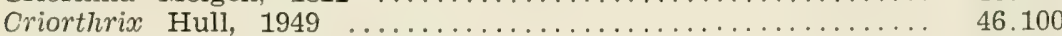
crocatus (Austen), 1893 (Baccha), Ocyptamus .............. 46.15 croceimaculata (Jacobs), 1900 (Eristalis), Palpada .......... 46.104 croceipes (Bigot), 1880 (Eristalomyia), Palpada ............ 46.104 croceus (Austen), 1893 (Baccha), Ocyptamus ............. 46.15 crockeri (Curran), 1934 (Mesogramma), Toxomerus ......... 46.49 croesus (Hull), 1940 (Mesogramma), Toxomerus ............ 46.49 cruciatum Bigot, 1884, Melanostoma .................. 46.42 cruciatum (Hull), 1943 (Volucella), Copestylum .......... 46.74 crucifer Bigot, 1847, Meromacrus ..................... 46.111 cruciger (Wiedemann), 1830 (Milesia), Meromacrus ........ 46.111 crypticus (Hull), 1942 (Baccha), Ocyptamus ............... 46.15 Cryptineura Bigot, 1859 ........................ 46.91 cubana Curran, 1932, Allograpta ..................... 46.34 cubanus Fluke, 1936, Xanthandrus .................. 46.44 cubanus (Hull), 1943 (Baccha), Ocyptamus .............. 46.15 cubensis Curran, 1932, Mixogaster ................... 46.58 cubensis (Macquart), 1850 (Baccha), Ocyptamus .......... 46.15 cubensis (Macquart), 1842 (Eristalis), Palpada ............. 46.103 cubomaculatum (Hull), 1937 (Volucella), Copestylum ........ 46.74 cultratus (Austen), 1893 (Baccha), Ocyptamus ............ 46.15 cultrinus (Curran), 1939 (Baccha), Ocyptamus ............ 46.16 cuprescens (Hull), 1941 (Planes), Neplas ................ 46.114 cupricolor (Hull), 1948 (Volucella), Copestylum ............ 46.74 cuprinus (Bigot), 1884 (Mesograpta?), Toxomerus ........... 46.53 curiosum (Curran), 1939 (Volucella), Copestylum ........... 46.74 currani (Fluke), 1951 (Volucella) Copestylum .............4 46.74 currani Fluke, 1937, Rhysops .................... 46.43 currani Fluke, 1937, Trichopsomyia .................. 46.68 currani Goot, 1964, Microdon ...................... 46.64 


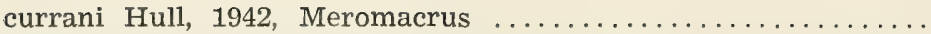

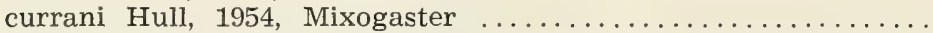

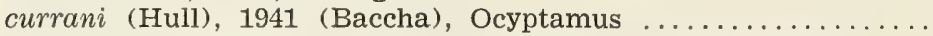
curvipes (Wiedemann), 1830 (Merodon), Polydontomyia ......

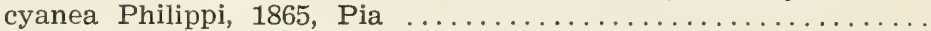

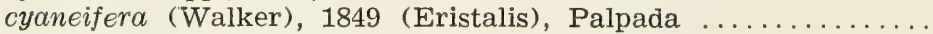
cyaneiventris (Macquart), 1846 (Aphritis), Microdon .........

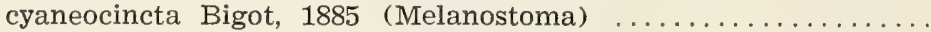

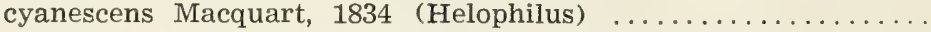
cyanescens (Macquart), 1842 (Volucella), Copestylum ........

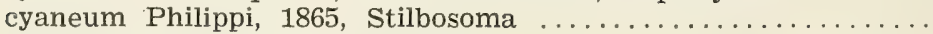

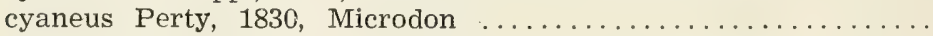
cyanocephala (Philippi), 1865 (Sterphus), Philippimyia ....... cyanoproctum (Curran), 1939 (Volucella), Copestylum ....... cyanoprora (Curran), 1939 (Volucella), Copestylum ..........

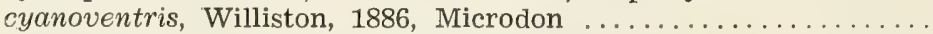

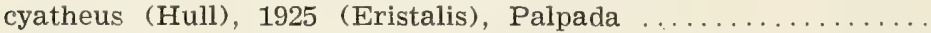

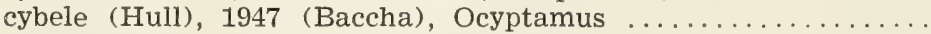

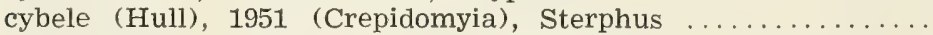

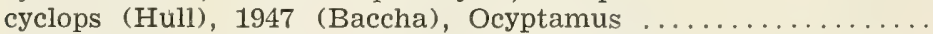

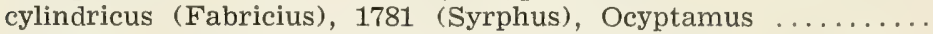

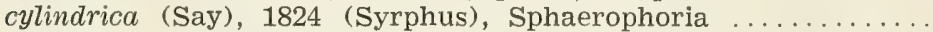

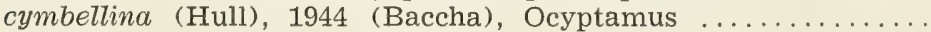

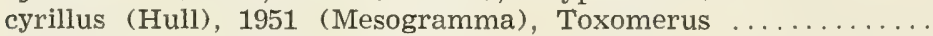

dactylopiana Blanchard, 1938, Salpingogaster ............

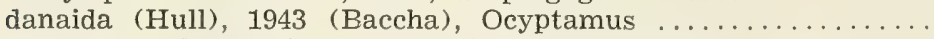
daphnaeus (Walker), 1849 (Ceria), Monoceromyia .......... darlingtoni (Hull), 1944 (Crepidomyia), Sterphus ............ darwini (Shannon), 1927 (Valdivia), Valdiviomyia ..........

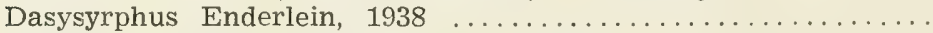

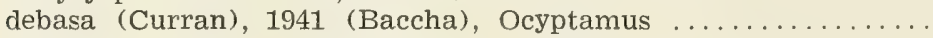
deceptor (Curran), 1925 (Phalacromyia), Copestylum ........

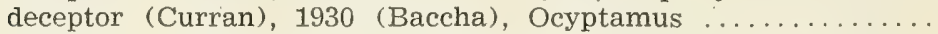

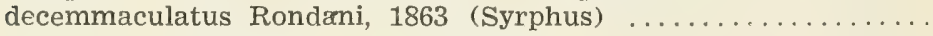

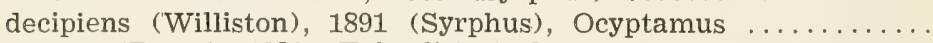

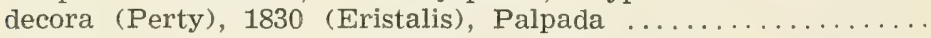

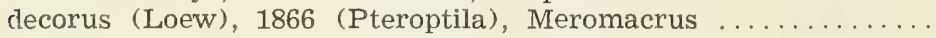
dejongi Doesburg, 1958, Allograpta .................. delicatissimus (Hull), 1943 (Baccha), Ocyptamus ........... delicatula (Hull), 1943 (Baccha), Leucopodella .............

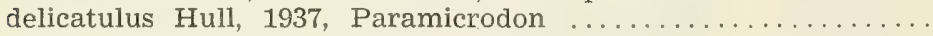

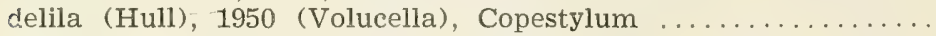

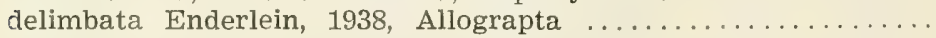

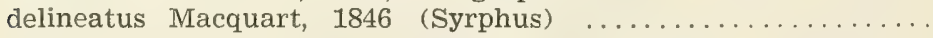

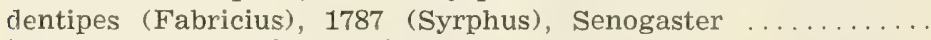

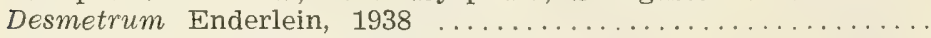

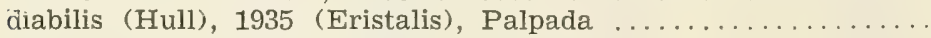

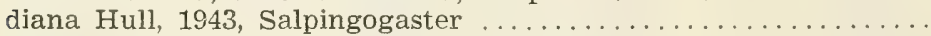

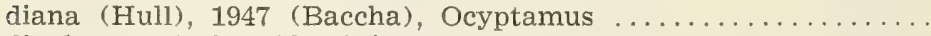

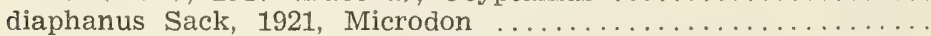

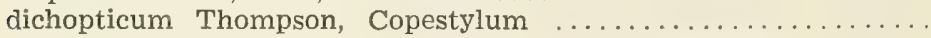
dichroicum (Giglio-Tos), 1892 (Volucella), Copestylum ........

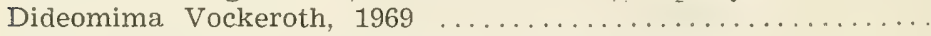
difficilis (Curran), 1930 (Mesogramma) Toxomerus .......... diffusus (Curran), 1939 (Baccha), Ocyptamus

46.33

46.16

46.94

46.117

46.118

46.10

46.16

46.74

46.16

46.39

46.16

46.104

46.112

46.37

46.16

46.46

46.57

46.74

46.34

46.39

46.119

46.91

46.104

46.31

46.16

46.64

46.74

46.74

46.10

46.49

46.16 
dimidiata Giglio-Tos, 1892, Mixogaster .............. 46.58

dimidiatus (Fabricius), 1781 (Syrphus), Ocyptamus ........ 46.16

diminuta (Walker), 1849 (Eristalis), Palpada ............. 46.104

demitiata, Fluke, 1957, Mixogaster ...................... 46.58

dimorphium (Curran), 1939 (Volucella), Copestylum ........ 46.74

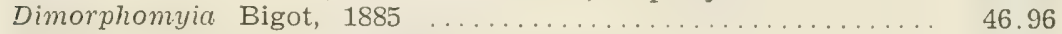

dion (Hull), 1951 (Crepidomyia), Sterphus .............. 46.117

dionysianna (d'Andretta \& Carrera), 1952 (Lepidostola), Lepidomyia

Dioprosopa Hull, 1949

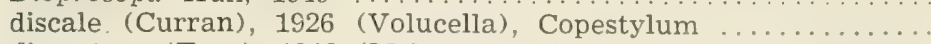

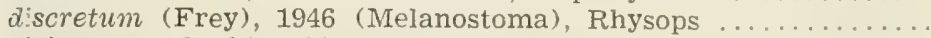

disjunctus (Sack), 1921 (Doros), Ocyptamus

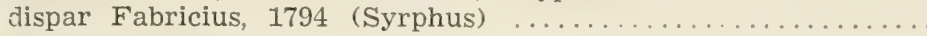

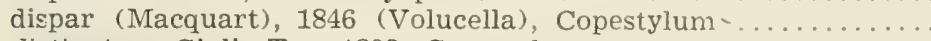

distinctum Giglio-Tos, 1892, Copestylum

distinguenda (Wiedemann), 1830 (Eristalis), Palpada ........

diversifasciatus (Knab), 1914 (Syrphus), Ocyptamus .............

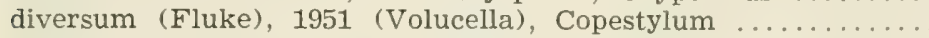

diversus (Gigiio-Tos), 1892 (Mesogramma), Toxomerus ........

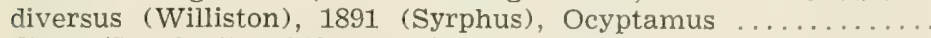

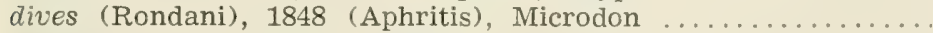

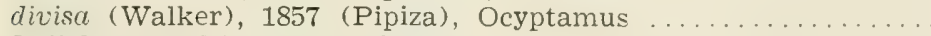

Dolichogyna Macquart, 1842

Doliosyrphus Bigot, 1882

46.88

46.10

46.75

46.43

46.17

46. 39

46.75

46.78

46.104

46.17

46.71

46.50

46.17

46.65

46.16

46.97

46.102

dolorosa (Hull), 1941 (Sarolepta), Myolepta ............. 46.89

dolorosa Hull, 1946, Quichuana ..................... 46.98

dolorosus (Hull), 1950 (Baccha), Ocyptamus ............ 46.17

dolosa (Walker), 1857 (Pipiza), Ocyptamus ............. 46.16

dolosa (Williston), 1891 (Baccha), Ocyptamus ............ 46.30

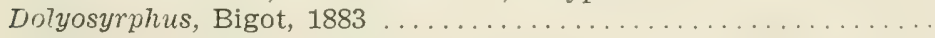

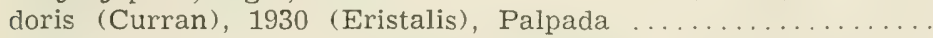

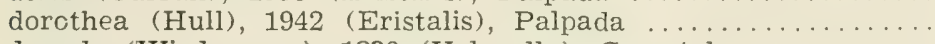

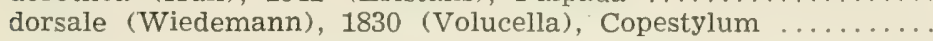

dracaena (Curran), 1947 (Volucella), Copestylum ...........

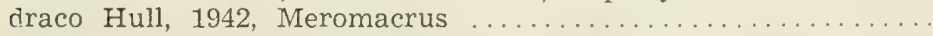

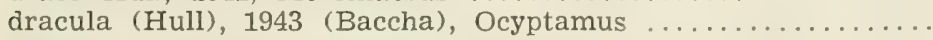

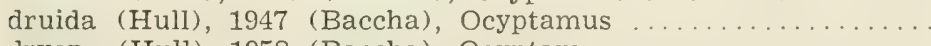

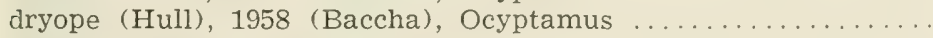

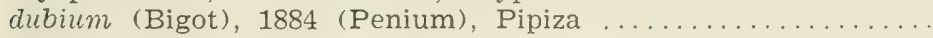

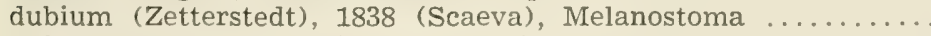

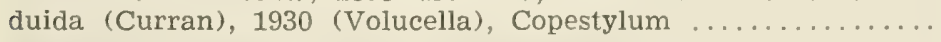

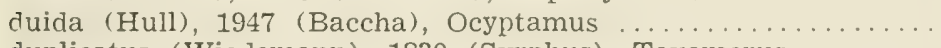

duplicatus (Wiedemann), 1830 (Syrphus), Toxomerus ........

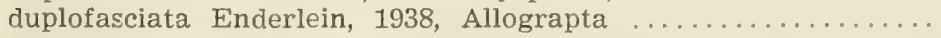

46.102

46.105

46.105

46.75

46.75

46.112

46.17

46.17

46.17

46.68

46.42

46.75

46.17

46.50

46.34

eblis (Hull), 1943 (Styxia), Ocyptamus ............... 46.17

cetypus (Say), 1829 (Syrphus), Toxomerus ............. 46.50

ecuadorea (Fluke), 1951 (Volucella), Copestylum .......... 46.72

scuadoreus (Hull), 1943 (Mesogramma), Toxomerus ........ 46.50

ecuadoriensis (Fluke), 1945 (Melanostoma), Carposcalis ...... 46.41

edwardsi (Shannon \& Aubertin), 1933 (Melanostoma), Carposcalis 46.41

edwardsi (Shannon \& Aubertin), 1933 (Valdivia), Valdiviomyia 46.118

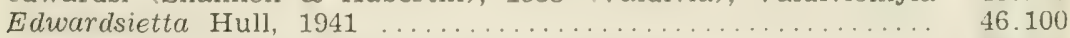

elegans (Blanchard), 1852 (Eritalis), Palpada ............ 46.105

elegans (Giglio-Tos), 1892 (Melanostoma), Ocyptamus ....... 46.17 


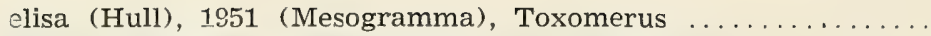

46.50

elnora (Shannon), 1927 (Calostigma), Ocyptamuś ...........

46.17

elongata (Hull), 1943 (Microdon), Argentinomyia ......... 46.57

elongatus (Hull), 1941 (Mesogramma), Toxomerus .......... 46.50

Elophilus Meigen, 1803 ....................... 46.101

emeralda (Hull), 1943 (Volucella), Copestylum ........... 46.75

emeralda Hull, 1943, Microdon ................... 46.64

emilia (Curran), 1939 (Volucella), Copestylum ............ 46.75

Eosalpingogaster Hull, 1949 ....................... 46.32

Eoseristalis Kanervo, 1938 ......................... 46.101

ephippium (Osten Sacken), 1875 (Mixtemyia), Spilomyia ...... 46.121

erebus (Hull), 1943 (Baccha), Ocyptamus .............. 46.17

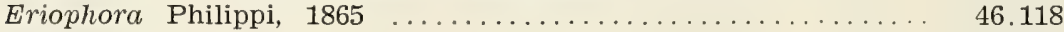

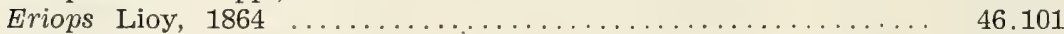

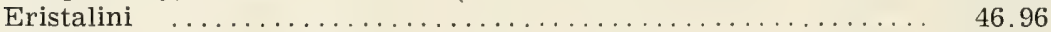

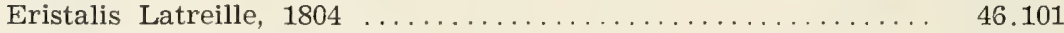

Eristaloides Rondani, 1845 ....................... 46.101

Eristalomya Rondani, 1857 ........................ 46.101

ernesta (Curran), 1926 (Volucella), Copestylum ............. 46.75

ernestina (Curran), 1930 (Volucella), Copestylum ........... 46.75

erratica (Curran), 1930 (Eristalis), Palpada .............. 46.105

erraticus (Williston), 1888 (Syrphus), Ocyptamus .......... 46.17

eruptova (Hull), 1943 (Baccha), Ocyptamus ............. 46.17

escomeli (Curran), 1929 (Volucella), Copestylum ........... 46.75

estebana (Townsend), 1895 (Volucella), Copestylum ........ 46.78

estrelita (Hull), 1948 (Baccha), Leucopodella ............. 46.46

esuriens (Fabricius), 1794 (Syrphus), Copestylum ........... 46.75

euceratus (Bigot), 1884 (Melanostoma), Xanthandrus ........ 46.45

eugenia (Williston), 1887 (Volucella), Copestylum ........... 46.75

euglossoides Gray, 1832, Microdon ................. 46.65

Eumerini .................................... 46.92

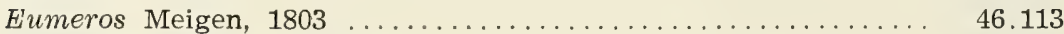

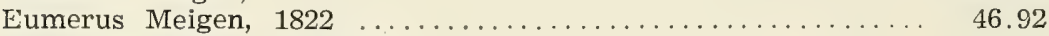

Eumicrodon Curran, $1925 \ldots \ldots \ldots \ldots \ldots \ldots \ldots \ldots \ldots \ldots . \ldots \ldots . \ldots \ldots$

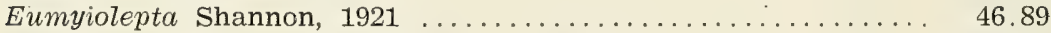

eupeltata (Bigot), 1884 (Syrphus), Allograpta ............ 46.34

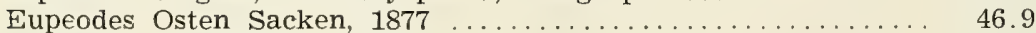

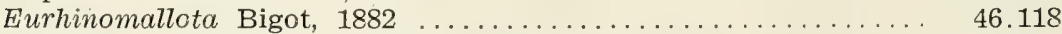

eurydice (Hull), 1951 (Mesogramma), Toxomerus ......... 46.50

Eurypterosyrphus Barretto \& Lane, $1947 \ldots \ldots \ldots \ldots \ldots \ldots$. . . . . . . . 7

eutristis Curran, 1925, Microdon ................... 46.64

evansi Thompson, 1968, Myolepta ................. 46.90

excavatus Rondani, 1850 (Syrphus) ................ 46.39

exeugenia (Curran), 1953 (Volucella), Copestylum ......... 46.75

exiguus (Williston), 1888 (Baccha), Ocyptamus ............ 46.18

exinanita (Gmelin), 1790 (Musca), Syrphus ............... 46.39

exotica (Wiedemann), 1830 (Syrphus), Allograpta .......... 46.34

expicta (Walker), 1860 (Eristalis), Palpada .............. 46.105

externum (Curran), 1939 (Volucella), Copestylum ........... 46.75

extrapolatus (Hull), 1943 (Mesogramma), Toxomerus ........ 46.50

facialis (Thomson), 1869 (Baccha), Pseudodoros .......... 46.11

facialis (Kertész), 1903 (Ceria), Sphiximorpha ........... 46.93

falcata Fluke, 1942, Allograpta ..................... 46.35

falcatus Williston, 1887 , Microdon ................ 46.64

familiaris (Walker), 1860 (Eristalis), Palpada ........... 46.105 
fasciata Curran, 1932, Allograpta ................. 46.35

fasciata Macquart, 1842, Dolichogyna ................ 46.97

fasciata (Sack), 1941 (Myiatropa), Quichuana ............ 46.98

fasciata (Wiedemann), 1819 (Eristalis), Palpada ............ 46.105

fasciatum (Macquart), 1842 (Volucella), Copestylum .......... 46.87

fasciatus (Hull), 1948 (Mesogramma), Toxomerus .......... 46.52

fasciatus Roeder, 1885, Ocyptamus ................ 46.18

fasciatus Walker, 1849, Helophilus ................... 46.96

fasciculata (Curran), 1938 (Eristalis), Palpada ................ 46.105

fascifrons (Macquart), 1846 (Syrphus), Allograpta .......... 46.35

foscithorax (Macquart), 1850 (Eristalis), Palpada .......... 46.109

fascithorax Williston, 1888, Ceriogaster .............. 46.115

fasciventris Rondani, 1850 (Syrphus) ................... . 46.39

fastigatus Fluke, 1945, Rhysops ....................... 46.43

fax (Townsend), 1895 (Volucella), Copestylum .......... 46.78

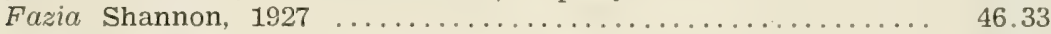

felix (Shannon), 1927 (Baccha), Ocyptamus ............. 46.22

femina (Curran), 1930 (Volucella), Copestylum .............. 46.85

femorata (Macquart), 1842 (Eristalis), Palpada ........... 46.106

femoratus Shannon, 1927, Alipumilio ................ 46.92

fenestrata (Macquart), 1842 (Syrphus), Carposcalis ........ 46.41

fenestratus Bigot, 1885, Ocyptamus ..................... 46.19

fenestratus Hull, 1943, Microdon ................... 46.64

fenestratus (Hull), 1949 (Baccha), Ocyptamus ........... 46.19

ferroxida (Hull), 1942 (Mesogramma), Toxomerus ......... 46.52

fervidus (Austen), 1893 (Baccha), Ocyptamus ............. 46.18

fervida (Fluke), 1945 (Melanostoma), Talahua ............ 46.45

festivus Fluke, 1945, Rhysops ..................... 46.43

fiametta (Hull), 1943 (Baccha), Ocyptamus ............ 46.18

filii (Doesburg), 1966 (Baccha), Ocyptamus ............. 46.18

filiolus (Shannon), 1927 (Baccha), Ocyptamus ............ 46.18

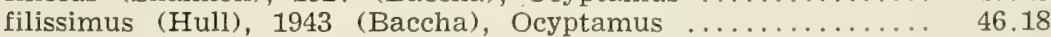

flammarius (Hull), 1943 (Mesogramma), Toxomerus ........ 46.48

flammineus (Hull), 1941 (Mesogramma), Toxomerus ........ 46.50

flatus (Hull), 1940 (Baccha), Ocyptamus ................. 46.18

flava Sack, 1941 (Ceratophya) ......................... 46.122

flavens (Austen), 1893 (Baccha), Ocyptamus ............. 46.18

flavibucca Enderlein, 1938, Allograpta ............... 46.35

flavicornis (Philippi), 1865 (Baccha), Ocyptamus .......... 45.25

flavifacies Shannon, 1927, Habromyia .................. 46.100

flavifrons Walker, 1849 , Xylota .......................... 46.114

flavigaster Hull, 1944 (Syrphus) . . . . . . . . . . . . . . . 46.39

flavimana Philippi, 1865, Tropidia ................ 46.120

flavipenne (Wiedernann), 1830 (Volucella), Copestylum ..... 46.75

flavipennis Curran, 1925, Microdon .................... 46.64

flavipennis (Macquart), 1842 (Eristalis), Palpada ............ 46.105

flavipennis (Wiedemann), 1830 (Syrphus), Ocyptamus ....... 46.18

flavipes (Carrera, Lopes \& Lane), 1947 (Schizoceratomyia), Microdon

flavipes (Enderlein), 1938 (Posthosyrphus), Metasyrphus ......

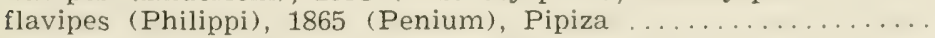

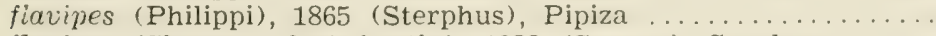

flavipes (Shannon \& Aubertin), 1933 (Scaeva), Syrphus ......

flavipleura Hull, 1943, Toxomerus

46.61

46.9

46.67

46.67

46.8

46.50

46.50

flavissimum (Giglio-Tos), 1892 (Volucella), Copestylum ...... 46.75 


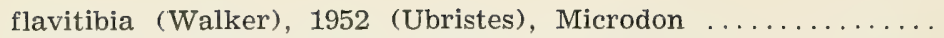

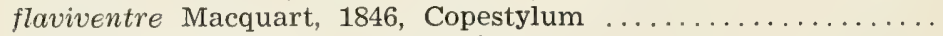

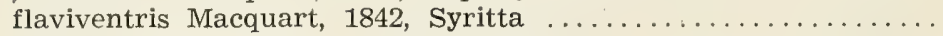

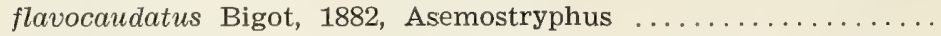
flavocuneus (Hull), 1940 (Mesogramma), Toxomerus .......

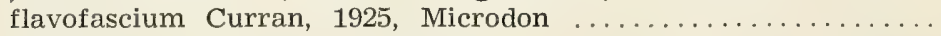
flavolinea Hull, 1949, Meromacrus flavoluna Hull, 1943, Microdon

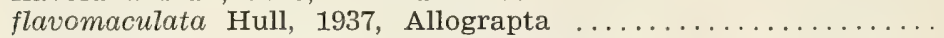

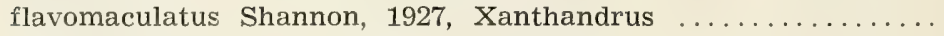

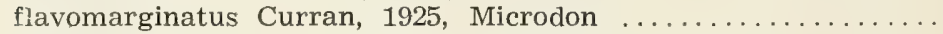
flavophylla (Hull), 1943 (Rhinoprosopa), Allograpta ........ flavopocula (Hull), 1948 (Mesogramma), Toxomerus .........

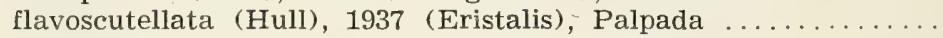
flavosignata (Kertész), 1902 (Ceria), Sphiximorpha ........

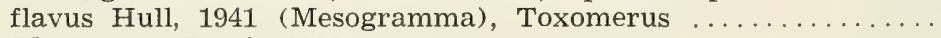
Flexineura Bezzi, 1908

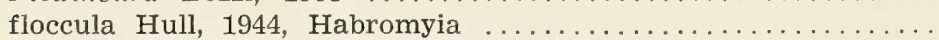

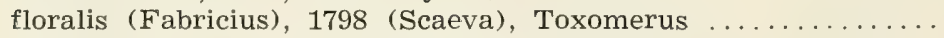

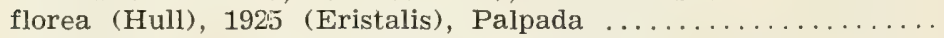

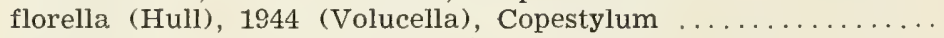

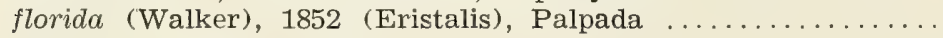

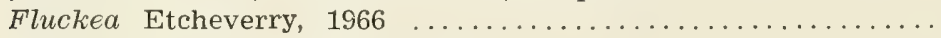

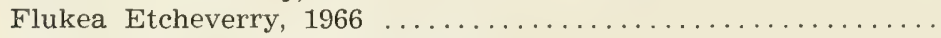

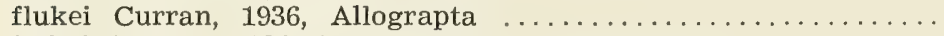

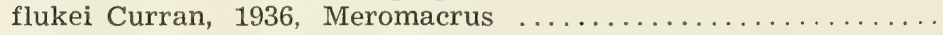

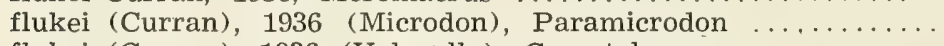

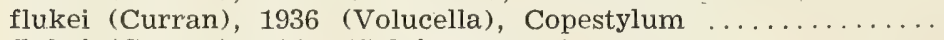
flukei (Curran), 1941 (Salpingogaster), Ocyptamus .......... flukei Curran, 1941, Nausigaster . . . . . . . . . . . . . . .

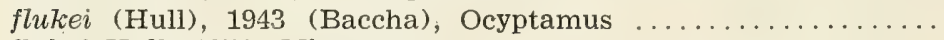

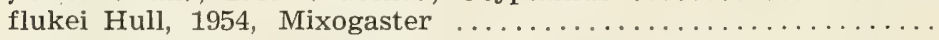
flukei (Sedman), 1964 (Chrysogaster), Orthonevra ..........

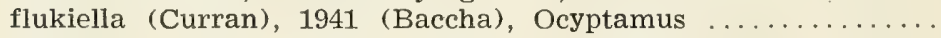
fornax (Townsend), 1895 (Volucella), Copestylum ............ formalis (Walker), 1849 (Helophilus), Asemosyrphus ......... forreri (Giglio-Tos), 1893 (Sphaerophoria), Allograpta .......

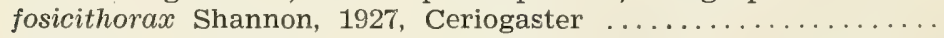

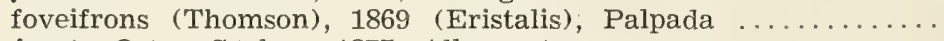

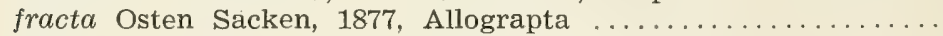
fractum (Curran), 1926 (Volucella), Copestylum ............

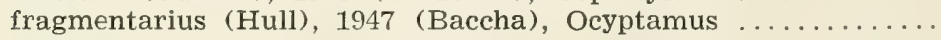
fraternus Bigot, 1883, Geyptamus . . . . . . . . . . . . .

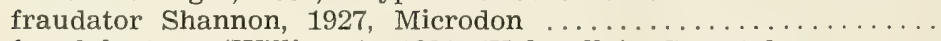
fraudulentum (Williston), 1891 (Volucella), Copestylum ....... frauenfeldi (Schiner), 1868 (Temnocera), Copestylum ........

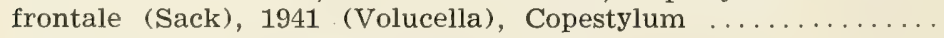

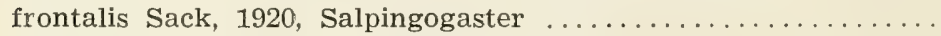

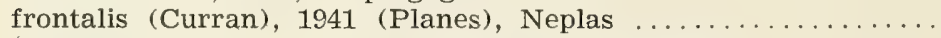
frontosa (Bigot), 1884 (Cartosyrphus), Cheilosia ............ fucatissimus (Hull), 1937 (Syrphipogon), Microdon .........

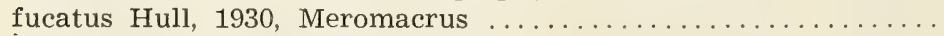

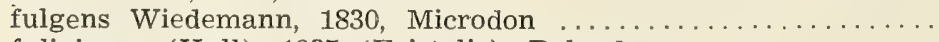

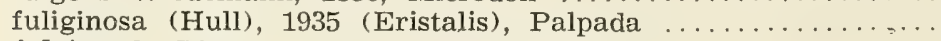
fulvicauda Bigot, 1884, Sphaerophoria

46.61

46.75

46.119

46.97

46.48

46.65

46.112

46.65

46.33

46.44

46.65

45.35

46.47

46.105

46.93

46.50

46.31

46.100

46.50

46.105

46.76

46.106

46.118

46.118

46.35

46.112

46.57

46.76

46.18

46.92

46.24

46.58

46.91

46.18

46.76

46.96

46.35

46.115

46.107

46.35

46.76

46.18

46.18

46.61

46.76

46.76

46.76

46.31

46.114

46.69

46.65

46.112

46.65

46.105

46. 38 
fulvicorne (Bigot), 1883 (Volucella), Copestylum ......... fulvicorne (Bigot), 1884 (Temnocera), Copestylum ..........

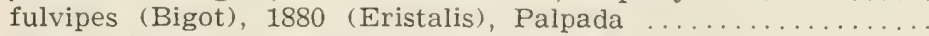
fulvitarsis (Rondani), 1863 (Eristalis), Palpada .............

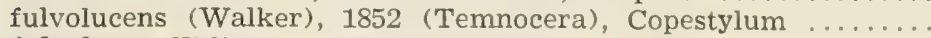
fulvolucus Williston, 1887, Copestylum

fulvonotatum (Bigot), 1875 (Volucella), Copestylum .........

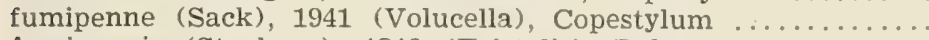

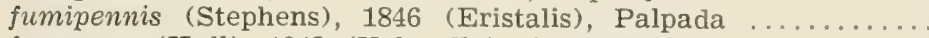

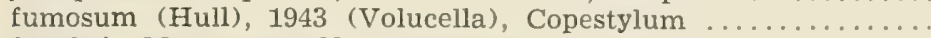
funebris Macquart, 1834, Ocyptamus

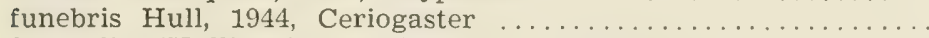

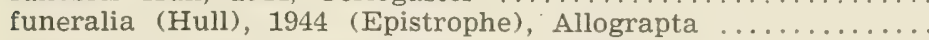
funerea (Rondani), 1850 (Eristalis), Palpada

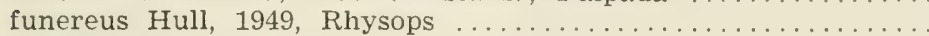
funereus Shannon \& Aubertin, 1933, Meromacrus ...........

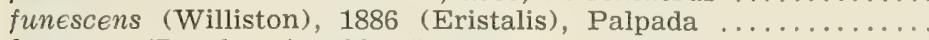
funestus (Doesburg), 1966 (Mesograpta), Toxomerus ........

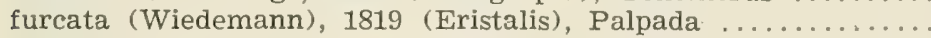

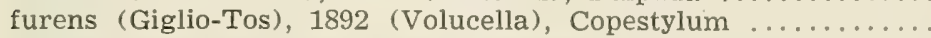

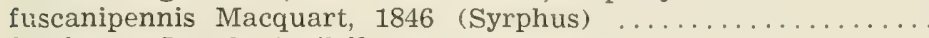
fuscicosta Lynch Arribálzaga, 1891, Ocyptamus ............ fuscipenne (Macquart), 1842 (Volucella), Copestylum ........ fusc.pennis (Macquart), 1850 (Volucella), Copestylum ........

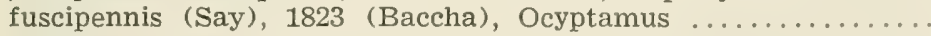

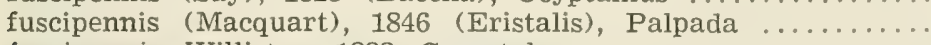

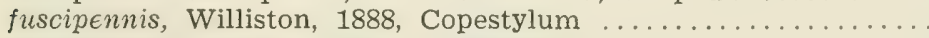

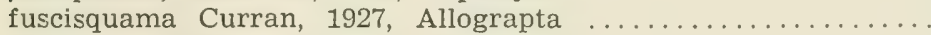

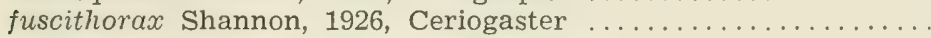

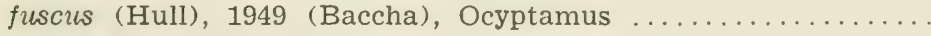

gagathina Bigot, 1887 (Priomerus) 


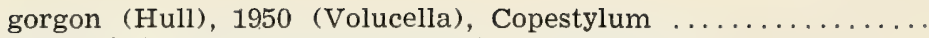
gowdeyi (Curran), 1926 (Baccha), Leucopodella ..............

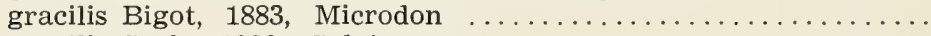

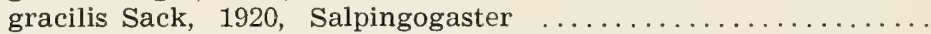
gracilis (Williston), 1891 (Baccha), Leucopodella ........... grandifemoralis (Curran), 1934 (Planes), Neplas ............... grandis Lynch Arribálzaga, 1892, Argentinomyia ............

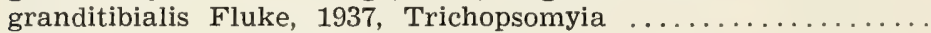
granulatum (Hull), 1944 (Volucella), Copestylum ...........

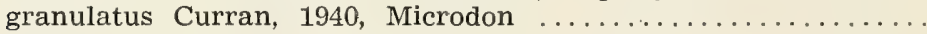

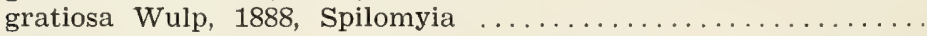

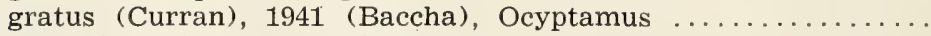

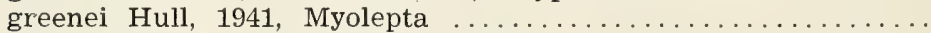

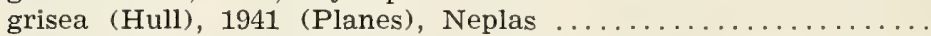

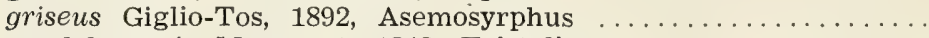
guadelupensis Macquart, 1842, Eristalis guentherii (Lynch Arribálzaga), 1891 (Holmbergia), Microdon guianicum (Hine), 1914 (Volucella), Copestylum .............

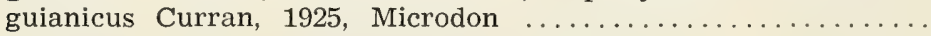

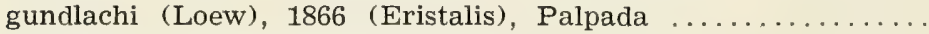
guttifer (Hull), 1943 (Mesogramma), Toxomerus ............

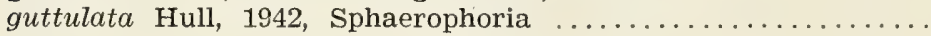

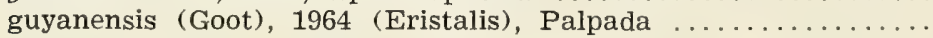

haagii (Jaennicke), 1867 (Volucella), Copestylum .........

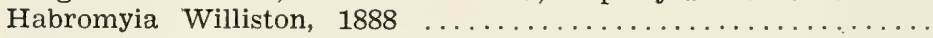
haemorrhoidalis (Philippi), 1865 (Priomerus), Myolepta .....

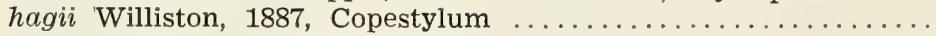
hahni (Bigot), 1888 (Helophilus), Dolichogyna .............

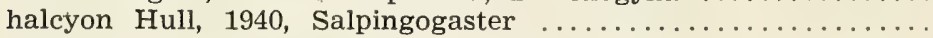

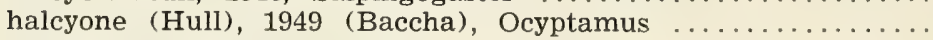

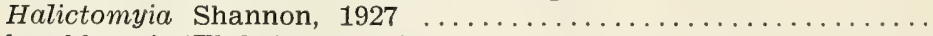
hambletoni (Fluke), 1951 (Volucella), Copestylum ..........

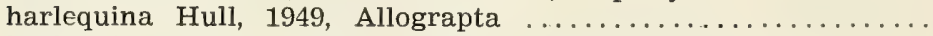

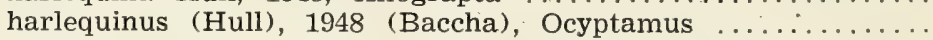

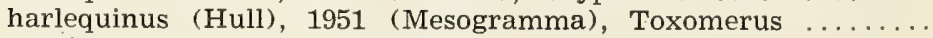

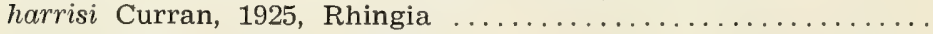

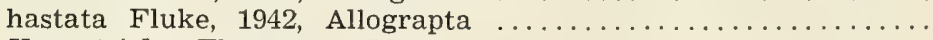

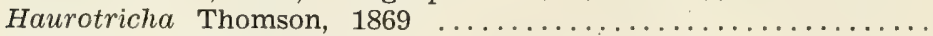

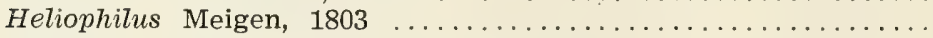

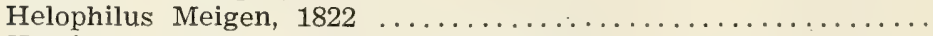
Hemixylota Shannon \& Aubertin, $1933 \ldots \ldots \ldots \ldots \ldots \ldots \ldots$

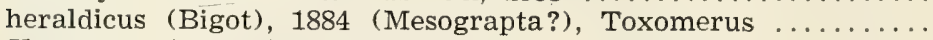

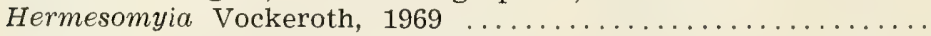

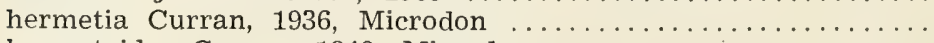

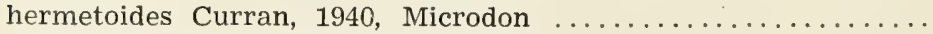

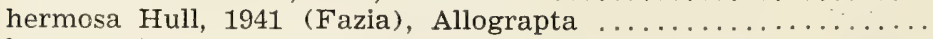

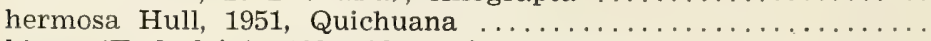

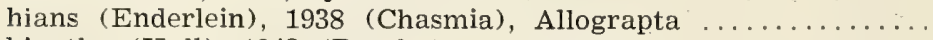

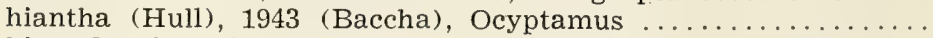
hieroglyphicus (Schiner), 1868 (Mesogramma), Toxomerus ....

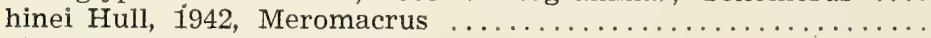

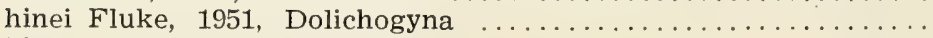
hinei Thompson, Ceriogaster

46.76

46.100

46.90

46.77

46.97

46.31

46.19

46.68

46.77

46.35

46.19

46.50

46.69

46.35

46.70

46.113

46.96

46.121

46.50

46.11

46.65

46.65

46.35

46.98

46.35

46.19

46.51

46.112

46.97

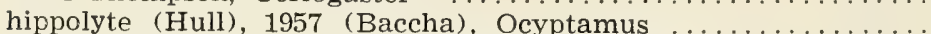
46.116

46.19 hirsuta (Giglio-Tos), 1892 (Volucella), Copestylum

46.73 


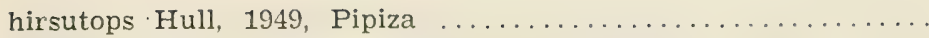

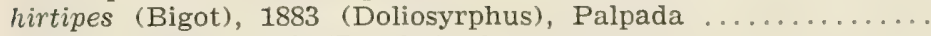

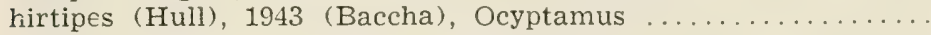
hirtipes (Macquart), 1850 (Volucella), Copestylum ...........

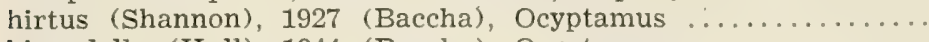

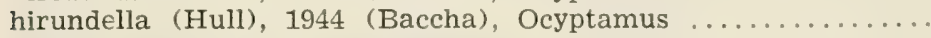
hispida (Giglio-Tos), 1892 (Volucella), Copestylum ..........

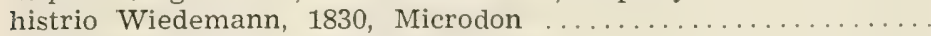
Holmbergia Lynch Arribálzaga, 1891

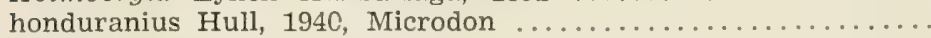

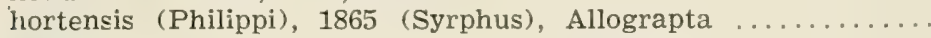
horticole (Hull), 1943 (Volucella), Copestylum ..............

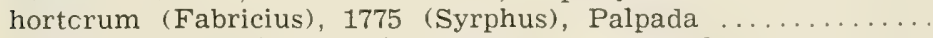
horvathi (Szilády), 1926 (Graptomyza), Copestylum .........

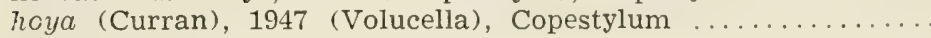

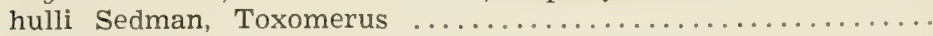

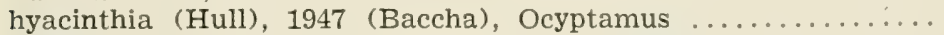
hyalinipenne (Hull), 1944 (Volucella), Copestylum ........... hyalipennis (Curran), 1930 (Callostigma), Ocyptamus ........ hyalcpterum (Giglio-Tos), 1892 (Volucella), Copestylum ......

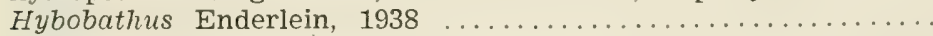
hydrofenestra (Hull), 1944 (Volucella), Copestylum ......... Hypobathus Fluke, 1956

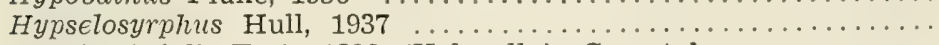
hystrix (Giglio-Tos), 1892 (Volucella), Copestylum ..........

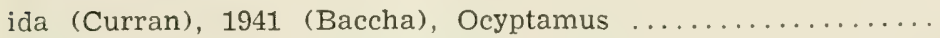

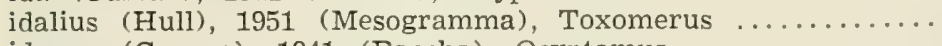

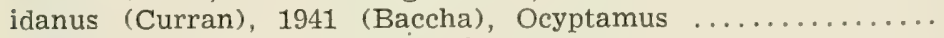

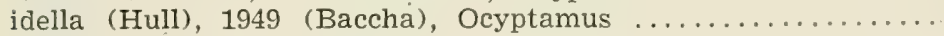

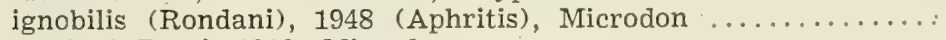

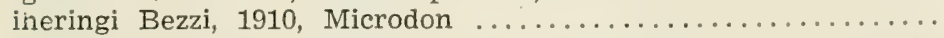

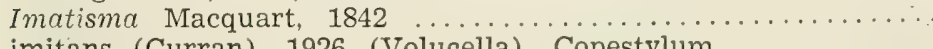
imitans (Curran), 1926 (Volucella), Copestylum ............ imitator (Curran), 1925 (Epistrophe), Allograpta ........... immaculatus (Macquart), 1842 (Syrphus), Ocyptamus ........ imperialis (Curran), 1926 (Mesogramma), Toxomerus ........ impositus (Walker), 1860 (Eristalis), Helophilus ............ impressum (Hull), 1949 (Volucella), Copestylum ...........

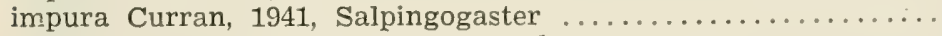

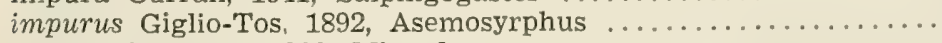

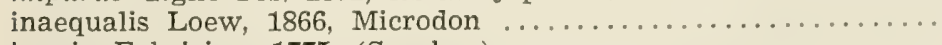

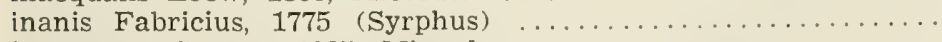

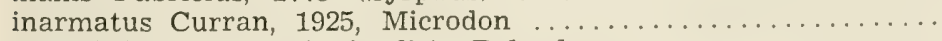

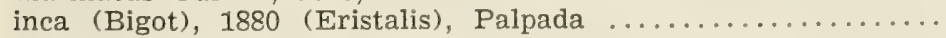

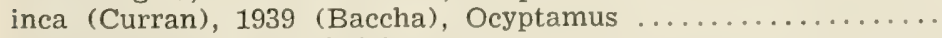

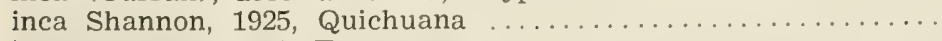

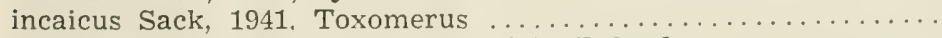

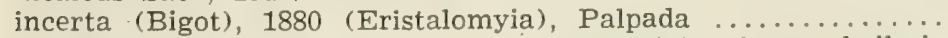
incerta (Shannon \& Aubertin), 1933 (Chilosia), Chromocheilosia incerta Shannon \& Aubertin, 1933, Hemixylota ............

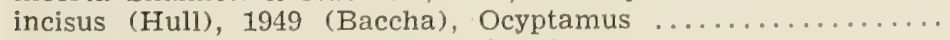

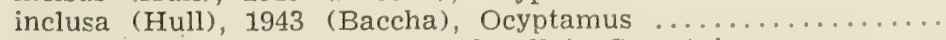
incommodum (Knab), 1916 (Volucella), Copestylum .......... incompta (Austen), 1893 (Baccha), Leucopodella ............ inconsistens (Curran), 1939 (Volucella), Copestylum ..........

46.20

46.51

46.20

46.20

46.65

46.65

46.99

46.77

46.36

46.20

46.51

46.96

46.77

46.32

46.96

46.65

46.39

46.62

46.106

46.20

46.98

46.51

46. 106

46.90

46.121

46.15

46.23

46.77

46.46

46.77 


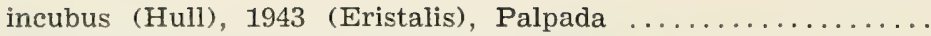

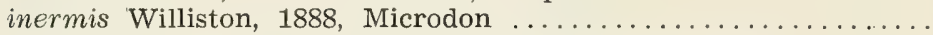

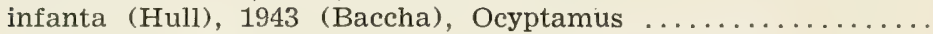
inflatifrons (Fluke), 1945 (Melanostoma), Carposcalis ........

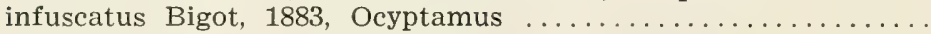

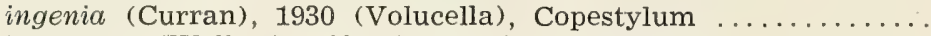

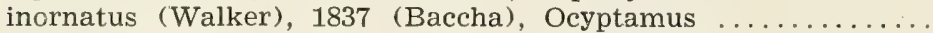

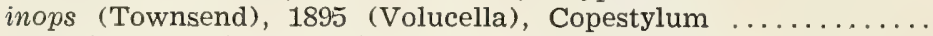

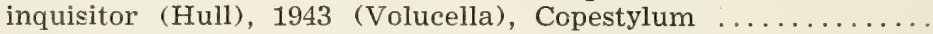
insignis (Schiner), 1868 (Mesogramma), Toxomerus ..........

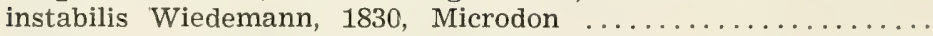

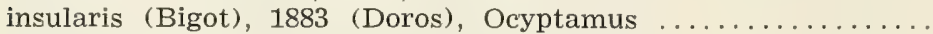

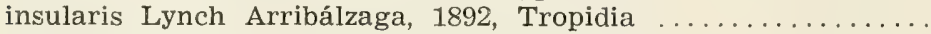

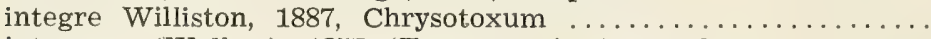
integrum (Walker), 1857 (Temnocera), Copestylum .........

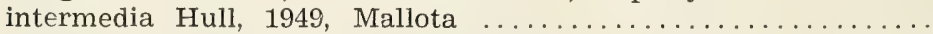
intermedius (Hull), 1949 (Mesogramma), Toxomerus ........

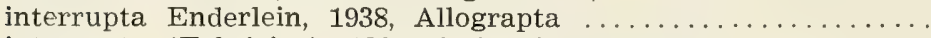

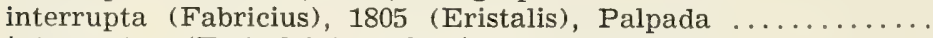
interruptus (Enderlein), 1938 (Mesograpta), Toxomerus .....

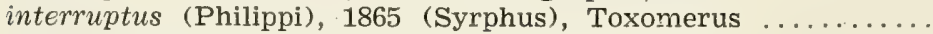

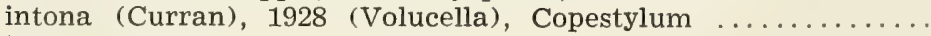

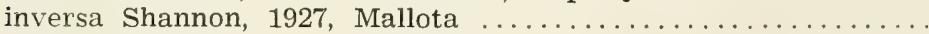

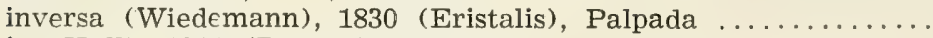

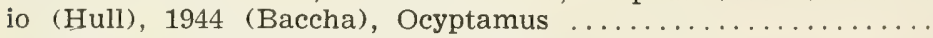

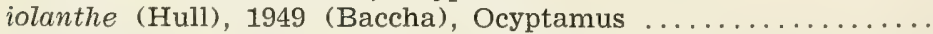

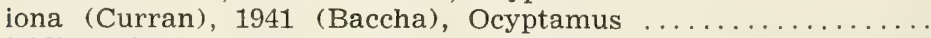

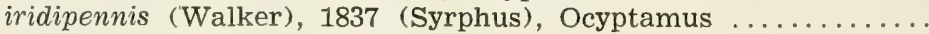

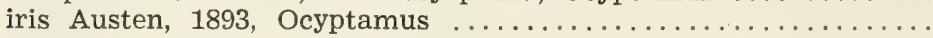
isabellina (Williston), 1887 (Volucella), Copestylum ..........

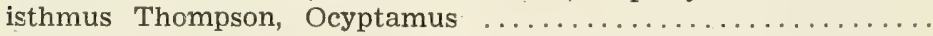

46.106

46.67

46.20

46.41

46.20

46.75

46.20

46.78

46.77

46.51

46.65

46.20

46.120

46.40

46.77

46.100

46.51

46.35

46.106

46.50

46.53

46.77

46.100

46.106

46.20

46.22

46.20

46.18

46.20

46.77

46.20

jactator (Loew), 1861 (Syrphus), Ocyptamus

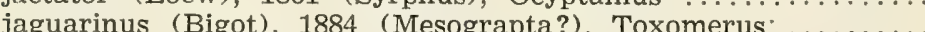

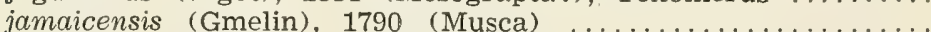
jenningsi (Shannon), 1925 (Lepidostola), Lepidomyia ........

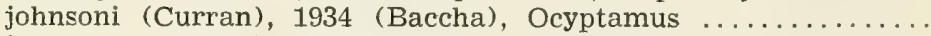
johnsoni (Curran), 1925 (Phalacromyia), Copestylum ........

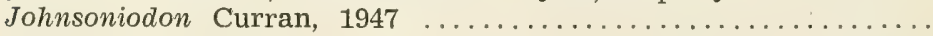

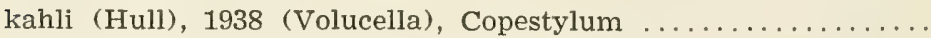

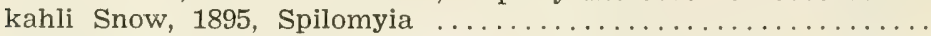

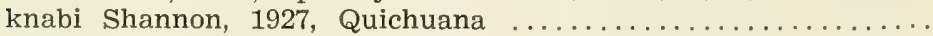
kerteszi Shannon, 1925 (Cerioides), Sphiximorpha ..........

labyrinthops (Hull), 1944 (Chrysogaster), Orthonevra ........

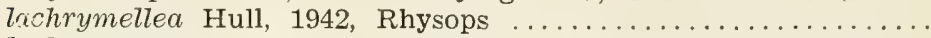

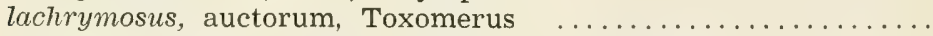
laciniosus (Loew), 1866 (Mesograpta), Toxomerus ...............

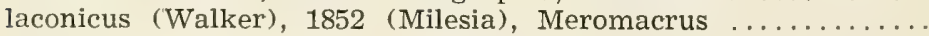

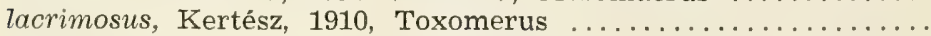
lacrymosus (Bigot), 1884 (Mesograpta?), Toxomerus ........

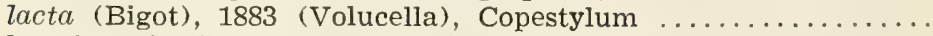

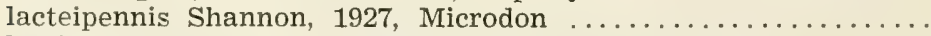
lacticoeruleum (Hull), 1944 (Volucella), Copestylum .........

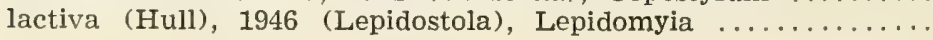

46.20

46.51

46.39

46.88

46.20

46.77

46.61

46.77

46.121

46.99

46.93

46.91

46.43

46.51

46.51

46.112

46.51

46.51

46.78

46.62

46.77

46.89 
laenas Hull, 1943, Toxomerus

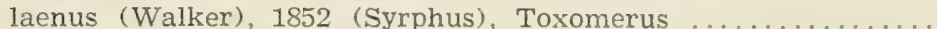

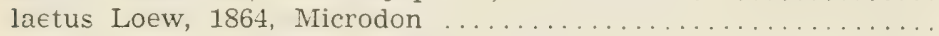

lanei Carrera \& Lenko, 1958, Mixogaster ..................

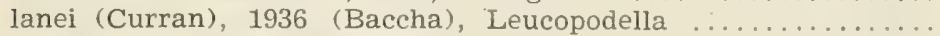
lanei Curran, 1936, Microdon

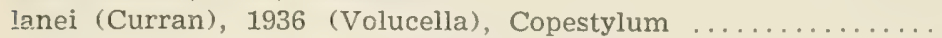

46.46

46.62

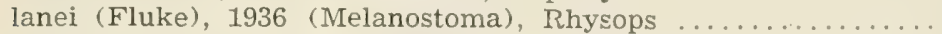

46.77

46.43

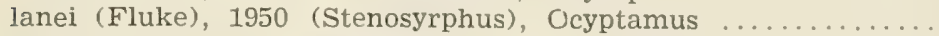

46. 21

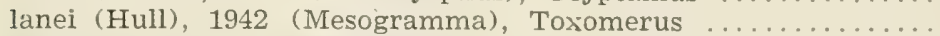

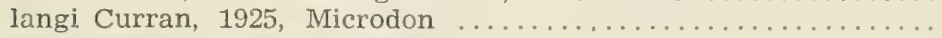

langi (Curran), 1934 (Eristalis), Palpada

langi Curran, 1934, Habromyia

46.51

46.65

46.106

46.100

lasiotibialis Fluke, 1937, Trichopsomyia ...............

46.68

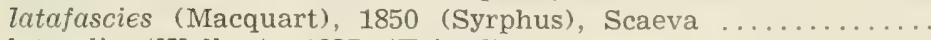

lateralis (Walker), 1837 (Eristalis), Palpada

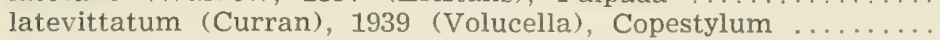

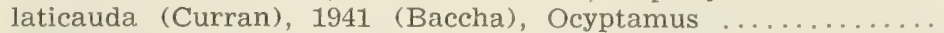

latifacies, Fluke, 1956, Scaeva

46.9

46.104

46.77

46.21

46.9

latifacies (Shannon), 1927 (Claraplumula), Allograpta ......

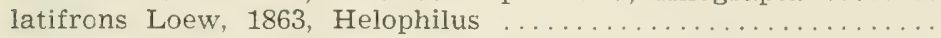

latiforns Loew, 1866, Eristalis

46.36

46.96

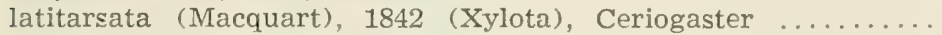

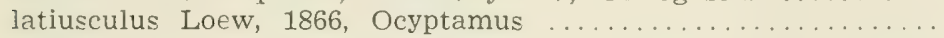

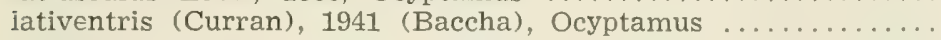

latum (Wiedemann), 1830 (Volucella), Copestylum ..........

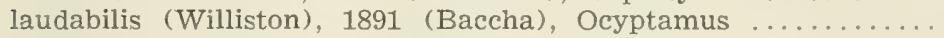

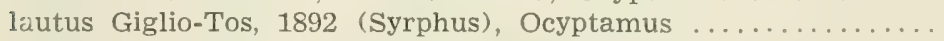

laxus (Osten Sacken), 1875 (Didea), Megasyrphus ...........

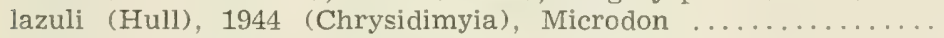

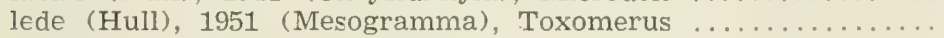

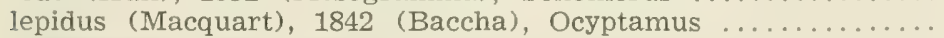

Lepidcmyia Loew, 1864

Lepidopsis Curran, 1925

46.102

46.116

46.21

46.21

46.78

46.21

46.21

46.10

46.65

46.51

46.21

46.88

46.70

46.70

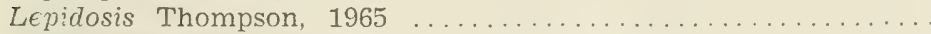

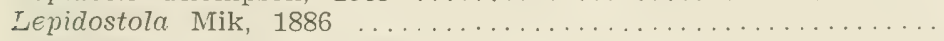

Lepromyia Williston, 1887

46.88

46.88

leucopodus (Hull), 1948 (Baccha), Ocyptamus .............. 46.21

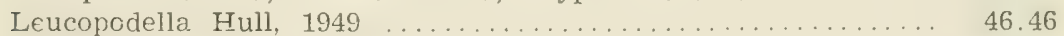

levissimus (Austen), 1893 (Baccha), Ocyptamus ............ 46.21

limae (Brèthes), 1920 (1937) (Mesograpta), Toxomerus ...... 46.51

limbata (Fabricius), 1805 (Scaeva), Allograpta ............ 46.36

limbatinevris (Macquart), 1850 (Eristalis), Palpada ......... 46.109

limbatus Wiedemann, 1830, Microdon ................. 46.65

limpipenne Williston, 1887, Copestylum ................. 46.78

limbipennis Williston, 1891, Salpingogaster ............. 46.32

limbippennis, Sack, 1920, Salpingogaster .............. 46.32

limbus (Enderlein), 1938 (Antiops), Toxomerus ........... 46.51

limpidapex (Curran), 1941 (Baccha), Ocyptamus ........... 46.21

linearis (Wulp), 1883 (Mesograpta), Toxomerus ............ 46.51

lineascripta Huli, 1937. Meromacrus ................ 46.112

lineata (Fabricius), 1805 (Milesia), Senogaster ............ 46.119

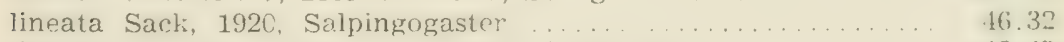

lineatus (Fluke), 1937 (Melanostoma), Rhysops ........... 46.43

lineatus (Macquart), 1846 (Baccha), Ocyptamus .......... 46.21 


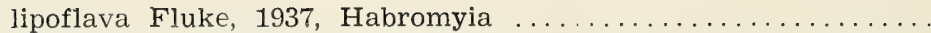

46.100

liposeta Fluke, 1937, Salpingogaster ................ 46.33

liriope (Hull), 1949 (Volucella), Copestylum .............. 46.78

litoralis (Papavero), 1964 (Ubristes), Microdon ............ 46.62

lividus (Schiner), 1868 (Baccha), Ocyptamus .............. 46.21

lizeri (Brèthes), 1914 (Helophilus), Habromyia ............. 46.101

lo€wi (Goot), 1964 (Syrphus), Xanthandrus ............. 46.44

loewi (Sedman), 1965 (Baccha), Ocyptamus .............. 46.27

loewi (Williston), 1892 (Pteroptila), Meromacrus .......... 46.113

longicornis Loew, 1872, Spilomyia .................... 46.121

longicornis (Walker), 1837 (Pipiza), Rhysops .............. 46.43

longicornis (Wiedemann), 1824 (Ceratophya), Microdon ...... 46.59 .

longicornis (Williston), 1888 (Melanostoma), Rhysops ....... 46.43

longicornis Williston, 1888, Trichopsomyia ............. 46.68

longipenis (Enderlein), 1938 (Posthonia), Eupeodes ........ 46.9

longirostris Fluke, 1943, Rhingia ................... 46.69

lopesae (Curran), 1941 (Baccha), Ocyptamus ............ 46.18

lopesi Carrera \& Lenko, 1958, Mixogaster ................ 46.58

Iopesi Fluke, 1945, Rhysops ................... 46.43

lotus (Williston), 1887 (Syrphus), Dasysyrphus ............. 46.10

lucana (Townsend), 1895 (Volucella), Copestylum .......... 46.78

lucasana (Townsend), 1895 (Volucella), Copestylum ......... 46.78

lucifera (Hull), 1943 (Rhinoprosopa), Allograpta .......... 46.36

lucilia (Hull), 1950 (Volucella), Copestylum ............. 46.78

lucretia (Hull), 1949 (Baccha), Ocyptamus .............. 46.21

luctuosa (Bigot), 1857 (Helophilus), Myolepta ............. 46.90

luctuosa (Philippi), 1865 (Priomerus), Myolepta .......... 46.90

luctuosus (Bigot), 1883 (Baccha), Ocyptamus ............. 46.21

luculentus (Fluke), 1945 (Melanostoma); Rhysops ......... 46.43

lugens (Wiedemann), 1830 (Volucella), Copestylum ......... 46.78

lugubri (Jaennicke), 1867 (Chrysogaster), Orthonevra ....... 46.91

Iugubris (Philippi), 1865 (Baccha), Ocyptamus ............ 46.21

lugubris (Williston), 1891 (Baccha), Ocyptamus .......... 46.22

lumina (Hull), 1937 (Volucella), Copestylum ............. 46.78

luna (Fluke), 1942 (Epistrophe), Allograpta ............. 46.36

lundbladi Enderlein, 1940, Carposcalis ................. 46.41

lunuliferum (Hull), 1937 (Volucella), Copestylum .......... 46.78

lunus (Hull), 1943 (Mesogramma), Toxomerus ............. 46.51

luridescens (Walker), 1857 (Ceratophya), Microdon ......... 46.65

lutescens (Walker), 1857 (Rhoga), Microdon ............ 46.60

lutzi (Curran), 1930 (Mesogramma), Toxomerus ........... 46.51

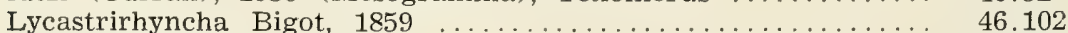

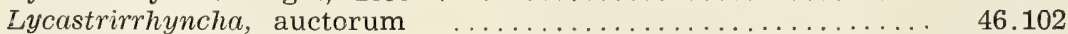

Lycopale Hull, 1944 ............................... 46.100

lyncharribalzagai Shannon, 1927, Polybiomyia ........... 46.95

lynchii (Williston), 1888 (Ceria), Monoceromyia .......... 46.94

lyratus (Hull), 1940 (Mesogramma), Toxomerus ........... 46.52

lyrica (Curran), 1941 (Planes), Neplas ................... 46.115

macer (Curran), 1930 (Baccha), Ocyptamus ............ 46.22

mackiei Curran, 1940, Microdon ...................... 46.62

macquarti (Blanchard), 1852 (Syrphus), Allograpta ......... 46.36

macquarti (Curran), 1926 (Volucella), Copestylum .......... 46.78

macquarti (Goot), 1964 (Mesograpta), Toxomerus ........... 46.52

macquarti Shannon, 1925, Polybiomyia ............... 46.95 
macquartii Lynch Arribálzaga, 1891, Microdon ............ macra (Enderlein), 1938 (Desmetrum), Chamaesphegina ...... macrocephalum (Giglio-Tos), 1892 (Camerania), Copestylum Macrometopia Phillippi, 1865

macroptera (Curran), 1941 (Ceratophya), Argentinomyia .......

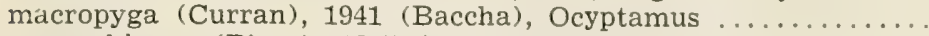
macrorhinum (Bigot), 1875 (Volucella), Copestylum .........

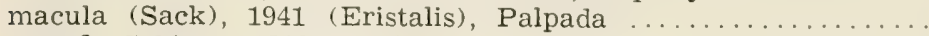

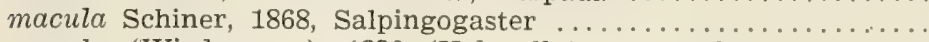

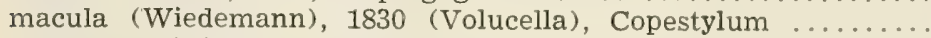
maculatus (Bigot), 1884 (Mesograpta?), Toxomerus ........... maculatus (Macquart), 1850 (Milesia), Meromacrus ..........

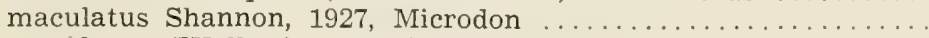

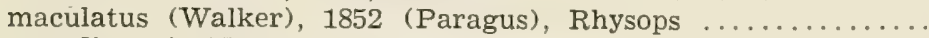

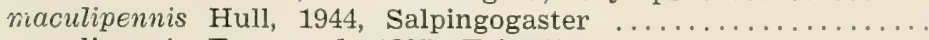

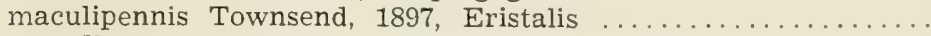
maculipes (Bigot), 1884 (Mesograpta?), Toxomerus ........ maculoides (Curran), 1939 (Volucella), Copestylum ........... magnifica (Bigot), 1880 (Plagiocera), Habromyia ............. major Curran, 1930, Ornidia

malleri (Curran), 1947 (Johnsoniodon), Microdon ..........

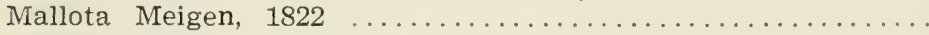

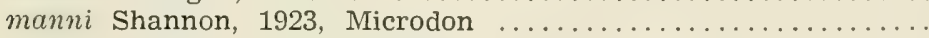

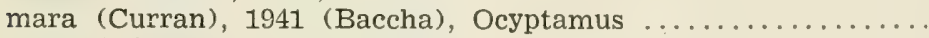
marceli Curran, 1936, Microdon marceli (Curran), 1939 (Volucella), Copestylum ............

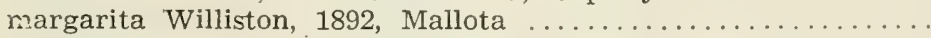
marginatum (Say), 1830 (Volucella), Copestylum ..........

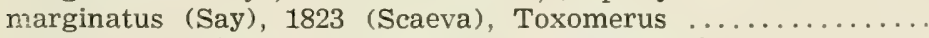

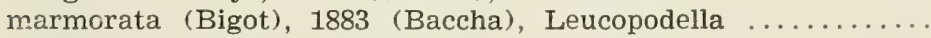

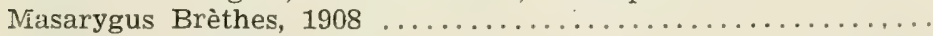

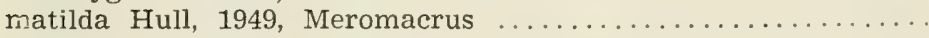
maximiliani (Jaennicke), 1867 (Volucella), Copestylum .......

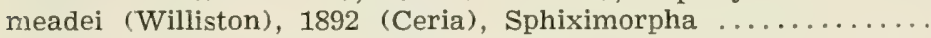
megacephalum (Loew), 1863 (Tomnocera), Copestylum .......

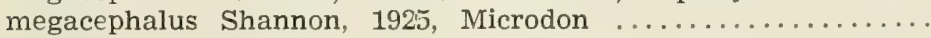
Megametopon Giglio-Tos, 1891

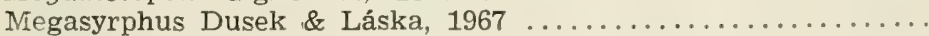
meigenii (Wiedemann), 1830 (Eristalis), Palpada ........... melanaspis (Wiedemann), 1830 (Eristalis), Palpada ........... melanocera (Williston), 1891 (Melanostoma), Rhysops ......... melanogaster (Thomson), 1869 (Syrphus), Toxomerus ........ melanoptera (Barretto \& Lane), 1947 (Eurypterosyrphus), Aristosyrphus

melanorhina (Bigot), 1883 (Phalacromyia), Copestylum ..... melanorrhinus (Philippi), 1865 (Baccha), Ocyptamus .........

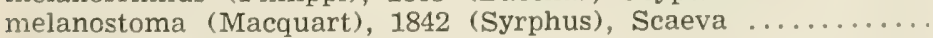
Mielanostoma Schiner, 1860

Nelanostomatini

MIelithreptus Loew, 1840

Melitropus Haliday, 1856

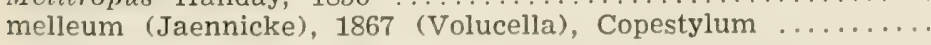
melleus Curran, 1940, Microdon melleoguttatus (Hull), 1941 (Mesogramma), Toxomerus ......

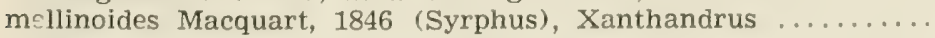
mellinum (Linnaeus), 1758 (Musca), Melanostoma

46.65

46.91

46.78

46.116

46.57

46.22

46.78

46.106

46.33

46.78

46.52

46.111

46.60

46.43

46.31

46.102

46.49

46.78

46.101

46.69

46.61

46.99

46.66

46.22

46.66

46.78

46.100

46.78

46.52

46.47

46.60

46.112

46.79

46.93

46.78

46.62

46.70

46.10

46.107

46.107

46.43

46.52

46.58

46.85

46.22

46.9

46.42

46.40

46.38

46.38

46.79

46.60

46.53

46.45

46.42 


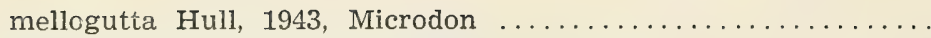

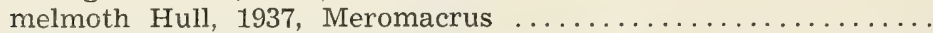

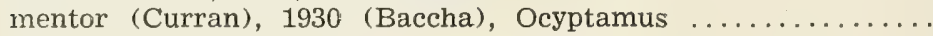

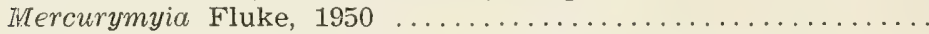
meretricias (Williston), 1888 (Volucella), Copestylum ........ meridionalis Fluke, 1950 (Sternosyrphus), Ocyptamus ....... meridionalis (Goot), 1964 (Syrphus), Ocyptamus ............

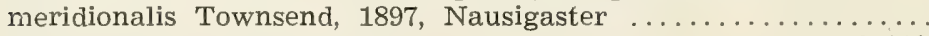
Meromacrus Rondani, $1848 \ldots \ldots \ldots \ldots \ldots \ldots \ldots \ldots$ 46.110, Mesogramma Loew, 1866 Mesograpta Loew, 1872 metallica (Bigot), 1882 (Eurhinomallota), Criorhina .......... metalliferum (Walker), 1849 (Volucella), Copestylum ......... metalliferum (Walker), 1849 (Volucella), Copestylum ........

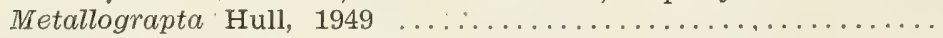
metallorum (Walker), 1852 (Temnocera), Copestylum .........

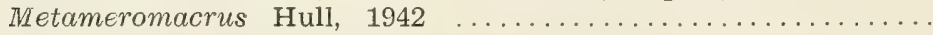

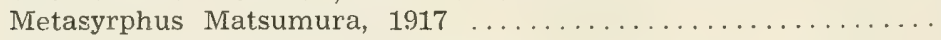
Metepistrophe Hull, 1949

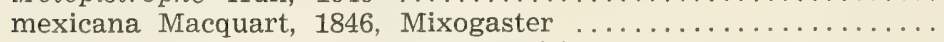

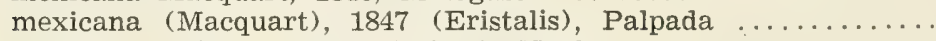

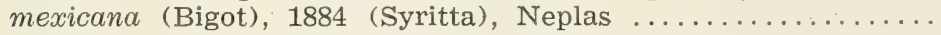

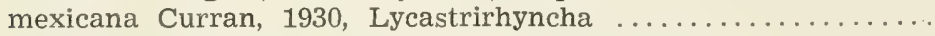

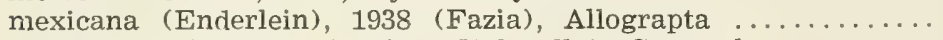
mexicanum (Macquart), 1842 (Volucella), Copestylum ........ mexicanus (Curran), 1930 (Baccha), Ocyptamus .............

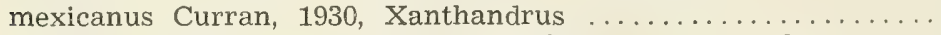
mexicanus (Macquart), 1842 (Helophilus), Asemosyrphus .....

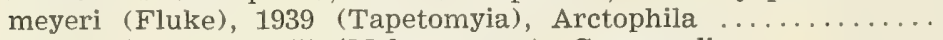
meyeri (Fluke), 1945 (Melanostoma), Carposcalis ........... Microdon Meigen, $1803 \ldots \ldots \ldots \ldots \ldots \ldots \ldots \ldots \ldots$ 46.59, Microdontinae

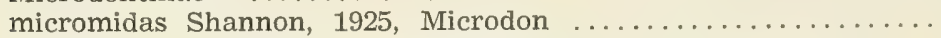
micropelecinus (Shannon), 1927 (Baccha), Ocyptamus ........

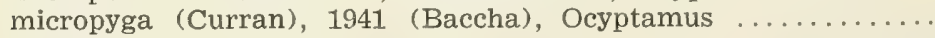

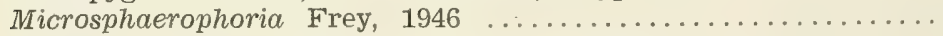
micrura (Osten Sacken), 1877 (Sphaerophoria), Allograpta ...

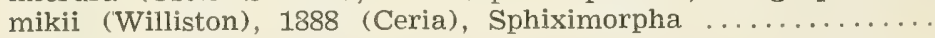

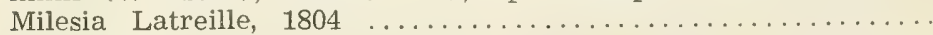

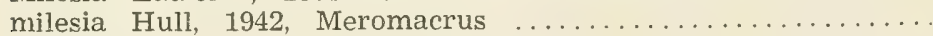

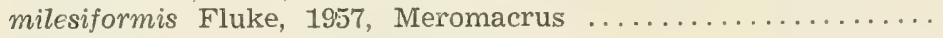
Milesiinae

Milesiini

milesioides (Bigot), 1880 (Eristalomyia), Meromacrus ......... millesiformis (Macquart), 1834 (Mallota), Meromacrus ........

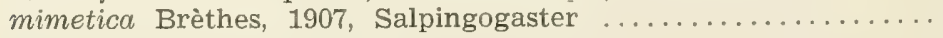
mimima, Hull, 1949, Ocyptamus . . . . . . . . . . . . . .

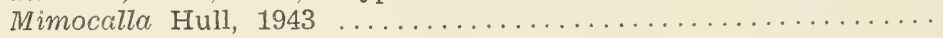

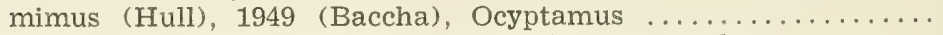
minimum (Giglio-Tos), 1892 (Volucella), Copestylum .........

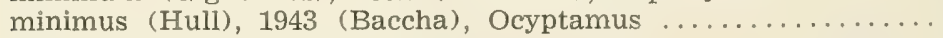

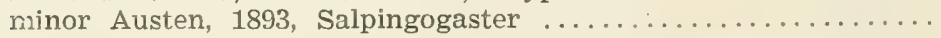

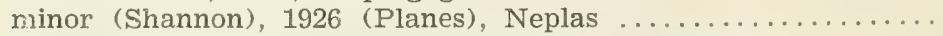
minotaurus (Speiser), 1914 (Nosodepus), Dolichogyna ........

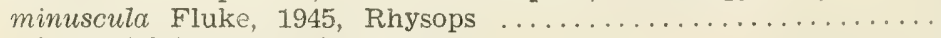
minuta Fluke, 1956, Myolepta 
minutalis (Williston), 1891 (Eristalis), Palpada ............

46.107

minutulus (Doesburg), 1966 (Cerathophya), Paragodon ........

46.57

minutus (Wiedemann), 1830 (Syrphus), Toxomerus ..........

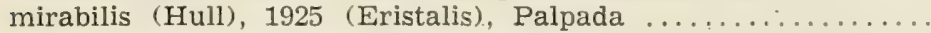

mirabilis Williston, 1888, Microdon

46.52

46.107

46.66

nissionera (Lynch Arribálzaga), 1892 (Volucella), Copestylum

46.79

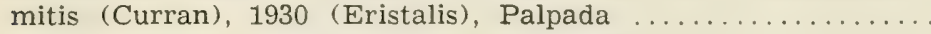

46.107

mitis (Curran), 1930 (Mesogramma), Toxomerus .......... 46.52

mitis Curran, 1940, Microdon ..................... 46.66

Míitrosphen Enderlein, 1938 ........................ 46.47

Mixcgaster Macquart, 1842 ....................... 46.58

mixtus Curran, 1940, Microdon .................... 46.62

mocanum (Curran), 1936 (Volucella), Copestylum ......... 46.79

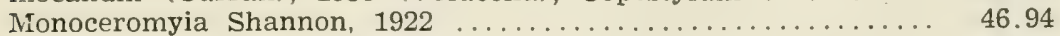

montana Hull, 1951, Quichuana ........................ 46.99

montana (Roeder), 1886 (Eristalis), Palpada .............. 46.107

montensis Hull, 1938 (Nosodepus) .................... 46.122

monticola (Roeder), 1892 (Eristalis), Palpada ............... 46.107

morpho (Curran), 1939 (Volucella), Copestylum .......... 46.79

mu (Bigot), 1884 (Mesograpta?), Allograpta ............. 46.36

mulio (Hull), 1941 (Mesogramma), Toxomerus ............ 46.52

mulleri Fluke, 1951, Dolichogyna ................... 46.97

multipunctatus (Wulp), 1883 (Mesograpta), Toxomerus ..... 46.52

munozi Enderlein, 1938, Allograpta ................. 46.36

murinus (Curran), 1930 (Baccha), Ocyptamus ........... 46.22

mus Curran, 1936, Microdon .......................... 46.66

mus (Curran), 1930 (Eristalis), Palpada ................. 46.109

mus (Williston), 1888 (Volucella), Copestylum ............ 46.79

musanum (Curran), 1930 (Volucella), Copestylum .......... 46.79

muscarium (Thomson), 1869 (Haurotricha), Copestylum ..... 46.79

musicanum (Curran), 1930 (Volucella), Copestylum ........ 46.79

musicus (Fabricius), 1805 (Scaeva), Toxomerus ............ 46.52

miusta (Williston), 1888 (Volucella), Copestylum ............ 46.86

mustoides (Curran), 1927 (Volucella), Copestylum .......... 46.79

mutuus (Say), 1829 (Syrphus), Toxomerus ............... 46.52

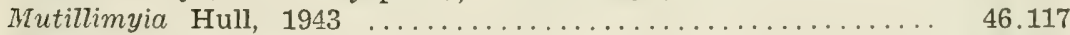

Myiolepta, auctorum ....................... 46.89

Myolepta Newman, 1838 ....................... 46.89

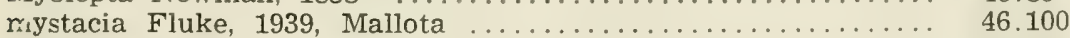

myrtella (Hull), 1960 (Baccha), Ocyptamus .............. 46.22

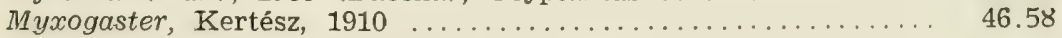

Nannomyrmecomyia Hull, $1945 \ldots \ldots \ldots \ldots \ldots \ldots \ldots \ldots . \ldots \ldots . \ldots \ldots$

nasicum (Williston), 1891 (Ophromyia), Copestylum ........ 46.79

nasicus (Hull), 1941 (Mesogramma), Toxomerus ........... 46.52

nasigera (Enderlein), 1938 (Fazia), Allograpta ........... 46.36

nasuta (Bigot), 1884 (Sphaerophoria), Allograpta ......... 46.36

nasuta (Bigot), 1888 (Sphaerophoria), Allograpta ......... 46.36

nasuta (Enderlein), 1938 (Chasmia), Allograpta ........... 46.37

nasutus Sack, 1941, Toxomerus .................. 46.52

nasutus (Williston), 1891 (Baccha), Ocyptamus ........... 46.22

nausicaa (Hull), 1937 (Nausigaster), Myolepta ............ 46.90

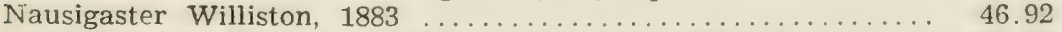

nautlanum (Townsend), 1897 (Volucella), Copestylum ........ 46.80

nectarinoides (Lynch Arribálzaga), 1892 (Promilesia), Meromacrus 46.112 


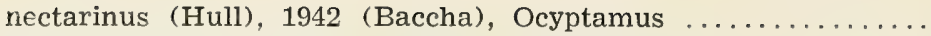

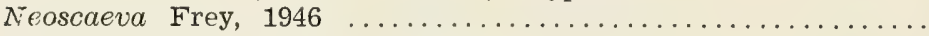

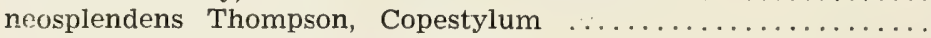

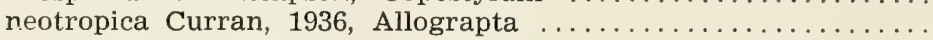

neotropica (Shannon), 1925 (Chrysogaster), Orthonevra ......

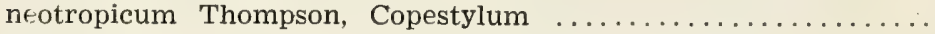

neotropicus (Curran), 1937 (Melanostoma), Rhysops ..........

neotropicus (Hull), 1943 (Mesogramma), Toxomerus ..........

Neplas Porter, 1927

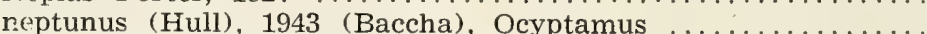

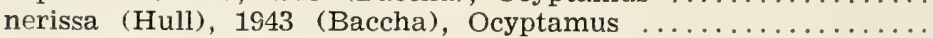

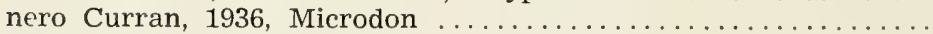

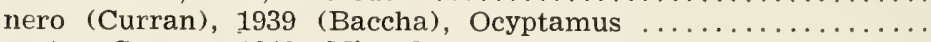

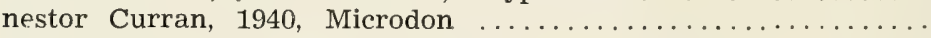

neuralis (Curran), 1934 (Callostigma), Ocyptamus ..........

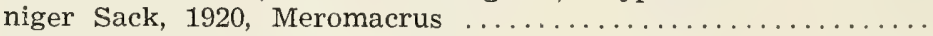

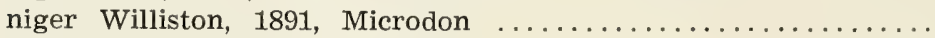

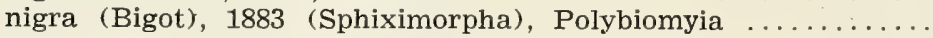

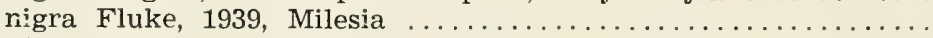

nigra (Giglio-Tos), 1892 (Platynochaetus), Quichuana .........

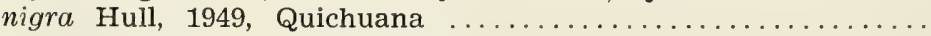

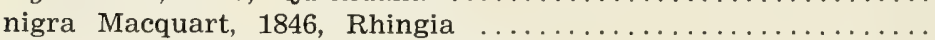

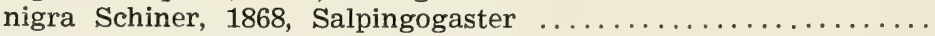

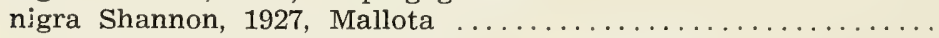

nigra (Shannon), 1927 (Valdivia), Valdiviomyia ............

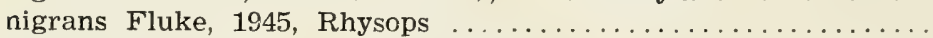

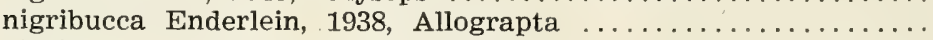

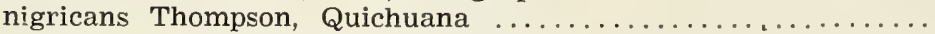

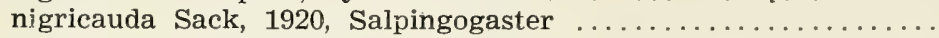

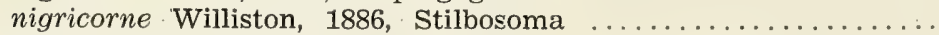

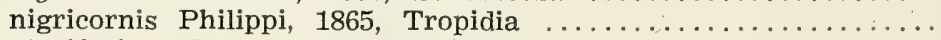

nigrifacies (Bigot), 1875 (Volucella), Copestylum ............

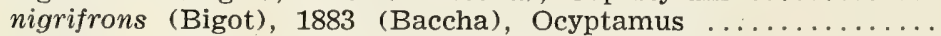

nigrifrons (Hine), 1914 (Phalacromyia), Copestylum ..........

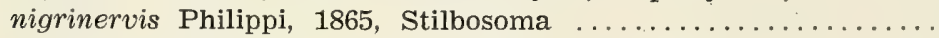

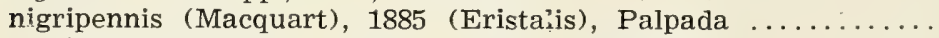

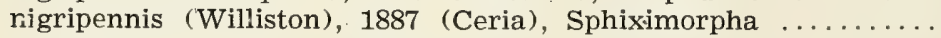

nigripes (Bigot), 1857 (Phalacromyia), Copestylum .........

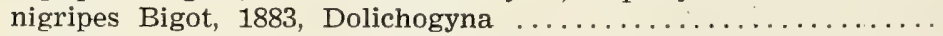

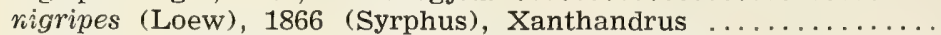

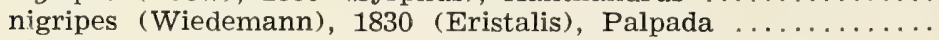

nigripunctus (Enderlein), 1938 (Mesogramma), Toxomerus ... nigriscutellata (Macquart), 1848 (Eristalis), Palpada .........

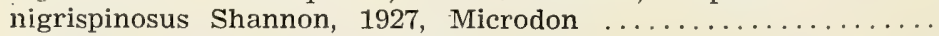

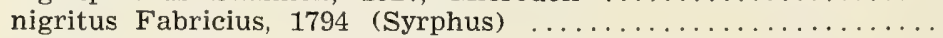

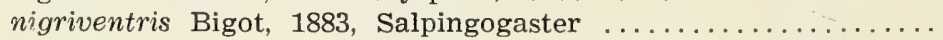

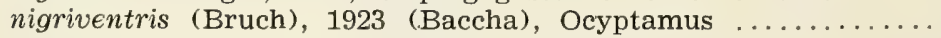

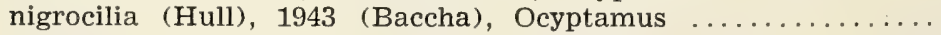

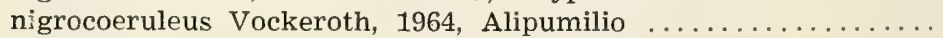
nigropodum (Hull), 1949 (Volucella), Copestylum ...........

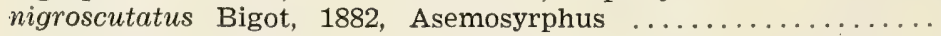

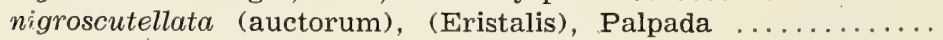
nigroventris, Williston, 1886, Salpingogaster $\ldots \ldots \ldots \ldots \ldots \ldots$ nigroviride (Hull), 1949 (Volucella), Copestylum ........... nigrum (Greene), 1923 (Volucella, Copestylum ............. 


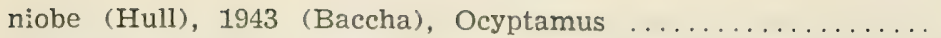

nitens Bigot, 1859, Lycastrirhyncha

46.23

nitescens (Shannon \& Aubertin), 1933 (Chilosia), Notiocheilosia nitida (Wiedemann), 1830 (Chrysogaster), Orthonevra

ritida Sack, 1941 (Cerioides)

nitidigaster (Hull), 1937 (Volucella), Copestylum ............

nitidiventris (Curran), 1930 (Mesogramma), Toxomerus .....

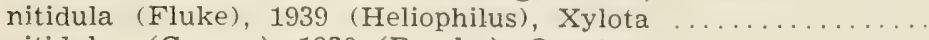

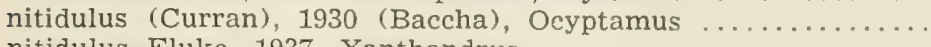

nitidulus Fluke, 1937, Xanthandrus

46.102

46.88

46.91

46.95

46.80

46.53

46.114

46.23

ritidus (Schiner), 1868 (Mesogramma), Toxomerus .......... 46.53

nodosus (Hull), 1930 (Baccha), Ocyptamus ............. 46.23

nora (Curran), 1941 (Baccha), Ocyptamus ............. 46.23

norina (Curran), 1941 (Baccha), Ocyptamus ............. 46.23

norma (Curran), 1930 (Mesogramma), Toxomerus .......... 46.53

normalis Curran, 1925, Microdon ................... 46.66

Nosodepus Speiser, 1914 ........................ 46.97

notata (Bigot), 1882 (Ortholophus), Tropidia ............ 46.120

notata Doesburg, 1966, Allograpta ................ 46.34

notatum (Bigot), 1875 (Volucella), Copestylum ............ 46.80

notatus Coquillett, 1902, Ocyptamus ................... 46.19

notatus (Loew), 1866 (Baccha), Ocyptamus .............. 46.23

notatus (Wiedemann), 1824 (Ceratophya), Microdon ........ 46.59

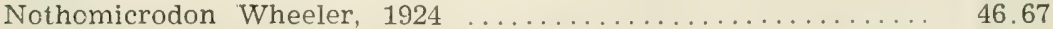

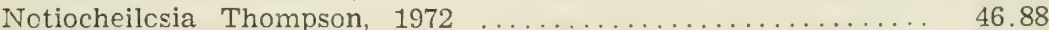

Notosyrphus Vockeroth, 1969 ..................... 46.10

nova Giglio-Tos, 1892, Salpingogaster ................. 46.32

novum (Fluke), 1951 (Volucella), Copestylum ............. 46.84

nubeculosus Wulp, 1888 (Pterallastes) ................ 46.122

nvmphaea (Hull), 1943 (Baccha), Ocyptamus ............ 46.23

nymphalius (Hull), 1942 (Mesogramma), Toxomerus ....... 46.53

ohesa (Fabricius), 1775 (Syrphus), Ornidia ............. 46.69

obesoides (Giglio_Tos), 1892 (Volucella), Ornidia .......... 46.70

obligua (Curran), 1940 (Eristalis), Palpada .............. 46.107

obliqua (Say), 1823 (Scaeva), Allograpta ............... 46.36

obliquicorne (Curran), 1939 (Volucella), Copestylum ........ 46.80

obliquus (Curran), 1941 (Callostigma), Ocyptamus ......... 46.23

oblongus (Walker), 1852 (Baccha), Ocyptamus ........... 46.23

obscura Coquillett, 1902, Spilomyia ................... 46.121

obscuripenne (Lynch Arribálzaga), 1892 (Volucella), Copestylum 46.80

cbscurius (Curran), 1939 (Volucella), Copestylum .......... 46.80

obscurus Hine, 1924, Meromacrus ..................... 46.112

obsoleta (Wiedemann), 1830 (Eristalis), Palpada ........... 46.107

obsoletus (Curran), 1941 (Baccha), Ocyptamus ............ 46.23

occidentalis Shannon, 1927, Scaeva ................. 46.9

ochracea (Hull), 1941 (Edwardsietta), Habromyia ........... 46.101

ochracea (Williston), 1888 (Eristalis), Palpada ............ 46.107

ochraceus (Hull), 1942 (Mesogramma), Toxomerus .......... 46.53

ochreolinea (Hull), 1944 (Baccha), Ocyptamus ............. 46.23

ochrogaster (Thomson), 1869 (Syrphus), Toxomerus ......... 46.50

octoguttatus (Jaennicke), 1867 (Syrphus), Toxomerus ....... 46.53

octomaculata Enderlein, 1938, Allograptina .............. 46.39

cctomaculatus Thompson, Ocyptamus .................. 46.24

octomaculatus Walker, 1837, Syrphus ................. 46.8 


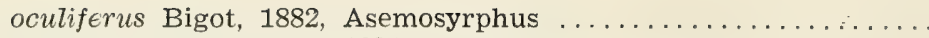

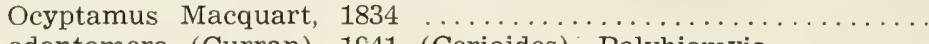

odontomera (Curran), 1941 (Cerioides), Polybiomyia ..........

odyneroides (Philippi), 1865 (Doros), Odyneromyia

46.11

Odyneromyia Shannon \& Aubertin, $1933 \ldots \ldots \ldots \ldots \ldots \ldots \ldots$

46.95

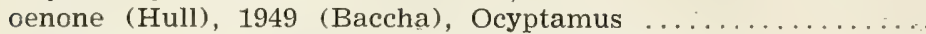

46. 117

46.117

46.24

oestroides (Hull), 1943 (Volucella), Copestylum ........... 46.80

olga (Hull), 1942 (Baccha), Leucopodella ................. 46.47

oligonax Hull, 1944, Microdon ...................... 46.66

Oligorhina Hull, $1937 \ldots \ldots \ldots \ldots \ldots \ldots \ldots \ldots \ldots \ldots \ldots . \ldots \ldots . \ldots \ldots$

olivaceus Giglio-Tos, 1892, Asemosyrphus .............. 46.96

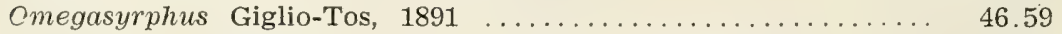

omochroma (Giglio-Tos), 1892 (Volucella), Copestylum ....... 46.80

opacus Fluke, 1945, Rhysops …................... 46.44

opacus Fluke, 1950 (Stenosyrphus), Ocyptamus ........... 46.24

opalicolor (Hull), 1943 (Volucella), Copestylum ............. 46.80

opalina (Townsend), 1897 (Volucella), Copestylum .......... 46.84

opeostoma (Hull), 1949 (Volucella), Copestylum .......... 46.80

ophiolinea (Hull), 1943 (Baccha), Toxomerus .............. 46.53

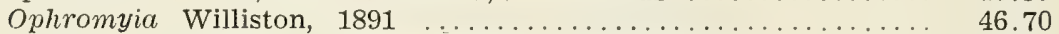

opinator (Williston), 1891.(Volucella), Copestylum ........ 46.80

opulentus (Bigot), 1883 (Eristalis), Meromacrus ............ 46.112

cpulentus Bigot, 1883, Microdon .................. 64.66

criel (Hull), 1942 (Baccha), Ocyptamus ............... 46.24

ornata (Townsend), 1897 (Eritalis), Palpada ............ 46.108

ornatipes (Curran), 1927 (Baccha), Ocyptamus ........... 46.24

ornatum (Williston), 1891 (Volucella), Copestylum .......... 46.80

ornatus (Hull), 1943 (Mesogramma), Toxomerus ........... 46.51

ornatus Sack, 1941, Toxomerus ................. 46.53

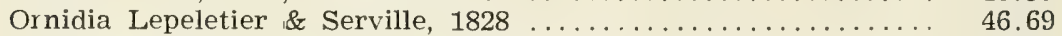

ornithoglyphus (Hull), 1942 (Mesogramma), Toxomerus ...... 46.53

orpheus Hull, 1944, Mixogaster ................... 46.58

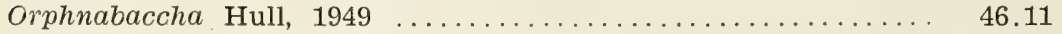

ortalina Wulp, 1888, Lepidomyia .................... 46.89

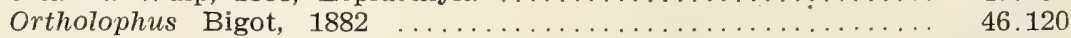

Orihoneura, auctorum ........................... 46.90

Orthonevra Macquart, 1829 ......................... 46.90

osburni (Hull), 1942 (Volucella), Copestylum ............ 46.80

ovatus (Hull), 1942 (Mesogramma), Toxomerus .......... 46.53

oviphorus (Hull), 1943 (Baccha), Ocyptamus ........... 46.24

ovipositorius (Hull), 1943 (Baccha), Ocyptamus .......... 46.24

pachecoi (Curran), 1939 (Volucella), Copestylum .......... 46.81

pachypyga Bigot, 1884, Sphaerophoria ................ 46.38

pachymera (Loew), 1866 (Xylota), Neplas .............. 46.115

pachypoda (Bigot), 1880 (Eristalomyia), Palpada .......... 46.107

pachypus (Wiedemann), 1830 (Eristalis), Meromacrus ....... 46.112

palitarsis (Curran), 1934 (Planes), Neplas ............... 46.115

pallens (Wiedemann), 1830 (Volucella), Copestylum ........ 46.81

palliatus (Fluke), 1945 (Melanostoma), Xanthandrus ....... 46.45

pallidithorax (Hull), 1941 (Volucella), Copestylum .......... 46.81

pallidius (Giglio-Tos), 1892 (Volucella), Copestylum ......... 46.82

pallidum (Macquart), 1842 (Volucella), Copestylum ........ 46.81

pallipes (Bigot), 1883 (Spilomyia), Blera ............... 46.119

pallipes (Bigot), 1884 (Mesograpta?), Toxomerus .......... 46.55 
pallipes Bigot, 1884, Syrphus

46.8

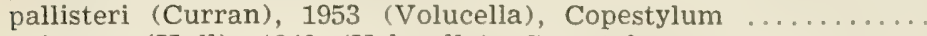

46.81

palmyra (Hull), 1949 (Volucella), Copestylum .............

Palpada Macquart, 1834

46.81

panamana Hull, 1944, Salpingogaster

46.102

46.32

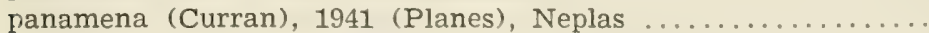

46.115

panamense (Curran), 1930 (Volucella), Copestylum ......... 46.81

panamensis (Curran), 1930 (Baccha), Ocyptamus ......... 46.24

panamensis (Curran), 1930 (Callostigma), Ocyptamus ....... 46.20

panamensis Curran, 1930, Ceriogaster .................... 46.116

nanamensis (Curran), 1930 (Mesogramma), Toxomerus ...... 46.53

panamensis Curran, 1930, Meromacrus ............... 46.113

panamensis Curran, 1930, Microdon .................. 46.59

panamenum (Curran), 1939 (Volucella), Copestylum ....... 46.81

Pandasyopthalmus Stuckenberg, $1954 \ldots \ldots \ldots \ldots \ldots \ldots \ldots . . \ldots . . . . .46 .40$

pandora (Hull), 1942 (Baccha), Ocyptamus ............... 46.24

pannamana, Fluke, 1957, Salpingogaster ............... 46.32

papilio (Hull), 1942 (Baccha), Ocyptamus .............. 46.24

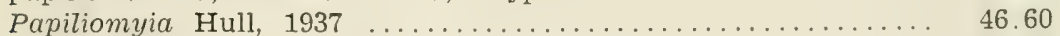

papilionarius (Hull), 1943 (Baccha), Ocyptamus ........... 46.24

para (Curran), 1941 (Baccha), Ocyptamus ................ 46.24

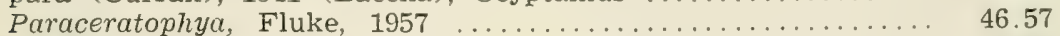

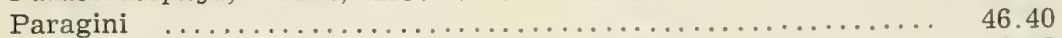

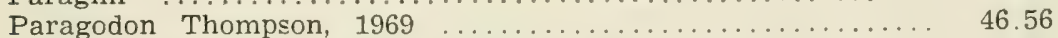

paragoides Thompson, 1969, Paragodon ................. 46.57

paragrammus (Schiner), 1868 (Mesogramma), Toxomerus .... 46.53

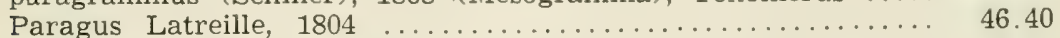

Paramicrodon Meijere, $1913 \ldots \ldots \ldots \ldots \ldots \ldots \ldots \ldots \ldots \ldots \ldots . \ldots \ldots . \ldots \ldots$

parana (Hull), 1942 (Volucella), Copestylum ............. 46.81

parina (Fluke), 1951 (Volucella), Copestylum ............ 46.81

parisii Ceresa, 1934, Quichuana .................... 46.99

parvicornis (Loew), 1861 (Baccha), Ocyptamus ............ 46.24

parvula (Williston), 1888 (Eristalis), Palpada .............. 46.108

parvum Giglio-Tos, 1892, Copestylum ................ 46.84

parvum (Rondani), 1848 (Volucella), Copestylum ........... 46.81

patagonus Lynch Arribálzaga, 1892, Syrphus ............. 46.8

pauper (Rondani), 1848 (Aphritis), Microdon ................. 46.66

pauxilla (Williston), 1892 (Xylota), Neplas ............. 46.115

pectinis Fluke, 1943, Tuberculanostoma ............... 46.45

pectorale (Rondani), 1863 (Volucella), Copestylum .......... 46.81

pedicellata (Williston), 1887 (Ceria), Polybiomyia ........... 46.95

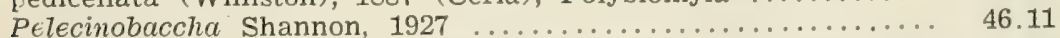

penaltis (Curran), 1934 (Eristalis), Palpada .............. 46.108

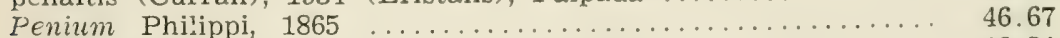

pennatus (Hull), 1943 (Baccha), Ocyptamus ............. 46.24

pennipes (Sack), 1941 (Tubifera), Dolichogyna ............ 46.98

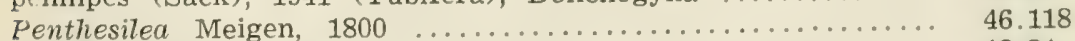

peri (Hull), 1943 (Baccha), Ocyptamus ................ 46.24

perlatum (Hine), 1914 (Volucella), Copestylum ........... 46.81

perpolita (Johnson), 1921 (Lepidostola), Lepidomyia ......... 46.89

persa Williston, 1891, Eristalis .................... 46.102

persimile (Williston), 1888 (Volucella), Copestylum ......... 46.81

persimilis (Curran), 1930 (Baccha), Ocyptamus ............ 46.24

pertinax (Hull), 1950 (Volucella), Copestylum ............. 46.81

peruana Sack, 1941, Dolichogyna ................. 46.97 
peruvianum (Vimmer \& Soukop), 1938 (Volucella), Copestylum peruvianus (Shannon), 1927 (Baccha), Ocyptamus ..........

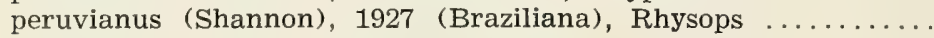

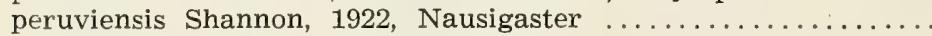
pessulagyna Hull, 1941, Salpingogaster ................

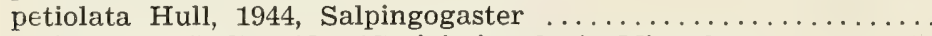

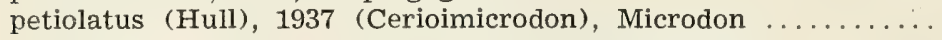
phaeopterus (Schiner), 1868 (Baccha), Ocyptamus ..........

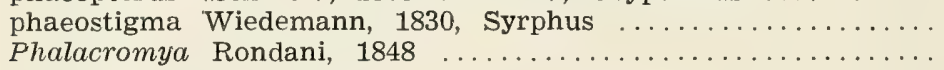

Phalacromyia, auctorum

philippi (Shannon), 1925 (Cerioides), Sphiximorpha .........

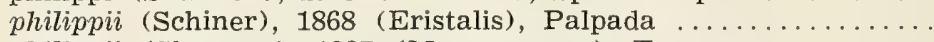
philippii (Shannon), 1927 (Mesogramma), Toxomerus ........

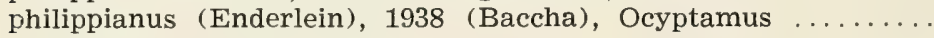

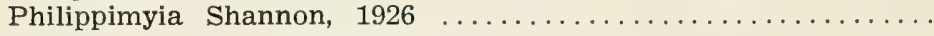

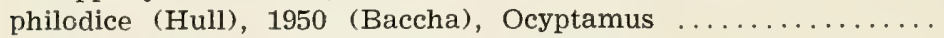

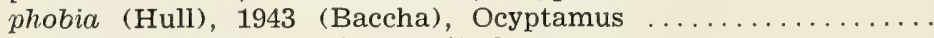

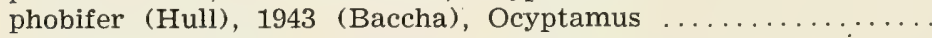
Pia Philippi, 1865

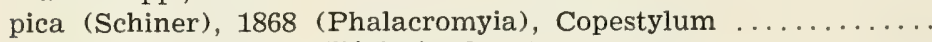

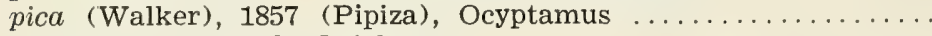

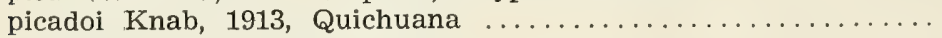

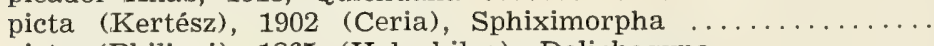

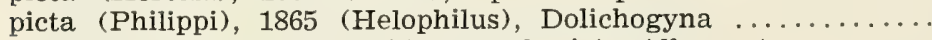
picticauda (Bigot), 1884 (Sphaerophoria), Allograpta ........

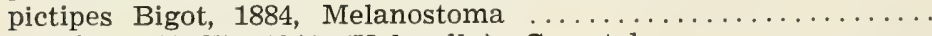

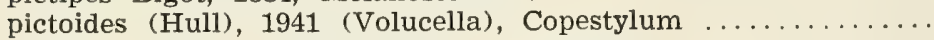

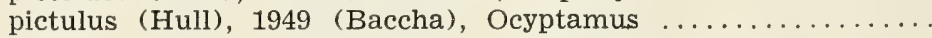
pictum (Schiner), 1868 (Phalacromyia), Copestylum ......... pictum (Wiedemann), 1830 (Volucella), Copestylum ..........

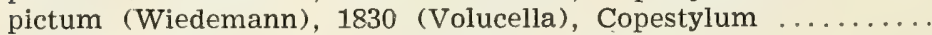

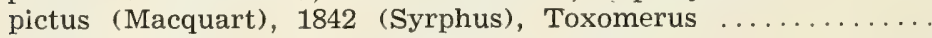

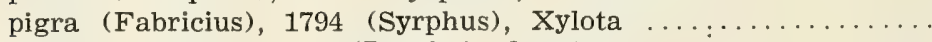

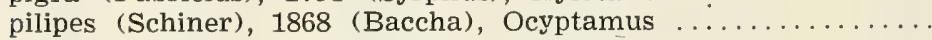

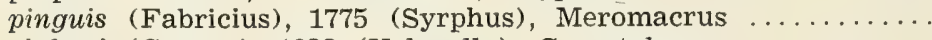

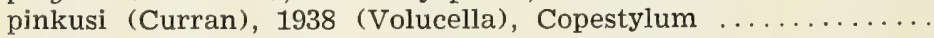

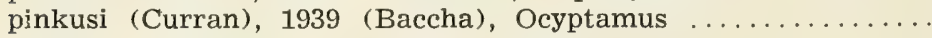

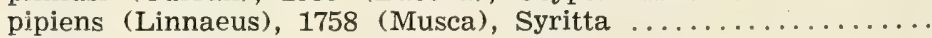

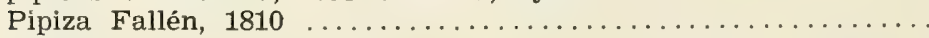
Pipizini

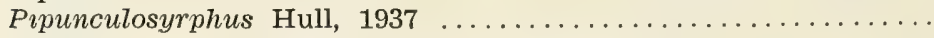

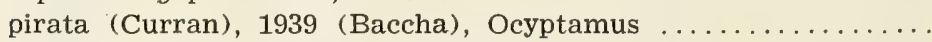

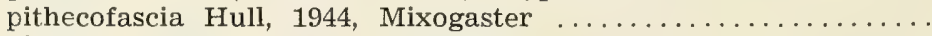

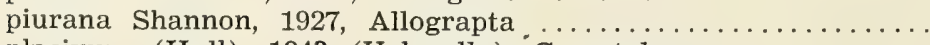

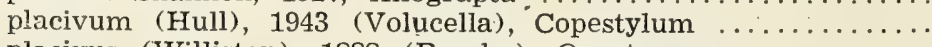

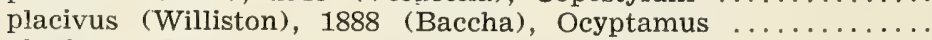

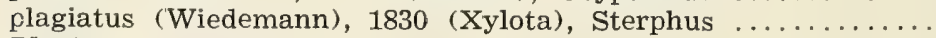

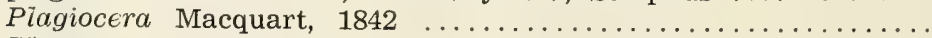

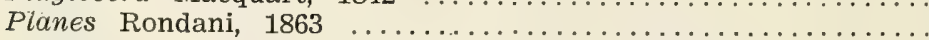

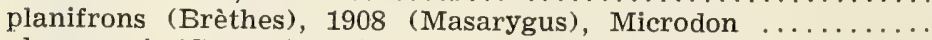

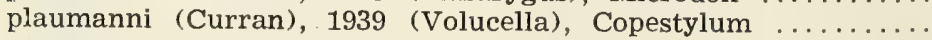

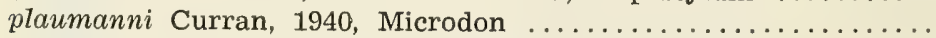

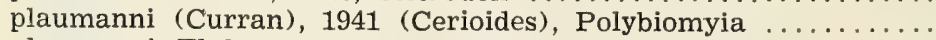
plaumanni Fluke, 1937, Xanthandrus 
plaumanni (Frey), 1946 (Mocrosphaerophoria), Allograpta ...

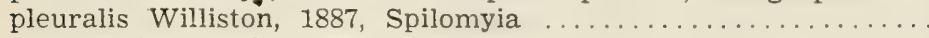

plorans (Rondani), 1848 (Volucella), Copestylum ...........

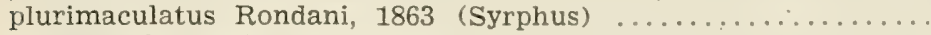

pluto Hull, 1942, Meromacrus

46.121

46.82

46.39

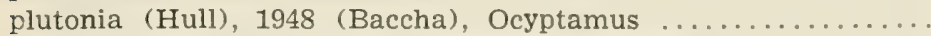

podagra (Macquart), 1842 (Eristalis), Palpada

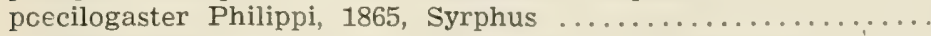

poєcilogastrus (Loew), 1865 (Mesogramma), Toxomerus .......

pcgonosa Fluke, 1937, Quichuana

46.113

46.25

46.105

46.8

46.53

46.99

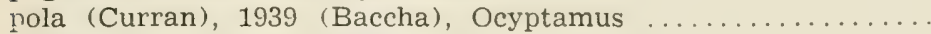

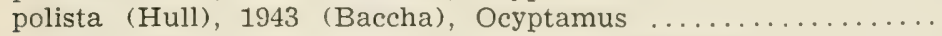

polistes (Curran), 1941 (Cerioides), Sphiximorpha ..........

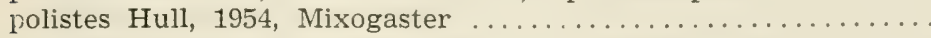

polistiformes (Hull), 1944 (Cerioides), Sphiximorpha .........

Polistoceria Hull, 1949

46.25

46.25

46.94

46.58

46.94

46.93

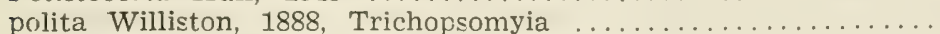

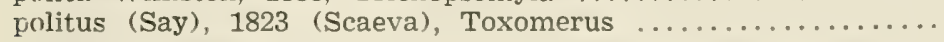

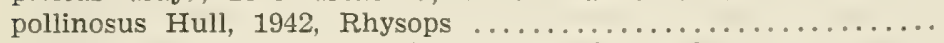

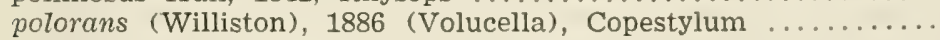
Polybiomyia Shannon, 1925

Polydonta Macquart, 1850

Polydontomyia Williston, 1896

polygrammus (Loew), 1872 (Helophilus) A.................. polygraphicus (Hull), 1940 (Mesogramma), Toxomerus ........

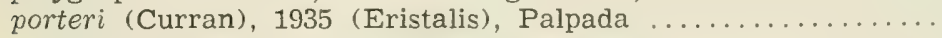
porticola (Thomson), 1869 (Syrphus), Toxomerus ...........

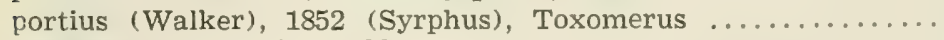

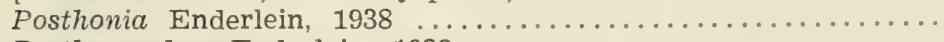

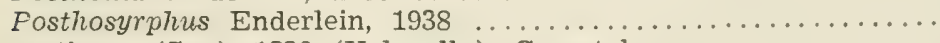

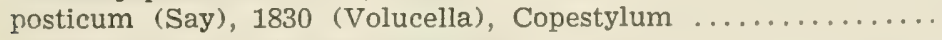

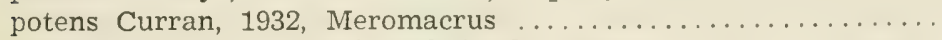

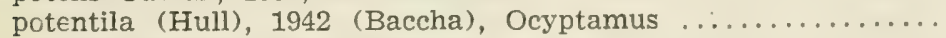

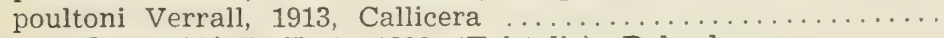
praeclarus (Giglio-Tos), 1892 (Eristalis), Palpada ...........

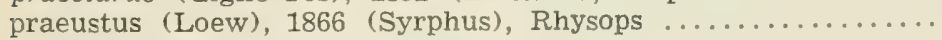
prasinus (Schiner), 1868 (Phalacromyia), Copestylum ......... pratorum (Fabricius), 1775 (Syrphus), Meromacrus ...........

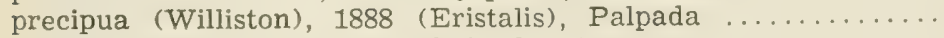

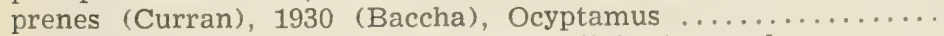

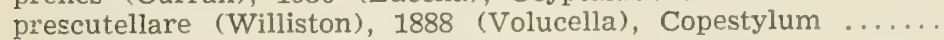
pretiosa Loew, 1861, Xylota

prima (Curran), 1941 (Aristosyrphus), Argentinomyia ........

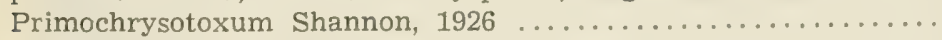

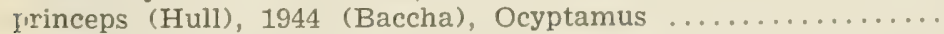

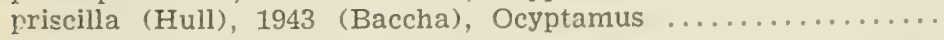

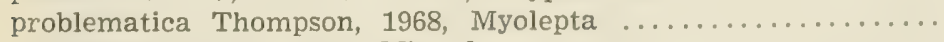

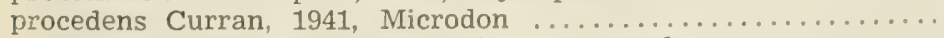
procteri (Curran), 1939 (Volucella), Copestylum ............

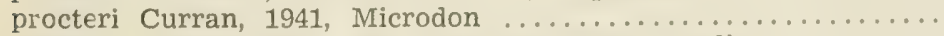

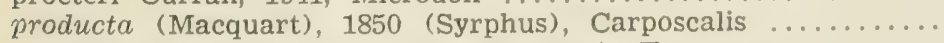
productus (Curran), 1930 (Mesogramma), Toxomerus ........ Fromilesia Lynch Arribálzaga, $1892 \ldots \ldots \ldots \ldots \ldots \ldots \ldots$ Protoceratophya Hull, 1949 Protolepidostala Hull, 1949

46.68

46.53

46.44

46.82

46.95

46.96

46.96

46.96

46.54

46.104

46.54

46.54

46.9

46.9

46.82

46.113

46.25

46.88

46.108

46.44

46.82

46.113

46. 108

46.25

46.82

46.114

46.57

46.40

46.26

46.26

46.90

46.62

46.82

46.62

46.41

46.54

46.111

46.57

46.90 


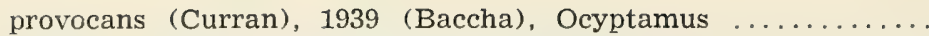

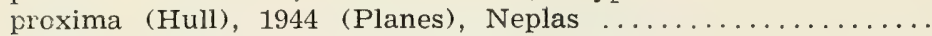

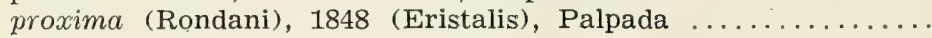

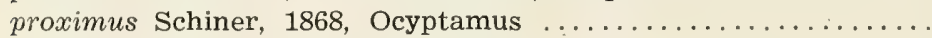

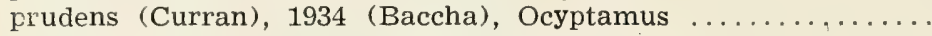

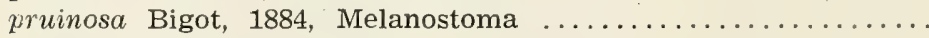

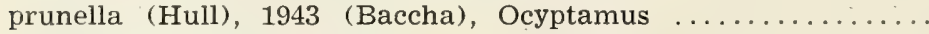

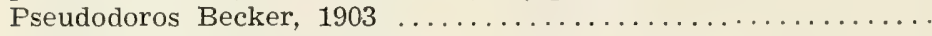

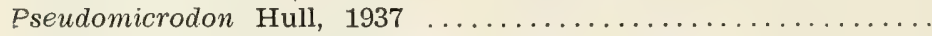
pseudoribesii Vimmer \& Soukup, 1938, Syrphus ...........

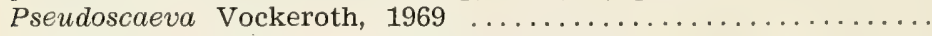
pseudotachina Hull, 1930, Tachinosyrphus $\ldots \ldots \ldots \ldots \ldots \ldots$

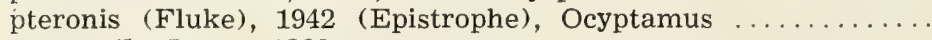
Pteroptila Loew, 1866 pubescens (Loew), 1861 (Volucella), Copestylum ............ pubescens (Shannon \& Aubertin), 1933 (Chilosia), Chromocheilosia

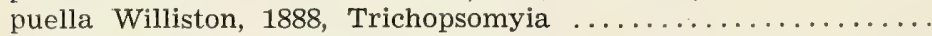

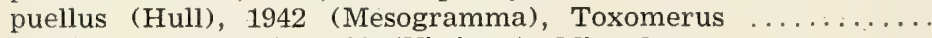

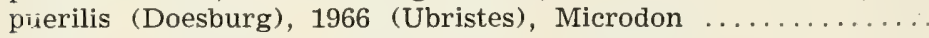

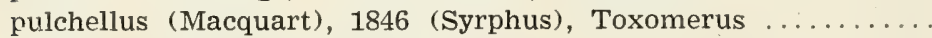

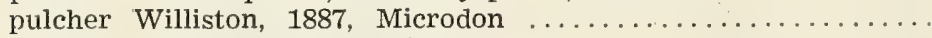

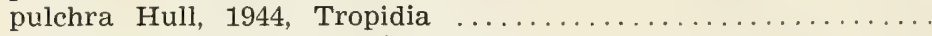

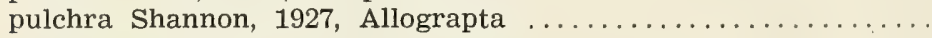
pulchra (Williston), 1888 (Lepidostola), Lepidomyia ..........

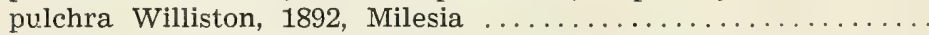
pulchrapuella (Hull), 1943 (Volucella), Copestylum ......... pulchripes (Bigot), 1875 (Volucella), Copestylum ........... pulchrum (Williston), 1891 (Phalacromyia), Copestylum .....

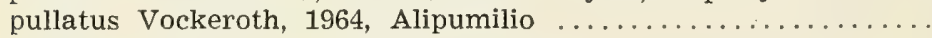

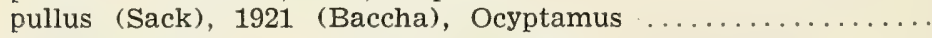

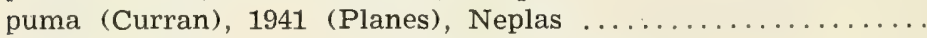

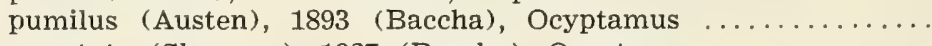

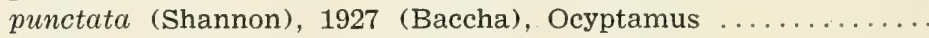

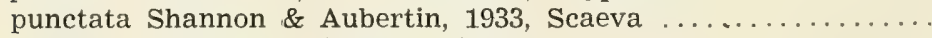

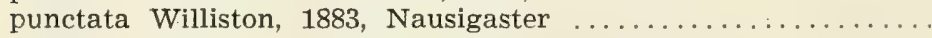

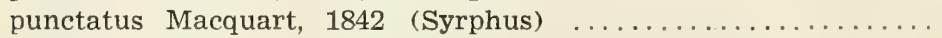

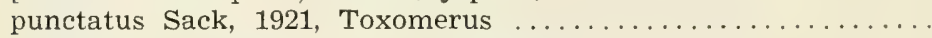
punctiferum (Bigot), 1875 (Volucella), Copestylum .........

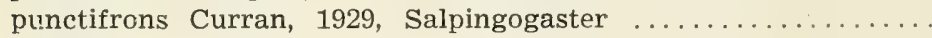

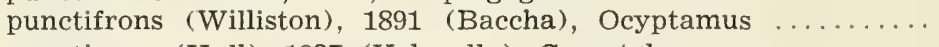
punctigena (Hull), 1937 (Volucella), Copestylum punctulata (Wulp), 1888 (Melanostoma), Carposcalis purpurascens (Loew), 1869 (Temnocera), Copestylum ............ purpureum (Walker), 1849 (Volucella), Copestylum ......... purpuriferum (Bigot), 1875 (Volucella), Copestylum ......... purus (Curran), 1930 (Mesogramma), Toxomerus .............

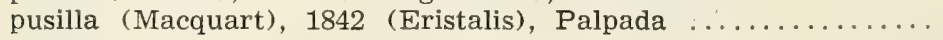
pusillum (Macquart), 1842 (Volucella), Copestylum ..........

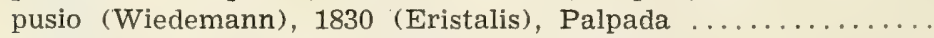

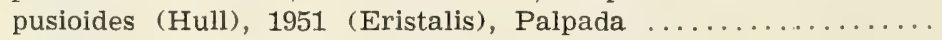

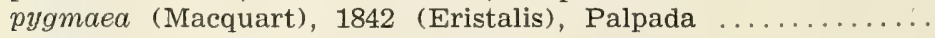

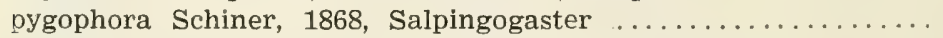
pygolampa (Wiedemann), 1830 (Eristalis), Palpada .......... pyrastri Linnaeus, of Lynch Arribálzaga, 1892, Scaeva

46.26

46.115

46.105

46.27

46.26

46.42

46.26

46.10

46.59

46.8

46.11

46.70

46.26

46.111

46.82

46.90

46.68

46.54

46.62

46.54

46.66

46.120

46.37

46.89

46.120

46.82

46.82

46.82

46.92

46.26

46.115

46.26

46.12

46.10

46.93

46.39

46.54

46.82

46.32

46.26

46.82

46.41

46.82

46.82

46.82

46.54

46.108

46.83

46.108

46.108

46.106

46.32

46.108

46.9 
Wyrrhina Bigot, 1884, Sphaerophoria ............... 46.38

pyrrhocera (Kertész), 1903 (Ceria), Sphiximorpha .......... 46.94

pJxia (Hull), 1943 (Baccha), Ocyptamus ................. 46.26

quadraticornis (Macquart), 1842 (Eristalis), Palpada ....... 46.107

quadratum (Williston), 1891 (Volucella), Copestylum ........ 46.83

quadricincta Enderlein, 1938, Allograpta ................. 46.37

quadrigemina (Thomson), 1869 (Syrphus), Allograpta .......... 46.35

quadrilineatus (Enderlein), 1938 (Hybobathus), Toxomerus ... 46.54

quadrimaculata Enderlein, 1938, Allograpta ............ 46.36

quadrimaculatus Hull, 1944, Rhysops .................. 46.44

quadrinctatus (Bigot), 1884 (Melanostoma), Xanthandrus .... 46.45

quadripunctatum (Doesburg), 1963 (Volucella), Copestylum ... 46.81

quadristriata (Shannon \& Aubestin), 1933 (Chrysogaster), Or-

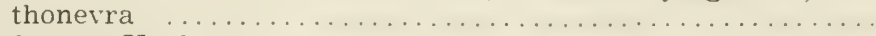

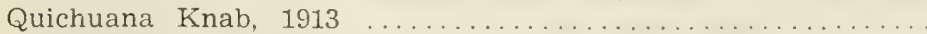

Quihuana Hull, 1944

quinquecinctus (Bigot), 1888 (Mesograpta), Toxomerus .......

quinquemaculatus (Bigot), 1888 (Mesograpta), Toxomerus .....

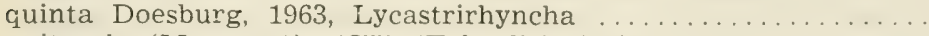

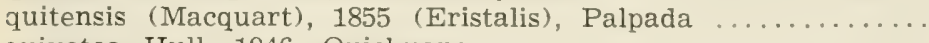

quixotea Hull, 1946, Quichuana

46.91

46.98

46.98

46.54

46.54

46.102

46.108

46.99

radiatus Bigot, 1857 (Syrphus) . . . . . . . . . . . . . . 46.39

rafaelanum (Townsend), 1897 (Volucella), Copestylum ...... 16.83

rarior Shannon, 1925, Mixogaster ................... 46.59

rarissima Shannon, 1925, Mixogaster ................. 46.59

rectilinea Hull, 1942, Habromyia ..................... 46.101

rectum (Wulp), 1882 (Temnocera), Copestylum ........... 46.83

røedi Shannon, 1927, Syrphus ..................... 46.8

relicta Curran, 1941, Salpingogaster ............... 46.32

remigis (Fluke), 1942 (Epistrophe), Allograpta ........... 46.37

remotus Knab, 1917, Microdon .................... 46.66

rcmus Curran, 1941, Microdon ..................... 46.66

reynoldsi Shannon \& Aubertin, 1933, Dolichogyna .......... 46.98

reynoldsi (Shannon \& Aubertin), 1933 (Melanostoma), Carposcalis 46.41

rex (Fluke), 1945 (Melanostoma), Rhysops .............. 46.44

rinea (Hull), 1949 (Mesogramma), Toxomerus .............. 46.54

rhea (Hull), 1950 (Volucella), Copestylum ............. 46.83

rheochryssus Hull, 1944, Microdon ................. 46.66

rhina Thompson, Allograpta ....................... 46.37

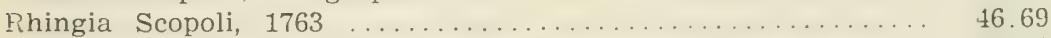

Rhinoprosopa Hull, $1942 \ldots \ldots \ldots \ldots \ldots \ldots \ldots \ldots \ldots \ldots \ldots . \ldots \ldots . \ldots \ldots$

rhodope (Hull), 1951 (Mesogramma), Toxomerus ........... 46.54

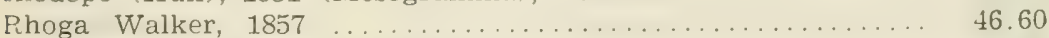

rhombicus, auctorum, Toxomerus …............. 46.54

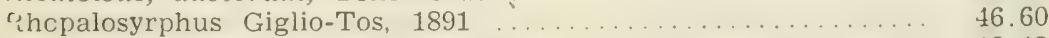

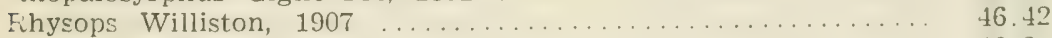

ribesii, auctorus, Syrphus ............................. 46.

rea (Curran), 1939 (Volucella), Copestylum ............. 46.75

ricus (Curran), 1939 (Baccha), Ocyptamus .............. 46.26

rieseli Shannon, 1927, Quichuana ..................... 46.99

rileyi (Williston), 1887 (Doliosyrphus), Palpada ........... 46.109

robinsoniana Enderlein, 1940 , Allograpta .............. 46.37

roburoris (Fluke), 1942 (Epistrophe), Allograpta .......... 46.37 


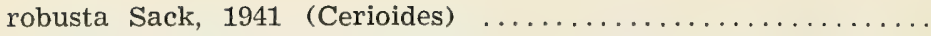
robustum (Sack), 1941 (Temnocera), Copestylum ........... roederii (Williston), 1888 (Ceria), Sphiximorpha ...........

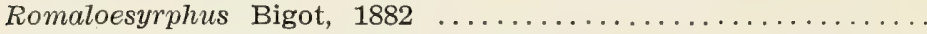
rombicus (Giglio_Tos) 1892 (Mesogramma), Toxomerus .....

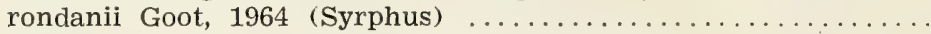

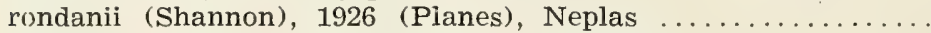
roraima (Curran), 1939 (Volucella), Copestylum .............

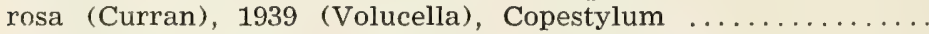
rospigliosii (Brèthes), 1920 (Volucella), Copestylum .......... rostrata (Bigot), 1884 (Sphaerophoria), Allograpta ............ costrata (Macquart), 1846 (Helophilus), Dolichogyna ........

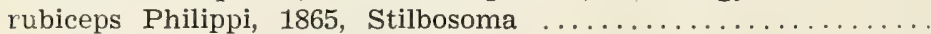

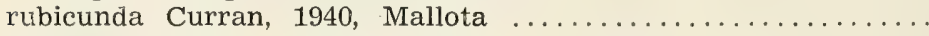
rubida (Williston), 1891 (Baccha), Leucopodella ............

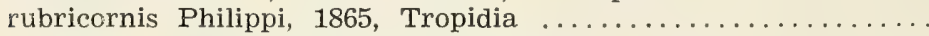
rubricosus (Wiedemann), 1830 (Syrphus), Ocyptamus ........ rubriventris Lynch Arribálzaga, 1891, Microdon ............ rubrobrunnea (Hull), 1944 (Cerioides), Sphiximorpha ........

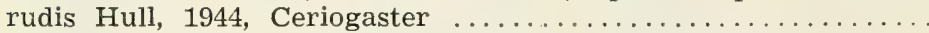
rufibasis (Bigot), 1883 (Sphiximorpha), Polybiomyia ........ ruficauda (Shannon), 1927 (Valdivia), Valdiviomyia .......... ruficaudatus Bigot, 1884 (Paragus), Toxomerus ............

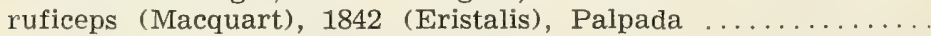

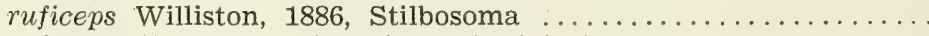

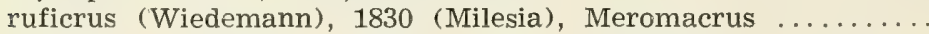

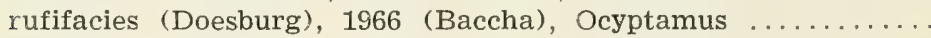
rufipedes Thompson, Palpada

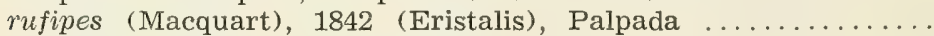

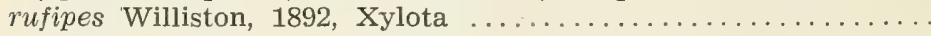

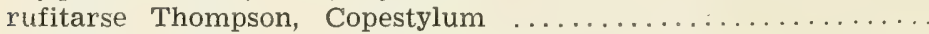

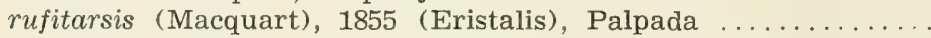

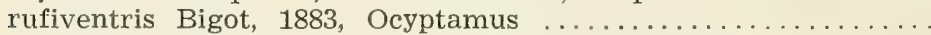

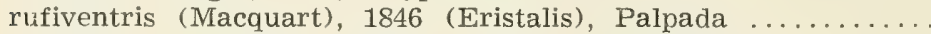

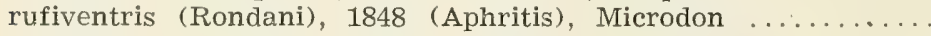
rufocinctus (Curran), 1927 (Mesogramma), Toxomerus ......

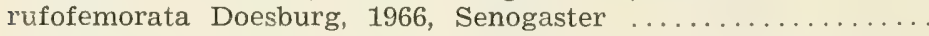
rufoscutata Bigot, 1880, Eristalis

rufoscutellare (Philippi), 1865 (Phalacromyia), Copestylum ... rufoscutellata (Sack), 1921 (Eristalis), Palpada .............

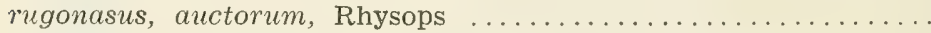

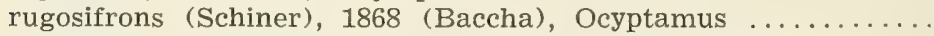
rugosonasus (Williston), 1891 (Melanostoma), Rhysops ......

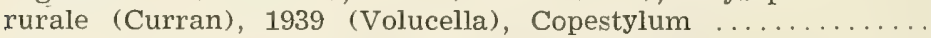

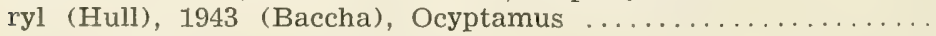

sackeni Williston, 1882, Mallota 
saltana Enderlein, 1938, Carposcalis

46.41

salti Curran, 1929, Mallota

46.100

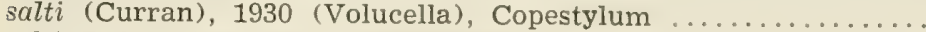

46.84

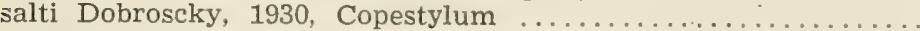

46.83

saphiridiceps (Bigot), 1884 (Mesograpta?), Toxomerus ........ saphirinum (Bigot), 1883 (Volucella), Copestylum ........... sapphirina Hull, 1951, Neplas ....................... sapphirina (Shannon \& Aubertin), 1933 (Volucella), Copestylum

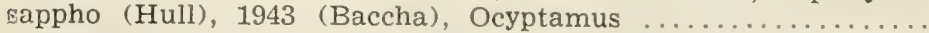

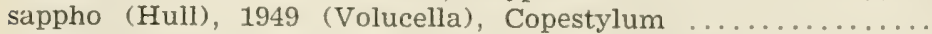

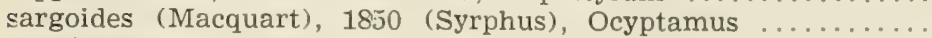
Sarolepta Hull, 1941

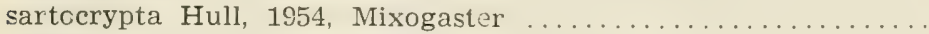

46.54

46.83

46.115

46.83

46.26

46.83

46.27

46.89

46.59

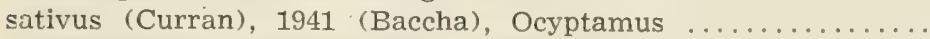

46.27

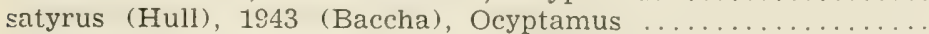

saussurii (Giglio-Tos), 1892 (Syrphus), Allograpta ..........

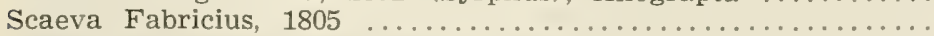

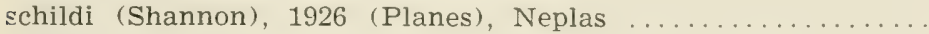

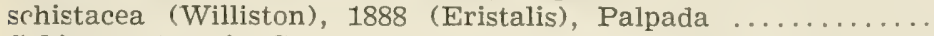

Schizoceratomyia Carrera, Lopes \& Lane, 1947 ...........

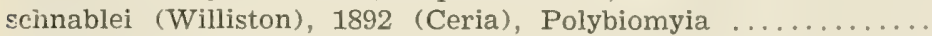

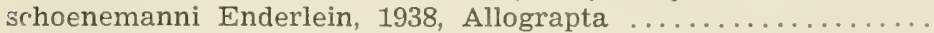

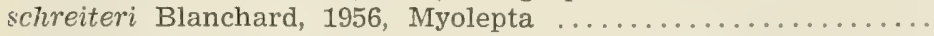

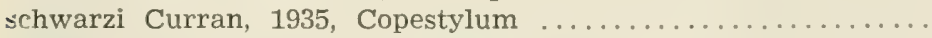

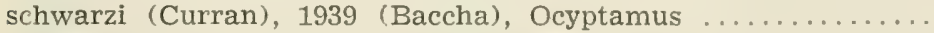

schwarzi Shannon, 1925, Polybiomyia

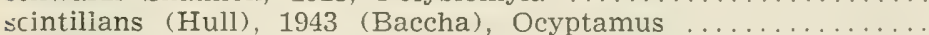

scintillans (Hull), 1946 (Lepidostola), Myolepta .............

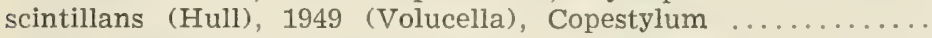

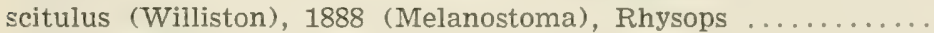

scitus (Walker), 1857 (Helophilus), Meromacrus ...........

scolopus Shannon, 1927, Microdon

46.27

46.37

46.9

46.115

46.109

46.60

46.95

46.37

46.89

46.83

46.27

46.95

46.27

46.90

46.83

46.44

46.113

46.62

scopanthus (Hull), 1944 (Eristalis), Palpada ..............

46.109

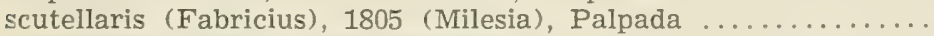

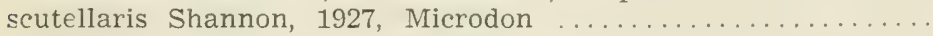

46.109

46.62

scutellaris (Walker), 1837 (Paragus), Pseudodoros ...........

?scutellata (Bigot), 1883 (Doliosyrphus), Palpada ..........

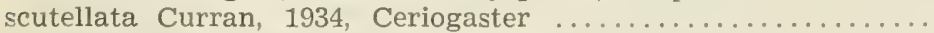
scutellata Hull, 1924, Milesia

46.11

46.109

46.116

46.120

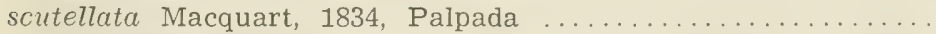

46.109

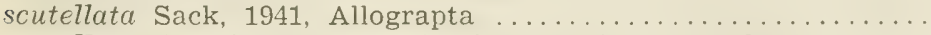

46.34

scutellata (Williston), 1886 (?Mixogaster), Pseudodoros ......

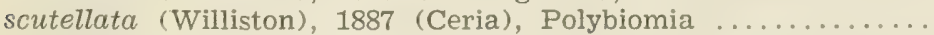

scutellatum (Macquart), 1842 (Volucella), Copestylum ........ scutellatum (Williston), 1888 (Apophysophora), Copestylum ...

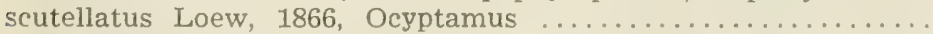
scutigera (Fluke), 1945 (Melanostoma), Carposcalis .......... seabrai (Papavero), 1962 (Pseudomicrodon), Microdon ........ selectum (Curran), 1939 (Volucella), Copestylum ........... sclene (Hull), 1949 (Baccha), Ocyptamus

selva (Hull), 1943 (Mesogramma), Toxomerus ............. semicirculus (Walker), 1852 (Eristalis), Palpada ........... senicula (Loew), 1866 (Eristalis), Palpada Senoceria Hull, 1930

46.11

46.95

46.83

46.85

46.27

46.41

46.66

46.83

46.27

46.54

46.109

46.103

46.117

Senogaster Macquart, 1834

46.119 


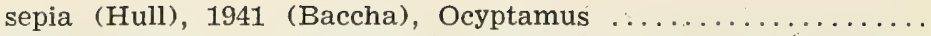

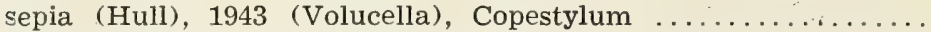

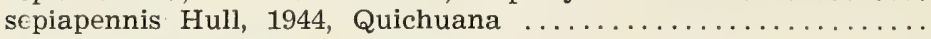

sepulchrasilvus (Hull), 1937 (Papiliomyia), Microdon .........

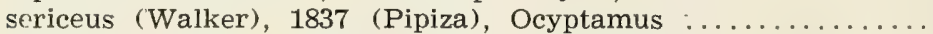

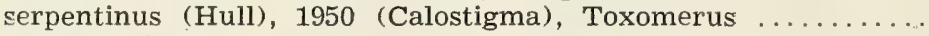

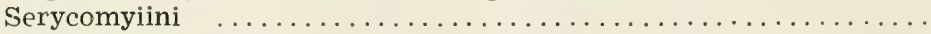

setigera (Osten Sacken), 1877 (Temnocera), Copestylum .....

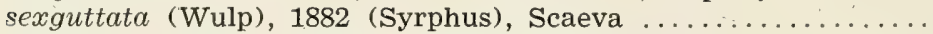

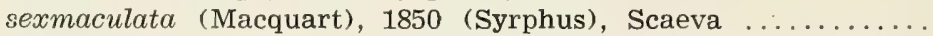
sexmaculatum (Palisot de Beauvois), 1819 (Syrphus), Copestylum sexpunctatum (Loew), 1861 (Volucella), Copestylum ......... sexpunctatum (Loew), 1861 (Volucella), Copestylum ......... shannoni Carrera, Lopes \& Lane, 1947, Nausigaster .......... shannoni (Curran), 1925 (Chrysogaster), Orthonevra ........

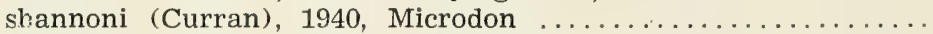

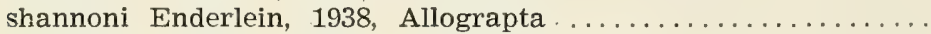
shannoni (Lane \& Carrera), 1943 (Cerioides), Sphiximorpha ...

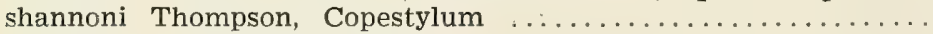

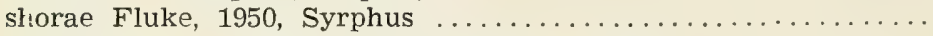
shropshirei (Curran), 1930 (Baccha), Ocyptamus ............

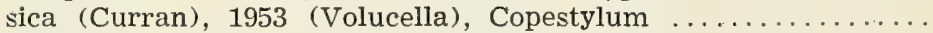

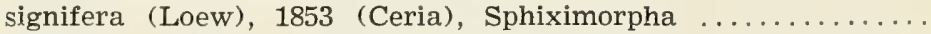

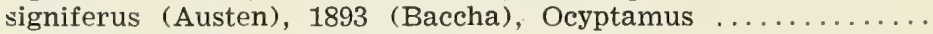

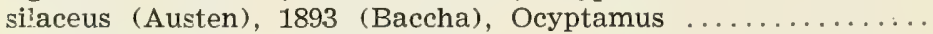
simile Giglio-Tos; 1892, Copestylum ...................

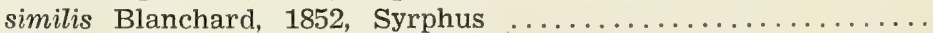

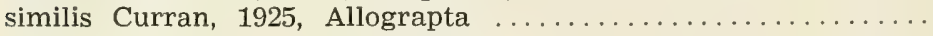

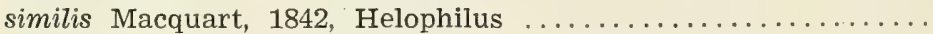
similis (Williston), 1888 (Lepidostola), Lepidomyia ........... simillima (Vimmer \& Soukup), 1938 (Syrphus), Scaeva .......

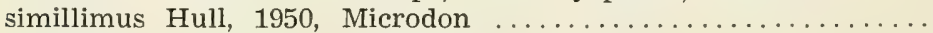

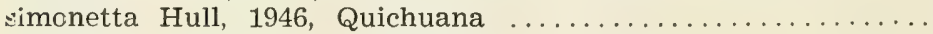

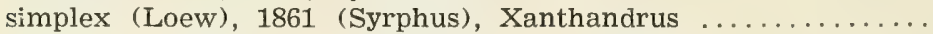

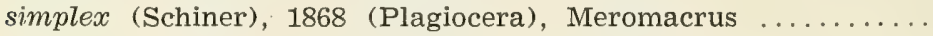

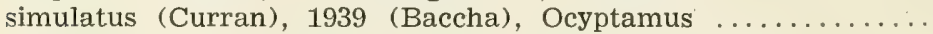
sinuatinervis (Macquart), 1850 (Syrphus), Toxomerus ....... skinneri (Johnson), 1903 (Chilosia), Chamaesyrphus .........

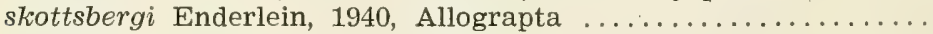
slossonae (Curran), 1930 (Mesogramma), Toxomerus .........

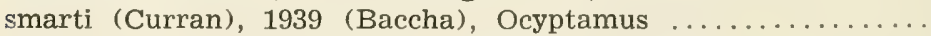

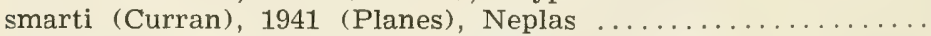
smithae (Thompson), 1965 (Lepidosis), Copestylum ..........

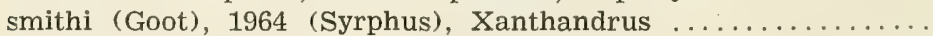

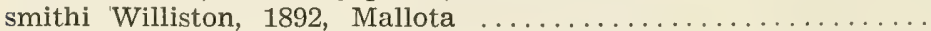
sodomis (Townsend), 1895 (Volucella), Copestylum ..........

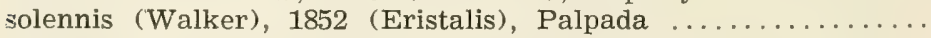

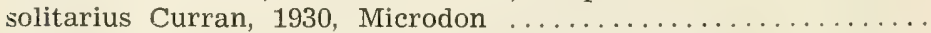

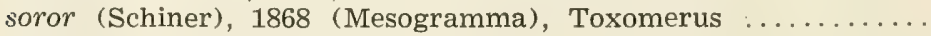

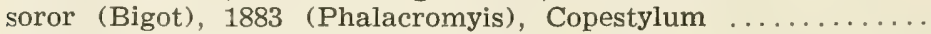

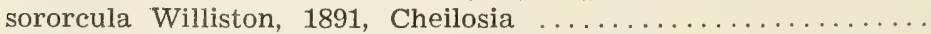

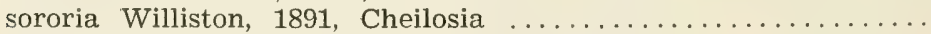
soukupii (Vimmer \& Soukup), 1938 (Volucella) ............

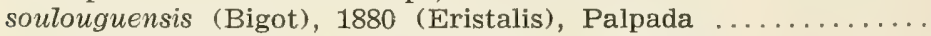
spatulatus (Giglio-Tos), 1892 (Baccha), Ocyptamus 
spectabilis (Hull), 1925 (Eristalis), Palpada

46.109

spectrale (Hull), 1942 (Volucella), Copestylum .............

Snhaerophoria Lepeletier \& Serville, $1928 \ldots \ldots \ldots \ldots \ldots \ldots \ldots$

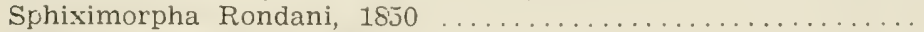

Sphyximorpha, auctorum

Spilcmyia Meigen, 1803

spinifemoratus (Hull), 1930 (Senoceria), Sterphus ............

spinigerum (Wiedemann), 1830 (Volucella), Copestylum ..... spinithorax (Lynch Arribálzaga), 1892 (Temnocera), Copestylum spinosa (Shannon), 1925 (Zonemyia), Ceriogaster

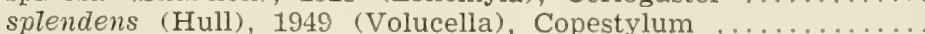

splendens (Thomson), 1869 (Syrphus), Allograpta ................

splendens (Townsend), 1897 (Volucella), Copestylum .........

splendens Wiedemann, 1830, Microdon

squamigerum (Curran, 1925 (Volucella), Copestylum .........

steatogaster (Hull), 1941 (Mesogramma), Toxomerus ........

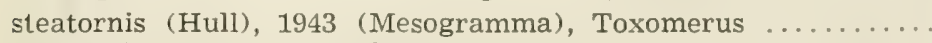

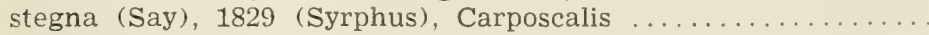

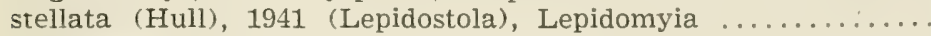

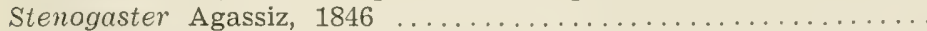

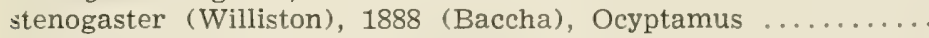

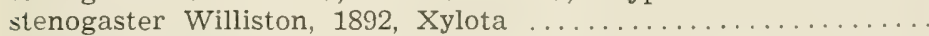

sternale (Curran), 1930 (Volucella), Copestylum ............

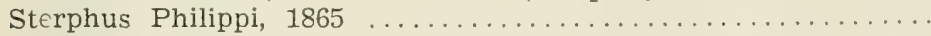

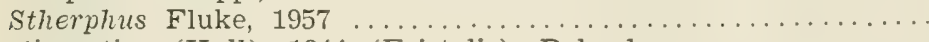

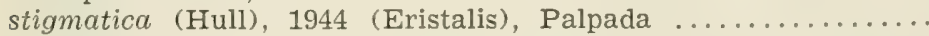

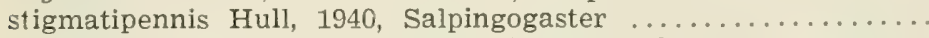

stigmatum (Hull), 1949 (Volucella), Copestylum ............

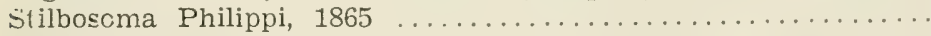

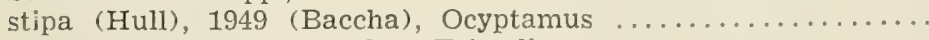

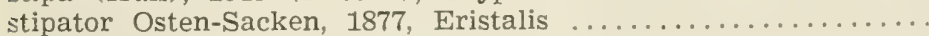

Stipomorpha Hull, 1945

46.81

46.38

46.93

46.93

46.121

46.117

46.84

46.84

46.116

46.80

46.37

46.84

46.66

46.84

46.55

46.55

46.41

46.89

46.119

46.27

46.114

46.84

46.116

46.116

46.107

46.32

46.84

46.121

46.28

46.102

46.61

46.28

46.67

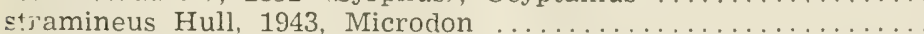

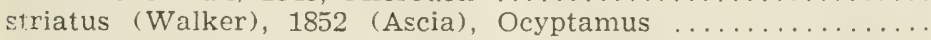

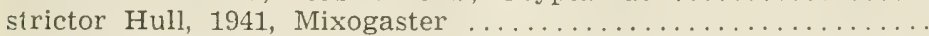

strigifacies (Enderlein), 1938 (Fazia), Allograpta ..........

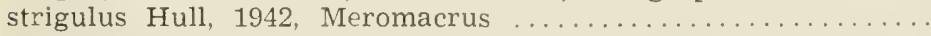

Styxia Hull, 1943

subannulatus (Loew), 1865 (Mesograpta), Toxomerus ........

subchalybeus (Walker), 1857 (Syrphus), Ocyptamus .............

subcoeruleum (Rondani), 1863 (Phalacromyia), Copestylum ...

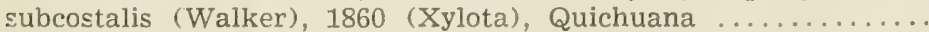

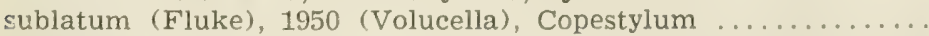
submetallicum (Rondani), 1848 (Phalacromya), Copestylum

46.28

46.59

46.37

46.113

46.11

46.55

46.28

46.81

46.99

46.84

46.85

46.85

sulphuripes (Thomson), 1869 (Syrphus), Sphaerophoria . . . . . . 4.38
sultzi (Curran), 1939 (Volucella), Copestylum .............. 46.85

sumichrasti (Giglio-Tos), 1893 (Eristalis), Palpada ......... 46.110

sumischarasti (Giglio-Tos), 1892 (Eristalis), Palpada ......... 46.110

summus (Fluke), 1936 (Baccha), Ocyptamus ............... 46.28

superba (Williston), 1887 (Ceria), Sphiximorpha ............ 46.94

superbus Wiedemann, 1830, Microdon .................. 46.67

surinamensis (Degeer), 1776 (Musca), Palpada .............. 46.110

surinamensis (Macquart), 1842 (Eristalis), Palpada .......... 46.105 


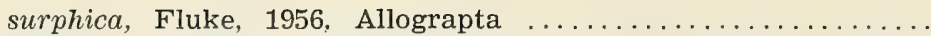

susio (Hull), 1941 (Baccha), Ocyptamus ............... 46.28

sycorax (Hull), 1947 (Rhinoprosopa), Allograpta .......... 46.37

sylphus (Hull), 1943 (Mesogramma), Toxomerus ........... 46.52

sylvaticus (Hull), 1943 (Mesogramma), Toxomerus ......... 46.55

sylvicola Knab, 1913, Quichuana ...................... 46.99

Syritta Lepeletier \& Serville, 1828 . . . . . . . . . . . . . . . 46.119

syrphica (Giglio-Tos), 1892 (Sphaerophoria), Allograpta ...... 46.37

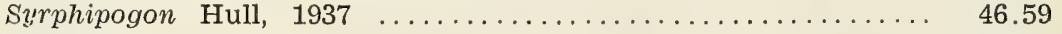

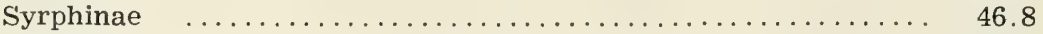

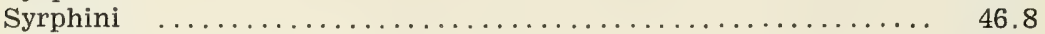

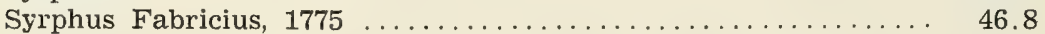

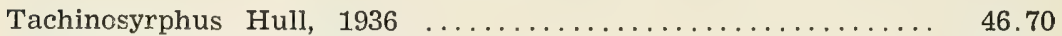

taenia (Wiedemann), 1830 (Eristalis), Palpada ............. 46.109

taenius (Curran), 1930 (Mesogramma), Toxomerus ......... 46.55

Talahua Fluke, 1945 ......................... 46.45

tamaulipanum (Townsend), 1898 (Volucella), Copestylum .... 46.85

tapeta Fluke, 1939, Criorhina .................... 46.119

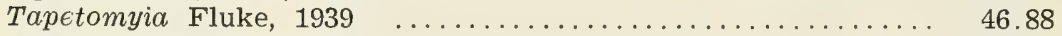

tursalis (Walker), 1837 (Syrphus), Ocyptamus ............ 46.28

tatei (Curran), 1930 (Eristalis), Palpada .................. 46.109

tatei (Curran), 1930 (Volucella), Copestylum ............. 46.85

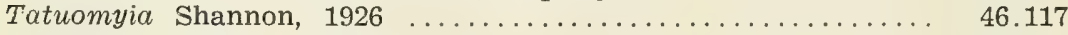

tau (Bigot), 1883 (Volucella), Copestylum ............ 46.85

tectiforma Fluke, 1942, Allograpta .................... 46.38

teffera (Curran), 1939 (Volucella), Copestylum ............... 46.85

telescopicus (Curran), 1930 (Baccha), Ocyptamus ........... 46.28

teligera Fluke, 1942, Allograpta ................... 46.38

tenax (Linnaeus), 1758 (Musca), Eristalis .............. 46.101

tenebrica (Walker), 1849 (Merodon), Palpada ............. 46.106

tenuicaudus Curran, 1925, Microdon .................. 46.62

tenuifrons Curran, 1930, Eristalis ....................... 46.102

tenuis (Walker), 1852 (Baccha), Ocyptamus .............. 46.28

testaceicornis (Macquart), 1850 (Eristalis), Palpada ......... 46.109

testaceicornis Macquart, 1850, Syrphus ............... 46.8

tsetaceipes Lynch Arribálzaga, 1891, Argentinomyia .......... 46.57

testaceiscutellata (Macquart), 1850 (Eristalis), Palpada ....... 46.107

testaceum (Rondani), 1848 (Volucella), Copestylum ......... 46.81

testaceum (Wulp), 1891 (Volucella), Copestylum ............ 46.87

Thatamopales Hull, 1949 ....................... 46.111

thalia (Hull), 1942 (Eristalis), Palpada ................. 46.110

thecla (Hull), 1943 (Baccha), Ocyptamus ................. 46.28

thecla Hull, 1954, Mixogaster ........................ 46.59

Therantha Hull, 1943 ............................ 46.11

thiemei (Enderlein), 1938 (Braziliana), Rhysops .......... 46.44

thoracica (Jaennicke), 1867 (Eristalis), Palpada ............. 46.108

trara (Curran), 1941 (Baccha), Ocyptamus ................ 46.19

tiarella (Hull), 1944 (Baccha), Ocyptamus .............. 46.28

tibiale (Macquart), 1846 (Volucella), Copestylum ........... 46.85

tibiale (Shannon), 1929 (Volucella), Copestylum .......... 46.83

tibialis (Fallén), 1817 (Pipiza), Paragus ................ 46.40

tibicen (Wiedemann), 1830 (Syrphus), Toxomerus .......... 46.55

tigrinus Curran, 1940, Microdon ................... 46.67 
timberlakei (Curran), 1926 (Vollucella), Copestylum

tinctus (Fluke), 1950 (Crepidomyia), Sterphus

titan (Hull), 1947 (Baccha), Ocyptamus

46.13

titania (Hull), 1943 (Baccha), Ocyptamus ............. 46.28

titillans Hull, 1944, Lycastrirhyncha ................ 46.102

toltec (Townsend), 1895 (Volucella), Copestylum .......... 46.78

tolteca (Townsend), 1895 (Volucella), Copestylum .......... 46.78

townsendi (Goot), 1964 (Volucella), Copestylum ........... 46.84

townsendi (Snow), 1895 (Ceria), Polybiomyia ............ 46.95

Toxomerini .......................... 46.47

Toxomerus Macquart, 1855 ...................... 46.47

trabis (Fluke), 1942 (Epistrophe), Ocyptamus ............ 46.28

transatlanticum (Rondani), 1863 (Volucella), Copestylum ..... 46.75

transatlanticus (Schiner), 1868 (Baccha), Ocyptamus ....... 46.28

transversa (Walker), 1857 (Syritta), Ceriogaster ........... 46.116

transversa (Hine), 1913 (Myiolepta), Ceriogaster ........... 46.116

transversa (Hull), 1943 (Sphaerophoria), Allograpta ........ 46.38

travassosi (Lane \& Carrera), 1943 (Cerioides), Polybiomyia ... 46.95

triangularis Curran, 1940, Microdon ................ 46.62

triangularis (Giglio-Tos), 1892 (Eristalis), Palpada .......... 46.110

triangulatus (Hull), 1942 (Mesograma), Toxomerus ......... 46.55

tribinicincta Enderlein, 1938, Allograpta ................ 46.38

trichopoda (Kertész), 1903 (Ceria), Sphiximorpha .......... 46.94

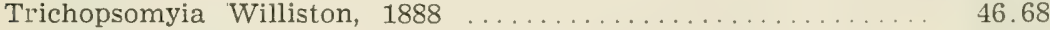

tricinctum (Bigot), 1875 (Volucella), Copestylum .......... 46.85

tricinctus (Bigot), 1883 (Baccha), Ocyptamus ............ 46.28

tricinctus (Wulp), 1888 (Baccha), Ocyptamus ............. 46.30

tricolor (Loew), 1861 (Ceria), Monoceromyia ............. 46.94

tricolor (Jaennicke), 1867 (Eristalis), Palpada ............ 46.108

tricrepis (Shannon), 1926 (Crepidomyia), Sterphus ........ 46.117

tridentatus (Rondani), 1868 (Syrphus), Toxomerus .......... 46.55

trifasciata Enderlein, 1938, Allograpta .................. 46.38

trifasciata (Macquart), 1842 (Eristalis), Palpada ............ 46.103

trifasciata (Say), 1830 (Eristalis), Palpada ............... 46.110

trifascium (Walker), 1857 (Temnocera), Copestylum ........ 46.85

trigona (Williston), 1891 (Eristalis), Palpada ............ 46.110

trigoniformis Shannon, 1927, Microdon ............... 46.62

trigonum (Giglio_Tos), 1892 (Volucella), Copestylum ........ 46.85

trigonus (Hull), 1937 (Hypselosyrphus), Microdon .......... 46.62

trigonus (Wiedemann), 1830 (Syrphus), Ocyptamus ......... 46.19

trilimbata (Bigot), 1888 (Sphaerophoria), Allograpta ......... 46.38

trilımbata (Giglio-Tos), 1892 (Eristalis), Palpada ........... 46.110

trilinea Hull, 1943, Microdon ........................ 46.67

trilineata (Hull), 1941 (Lepidostola), Lepidomyia ........... 46.89

trilineatus (Enderlein), 1938 (Mesogramma), Toxomerus ..... 46.55

trilobatus (Bigot), 1884 (Mesograpta?), Toxomerus ......... 46.55

trilobus (Hull), 1944 (Baccha), Ocyptamus .............4 46.28

trinidadesis (Curran), 1939 (Baccha), Ocyptamus ........... 16.28

Triodonta Williston, $1885 \ldots \ldots \ldots \ldots \ldots \ldots \ldots \ldots \ldots \ldots \ldots . \ldots \ldots . \ldots \ldots$

tripunctatum (Hull), 1949 (Volucella), Copestylum ......... 46.85

triradiatus (Hull), 1942 (Mesogramma), Toxomerus ........ 46.55

triste (Bigot), 1875 (Volucella), Copestylum ............ 46.85

tristis (Hull), 1930 (Baccha), Ocyptamus ................ 46.29

tristis (Philippi), 1865 (Penium), Pipiza ................ 46.68

trituberculatum Thompson, Copestylum ............. 46.85 


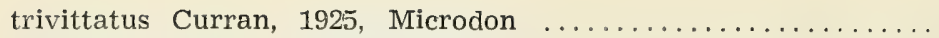

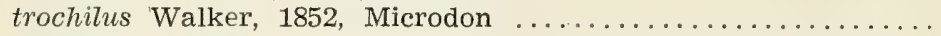
tropicus (Curran), 1937 (Melanostoma), Rhysops ..............

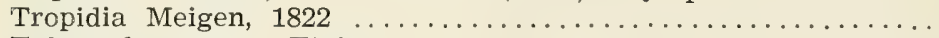

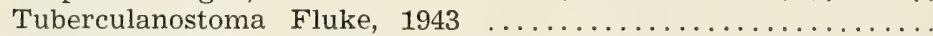
tuberculata Carrera, Lopes \& Lane, 1947, Nausigaster .......

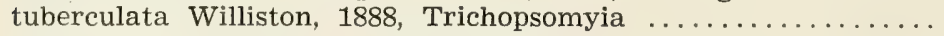

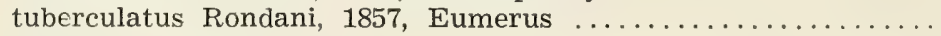

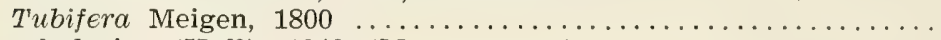
tubularius (Hull), 1942 (Mesogramma), Toxomerus .........

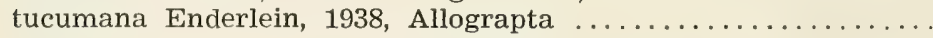
tumicephalum (Hull), 1943 (Volucella), Copestylum ......... tympanitis (Fabricius), 1805 (Syrphus), Copestylum .........

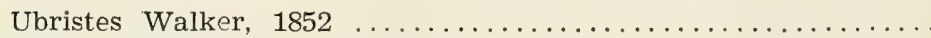

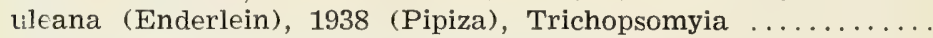

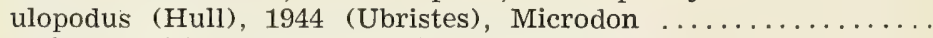

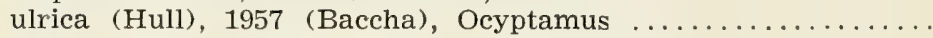

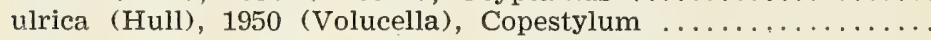
ultimus (Hull), 1951 (Mesogramma), Toxomerus . . . . . . . . .

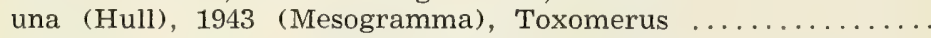

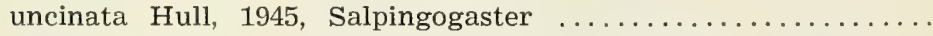
undecimpunctatus (Enderlein), 1938 (Mitrosphen), Toxomerus ..

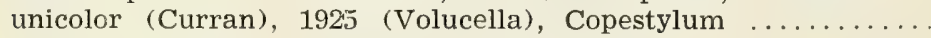

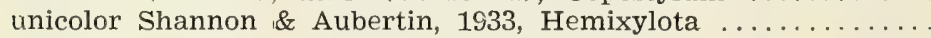

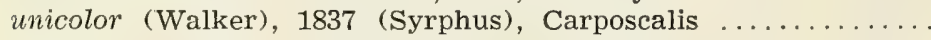

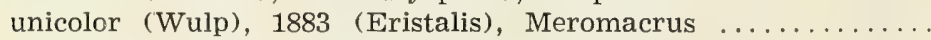
unilectum (Walker), 1860 (Temnocera), Copestylum ......... unimaculata Townsend, 1897, Nausigaster ............... unipunctatum (Curran), 1926 (Volucella), Copestylum .......

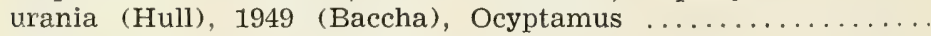

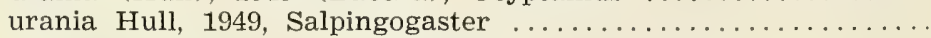

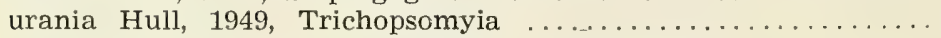

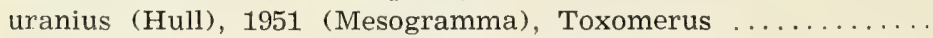

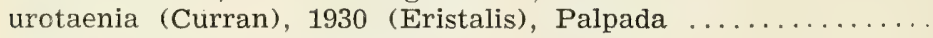

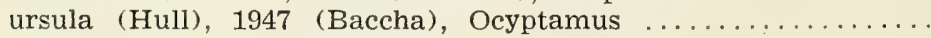

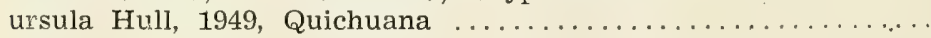
uvarum (Walker), 1849 (Eristalis), Palpada 


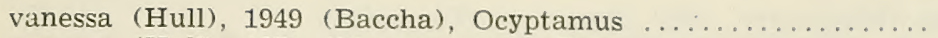

46.29

vanessa (Hull), 1951 (Mesogramma), Toxomerus ........... 46.56

vanzolinii d'Andretta \& Carrera, 1952, Nausigaster ......... 46.93

varia (Walker), 1849 (Baccha), Pseudodoros .............. 46.11

variabilis (Kertész), 1903 (Ceria), Sphiximorpha .............. 46.94

variabilis (Wulp), 1883 (Mesograpta), Toxomerus .......... 46.48

varians (Bigot), 1875 (Volucella), Copestylum ........... 46.86

varichaetum (Curran), 1925 (Phalacromyia), Copestylum ..... 46.86

variegatum (Bigot), 1875 (Volucella), Copestylum .......... 46.86

variegatus (Macquart), 1842 (Baccha), Ocyptamus ......... 46.29

varipes Shannon \& Aubertin, 1933, Hemixylota ............ 46.121

vatius (Walker), 1852 (Syrphus), Toxomerus ........... 46.56

ventana (Fluke), 1951 (Volucella), Copestylum ........... 46.86

venusta Curran, 1927, Allograpta .................... 46.38

vera (Hull), 1942 (Volucella), Copestylum ............... 46.86

vera (Hull), 1943 (Baccha), Ocyptamus ................ 46.29

vera Hull, 1944, Salpingogaster ................... 46.32

vera (Hull), 1949 (Eristalis), Palpada ............... 46.110

verdigaster (Hull), 1943 (Volucelia), Copestylum .......... 46.86

verona (Curran), 1941 (Baccha), Ocyptamus ............. 46.29

verralli (Williston), 1892 (Ceria), Monoceromyia ........... 46.94

vertebratus Rondani, 1863 (Syrphus) ................. 46.40

verticalis (Curran), 1927 (Mesogramma), Toxomerus ...... 46.56

verticallis, Hull, 1943, Toxomerus ................. 46.56

vesiculosum (Fabricius), 1805 (Syrphus), Copestylum ....... 46.80

vespuccius (Hull), 1943 (Baccha), Ocyptamus ............ 46.29

veve (Hull), 1942 (Mesogramma), Toxomerus ............ 46.56

vicina (Kertész), 1902 (Ceria), Sphiximorpha ............ 46.94

vicinum (Bigot), 1883 (Phalacromyia), Copestylum ......... 46.86

vicinus (Macquart), 1846 (Syrphus), Toxomerus ........... 46.56

victoria (Hull), 1942 (Baccha), Ocyptamus ............... 46.29

vierecki (Curran), 1930 (Baccha), Ocyptamus ............ 46.29

vierecki (Curran), 1930 (Eristalis), Palpada ............. 46.110

vierecki (Curran), 1930 (Mesogramma), Toxomerus ........ 46.56

vierecki (Curran), 1925 (Volucella), Copestylum ............ 46.86

Viereckomyia Curran, 1925 ......................... 46.70

villarica (Fluke), 1951 (Volucella), Copestylum ........... 46.86

villosa (Bigot), 1882 (Romaleosyrphus), Criorhina .......... 46.119

villosus Hull, 1949, Meromacrus .................. 46.113

vinetorum (Fabricius), 1798 (Syrphus), Palpada ............ 46.110

viola (Hull), 1944 (Volucella), Copestylum ............... 46.86

violacea (Macquart), 1842 (Volucella), Ornidia ............ 46.70

violaceum (Say), 1830 (Volucella), Copestylum ........... 46.79

violaceum (Curran), 1930 (Megametopon), Copestylum ...... 46.74

violaceus (Curran), 1926 (Mesogramma), Toxomerus ....... 46.56

violaceus (Hull), 1943 (Baccha), Ocyptamus ............. 46.29

violaceus (Macquart), 1842 (Aphritis), Microdon ........... 46.67

violens Townsend, 1895, Microdon .................. 46.67

virescens (Williston), 1891 (Phalacromyia), Copestylum ..... 46.86

virga (Fluke), 1942 (Epistrophe), Ocyptamus ............ 46.29

virgata Austen, 1893, Salpingogaster ................ 46.32

virgilio (Hull), 1942 (Baccha), Ocyptamus .............. 46.29

virginiensis (Drury), 1773 (Musca), Milesia ............... 46.120

virginio (Hull), 1941 (Baccha), Ocyptamus .............. 46.29

virgo Curran, 1940, Microdon .................... 46.67 
virgulatus (Macquart), 1850 (Syrphus), Toxomerus $\ldots \ldots \ldots \ldots$

46.56

viridana (Townsend), 1897 (Volucella), Copestylum ........ 46.86

viride (Williston), 1888 (Volucella), Copestylum .......... 46.87

viridigaster (Hull), 1943 (Volucella), Copestylum .......... 46.87

viridis Townsend, 1895, Microdon .................. 46.67

viridula (Bigot), 1875 (Volucella), Copestylum .......... 46.86

viridula (Walker), 1860 (Temnocera), Copestylum ......... 46.87

virtuosa (Hull), 1942 (Volucella), Copestylum ............ 46.87

vitrea (Huli), 1949 (Volucella), Copestylum ............... 46.87

viirescens (Hull), 1930 (Mesogramma), Toxomerus ......... 46.56

vitreus (Hull), 1941 (Mesogramma), Toxomerus ........... 46.56

vitripenne (Curran), 1934 (Volucella), Copestylum ......... 46.87

vitripennis Meigen, 1822, Syrphus .................. 46.8

vittata (Bigot), 1878 (Acrochordonodes), Senogaster ......... 46.120

vittata (Hull), 1937 (Meromacrus), Habromyia ........... 46.101

vittatum Thompson, Copestylum ................. 46.87

vittifacium (Hull), 1943 (Volucella), Copestylum ......... 46.87

vittiger (Hull), 1949 (Baccha), Ocyptamus ............... 46.30

vittipes (Curran), 1941 (Cerioides), Monoceromyia .......... 46.94

vittithorax (Hull), 1937 (Braziliana), Rhyops .............. 46.43

vockerothi Etcheverry, 1966, Flukea ................ 46.118

volatica (Williston), 1888 (Eristalis), Palpada ........... 46.107

volcanus (Fluke), 1942 (Epistrophe), Ocyptamus .......... 46.30

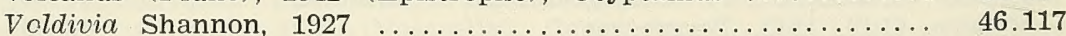

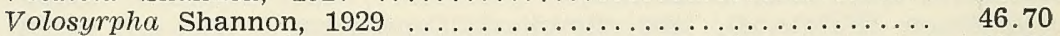

Volucellini .............................. 46.69

volucelloides (Bigot), 1884 (Glaurotricha), Copestylum ...... 46.87

Volucellosia Curran, 1930 ......................... 46.70

volucre (Giglio-Tos), 1892 (Volucella), Copestylum ......... 46.87

volucris Osten Sacken, 1877, Eupeodes ................. 46.9

vulcan (Hull), 1942 (Volucella), Copestylum ............... 46.87

vulpina Sack, 1941, Mallota ........................ 46.100

vulta (Fluke), 1951 (Volucella), Copestylum ........... 46.87

vilturus (Hull), 1942 (Mesogramma), Toxomerus ......... 46.56

vulturella (Hull), 1947 (Lepidostola), Lepidomyia .......... 46.89

walkeri (Lynch Arribálzaga), 1892 (Syrphus), Carposcalis . . . . 46.41

watsoni (Curran), 1930 (Mesogramma), Toxomerus ........ 46.56

watsoni (Curran), 1930 (Volucella), Copestylum .......... 46.82

wheeleri Mann, 1928, Microdon ...................... 46.62

wiedemanni Enderlein, 1938, Ocyptamus .................. 46.30

wiedemanni (Johnson), 1919 (Syrphus), Metasyrphus ........ 46.9

wilhelmina (Doesburg), 1963 (Baccha), Ocyptamus ......... 46.30

willinki (Fluke), 1951 (Volucella), Copestylum........... 46.87

willistoni Coquillett, 1902, Lycastrirhyncha ............... 46.102

willistoni Fluke, 1942, Syrphus .................... 46.8

willistoni (Giglio-Tos), 1893 (Sphaerophoria), Allograpta ...... 46.38

willistoni (Goot), 1964 (Heliophilus), Xylota .............. 46.114

willistoni Hull, 1949, Cacoceria ........................ 46.91

willistoni Mik, 1899, Microdon .................. 46.67

willistoni (Shannon), 1926 (Planes), Neplas .............. 46.115

willistoni (Snow), 1895 (Pelecocera), Chamaesyrphus ....... 46.69

willistoni Thompson, Ocyptamus .................. 46.30

willistoni (Townsend), 1895 (Eristalis), Palpada .............. 46.103

willistonii (Lynch Arribálzaga), 1892 (Mesogramma), Toxomerus 46.56 
Willistonimyia Hull, 1943

wulpianus (Lynch Arribálzaga), 1891 (Baccha), Ocyptamus ... 46.30

wulpi (Goot), 1964 (Volucella), Copestylum .............. 46.87

wulpii (Williston), 1888 (Ceria), Sphiximorpha .......... 46.94

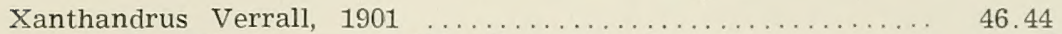

xanthaspis (Wiedemann), 1830 (Eristalis), Palpada .......... 46.105

xanthoprosopus (Barretto \& Lane), 1947 (Rhoga), Microdon ... 46.60

xanthopterus (Wiedemann), 1830 (Syrphus), Ocyptamus ..... 46.30

xantippe (Hull), 1949 (Baccha), Ocyptamus .............. 46.30

Xestoprosopa Hull, 1949 .......................... 46.46

xipe (Hull), 1942 (Volucella), Copestylum ............. 46.87

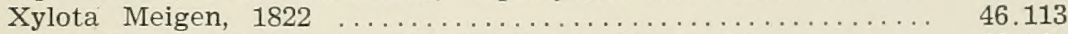

xylotaeformis (Schiner), 1868 (Mallota), Habromyia ......... 46.101

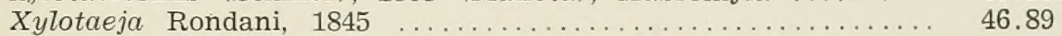

yura (Curran), 1930 (Volucella), Copestylum ........... 46.87

zabulon (Hull), 1949 (Baccha), Ocyptamus .............. 46.30

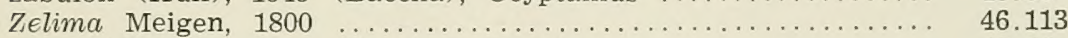

zenia (Curran), 1941 (Baccha), Ocyptamus ............. 46.30

zenilla (Hull), 1943 (Baccha), Leucopodella ............ 46.47

zenillia (Curran), 1941 (Baccha), Ocyptamus ............ 46.30

zephyra (Curran), 1939 (Volucella) Copestylum ............. 46.87

zephyreus (Hull), 1947 (Baccha), Ocyptamus ........... 46.30

zerene (Hull), 1949 (Baccha), Ocyptamus ............... 46.30

zeteki (Curran), 1930 (Baccha), Ocyptamus ............ 46.30

zilla (Hull), 1943 (Baccha), Ocyptamus ............... 46.30

zinnia (Hull), 1943 (Baccha), Ocyptamus .............. 46.30

zita (Curran), 1941 (Baccha), Ocyptamus .............. 46.30

zobeide (Hull), 1943 (Baccha), Ocyptamus .............. 46.31

zonatus (Loew), 1866 (Pteroptila), Meromacrus ........... 46.113

Zonemyia Shannon, $1925 \ldots \ldots \ldots \ldots \ldots \ldots \ldots \ldots \ldots \ldots \ldots \ldots . \ldots \ldots . \ldots \ldots$

zoroaster (Hull), 1943 (Baccha), Ocyptamus ............ 46.31 


$$
m g 1614879
$$

